\title{
Palladium-Catalyzed Highly Regioselective Aromatic Substitution of Benzylic Ammonium Salts with Amines
}

\author{
Ya-Nan Xu, ${ }^{\dagger}$ Meng-Zeng Zhu, ${ }^{\dagger}$ Yu-Kun Lin, ${ }^{\dagger}$ and Shi-Kai Tian*, ${ }^{\dagger}, \dagger$
}

${ }^{\dagger}$ Hefei National Laboratory for Physical Sciences at the Microscale, Center for Excellence in Molecular Synthesis, and Department of Chemistry, University of Science and Technology of China, Hefei, Anhui 230026, China

E-mail: tiansk@ustc.edu.cn

*Key Laboratory of Synthetic Chemistry of Natural Substances, Shanghai Institute of Organic Chemistry, Chinese Academy of Sciences, Shanghai 200032, China

\section{Supporting Information}

\section{Table of Contents}

General Information. .S2

Preparation of Benzylic Ammonium Salts and Amines......................................S2

General Procedure for the Aromatic Substitution of Benzylic Ammonium Salts with

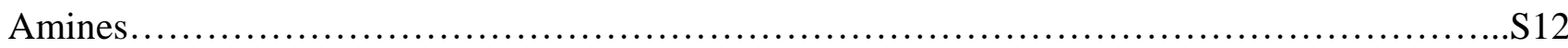

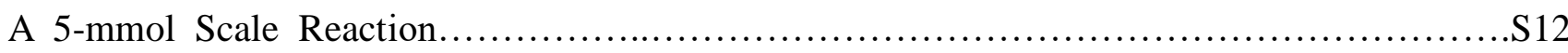

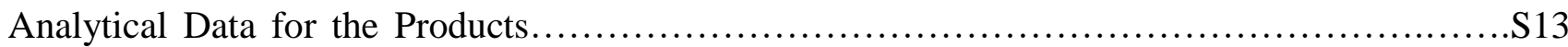

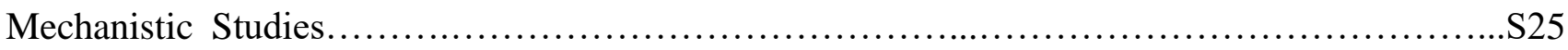

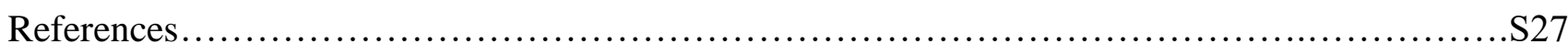

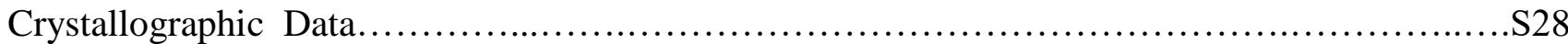

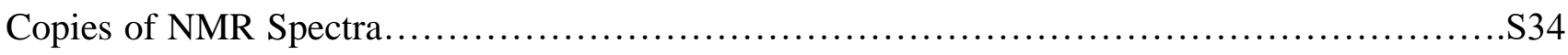




\section{General Information}

The ${ }^{1} \mathrm{H}$ NMR, ${ }^{13} \mathrm{C}$ NMR, ${ }^{19} \mathrm{~F}$ NMR, and ${ }^{31} \mathrm{P}$ NMR spectra were recorded on a Bruker AC-400 FT spectrometer (400 MHz, $100 \mathrm{MHz}, 376 \mathrm{MHz}$, and $162 \mathrm{MHz}$, respectively). The chemical shifts of ${ }^{1} \mathrm{H}$ $\mathrm{NMR}$ and ${ }^{13} \mathrm{C}$ NMR spectra were referenced internally with tetramethylsilane $(\delta \mathrm{H} 0.00, \delta \mathrm{C} 0.0)$, or residual protio solvent signals $\mathrm{CDCl}_{3}(\delta \mathrm{C} 77.2)$ or $\mathrm{CD}_{3} \mathrm{OD}(\delta \mathrm{H} 3.31, \delta \mathrm{C} 49.0)$. The chemical shifts of ${ }^{19} \mathrm{~F}$ NMR spectra were referenced to external trifluoroacetic acid. The chemical shifts of ${ }^{31} \mathrm{P}$ NMR spectra were referenced to external phosphoric acid. Chemical shifts $(\delta)$ and coupling constants $(J)$ were expressed in ppm and $\mathrm{Hz}$, respectively. The following abbreviations are used in reporting NMR data: s, singlet; d, doublet; t, triplet; q, quartet; m, multiplet; br, broad. High resolution mass spectra (HRMS) were recorded on a LC-TOF spectrometer (Micromass). ESI-mass data were acquired using a Thermo LTQ Orbitrap XL instrument equipped with an ESI source and controlled by Xcalibur software. Melting points are uncorrected.

Chemicals were purchased from the Energy Chemical, Acros, Accela, Alfa Aesar, and TCI, and used as received. Benzylic ammonium salts $\mathbf{1} \mathbf{a a}^{1}$ and $\mathbf{1 a b},{ }^{2}$ aldehydes $\mathbf{S 1},{ }^{3}$ and amines $\mathbf{S 3}^{4}$ were prepared according to literature procedures.

Abbreviations: $\mathrm{Ac}=$ acetyl, BINAP $=2,2^{\prime}$-bis(diphenylphosphino)-1,1'-binaphthyl, $\mathrm{COD}=$ 1,5-cyclooctadiene, $\mathrm{Cy}=$ cyclohexyl, $\mathrm{dba}=$ dibenzylideneacetone, $\mathrm{DBU}=1,8$-diazabicyclo [5,4,0]-7-undecene, dppb = 1,4-bis(diphenylphosphino)buthane, $\operatorname{dppf}=1,1^{\prime}$-bis(diphenylphosphino)ferrocene, $\mathrm{dppp}=1,3$-bis(diphenylphosphino)propane, $\mathrm{MTBE}=$ tert-butyl methyl ether, $\mathrm{Tf}=$ trifluoromethanesulfonyl, THF $=$ tetrahydrofuran.

\section{Preparation of Benzylic Ammonium Salts and Amines}

(1) Preparation of Ammonium Salts $\mathbf{1 a - \mathbf { h } ^ { 5 , 6 }}$

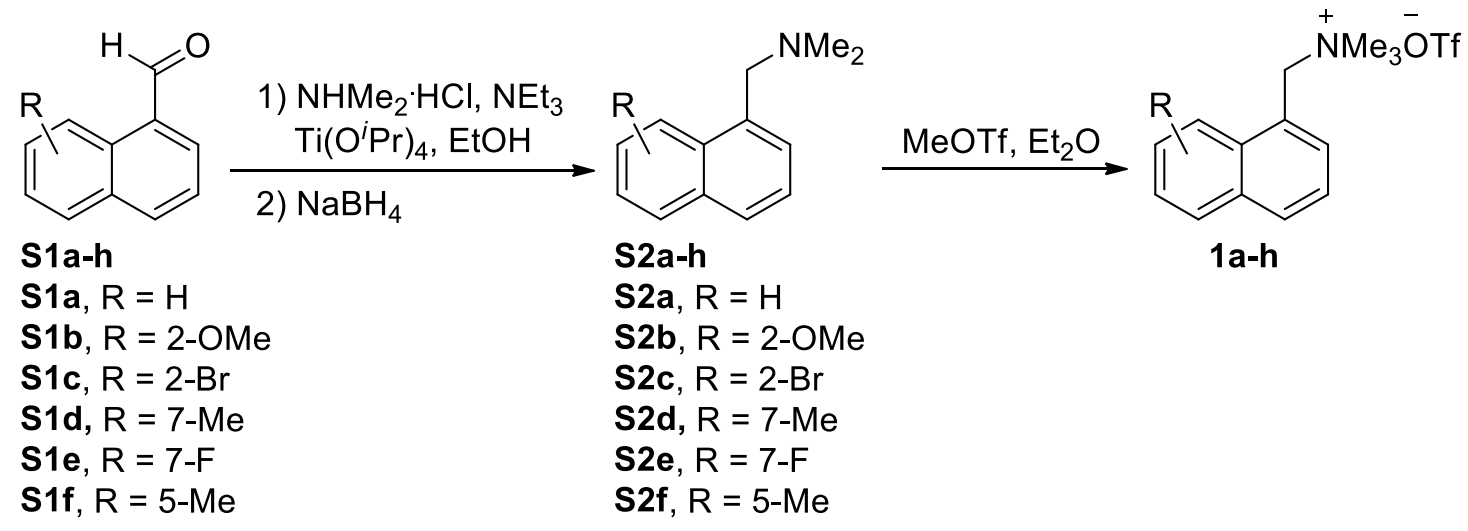<smiles>O=Cc1c2ccccc2cc2ccccc12</smiles>

S1g<smiles>O=Cc1cccc2c1ccc1ccccc12</smiles>

S1h<smiles>CN(C)Cc1c2ccccc2cc2ccccc12</smiles>

S2g<smiles>CN(C)Cc1cccc2c1ccc1ccccc12</smiles>

S2h

To a solution of triethylamine $(2.02 \mathrm{~g}, 2.78 \mathrm{~mL}, 20.0 \mathrm{mmol})$ in absolute ethanol $(15 \mathrm{~mL})$ were added dimethylamine hydrochloride $(1.65 \mathrm{~g}, 20.0 \mathrm{mmol})$, titanium(IV) isopropoxide (5.68 g, 5.92 $\mathrm{mL}, 20 \mathrm{mmol})$, and aldehyde $\mathbf{S 1 a}-\mathbf{h}(10.0 \mathrm{mmol})$. The mixture was stirred at room temperature for 10 $\mathrm{h}$, after which sodium borohydride $(567 \mathrm{mg}, 15.0 \mathrm{mmol})$ was added. The mixture was stirred at room 
temperature for $10 \mathrm{~h}$. The reaction was quenched by pouring the mixture into aqueous ammonia $(2 \mathrm{~N}$, $30 \mathrm{~mL}$ ), and the resulting inorganic precipitate was filtered and washed with dichloromethane (50 $\mathrm{mL})$. The aqueous filtrate was extracted with dichloromethane $(2 \times 50 \mathrm{~mL})$, and the combined dichloromethane extracts were dried over anhydrous sodium sulfate, and concentrated under reduced pressure to give tertiary amine $\mathbf{S 2 a - h}$

To a solution of the above tertiary amine $\mathbf{S 2 a - h}$ in diethyl ether $(10 \mathrm{~mL})$ at $0{ }^{\circ} \mathrm{C}$ was added dropwise MeOTf $(1.71 \mathrm{~g}, 1.20 \mathrm{~mL}, 10.4 \mathrm{mmol})$. The mixture was stirred at $0{ }^{\circ} \mathrm{C}$ for an additional 15 min. The precipitate was isolated by filtration and washed with diethyl ether $(2 \times 10 \mathrm{~mL})$. The resulting solid was dried under vacuum to give ammonium salt $\mathbf{1 a - h}$.

The signal peak of most of the $\mathrm{CF}_{3}$ quartet for the triflate group in ammonium salts are rather weak and unobvious, for most of them we could find the $\mathrm{CF}_{3}$ quartet being at $121 \mathrm{ppm}$ with $J=316$ $\mathrm{Hz}$ approximately. From some cases, 1e, 11, and 1m, we could not find or hardly distinguish the shape of quartet and therefore missed it out. ${ }^{6,7}$

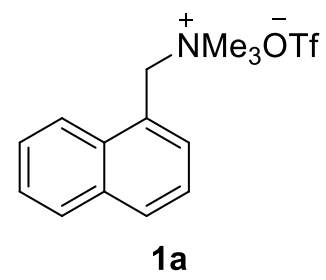

$N, N, N$-Trimethyl-1-(naphthalen-1-yl)methanaminium trifluoromethanesulfonate (1a), white solid (3.07 g, 88\% yield for two steps), m.p. 105-108 ${ }^{\circ} \mathrm{C} .{ }^{1} \mathrm{H} \mathrm{NMR}\left(400 \mathrm{MHz}, \mathrm{CD}_{3} \mathrm{OD}\right) \delta 8.35$ (d, $J=$ $8.0 \mathrm{~Hz}, 1 \mathrm{H}), 8.09(\mathrm{~d}, J=8.0 \mathrm{~Hz}, 1 \mathrm{H}), 7.99(\mathrm{~d}, J=7.6 \mathrm{~Hz}, 1 \mathrm{H}), 7.78(\mathrm{~d}, J=6.4 \mathrm{~Hz}, 1 \mathrm{H}), 7.72-7.58(\mathrm{~m}$, $3 \mathrm{H}), 5.06(\mathrm{~s}, 2 \mathrm{H}), 3.15(\mathrm{~s}, 9 \mathrm{H}) ;{ }^{13} \mathrm{C} \mathrm{NMR}\left(100 \mathrm{MHz}, \mathrm{CD}_{3} \mathrm{OD}\right) \delta 135.6,134.9,134.3,133.2,130.4$, 128.9, 127.6, 126.2, 125.1, 124.5, $121.8\left(\mathrm{q}, J_{C-F}=316.6 \mathrm{~Hz}\right), 66.4,53.7,53.6 ;{ }^{19} \mathrm{~F}$ NMR $(376 \mathrm{MHz}$, $\left.\mathrm{CD}_{3} \mathrm{OD}\right) \delta$-80.01; HRMS (ESI) calcd for $\mathrm{C}_{14} \mathrm{H}_{18} \mathrm{~N}^{+}(\mathrm{M}-\mathrm{OTf})^{+} 200.1434$, found 200.1428.<smiles></smiles>

1-(2-Methoxynaphthalen-1-yl)- $N, N, N$-trimethylmethanaminium trifluoromethanesulfonate (1b), brown solid (3.15 g, 83\% yield for two steps), m.p. 139-142 ${ }^{\circ} \mathrm{C} .{ }^{1} \mathrm{H}$ NMR (400 MHz, CD $\left.3 \mathrm{OD}\right) \delta 8.14$ $(\mathrm{d}, J=8.8 \mathrm{~Hz}, 1 \mathrm{H}), 8.08(\mathrm{~d}, J=9.2 \mathrm{~Hz}, 1 \mathrm{H}), 7.87(\mathrm{~d}, J=8.0 \mathrm{~Hz}, 1 \mathrm{H}), 7.65-7.59(\mathrm{~m}, 1 \mathrm{H}), 7.48(\mathrm{~d}, J=$ $9.2 \mathrm{~Hz}, 1 \mathrm{H}), 7.44-7.38(\mathrm{~m}, 1 \mathrm{H}), 4.98(\mathrm{~s}, 2 \mathrm{H}), 4.03(\mathrm{~s}, 3 \mathrm{H}), 3.24(\mathrm{~s}, 9 \mathrm{H}) ;{ }^{13} \mathrm{C} \mathrm{NMR}(100 \mathrm{MHz}$, $\left.\mathrm{CD}_{3} \mathrm{OD}\right) \delta 159.6,135.3,135.2,130.6,130.3,129.4,125.1,123.6,121.8\left(\mathrm{q}, J_{C-F}=316.6 \mathrm{~Hz}\right), 113.9$, 109.4, 60.5, 56.8, 54.0; ${ }^{19} \mathrm{~F}$ NMR (376 MHz, CD $\left.3 \mathrm{OD}\right) \delta-79.82$; HRMS (ESI) calcd for $\mathrm{C}_{15} \mathrm{H}_{20} \mathrm{ON}^{+}$ $(\mathrm{M}-\mathrm{OTf})^{+} 230.1539$, found 230.1541 . 


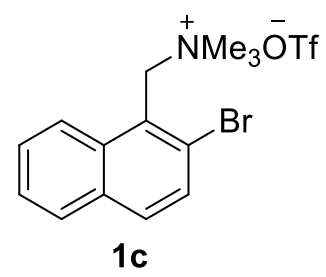

1-(2-Bromonaphthalen-1-yl)- $N, N, N$-trimethylmethanaminium trifluoromethanesulfonate (1c), yellow solid (3.43 g, 80\% yield for two steps), m.p. $157-160{ }^{\circ} \mathrm{C} .{ }^{1} \mathrm{H}$ NMR $\left(400 \mathrm{MHz}, \mathrm{CD}_{3} \mathrm{OD}\right) \delta$ $8.36(\mathrm{~d}, J=8.4 \mathrm{~Hz}, 1 \mathrm{H}), 8.02-7.97(\mathrm{~m}, 2 \mathrm{H}), 7.83(\mathrm{~d}, J=8.8 \mathrm{~Hz}, 1 \mathrm{H}), 7.77-7.71(\mathrm{~m}, 1 \mathrm{H}), 7.68-7.62$ $(\mathrm{m}, 1 \mathrm{H}), 5.47(\mathrm{~d}, J=14.4 \mathrm{~Hz}, 1 \mathrm{H}), 5.14(\mathrm{~d}, J=14.4 \mathrm{~Hz}, 1 \mathrm{H}), 3.24(\mathrm{~s}, 9 \mathrm{H}) ;{ }^{13} \mathrm{C}$ NMR $(100 \mathrm{MHz}$, $\left.\mathrm{CD}_{3} \mathrm{OD}\right) \delta 135.6,134.6,134.5,131.9,130.5,129.9,129.8,128.2,125.4,124.7,121.8\left(\mathrm{q}, J_{C-F}=\right.$ $305.3 \mathrm{~Hz}$ ), 65.2, 54.8; ${ }^{19} \mathrm{~F}$ NMR (376 MHz, CD $\left.{ }_{3} \mathrm{OD}\right) \delta-80.05$; HRMS (ESI) calcd for $\mathrm{C}_{14} \mathrm{H}_{17} \mathrm{BrN}^{+}$ (M - OTf) 278.0539 , found 278.0540.<smiles>Cc1ccc2cccc(CN(C)C)c2c1</smiles>

$\mathrm{N}, \mathrm{N}, \mathrm{N}$-Trimethyl-1-(7-methylnaphthalen-1-yl)methanaminium trifluoromethanesulfonate (1d), white solid (3.09 g, 85\% yield for two steps), m.p. $96-99{ }^{\circ} \mathrm{C} .{ }^{1} \mathrm{H} \mathrm{NMR}\left(400 \mathrm{MHz}, \mathrm{CDCl}_{3}\right) \delta 7.97$ (s, $1 \mathrm{H}), 7.81(\mathrm{~d}, J=8.4 \mathrm{~Hz}, 1 \mathrm{H}), 7.68(\mathrm{~d}, J=8.4 \mathrm{~Hz}, 1 \mathrm{H}), 7.60(\mathrm{~d}, J=6.8 \mathrm{~Hz}, 1 \mathrm{H}), 7.36-7.26(\mathrm{~m}, 2 \mathrm{H})$, 4.99 (s, $2 \mathrm{H}), 3.15$ (s, 9H), 2.51 (s, 3H); ${ }^{13} \mathrm{C}$ NMR $\left(100 \mathrm{MHz}, \mathrm{CDCl}_{3}\right) \delta 138.5,133.8,133.0,132.2$, $131.8,129.0,128.9,124.1,122.6,122.3,120.7\left(\mathrm{q}, J_{C-F}=316.6 \mathrm{~Hz}\right), 65.6,53.1,21.9 ;{ }^{19} \mathrm{~F}$ NMR $(376$ $\left.\mathrm{MHz}, \mathrm{CDCl}_{3}\right) \delta$-78.36; HRMS (ESI) calcd for $\mathrm{C}_{15} \mathrm{H}_{20} \mathrm{~N}^{+}(\mathrm{M}-\mathrm{OTf})^{+} 214.1590$, found 214.1590 .

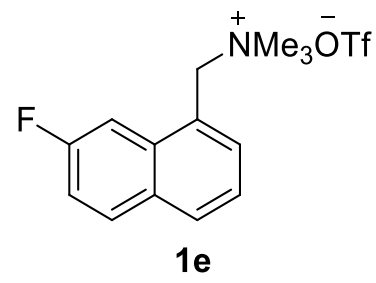

1-(7-Fluoronaphthalen-1-yl)- $N, N, N$-trimethylmethanaminium trifluoromethanesulfonate (1e), brown solid (2.79 g, 76\% yield for two steps), m.p. 78-81 ${ }^{\circ} \mathrm{C} .{ }^{1} \mathrm{H}$ NMR $\left(400 \mathrm{MHz}, \mathrm{CD}_{3} \mathrm{OD}\right) \delta$ 8.16-8.05 (m, 3H), $7.85(\mathrm{~d}, J=7.2 \mathrm{~Hz}, 1 \mathrm{H}), 7.62(\mathrm{t}, J=7.6 \mathrm{~Hz}, 1 \mathrm{H}), 7.47-7.40(\mathrm{~m}, 1 \mathrm{H}), 5.02(\mathrm{~s}, 2 \mathrm{H})$, $3.17(\mathrm{~s}, 9 \mathrm{H}) ;{ }^{13} \mathrm{C}$ NMR $\left(100 \mathrm{MHz}, \mathrm{CD}_{3} \mathrm{OD}\right) \delta 163.4\left(\mathrm{~d}, J_{\mathrm{C}-\mathrm{F}}=245.4 \mathrm{~Hz}\right), 136.0,135.5\left(\mathrm{~d}, J_{\mathrm{C}-\mathrm{F}}=9.0\right.$ $\mathrm{Hz}), 133.4\left(\mathrm{~d}, J_{\mathrm{C}-\mathrm{F}}=9.4 \mathrm{~Hz}\right), 133.2,132.8,125.7\left(\mathrm{~d}, J_{\mathrm{C}-\mathrm{F}}=2.5 \mathrm{~Hz}\right), 124.8,117.8\left(\mathrm{~d}, J_{\mathrm{C}-\mathrm{F}}=25.5 \mathrm{~Hz}\right)$, $108.4\left(\mathrm{~d}, J_{\mathrm{C}-\mathrm{F}}=22.9 \mathrm{~Hz}\right), 66.4,53.6,53.5 ;{ }^{19} \mathrm{~F} \mathrm{NMR}\left(376 \mathrm{MHz}, \mathrm{CD}_{3} \mathrm{OD}\right) \delta-80.09,-116.82$; HRMS (ESI) calcd for $\mathrm{C}_{14} \mathrm{H}_{17} \mathrm{NF}^{+}(\mathrm{M}-\mathrm{OTf})^{+} 218.1340$, found 218.1338 . 


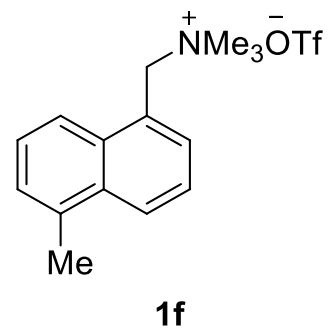

$N, N, N$-Trimethyl-1-(5-methylnaphthalen-1-yl)methanaminium trifluoromethanesulfonate (1f), white solid (3.05 g, 84\% yield for two steps), m.p. 141-144 ${ }^{\circ} \mathrm{C} .{ }^{1} \mathrm{H}$ NMR (400 MHz, $\left.\mathrm{CD}_{3} \mathrm{OD}\right) \delta 8.27$ $(\mathrm{d}, J=8.4 \mathrm{~Hz}, 1 \mathrm{H}), 8.20(\mathrm{~d}, J=8.4 \mathrm{~Hz}, 1 \mathrm{H}), 7.78(\mathrm{~d}, J=7.2 \mathrm{~Hz}, 1 \mathrm{H}), 7.68-7.53(\mathrm{~m}, 2 \mathrm{H}), 7.44(\mathrm{~d}, J=$ $6.8 \mathrm{~Hz}, 1 \mathrm{H}), 5.06(\mathrm{~s}, 2 \mathrm{H}), 3.15(\mathrm{~s}, 9 \mathrm{H}), 2.73(\mathrm{~s}, 3 \mathrm{H}) ;{ }^{13} \mathrm{C}$ NMR $\left(100 \mathrm{MHz}, \mathrm{CD}_{3} \mathrm{OD}\right) \delta$ 136.9, 134.6, 134.51, 134.47, 129.1, 128.5, 128.4, 126.0, 125.5, $122.8,121.8$ (q, $J_{C-F}=316.8 \mathrm{~Hz}$ ), 66.7, 53.6, 19.9; ${ }^{19} \mathrm{~F}$ NMR (376 MHz, CD $\left.{ }_{3} \mathrm{OD}\right) \delta-80.01$; HRMS (ESI) calcd for $\mathrm{C}_{15} \mathrm{H}_{20} \mathrm{~N}^{+}$(M - OTf) ${ }^{+} 214.1590$, found 214.1589 .

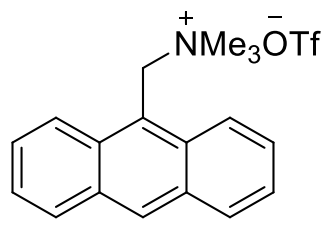

$1 \mathrm{~g}$

1-(Anthracen-9-yl)- $N, N, N$-trimethylmethanaminium trifluoromethanesulfonate (1g), white solid (3.28 g, 82\% yield for two steps), m.p. 205-208 ${ }^{\circ} \mathrm{C}$. ${ }^{1} \mathrm{H}$ NMR (400 MHz, CD $\left.{ }_{3} \mathrm{OD}\right) \delta$ 8.74-8.71 (m, $1 \mathrm{H}), 8.49(\mathrm{~d}, J=9.2 \mathrm{~Hz}, 2 \mathrm{H}), 8.13(\mathrm{~d}, J=8.4 \mathrm{~Hz}, 2 \mathrm{H}), 7.74-7.68(\mathrm{~m}, 2 \mathrm{H}), 7.59-7.54(\mathrm{~m}, 2 \mathrm{H}), 5.65(\mathrm{~s}$, 2H), $3.11(\mathrm{~s}, 9 \mathrm{H}) ;{ }^{13} \mathrm{C}$ NMR $\left(100 \mathrm{MHz}, \mathrm{CD}_{3} \mathrm{OD}\right) \delta 134.3,133.6,132.8,130.9,129.2,126.5,124.9$, $121.8\left(\mathrm{q}, J_{C-F}=316.6 \mathrm{~Hz}\right), 119.5,61.1,54.3 ;{ }^{19} \mathrm{~F}$ NMR $\left(376 \mathrm{MHz}, \mathrm{CD}_{3} \mathrm{OD}\right) \delta-80.03$; HRMS (ESI) calcd for $\mathrm{C}_{18} \mathrm{H}_{20} \mathrm{~N}^{+}$(M - OTf) ${ }^{+} 250.1590$, found 250.1590 .<smiles>C[N+](C)c1cccc2c(C[NH3+])cccc12</smiles>

$1 \mathrm{~h}$

$N, N, N$-Trimethyl-1-(phenanthren-1-yl)methanaminium trifluoromethanesulfonate (1h), white solid (3.20 g, 80\% yield for two steps), m.p. $92-95{ }^{\circ} \mathrm{C} .{ }^{1} \mathrm{H}$ NMR $\left(400 \mathrm{MHz}, \mathrm{CD}_{3} \mathrm{OD}\right) \delta 8.95(\mathrm{~d}, J=$ $8.4 \mathrm{~Hz}, 1 \mathrm{H}), 8.76(\mathrm{~d}, J=8.4 \mathrm{~Hz}, 1 \mathrm{H}), 8.21(\mathrm{~d}, J=9.2 \mathrm{~Hz}, 1 \mathrm{H}), 7.92(\mathrm{~d}, J=9.2 \mathrm{~Hz}, 2 \mathrm{H}), 7.84(\mathrm{~d}, J=$ $7.2 \mathrm{~Hz}, 1 \mathrm{H}), 7.77-7.61(\mathrm{~m}, 3 \mathrm{H}), 5.08(\mathrm{~s}, 2 \mathrm{H}), 3.15(\mathrm{~s}, 9 \mathrm{H}) ;{ }^{13} \mathrm{C}$ NMR (100 MHz, CD $\left.3 \mathrm{OD}\right) \delta 134.9$, 133.0, 132.8, 132.6, 131.5, 130.1, 129.6, 128.6, 128.5, 127.4, 127.1, 125.6, 124.1, 122.5, 121.8 (q, $\left.J_{\mathrm{C}-\mathrm{F}}=316.5 \mathrm{~Hz}\right), 66.5,53.5 ;{ }^{19} \mathrm{~F} \mathrm{NMR}\left(376 \mathrm{MHz}, \mathrm{CD}_{3} \mathrm{OD}\right) \delta-80.06$; HRMS (ESI) calcd for $\mathrm{C}_{18} \mathrm{H}_{20} \mathrm{~N}^{+}(\mathrm{M}-\mathrm{OTf})^{+} 250.1590$, found 250.1590 .

(2) Preparation of Ammonium Salts 1i-k $\mathbf{k}^{6,8}$ 


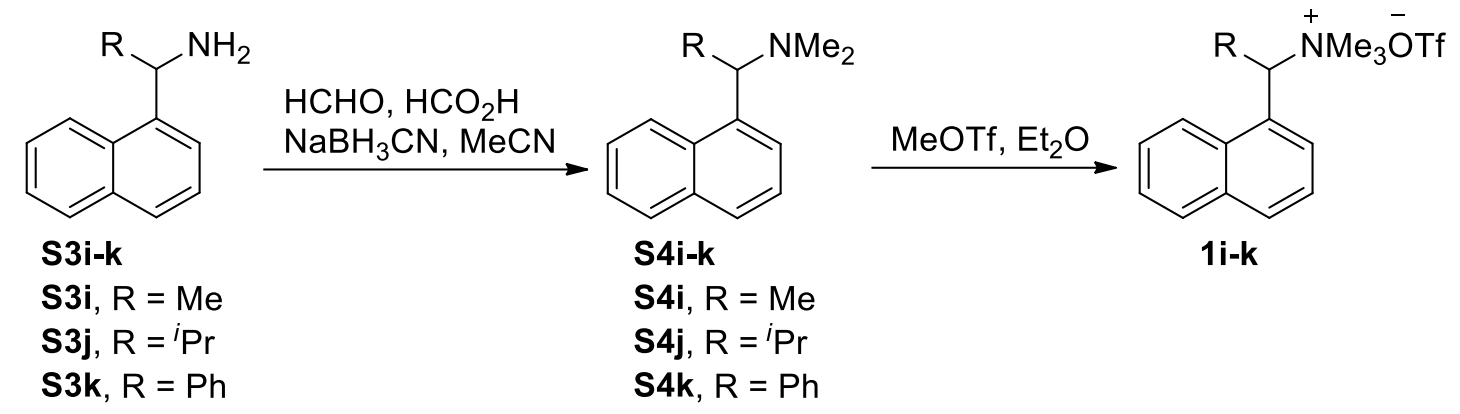

To formaldehyde (330 mg, $30 \%$ aqueous solution, $1.10 \mathrm{~mL}, 11.0 \mathrm{mmol})$ at $0{ }^{\circ} \mathrm{C}$ were added a solution of primary amine S3i-k $(5.0 \mathrm{mmol})$ in acetonitrile $(10 \mathrm{~mL})$, added portionwise $\mathrm{NaBH}_{3} \mathrm{CN}$ (628 mg, $10.0 \mathrm{mmol}$ ), and added dropwise formic acid $(1.15 \mathrm{~g}, 0.95 \mathrm{~mL}, 25.0 \mathrm{mmol})$. The mixture was stirred at room temperature for $5 \mathrm{~h}$, and quenched with aqueous ammonium chloride solution $(1.0 \mathrm{M}, 10 \mathrm{~mL})$. The organic layer was separated and the aqueous layer was extracted with ethyl acetate $(3 \times 10 \mathrm{~mL})$. The organic layers were combined, dried over anhydrous sodium sulfate, and concentrated under reduced pressure. The organic layer was added hydrochloric acid (1.0 M) until $\mathrm{pH}=2.0$. The aqueous layer was extracted with diethyl ether $(3 \times 10 \mathrm{~mL})$ and added aqueous sodium hydroxide $(1.0 \mathrm{M})$ until $\mathrm{pH}=13.0$. The mixture was extracted with diethyl ether $(3 \times 10 \mathrm{~mL})$, and the organic layer was washed with brine, dried over anhydrous sodium sulfate, and concentrated to give tertiary amine $\mathbf{S 4 i - k}$.

To a solution of the above tertiary amine S4i-k in diethyl ether $(5 \mathrm{~mL})$ at $0{ }^{\circ} \mathrm{C}$ was added dropwise MeOTf ( $853 \mathrm{mg}, 0.60 \mathrm{~mL}, 5.2 \mathrm{mmol})$. The mixture was stirred at $0{ }^{\circ} \mathrm{C}$ for $15 \mathrm{~min}$. The precipitate was isolated by filtration and washed with diethyl ether $(2 \times 10 \mathrm{~mL})$. The resulting solid was dried under vacuum to give ammonium salt 1i-k.<smiles>CC(C)c1cccc2ccccc12</smiles>

$N, N, N$-Trimethyl-1-(naphthalen-1-yl)ethanaminium trifluoromethanesulfonate (1i), colorless viscous oil $\left(1.31 \mathrm{~g}, 72 \%\right.$ yield for two steps). ${ }^{1} \mathrm{H}$ NMR $\left(400 \mathrm{MHz}, \mathrm{CDCl}_{3}\right) \delta 8.50(\mathrm{~d}, J=8.8 \mathrm{~Hz}, 1 \mathrm{H})$, $7.92(\mathrm{~d}, J=8.0 \mathrm{~Hz}, 1 \mathrm{H}), 7.85(\mathrm{~d}, J=8.0 \mathrm{~Hz}, 1 \mathrm{H}), 7.72(\mathrm{~d}, J=7.2 \mathrm{~Hz}, 1 \mathrm{H}), 7.64(\mathrm{t}, J=7.6 \mathrm{~Hz}, 1 \mathrm{H})$, 7.56-7.46 (m, 2H), $5.83(\mathrm{q}, J=6.4 \mathrm{~Hz}, 1 \mathrm{H}), 3.14(\mathrm{~s}, 9 \mathrm{H}), 1.88(\mathrm{~d}, J=6.4 \mathrm{~Hz}, 3 \mathrm{H}) ;{ }^{13} \mathrm{C}$ NMR $(100$ $\left.\mathrm{MHz}, \mathrm{CDCl}_{3}\right) \delta 133.8,132.3,131.5,129.2,128.7,128.1,127.9,126.5,124.9,122.9,120.7\left(\mathrm{q}, J_{\mathrm{C}-\mathrm{F}}=\right.$ $316.2 \mathrm{~Hz}$ ), 67.2, 51.1, 15.9; ${ }^{19} \mathrm{~F}$ NMR (376 MHz, $\left.\mathrm{CDCl}_{3}\right) \delta$-78.24; HRMS (ESI) calcd for $\mathrm{C}_{15} \mathrm{H}_{20} \mathrm{~N}^{+}$ $\left(\mathrm{M}\right.$ - OTf) ${ }^{+}$214.1590, found 214.1588. 
<smiles>CC(C)C(c1cccc2ccccc12)[N+](C)(C)C</smiles>

$1 \mathrm{j}$

$N, N, N, 2-T e t r a m e t h y l-1$ (naphthalen-1-yl)propan-1-aminium trifluoromethanesulfonate $\quad(\mathbf{1 j})$, colourless viscous oil (1.37 g, 70\% yield for two steps). ${ }^{1} \mathrm{H}$ NMR $\left(400 \mathrm{MHz}, \mathrm{CDCl}_{3}\right) \delta 8.48(\mathrm{~d}, J=$ $8.8 \mathrm{~Hz}, 1 \mathrm{H}), 7.97(\mathrm{~d}, J=8.0 \mathrm{~Hz}, 1 \mathrm{H}), 7.89(\mathrm{~d}, J=7.6 \mathrm{~Hz}, 1 \mathrm{H}), 7.77(\mathrm{~d}, J=7.2 \mathrm{~Hz}, 1 \mathrm{H}), 7.69(\mathrm{t}, J=$ $7.6 \mathrm{~Hz}, 1 \mathrm{H}), 7.61-7.51(\mathrm{~m}, 2 \mathrm{H}), 5.53(\mathrm{~d}, J=6.8 \mathrm{~Hz}, 1 \mathrm{H}), 3.24(\mathrm{~s}, 9 \mathrm{H}), 3.02-2.92(\mathrm{~m}, 1 \mathrm{H}), 1.16(\mathrm{~d}, J$ $=6.8 \mathrm{~Hz}, 3 \mathrm{H}), 1.03(\mathrm{~d}, J=6.8 \mathrm{~Hz}, 3 \mathrm{H}) ;{ }^{13} \mathrm{C} \mathrm{NMR}\left(100 \mathrm{MHz}, \mathrm{CDCl}_{3}\right) \delta 134.0,133.4,131.3,129.4$, $128.4,127.8,127.0,126.6,124.7,122.9,120.7\left(\mathrm{q}, J_{\mathrm{C}-\mathrm{F}}=318.2 \mathrm{~Hz}\right), 76.4,53.1,30.2,22.6,20.6 ;{ }^{19} \mathrm{~F}$ NMR (376 MHz, $\left.\mathrm{CDCl}_{3}\right) \delta-78.27$; HRMS (ESI) calcd for $\mathrm{C}_{17} \mathrm{H}_{24} \mathrm{~N}^{+}(\mathrm{M}-\mathrm{OTf})^{+} 242.1903$, found 242.1903 .

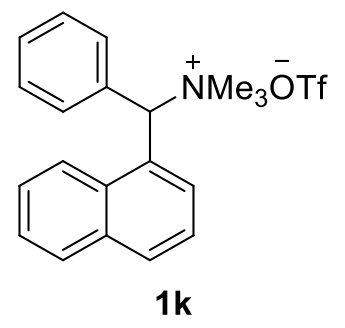

$N, N, N$-Trimethyl-1-(naphthalen-1-yl)-1-phenylmethanaminium trifluoromethanesulfonate (1k), white solid (1.32 g, 62\% yield for two steps), m.p. 128-131 ${ }^{\circ} \mathrm{C} .{ }^{1} \mathrm{H}$ NMR (400 MHz, $\left.\mathrm{CDCl}_{3}\right) \delta 8.74$ $(\mathrm{d}, J=8.4 \mathrm{~Hz}, 1 \mathrm{H}), 8.19(\mathrm{~d}, J=6.4 \mathrm{~Hz}, 1 \mathrm{H}), 7.93(\mathrm{~d}, J=6.8 \mathrm{~Hz}, 2 \mathrm{H}), 7.86(\mathrm{~d}, J=8.0 \mathrm{~Hz}, 1 \mathrm{H}), 7.80$ $(\mathrm{d}, J=8.4 \mathrm{~Hz}, 1 \mathrm{H}), 7.70(\mathrm{t}, J=7.2 \mathrm{~Hz}, 1 \mathrm{H}), 7.57(\mathrm{t}, J=7.2 \mathrm{~Hz}, 1 \mathrm{H}), 7.46(\mathrm{t}, J=7.2 \mathrm{~Hz}, 1 \mathrm{H})$, 7.38-7.28 (m, 3H), $6.77(\mathrm{~s}, 1 \mathrm{H}), 3.23(\mathrm{~s}, 9 \mathrm{H}) ;{ }^{13} \mathrm{C} \mathrm{NMR}\left(100 \mathrm{MHz}, \mathrm{CDCl}_{3}\right) \delta 134.1,132.3,132.2$, $131.3,131.0,130.4,129.7,129.4,128.5,128.1,128.0,126.7,125.0,122.8,120.9\left(\mathrm{q}, J_{\mathrm{C}-\mathrm{F}}=318.0 \mathrm{~Hz}\right)$, 75.5, 52.5; ${ }^{19} \mathrm{~F}$ NMR (376 MHz, $\left.\mathrm{CDCl}_{3}\right) \delta$-78.29; HRMS (ESI) calcd for $\mathrm{C}_{20} \mathrm{H}_{22} \mathrm{~N}^{+}$(M - OTf) 276.1747 , found 276.1747 .

(3) Preparation of Ammonium Salts 11-q $\mathbf{q}^{6,8}$ 


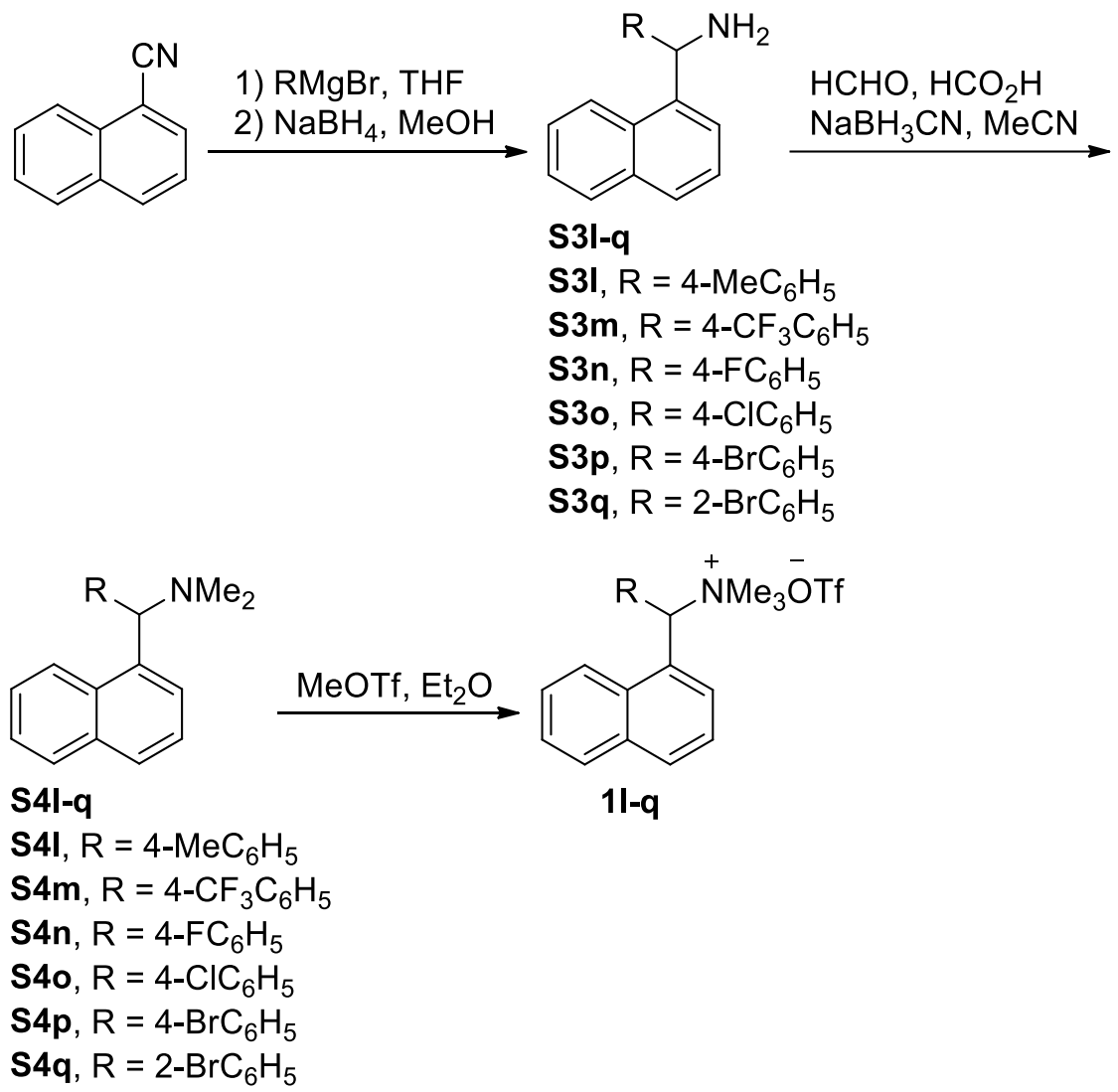

The Grignard reagent, prepared from the corresponding aryl bromide $(8.0 \mathrm{mmol})$ and magnesium powder $(413 \mathrm{mg}, 17.0 \mathrm{mmol})$ in tetrahydrofuran $(15 \mathrm{~mL})$, was added dropwise to a solution of 1-naphthonitrile $(766 \mathrm{mg}, 5.0 \mathrm{mmol})$ in tetrahydrofuran $(10 \mathrm{~mL})$ at $0{ }^{\circ} \mathrm{C}$ under nitrogen atmosphere. The mixture was stirred at $70{ }^{\circ} \mathrm{C}$ for $3 \mathrm{~h}$, and cooled to room temperature. The solvent was removed by evaporation, and the residue was dissolved in methanol $(10 \mathrm{~mL})$ at $0{ }^{\circ} \mathrm{C}$. Sodium borohydride (189 mg, $5.0 \mathrm{mmol}$ ) was added slowly and the mixture was stirred at $25^{\circ} \mathrm{C}$ for $16 \mathrm{~h}$. The mixture was concentrated under reduced pressure, and added hydrochloric acid $(1.0 \mathrm{M})$ until $\mathrm{pH}=$ 2.0. The aqueous layer was extracted with diethyl ether $(3 \times 10 \mathrm{~mL})$ and added aqueous sodium hydroxide $(1.0 \mathrm{M})$ until $\mathrm{pH}=13.0$. The mixture was extracted with diethyl ether $(3 \times 10 \mathrm{~mL})$, and the organic layer was washed with brine, dried over anhydrous sodium sulfate, and concentrated under reduced pressure to give primary amine S3I-q.

To formaldehyde (330 mg, 30\% aqueous solution, $1.10 \mathrm{~mL}, 11.0 \mathrm{mmol}$ ) at $0{ }^{\circ} \mathrm{C}$ were added a solution of the above primary amine S3I-q in acetonitrile $(10 \mathrm{~mL})$, added portionwise $\mathrm{NaBH}_{3} \mathrm{CN}$ (628 $\mathrm{mg}, 10.0 \mathrm{mmol})$, and added dropwise formic acid $(1.15 \mathrm{~g}, 0.95 \mathrm{~mL}, 25.0 \mathrm{mmol})$. The mixture was stirred at room temperature for $5 \mathrm{~h}$, and quenched with aqueous ammonium chloride solution $(1.0 \mathrm{M}, 10 \mathrm{~mL})$. The organic layer was separated and the aqueous layer was extracted with ethyl acetate $(3 \times 10 \mathrm{~mL})$. The organic layers were combined, dried over anhydrous sodium sulfate, and concentrated under reduced pressure. The organic layer was added hydrochloric acid (1.0 M) until $\mathrm{pH}=2.0$. The aqueous layer was extracted with diethyl ether $(3 \times 10 \mathrm{~mL})$ and added aqueous sodium hydroxide $(1.0 \mathrm{M})$ until $\mathrm{pH}=13.0$. The mixture was extracted with diethyl ether $(3 \times 10 \mathrm{~mL})$, and the organic layer was washed with brine, dried over anhydrous sodium sulfate, and concentrated to give tertiary amine $\mathbf{S 4 l - q}$.

To a solution of tertiary amine $\mathbf{S 4 I - q}$ in diethyl ether $(5 \mathrm{~mL})$ at $0{ }^{\circ} \mathrm{C}$ was added dropwise MeOTf 
(853 mg, $0.60 \mathrm{~mL}, 5.2 \mathrm{mmol}$ ). The mixture was stirred at $0{ }^{\circ} \mathrm{C}$ for $15 \mathrm{~min}$. The precipitate was isolated by filtration and washed with diethyl ether $(2 \times 10 \mathrm{~mL})$. The resulting solid was dried under vacuum to give benzylic ammonium salt $\mathbf{1 l}-\mathbf{q}$.

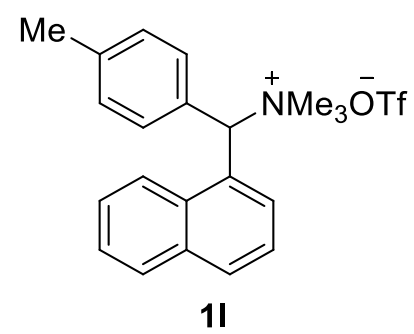

$N, N, N$-Trimethyl-1-(naphthalen-1-yl)-1-( $p$-tolyl)methanaminium trifluoromethanesulfonate (11), white solid (923 mg, 42\% yield for three steps), m.p. $130-133{ }^{\circ} \mathrm{C} .{ }^{1} \mathrm{H}$ NMR $\left(400 \mathrm{MHz}, \mathrm{CDCl}_{3}\right) \delta$ $9.21(\mathrm{~d}, J=8.8 \mathrm{~Hz}, 1 \mathrm{H}), 8.18(\mathrm{~d}, J=7.2 \mathrm{~Hz}, 1 \mathrm{H}), 7.98-7.91(\mathrm{~m}, 3 \mathrm{H}), 7.85(\mathrm{~d}, J=8.0 \mathrm{~Hz}, 1 \mathrm{H})$, 7.80-7.74 (m, 1H), $7.60(\mathrm{t}, J=8.0 \mathrm{~Hz}, 1 \mathrm{H}), 7.54(\mathrm{t}, J=7.2 \mathrm{~Hz}, 1 \mathrm{H}), 7.24(\mathrm{~d}, J=8.0 \mathrm{~Hz}, 2 \mathrm{H}), 7.05$ (s, $1 \mathrm{H}), 3.56(\mathrm{~s}, 9 \mathrm{H}), 2.30(\mathrm{~s}, 3 \mathrm{H}) ;{ }^{13} \mathrm{C} \mathrm{NMR}\left(100 \mathrm{MHz}, \mathrm{CDCl}_{3}\right) \delta 140.9,134.3,132.4,131.4,131.2$, $130.5,129.3,128.7,128.4,127.9,126.9,124.8,124.3,75.7,53.0,21.2 ;{ }^{19} \mathrm{~F} \mathrm{NMR}\left(376 \mathrm{MHz}, \mathrm{CDCl}_{3}\right)$ $\delta$-78.22; HRMS (ESI) calcd for $\mathrm{C}_{21} \mathrm{H}_{24} \mathrm{~N}^{+}$(M - OTf) 290.1903 , found 290.1903.

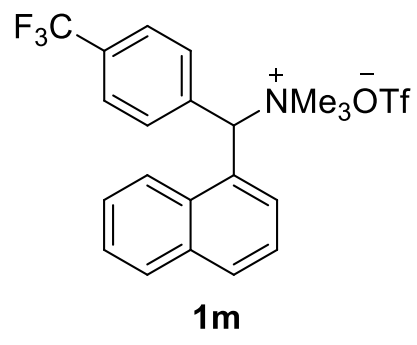

$N, N, N$-Trimethyl-1-(naphthalen-1-yl)-1-(4-(trifluoromethyl)phenyl)methanaminium trifluoromethanesulfonate $(\mathbf{1 m})$, brown viscous oil (1.14 g, 46\% yield for three steps). ${ }^{1} \mathrm{H}$ NMR (400 $\left.\mathrm{MHz}, \mathrm{CD}_{3} \mathrm{OD}\right) \delta 8.84(\mathrm{~d}, J=8.8 \mathrm{~Hz}, 1 \mathrm{H}), 8.41(\mathrm{~d}, J=7.2 \mathrm{~Hz}, 1 \mathrm{H}), 8.31(\mathrm{~d}, J=8.4 \mathrm{~Hz}, 2 \mathrm{H}), 8.06(\mathrm{~d}$, $J=8.0 \mathrm{~Hz}, 1 \mathrm{H}), 7.97(\mathrm{~d}, J=8.4 \mathrm{~Hz}, 1 \mathrm{H}), 7.82(\mathrm{~d}, J=8.4 \mathrm{~Hz}, 2 \mathrm{H}), 7.79-7.39(\mathrm{~m}, 1 \mathrm{H}), 7.70(\mathrm{t}, J=8.0$ $\mathrm{Hz}, 1 \mathrm{H}), 7.59(\mathrm{t}, J=7.2 \mathrm{~Hz}, 1 \mathrm{H}), 7.00(\mathrm{~s}, 1 \mathrm{H}), 3.37(\mathrm{~s}, 9 \mathrm{H}) ;{ }^{13} \mathrm{C}$ NMR $\left(100 \mathrm{MHz}, \mathrm{CD}_{3} \mathrm{OD}\right) \delta 138.6$, $135.8,133.6,133.1\left(\mathrm{q}, J_{\mathrm{C}-\mathrm{F}}=32.6 \mathrm{~Hz}\right), 132.8,132.7,130.8,130.3,129.4,128.8,127.7,127.5\left(\mathrm{q}, J_{\mathrm{C}-\mathrm{F}}\right.$ $=3.6 \mathrm{~Hz}), 126.4,126.3,123.7,75.9,53.7 ;{ }^{19} \mathrm{~F} \mathrm{NMR}\left(376 \mathrm{MHz}, \mathrm{CD}_{3} \mathrm{OD}\right) \delta-64.36,-79.71$; HRMS (ESI) calcd for $\mathrm{C}_{21} \mathrm{H}_{21} \mathrm{NF}_{3}{ }^{+}(\mathrm{M}-\mathrm{OTf})^{+} 344.1621$, found 344.1621 .

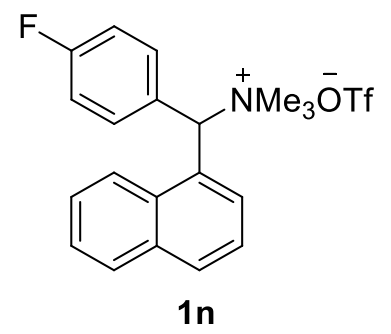

1-(4-Fluorophenyl)- $N, N, N$-trimethyl-1-(naphthalen-1-yl)methanaminium trifluoromethanesulfonate (1n), white solid (909 mg, 41\% yield for three steps), m.p. $110-113{ }^{\circ} \mathrm{C} .{ }^{1} \mathrm{H}$ 
NMR $\left(400 \mathrm{MHz}, \mathrm{CDCl}_{3}\right) \delta 8.73(\mathrm{~d}, J=8.8 \mathrm{~Hz}, 1 \mathrm{H}), 8.20(\mathrm{~d}, J=7.2 \mathrm{~Hz}, 1 \mathrm{H}), 8.01(\mathrm{dd}, J=8.8,4.8$ $\mathrm{Hz}, 2 \mathrm{H}), 7.90(\mathrm{~d}, J=8.4 \mathrm{~Hz}, 1 \mathrm{H}), 7.83(\mathrm{~d}, J=7.6 \mathrm{~Hz}, 1 \mathrm{H}), 7.73-7.67(\mathrm{~m}, 1 \mathrm{H}), 7.59$ (t, $J=7.6 \mathrm{~Hz}$, $1 \mathrm{H}), 7.49$ (t, $J=7.6 \mathrm{~Hz}, 1 \mathrm{H}), 7.09(\mathrm{t}, J=8.4 \mathrm{~Hz}, 2 \mathrm{H}), 6.83(\mathrm{~s}, 1 \mathrm{H}), 3.27(\mathrm{~s}, 9 \mathrm{H}) ;{ }^{13} \mathrm{C} \mathrm{NMR}(100 \mathrm{MHz}$, $\left.\mathrm{CDCl}_{3}\right) \delta 163.6\left(\mathrm{~d}, J_{C-\mathrm{F}}=250.7 \mathrm{~Hz}\right), 134.2,133.4\left(\mathrm{~d}, J_{C-\mathrm{F}}=8.4 \mathrm{~Hz}\right), 132.3,131.5,129.5,128.7$, 128.3, 128.0, 126.8, 125.0, 122.8, $120.9\left(\mathrm{q}, J_{C-\mathrm{F}}=318.1 \mathrm{~Hz}\right), 116.8\left(\mathrm{~d}, J_{C-\mathrm{F}}=21.5 \mathrm{~Hz}\right), 74.8,52.5$; ${ }^{19} \mathrm{~F}$ NMR (376 MHz, $\left.\mathrm{CDCl}_{3}\right) \delta-78.20,-109.41$; HRMS (ESI) calcd for $\mathrm{C}_{20} \mathrm{H}_{21} \mathrm{NF}^{+}(\mathrm{M}-\mathrm{OTf})^{+}$ 294.1653, found 294.1654.

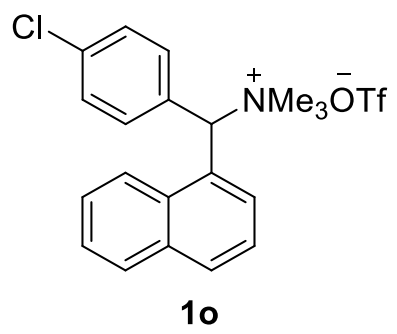

1-(4-Chlorophenyl)- $N, N, N$-trimethyl-1-(naphthalen-1-yl)methanaminium trifluoromethanesulfonate (1o), white solid (1.03 g, 45\% yield for three steps), m.p. 56-59 ${ }^{\circ} \mathrm{C} .{ }^{1} \mathrm{H}$ NMR $\left(400 \mathrm{MHz}, \mathrm{CDCl}_{3}\right) \delta 8.74(\mathrm{~d}, J=8.8 \mathrm{~Hz}, 1 \mathrm{H}), 8.19(\mathrm{~d}, J=7.2 \mathrm{~Hz}, 1 \mathrm{H}), 7.95(\mathrm{~d}, J=8.4 \mathrm{~Hz}$, 2H), $7.90(\mathrm{~d}, J=8.0 \mathrm{~Hz}, 1 \mathrm{H}), 7.83(\mathrm{~d}, J=7.6 \mathrm{~Hz}, 1 \mathrm{H}), 7.74-7.68(\mathrm{~m}, 1 \mathrm{H}), 7.59(\mathrm{t}, J=8.0 \mathrm{~Hz}, 1 \mathrm{H})$, $7.49(\mathrm{t}, J=7.6 \mathrm{~Hz}, 1 \mathrm{H}), 7.36(\mathrm{~d}, J=8.4 \mathrm{~Hz}, 2 \mathrm{H}), 6.83(\mathrm{~s}, 1 \mathrm{H}), 3.28(\mathrm{~s}, 9 \mathrm{H}) ;{ }^{13} \mathrm{C}$ NMR $(100 \mathrm{MHz}$, $\left.\mathrm{CDCl}_{3}\right) \delta 136.7,134.2,132.4,132.3,131.5,130.9,129.9,129.5,128.6,128.1,127.6,126.8,125.0$, $122.7,120.9\left(\mathrm{q}, J_{\mathrm{C}-\mathrm{F}}=318.2 \mathrm{~Hz}\right), 74.7,52.5 ;{ }^{19} \mathrm{~F} \mathrm{NMR}\left(376 \mathrm{MHz}, \mathrm{CDCl}_{3}\right) \delta-78.31 ; \mathrm{HRMS}(\mathrm{ESI})$ calcd for $\mathrm{C}_{20} \mathrm{H}_{21} \mathrm{NCl}^{+}(\mathrm{M}-\mathrm{OTf})^{+} 310.1357$, found 310.1357 .

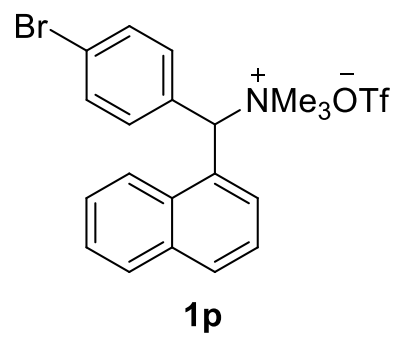

1-(4-Bromophenyl)- $N, N, N$-trimethyl-1-(naphthalen-1-yl)methanaminium

trifluoromethanesulfonate $(\mathbf{1 p})$, white solid $\left(1.01 \mathrm{~g}, 40 \%\right.$ yield for three steps), m.p. $69-72{ }^{\circ} \mathrm{C} .{ }^{1} \mathrm{H}$ NMR $\left(400 \mathrm{MHz}, \mathrm{CDCl}_{3}\right) \delta 8.70(\mathrm{~d}, J=8.8 \mathrm{~Hz}, 1 \mathrm{H}), 8.12(\mathrm{~d}, J=7.2 \mathrm{~Hz}, 1 \mathrm{H}), 7.93(\mathrm{~d}, J=8.0 \mathrm{~Hz}$, $1 \mathrm{H}), 7.88-7.83(\mathrm{~m}, 3 \mathrm{H}), 7.77-7.71(\mathrm{~m}, 1 \mathrm{H}), 7.62-7.51(\mathrm{~m}, 4 \mathrm{H}), 6.81(\mathrm{~s}, 1 \mathrm{H}), 3.28(\mathrm{~s}, 9 \mathrm{H}) ;{ }^{13} \mathrm{C} \mathrm{NMR}$ $\left(100 \mathrm{MHz}, \mathrm{CDCl}_{3}\right) \delta 134.3,133.1,132.6,132.4,131.7,131.4,129.6,128.9,128.0,127.7,127.0$, $125.3,124.9,122.8,120.9\left(\mathrm{q}, J_{\mathrm{C}-\mathrm{F}}=318.0 \mathrm{~Hz}\right), 74.9,52.6 ;{ }^{19} \mathrm{~F}$ NMR $\left(376 \mathrm{MHz}, \mathrm{CDCl}_{3}\right) \delta-80.08$; HRMS (ESI) calcd for $\mathrm{C}_{20} \mathrm{H}_{21} \mathrm{NBr}^{+}(\mathrm{M}-\mathrm{OTf})^{+} 354.0852$, found 354.0855 . 


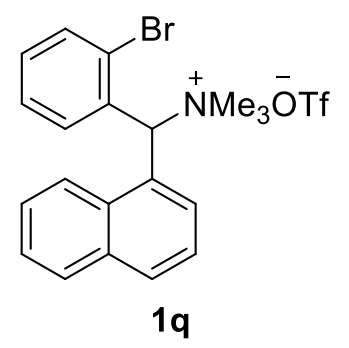

1-(2-Bromophenyl)- $N, N, N$-trimethyl-1-(naphthalen-1-yl)methanaminium

trifluoromethanesulfonate $(\mathbf{1 q})$, white solid $\left(1.08 \mathrm{~g}, 43 \%\right.$ yield for three steps), m.p. $78-81{ }^{\circ} \mathrm{C} .{ }^{1} \mathrm{H}$ NMR $\left(400 \mathrm{MHz}, \mathrm{CDCl}_{3}\right) \delta 8.53(\mathrm{~d}, J=8.8 \mathrm{~Hz}, 1 \mathrm{H}), 8.38(\mathrm{dd}, J=8.4,1.6 \mathrm{~Hz}, 1 \mathrm{H}), 8.32(\mathrm{~d}, J=7.2$ $\mathrm{Hz}, 1 \mathrm{H}), 7.97(\mathrm{~d}, J=8.4 \mathrm{~Hz}, 1 \mathrm{H}), 7.91(\mathrm{~d}, J=7.6 \mathrm{~Hz}, 1 \mathrm{H}), 7.76-7.70(\mathrm{~m}, 1 \mathrm{H}), 7.70-7.65(\mathrm{~m}, 2 \mathrm{H})$, 7.62-7.54 (m, 2H), 7.33-7.26 (m, 1H), $7.23(\mathrm{~s}, 1 \mathrm{H}), 3.36(\mathrm{~s}, 9 \mathrm{H}) ;{ }^{13} \mathrm{C} \mathrm{NMR}\left(100 \mathrm{MHz}, \mathrm{CDCl}_{3}\right) \delta$ $134.9,134.4,132.5,132.1,132.0,131.7,131.3,130.0,129.5,129.4,128.6,127.4,126.9,126.8$, $125.7,122.6,120.9\left(\mathrm{q}, J_{\mathrm{C}-\mathrm{F}}=316.6 \mathrm{~Hz}\right), 73.7,53.1 ;{ }^{19} \mathrm{~F}$ NMR $\left(376 \mathrm{MHz}, \mathrm{CDCl}_{3}\right) \delta-80.05 ; \mathrm{HRMS}$ (ESI) calcd for $\mathrm{C}_{20} \mathrm{H}_{21} \mathrm{NBr}^{+}(\mathrm{M}-\mathrm{OTf})^{+} 354.0852$, found 354.0854 .

(4) Preparation of Amine $\mathbf{S 4 r}$

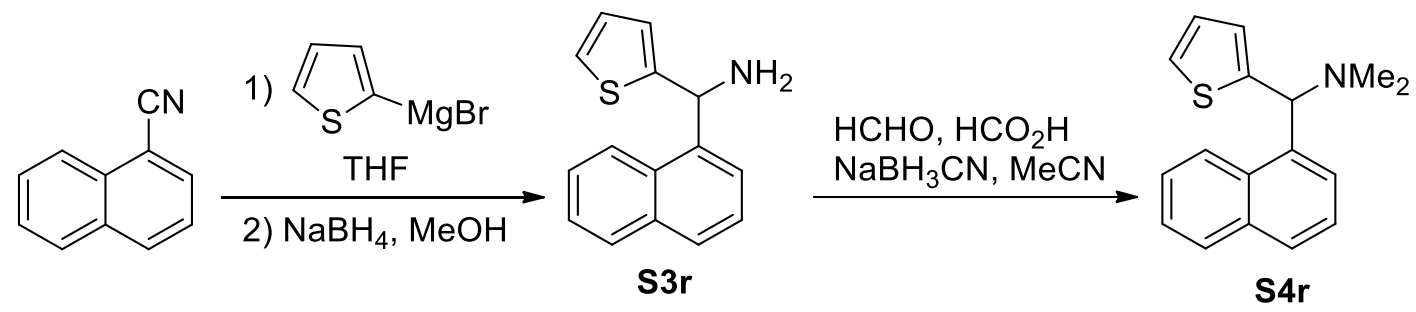

The Grignard reagent, prepared from 2-bromothiophene (2.44 g, $15.0 \mathrm{mmol})$ and magnesium powder $(413 \mathrm{mg}, 17.0 \mathrm{mmol})$ in tetrahydrofuran $(30 \mathrm{~mL})$ was added dropwise to a solution of 1-naphthonitrile $(1.53 \mathrm{~g}, 10 \mathrm{mmol})$ in tetrahydrofuran $(10 \mathrm{~mL})$ at $0{ }^{\circ} \mathrm{C}$ under nitrogen atmosphere. The resulting mixture was stirred at $70{ }^{\circ} \mathrm{C}$ for $3 \mathrm{~h}$, and cooled to room temperature. The solvent was removed by evaporation, and the residue dissolved in methanol $(10 \mathrm{~mL})$ at $0{ }^{\circ} \mathrm{C}$. Sodium borohydride (378 mg, $10 \mathrm{mmol}$ ) was added slowly and the mixture was stirred at $25{ }^{\circ} \mathrm{C}$ for $16 \mathrm{~h}$. The mixture was concentrated under reduced pressure, and added hydrochloric acid $(1.0 \mathrm{M})$ until $\mathrm{pH}=$ 2.0. The aqueous layer was extracted with diethyl ether $(3 \times 10 \mathrm{~mL})$ and added aqueous sodium hydroxide $(1.0 \mathrm{M})$ until $\mathrm{pH}=13.0$. The mixture was extracted with diethyl ether $(3 \times 10 \mathrm{~mL})$, and the organic layer was washed with brine, dried over anhydrous sodium sulfate, and concentrated under reduced pressure to give primary amine S3r.

To formaldehyde (330 mg, 30\% aqueous solution, $1.10 \mathrm{~mL}, 11.0 \mathrm{mmol}$ ) at $0{ }^{\circ} \mathrm{C}$ were added a solution of the above primary amine $\mathbf{S 3 r}$ in acetonitrile $(10 \mathrm{~mL})$, added portionwise $\mathrm{NaBH}_{3} \mathrm{CN}(779$ $\mathrm{mg}, 12.4 \mathrm{mmol})$, and added dropwise formic acid $(1.43 \mathrm{~g}, 1.18 \mathrm{~mL}, 31.0 \mathrm{mmol})$. The mixture was stirred at room temperature for $5 \mathrm{~h}$, and quenched with aqueous ammonium chloride solution (1.0 M, $10 \mathrm{~mL})$. The organic layer was separated and the aqueous layer was extracted with ethyl acetate $(3 \times$ $10 \mathrm{~mL}$ ). The organic layers were combined, dried over anhydrous sodium sulfate, and concentrated under reduced pressure. The organic layer was added hydrochloric acid $(1.0 \mathrm{M})$ until $\mathrm{pH}=2.0$. The aqueous layer was extracted with diethyl ether $(3 \times 10 \mathrm{~mL})$ and added aqueous sodium hydroxide $(1.0 \mathrm{M})$ until $\mathrm{pH}=13.0$. The mixture was extracted with diethyl ether $(3 \times 10 \mathrm{~mL})$, and the organic 
layer was washed with brine, dried over anhydrous sodium sulfate, and concentrated under reduced pressure to give $N, N$-dimethyl-1-(naphthalen-1-yl)-1-(thiophen-2-yl)methanamine (S4r) as a white solid (1.28 g, $48 \%$ yield for two steps). m.p. $75-76{ }^{\circ} \mathrm{C} .{ }^{1} \mathrm{H}$ NMR $\left(400 \mathrm{MHz}, \mathrm{CDCl}_{3}\right) \delta 8.43(\mathrm{~d}, J=8.8$ $\mathrm{Hz}, 1 \mathrm{H}), 7.93(\mathrm{~d}, J=7.6 \mathrm{~Hz}, 1 \mathrm{H}), 7.80(\mathrm{~d}, J=8.0 \mathrm{~Hz}, 1 \mathrm{H}), 7.72(\mathrm{~d}, J=8.0 \mathrm{~Hz}, 1 \mathrm{H}), 7.51-7.38(\mathrm{~m}$, $3 \mathrm{H}), 7.10(\mathrm{dd}, J=5.2,0.8 \mathrm{~Hz}, 1 \mathrm{H}), 7.02(\mathrm{dd}, J=7.2,0.8 \mathrm{~Hz}, 1 \mathrm{H}), 6.80(\mathrm{dd}, J=5.2,3.2 \mathrm{~Hz}, 1 \mathrm{H})$, $5.23(\mathrm{~s}, 1 \mathrm{H}), 2.28(\mathrm{~s}, 6 \mathrm{H}) ;{ }^{13} \mathrm{C} \mathrm{NMR}\left(100 \mathrm{MHz}, \mathrm{CDCl}_{3}\right) \delta 147.1,138.5,134.1,131.3,129.0,127.9$, $126.1,125.9,125.8,125.7,125.5,125.2,125.1,123.5,68.0,44.8$; HRMS (ESI) calcd for $\mathrm{C}_{17} \mathrm{H}_{18} \mathrm{NS}^{+}$ $(\mathrm{M}+\mathrm{H})^{+} 268.1154$, found 268.1162 .

\section{General Procedure for the Aromatic Substitution of Benzylic Ammonium Salts with Amines}

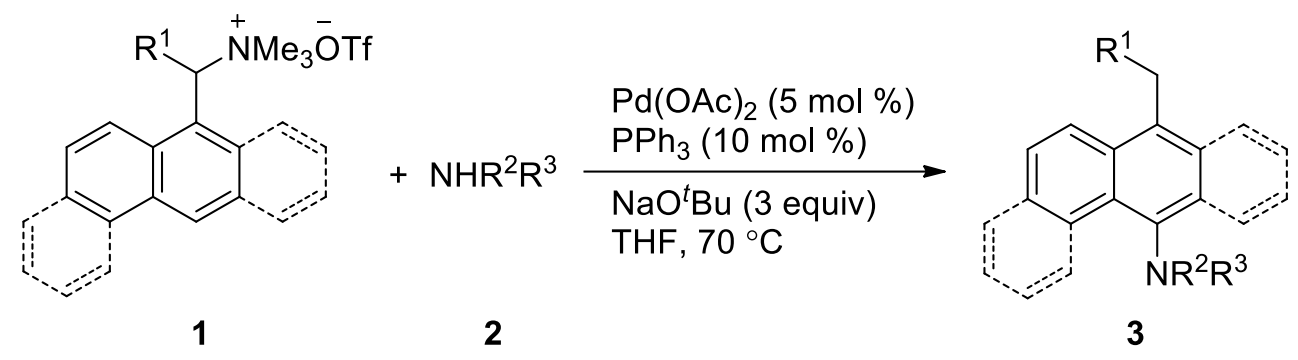

To a suspension of $\mathrm{Pd}(\mathrm{OAc})_{2}(2.2 \mathrm{mg}, 0.01 \mathrm{mmol}), \mathrm{PPh}_{3}(5.2 \mathrm{mg}, 0.02 \mathrm{mmol}), \mathrm{NaO}^{t} \mathrm{Bu}(57.7 \mathrm{mg}$, $0.60 \mathrm{mmol})$, and benzylic ammonium salt $1(0.20 \mathrm{mmol})$ in tetrahydrofuran $(1.0 \mathrm{~mL})$ under nitrogen atmosphere was added amine $2(0.20 \mathrm{mmol})$. The mixture was heated at $70{ }^{\circ} \mathrm{C}$ for $5 \mathrm{~h}$, cooled to room temperature, and purified by silica gel chromatography, using a mixture of ethyl acetate and petroleum ether $(1: 10)$ as the eluent, to give aromatic amine 3 .

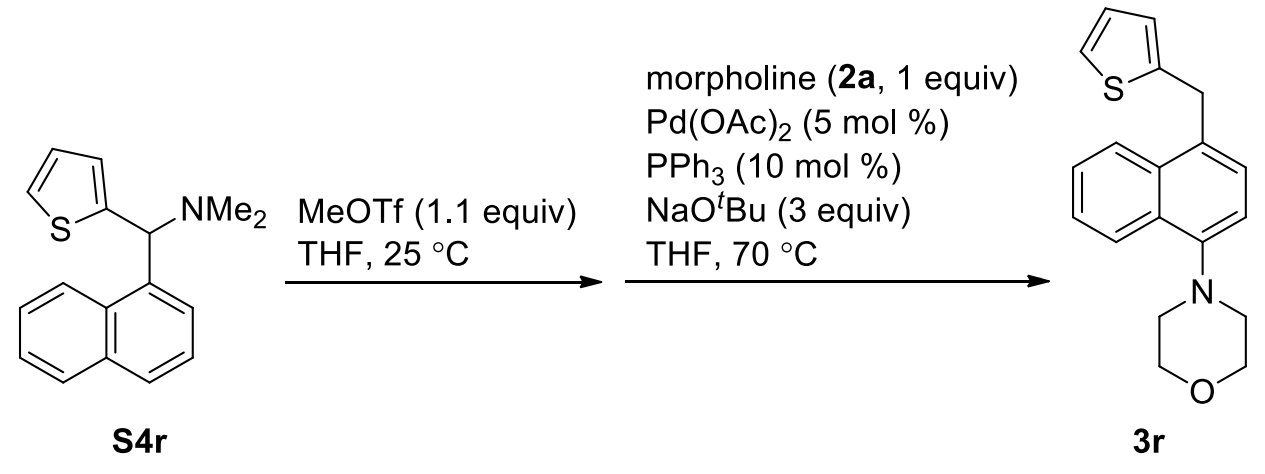

To an oven-dried $10 \mathrm{~mL}$ test tube with standard ground joint equipped with a stir bar under nitrogen atmosphere were added benzylic amine $\mathbf{S 4 r}$ (53.5 mg, $0.20 \mathrm{mmol}$ ), MeOTf (36.1 mg, 24.9 $\mu \mathrm{L}, 0.22 \mathrm{mmol})$, and tetrahydrofuran $(1.0 \mathrm{~mL})$. The mixture was stirred at room temperature for 5 min. To the mixture were added $\mathrm{Pd}(\mathrm{OAc})_{2}(2.2 \mathrm{mg}, 0.01 \mathrm{mmol}), \mathrm{PPh}_{3}(5.2 \mathrm{mg}, 0.02 \mathrm{mmol}), \mathrm{NaO}{ }^{t} \mathrm{Bu}$ $(57.7 \mathrm{mg}, 0.60 \mathrm{mmol})$, and amine $2 \mathbf{a}(17.4 \mathrm{mg}, 17.4 \mu \mathrm{L}, 0.20 \mathrm{mmol})$. The mixture was heated at 70 ${ }^{\circ} \mathrm{C}$ for $5 \mathrm{~h}$, cooled to room temperature, and purified by silica gel chromatography, using a mixture of ethyl acetate and petroleum ether (1:10) as the eluent, to give aromatic amine 3r.

\section{A 5-mmol Scale Reaction}




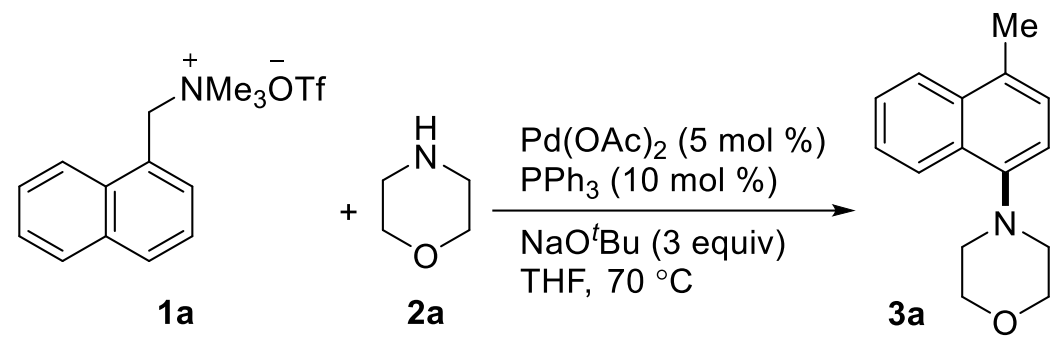

To a suspension of $\mathrm{Pd}(\mathrm{OAc})_{2}(56.1 \mathrm{mg}, 0.25 \mathrm{mmol}), \mathrm{PPh}_{3}(131 \mathrm{mg}, 0.50 \mathrm{mmol}), \mathrm{NaO}^{t} \mathrm{Bu}(1.44 \mathrm{~g}$, $15.0 \mathrm{mmol})$, and benzylic ammonium salt $1 \mathrm{a}(1.75 \mathrm{~g}, 5.0 \mathrm{mmol})$ in tetrahydrofuran $(20 \mathrm{~mL})$ under nitrogen atmosphere was added amine $2 \mathbf{a}(436 \mathrm{mg}, 0.44 \mathrm{~mL}, 5.0 \mathrm{mmol})$. The mixture was heated at $70{ }^{\circ} \mathrm{C}$ for $5 \mathrm{~h}$, cooled to room temperature, then filtered and washed with ethyl acetate $(20 \mathrm{~mL})$. The mixture was concentrated under reduced pressure, and purified by silica gel chromatography, using a mixture of ethyl acetate and petroleum ether (1:10) as the eluent, to give aromatic amine 3a as a white solid (920 mg, 81\%).

\section{Analytical Data for the Products}<smiles>Cc1ccc(N2CCOCC2)c2ccccc12</smiles>

$3 a$

4-(4-Methylnaphthalen-1-yl)morpholine (3a), ${ }^{9}$ white solid (39.1 mg, 86\% yield), m.p. $46-47{ }^{\circ} \mathrm{C}$. ${ }^{1} \mathrm{H}$ NMR $\left(400 \mathrm{MHz}, \mathrm{CDCl}_{3}\right) \delta$ 8.30-8.25 (m, 1H), 7.99-7.95 (m, 1H), 7.55-7.47 (m, 2H), 7.24 (dd, $J$ $=7.6,0.8 \mathrm{~Hz}, 1 \mathrm{H}), 7.01(\mathrm{~d}, J=7.6 \mathrm{~Hz}, 1 \mathrm{H}), 3.97(\mathrm{t}, J=4.8 \mathrm{~Hz}, 4 \mathrm{H}), 3.09-3.06(\mathrm{~m}, 4 \mathrm{H}), 2.63(\mathrm{~d}, J=$ $0.8 \mathrm{~Hz}, 3 \mathrm{H}) ;{ }^{13} \mathrm{C} \mathrm{NMR}\left(100 \mathrm{MHz}, \mathrm{CDCl}_{3}\right) \delta 148.0,133.8,130.0,129.0,126.5,125.9,125.3,124.9$, 124.0, 114.7, 67.6, 53.8, 19.3.<smiles>COc1cc(N2CCOCC2)c2ccccc2c1OC</smiles>

4-(3-Methoxy-4-methylnaphthalen-1-yl)morpholine (3b), ${ }^{9}$ white solid (42.7 $\mathrm{mg}, 83 \%$ yield), m.p. $148-149{ }^{\circ} \mathrm{C} .{ }^{1} \mathrm{H}$ NMR $\left(400 \mathrm{MHz}, \mathrm{CDCl}_{3}\right) \delta 8.18(\mathrm{~d}, J=8.4 \mathrm{~Hz}, 1 \mathrm{H}), 7.93(\mathrm{~d}, J=8.4 \mathrm{~Hz}, 1 \mathrm{H})$, 7.51-7.45 (m, 1H), 7.38-7.32 (m, 1H), $6.92(\mathrm{~s}, 1 \mathrm{H}), 3.98(\mathrm{t}, J=4.4 \mathrm{~Hz}, 4 \mathrm{H}), 3.93(\mathrm{~s}, 3 \mathrm{H}), 3.16-3.06$ $(\mathrm{m}, 4 \mathrm{H}), 2.50(\mathrm{~s}, 3 \mathrm{H}) ;{ }^{13} \mathrm{C} \mathrm{NMR}\left(100 \mathrm{MHz}, \mathrm{CDCl}_{3}\right) \delta 154.4,149.0,134.7,126.5,124.6,124.1,123.8$, $123.1,115.3,104.3,67.5,57.1,53.8,10.5$. 
<smiles>Cc1c(Br)cc(N2CCOCC2)c2ccccc12</smiles>

4-(3-Bromo-4-methylnaphthalen-1-yl)morpholine (3c), white solid (38.0 mg, $62 \%$ yield), m.p. 83-84 ${ }^{\circ} \mathrm{C} .{ }^{1} \mathrm{H}$ NMR $\left(400 \mathrm{MHz}, \mathrm{CDCl}_{3}\right) \delta 8.23-8.18(\mathrm{~m}, 1 \mathrm{H}), 8.00(\mathrm{dd}, J=7.6,1.6 \mathrm{~Hz}, 1 \mathrm{H}), 7.56-7.47$ $(\mathrm{m}, 2 \mathrm{H}), 7.21(\mathrm{~s}, 1 \mathrm{H}), 3.96(\mathrm{t}, J=4.4 \mathrm{~Hz}, 4 \mathrm{H}), 3.08-3.06(\mathrm{~m}, 4 \mathrm{H}), 2.74(\mathrm{~s}, 3 \mathrm{H}) ;{ }^{13} \mathrm{C} \mathrm{NMR}(100 \mathrm{MHz}$, $\left.\mathrm{CDCl}_{3}\right) \delta 148.6,134.4,129.0,128.1,127.0,125.4,125.2,124.2,122.6,119.7,67.5,53.7,18.7$; HRMS (ESI) calcd for $\mathrm{C}_{15} \mathrm{H}_{17} \mathrm{NBrO}^{+}(\mathrm{M}+\mathrm{H})^{+} 306.0488$, found 306.0498 .<smiles>Cc1ccc2c(N3CCOCC3)ccc(C)c2c1</smiles>

3d

4-(4,6-Dimethylnaphthalen-1-yl)morpholine (3d), white solid (37.6 mg, 78\% yield), m.p. 31-32 ${ }^{\circ} \mathrm{C} .{ }^{1} \mathrm{H}$ NMR $\left(400 \mathrm{MHz}, \mathrm{CDCl}_{3}\right) \delta 8.15(\mathrm{~d}, J=8.4 \mathrm{~Hz}, 1 \mathrm{H}), 7.73(\mathrm{~s}, 1 \mathrm{H}), 7.32(\mathrm{dd}, J=8.8,1.6$ $\mathrm{Hz}, 1 \mathrm{H}), 7.19(\mathrm{dd}, J=7.2,0.4 \mathrm{~Hz}, 1 \mathrm{H}), 6.91(\mathrm{~d}, J=7.6 \mathrm{~Hz}, 1 \mathrm{H}), 3.95(\mathrm{t}, J=4.4 \mathrm{~Hz}, 4 \mathrm{H}), 3.06-3.04$ $(\mathrm{m}, 4 \mathrm{H}), 2.60(\mathrm{~s}, 3 \mathrm{H}), 2.53(\mathrm{~s}, 3 \mathrm{H}) ;{ }^{13} \mathrm{C} \mathrm{NMR}\left(100 \mathrm{MHz}, \mathrm{CDCl}_{3}\right) \delta$ 148.0, 135.4, 134.0, 129.3, 127.4, 127.1, 126.6, 124.0, 123.9, 113.7, 67.6, 53.7, 22.0, 19.3; HRMS (ESI) calcd for $\mathrm{C}_{16} \mathrm{H}_{20} \mathrm{NO}^{+}(\mathrm{M}+\mathrm{H})^{+}$ 242.1539 , found 242.1535 .<smiles>Cc1ccc(N2CCOCC2)c2ccc(F)cc12</smiles>

$3 e$

4-(6-Fluoro-4-methylnaphthalen-1-yl)morpholine (3e), white solid (28.0 mg, 57\% yield), m.p. 86-87 ${ }^{\circ} \mathrm{C} .{ }^{1} \mathrm{H}$ NMR $\left(400 \mathrm{MHz}, \mathrm{CDCl}_{3}\right) \delta 8.28(\mathrm{dd}, J=9.2,6.0 \mathrm{~Hz}, 1 \mathrm{H}), 7.55(\mathrm{dd}, J=11.2,2.4 \mathrm{~Hz}$, $1 \mathrm{H}), 7.29-7.22(\mathrm{~m}, 2 \mathrm{H}), 6.97(\mathrm{~d}, J=7.2 \mathrm{~Hz}, 1 \mathrm{H}), 3.97(\mathrm{t}, J=4.4 \mathrm{~Hz}, 4 \mathrm{H}), 3.06(\mathrm{t}, J=4.4 \mathrm{~Hz}, 4 \mathrm{H})$, $2.57(\mathrm{~s}, 3 \mathrm{H}) ;{ }^{13} \mathrm{C}$ NMR $\left(100 \mathrm{MHz}, \mathrm{CDCl}_{3}\right) \delta 160.9\left(\mathrm{~d}, J_{\mathrm{C}-\mathrm{F}}=244.2 \mathrm{~Hz}\right), 148.2,135.0\left(\mathrm{~d}, J_{\mathrm{C}-\mathrm{F}}=8.4\right.$ $\mathrm{Hz}), 129.5\left(\mathrm{~d}, J_{\mathrm{C}-\mathrm{F}}=5.2 \mathrm{~Hz}\right), 127.7,126.7\left(\mathrm{~d}, J_{\mathrm{C}-\mathrm{F}}=8.8 \mathrm{~Hz}\right), 126.0,115.2\left(\mathrm{~d}, J_{\mathrm{C}-\mathrm{F}}=24.5 \mathrm{~Hz}\right), 114.1$, 
$108.5\left(\mathrm{~d}, J_{\mathrm{C}-\mathrm{F}}=20.7 \mathrm{~Hz}\right), 67.6,53.8,19.3 ;{ }^{19} \mathrm{~F} \mathrm{NMR}\left(376 \mathrm{MHz}, \mathrm{CDCl}_{3}\right) \delta-114.49 ; \mathrm{HRMS}(\mathrm{ESI})$ calcd for $\mathrm{C}_{15} \mathrm{H}_{17} \mathrm{NOF}^{+}(\mathrm{M}+\mathrm{H})^{+} 246.1289$, found 246.1283 .

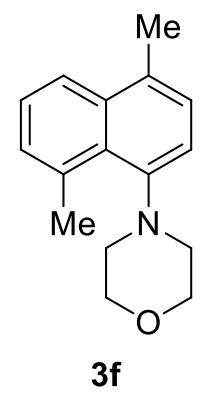

4-(4,8-Dimethylnaphthalen-1-yl)morpholine (3f), white solid (27.0 mg, 56\% yield), m.p. 91-92 ${ }^{\circ} \mathrm{C} .{ }^{1} \mathrm{H}$ NMR $\left(400 \mathrm{MHz}, \mathrm{CDCl}_{3}\right) \delta 7.82(\mathrm{~d}, J=8.0 \mathrm{~Hz}, 1 \mathrm{H}), 7.37(\mathrm{dd}, J=8.0,7.2 \mathrm{~Hz}, 1 \mathrm{H})$, 7.28-7.22 (m, 2H), 7.16 (d, J=7.6 Hz, 1H), 3.94-3.88 (m, 4H), 3.05 (s, 3H), 2.97-2.93 (m, 4H), 2.62 $(\mathrm{d}, J=0.4 \mathrm{~Hz}, 3 \mathrm{H}) ;{ }^{13} \mathrm{C} \mathrm{NMR}\left(100 \mathrm{MHz}, \mathrm{CDCl}_{3}\right) \delta 149.9,135.4,135.2,131.4,129.5,129.4,126.4$, 125.3, 123.1, 117.7, 67.1, 54.5, 25.1, 20.3; HRMS (ESI) calcd for $\mathrm{C}_{16} \mathrm{H}_{20} \mathrm{NO}^{+}(\mathrm{M}+\mathrm{H})^{+} 242.1539$, found 242.1535 .<smiles>Cc1c2ccccc2c(N2CCOCC2)c2ccccc12</smiles>

$3 g$

4-(10-Methylanthracen-9-yl)morpholine (3g), ${ }^{9}$ white solid (42.2 $\mathrm{mg}, 76 \%$ yield), m.p. 163-164 ${ }^{\circ} \mathrm{C} .{ }^{1} \mathrm{H}$ NMR $\left(400 \mathrm{MHz}, \mathrm{CDCl}_{3}\right) \delta 8.58-8.53(\mathrm{~m}, 2 \mathrm{H}), 8.32-8.26(\mathrm{~m}, 2 \mathrm{H}), 7.51-7.45(\mathrm{~m}, 4 \mathrm{H})$, $4.02(\mathrm{t}, J=4.4 \mathrm{~Hz}, 4 \mathrm{H}), 3.49(\mathrm{t}, J=4.4 \mathrm{~Hz}, 4 \mathrm{H}), 3.05(\mathrm{~s}, 3 \mathrm{H}) ;{ }^{13} \mathrm{C} \mathrm{NMR}\left(100 \mathrm{MHz}, \mathrm{CDCl}_{3}\right) \delta 142.1$, $130.9,130.4,129.1,125.5,125.2,125.1,124.9,68.5,51.7,14.2$.<smiles>Cc1ccc(N2CCOCC2)c2c1ccc1ccccc12</smiles>

$3 \mathrm{~h}$

4-(1-Methylphenanthren-4-yl)morpholine (3h), white solid (27.7 $\mathrm{mg}, 50 \%$ yield), m.p. 167-168 ${ }^{\circ} \mathrm{C} .{ }^{1} \mathrm{H}$ NMR $\left(400 \mathrm{MHz}, \mathrm{CDCl}_{3}\right) \delta 10.46(\mathrm{dd}, J=8.4,1.2 \mathrm{~Hz}, 1 \mathrm{H}), 7.89(\mathrm{~d}, J=9.2 \mathrm{~Hz}, 1 \mathrm{H})$, $7.85(\mathrm{dd}, J=8.0,2.0 \mathrm{~Hz}, 1 \mathrm{H}), 7.73(\mathrm{~d}, J=8.8 \mathrm{~Hz}, 1 \mathrm{H}), 7.62-7.51(\mathrm{~m}, 2 \mathrm{H}), 7.36(\mathrm{dd}, J=7.6,0.8 \mathrm{~Hz}$, $1 \mathrm{H}), 7.24(\mathrm{~d}, J=7.6 \mathrm{~Hz}, 1 \mathrm{H}), 4.09-4.01(\mathrm{~m}, 2 \mathrm{H}), 3.98-3.92(\mathrm{~m}, 2 \mathrm{H}), 3.21-3.16(\mathrm{~m}, 2 \mathrm{H}), 2.96-2.87(\mathrm{~m}$, 2H), 2.68 (s, 3H); $\left.{ }^{13} \mathrm{C} \mathrm{NMR} \mathrm{(100} \mathrm{MHz,} \mathrm{CDCl}_{3}\right) \delta 149.9,133.2,132.4,131.3,130.2,128.3,128.0$, 
127.6, 127.4, 126.1, 125.7, 124.0, 123.6, 116.7, 67.3, 53.0, 20.2; HRMS (ESI) calcd for $\mathrm{C}_{19} \mathrm{H}_{20} \mathrm{NO}^{+}$ $(\mathrm{M}+\mathrm{H})^{+} 278.1539$, found 278.1543 .<smiles>CCc1ccc(N2CCOCC2)c2ccccc12</smiles>

3i

4-(4-Ethylnaphthalen-1-yl)morpholine (3i), white solid (17.4 mg, 36\% yield), m.p. $98-99{ }^{\circ} \mathrm{C} .{ }^{1} \mathrm{H}$ NMR $\left(400 \mathrm{MHz}, \mathrm{CDCl}_{3}\right) \delta$ 8.24-8.19 (m, 1H), 8.00-7.93 (m, 1H), 7.47-7.38 (m, 2H), $7.20(\mathrm{~d}, J=7.6$ $\mathrm{Hz}, 1 \mathrm{H}), 6.97(\mathrm{~d}, J=7.6 \mathrm{~Hz}, 1 \mathrm{H}), 3.91(\mathrm{t}, J=4.4 \mathrm{~Hz}, 4 \mathrm{H}), 3.02-2.94(\mathrm{~m}, 6 \mathrm{H}), 1.29(\mathrm{t}, J=7.6 \mathrm{~Hz}$, $3 \mathrm{H}) ;{ }^{13} \mathrm{C}$ NMR $\left(100 \mathrm{MHz}, \mathrm{CDCl}_{3}\right) \delta 147.9,136.1,133.0,129.2,125.9,125.2,124.8,124.5,124.1$, 114.8, 67.6, 53.8, 25.8, 15.2; HRMS (ESI) calcd for $\mathrm{C}_{16} \mathrm{H}_{20} \mathrm{NO}^{+}(\mathrm{M}+\mathrm{H})^{+}$242.1539, found 242.1532 .<smiles>CC(C)Cc1ccc(N2CCOCC2)c2ccccc12</smiles>

3j

4-(4-Isobutylnaphthalen-1-yl)morpholine $(\mathbf{3 j}),{ }^{9}$ colorless oil $\left(36.6 \mathrm{mg}, 68 \%\right.$ yield). ${ }^{1} \mathrm{H}$ NMR $\left(400 \mathrm{MHz}, \mathrm{CDCl}_{3}\right) \delta 8.33-8.30(\mathrm{~m}, 1 \mathrm{H}), 8.03-7.98(\mathrm{~m}, 1 \mathrm{H}), 7.52-7.47(\mathrm{~m}, 2 \mathrm{H}), 7.21(\mathrm{~d}, J=7.6 \mathrm{~Hz}$, $1 \mathrm{H}), 7.05(\mathrm{~d}, J=6.4 \mathrm{~Hz}, 1 \mathrm{H}), 4.09-3.95(\mathrm{~m}, 4 \mathrm{H}), 3.17-3.06(\mathrm{~m}, 4 \mathrm{H}), 2.86(\mathrm{~d}, J=7.2 \mathrm{~Hz}, 2 \mathrm{H})$, 2.09-1.97 (m, 1H), $0.96(\mathrm{~d}, J=6.4 \mathrm{~Hz}, 6 \mathrm{H}) ;{ }^{13} \mathrm{C} \mathrm{NMR}\left(100 \mathrm{MHz}, \mathrm{CDCl}_{3}\right) \delta 147.5,134.0,133.4$, $129.9,127.0,125.8,125.2,124.9,124.1,114.6,67.5,53.8,42.6,29.5,23.0$.<smiles>c1ccc(Cc2ccc(N3CCOCC3)c3ccccc23)cc1</smiles>

3k

4-(4-Benzylnaphthalen-1-yl)morpholine (3k), ${ }^{9}$ white solid $\left(48.5 \mathrm{mg}, 80 \%\right.$ yield), m.p. $70-71{ }^{\circ} \mathrm{C}$. 
${ }^{1} \mathrm{H}$ NMR $\left(400 \mathrm{MHz}, \mathrm{CDCl}_{3}\right) \delta$ 8.29-8.25 (m, 1H), 7.98-7.94 (m, 1H), 7.47-7.41 (m, 2H), 7.27-7.14 $(\mathrm{m}, 6 \mathrm{H}), 7.02(\mathrm{~d}, J=7.6 \mathrm{~Hz}, 1 \mathrm{H}), 4.37(\mathrm{~s}, 2 \mathrm{H}), 3.96(\mathrm{t}, J=4.4 \mathrm{~Hz}, 4 \mathrm{H}), 3.13-3.03(\mathrm{~m}, 4 \mathrm{H}) ;{ }^{13} \mathrm{C} \mathrm{NMR}$ $\left(100 \mathrm{MHz}, \mathrm{CDCl}_{3}\right) \delta 148.6,140.9,133.3,132.3,129.3,128.8,128.5,127.4,126.1,125.3,125.0$, $124.1,114.5,67.6,53.7,38.9$.<smiles>Cc1ccc(Cc2ccc(N3CCOCC3)c3ccccc23)cc1</smiles>

31

4-(4-(4-Methylbenzyl)naphthalen-1-yl)morpholine (3l), ${ }^{9}$ white solid (52.1 mg, 82\% yield), m.p. 113-114 ${ }^{\circ} \mathrm{C} .{ }^{1} \mathrm{H}$ NMR $\left(400 \mathrm{MHz}, \mathrm{CDCl}_{3}\right) \delta$ 8.29-8.24 (m, 1H), 7.99-7.95 (m, 1H), 7.49-7.40 (m, 2H), $7.19(\mathrm{~d}, J=7.6 \mathrm{~Hz}, 1 \mathrm{H}), 7.10-7.03(\mathrm{~m}, 4 \mathrm{H}), 7.02(\mathrm{~d}, J=7.6 \mathrm{~Hz}, 1 \mathrm{H}), 4.34(\mathrm{~s}, 2 \mathrm{H}), 3.97(\mathrm{t}, J=4.8 \mathrm{~Hz}$, $4 \mathrm{H}), 3.11-3.06(\mathrm{~m}, 4 \mathrm{H}), 2.29(\mathrm{~s}, 3 \mathrm{H}) ;{ }^{13} \mathrm{C} \mathrm{NMR}\left(100 \mathrm{MHz}, \mathrm{CDCl}_{3}\right) \delta 148.5,137.8,135.6,133.3$, $132.6,129.3,129.2,128.7,127.3,126.1,125.3,125.0,124.1,114.5,67.6,53.7,38.5,21.1$.<smiles>FC(F)(F)c1ccc(Cc2ccc(N3CCOCC3)c3ccccc23)cc1</smiles>

$3 m$

4-(4-(4-(Trifluoromethyl)benzyl)naphthalen-1-yl)morpholine (3m), white solid (48.3 $\mathrm{mg}, 65 \%$ yield), m.p. $66-67{ }^{\circ} \mathrm{C} .{ }^{1} \mathrm{H}$ NMR $\left(400 \mathrm{MHz}, \mathrm{CDCl}_{3}\right) \delta 8.29$ (dd, $\left.J=8.4,1.6 \mathrm{~Hz}, 1 \mathrm{H}\right), 7.89-7.85$ (m, $1 \mathrm{H}), 7.51-7.41(\mathrm{~m}, 4 \mathrm{H}), 7.27(\mathrm{~d}, J=8.0 \mathrm{~Hz}, 2 \mathrm{H}), 7.21(\mathrm{~d}, J=7.6 \mathrm{~Hz}, 1 \mathrm{H}), 7.05(\mathrm{~d}, J=7.6 \mathrm{~Hz}, 1 \mathrm{H})$, $4.42(\mathrm{~s}, 2 \mathrm{H}), 3.98(\mathrm{t}, J=4.4 \mathrm{~Hz}, 4 \mathrm{H}), 3.14-3.17(\mathrm{~m}, 4 \mathrm{H}) ;{ }^{13} \mathrm{C} \mathrm{NMR}\left(100 \mathrm{MHz}, \mathrm{CDCl}_{3}\right) \delta 149.0$, $145.1,133.2,131.2,129.4,129.0,128.6\left(\mathrm{q}, J_{\mathrm{C}-\mathrm{F}}=20.0 \mathrm{~Hz}\right), 128.5,127.7,126.4,125.5\left(\mathrm{q}, J_{\mathrm{C}-\mathrm{F}}=3.6\right.$ $\mathrm{Hz}), 124.8,124.4\left(\mathrm{q}, J_{\mathrm{C}-\mathrm{F}}=270.2 \mathrm{~Hz}\right), 124.3,114.6,67.6,53.7,38.8 ;{ }^{19} \mathrm{~F} \mathrm{NMR}\left(376 \mathrm{MHz}, \mathrm{CDCl}_{3}\right) \delta$ -62.32 ; HRMS (ESI) calcd for $\mathrm{C}_{22} \mathrm{H}_{21} \mathrm{~F}_{3} \mathrm{NO}^{+}(\mathrm{M}+\mathrm{H})^{+} 372.1570$, found 372.1562 . 


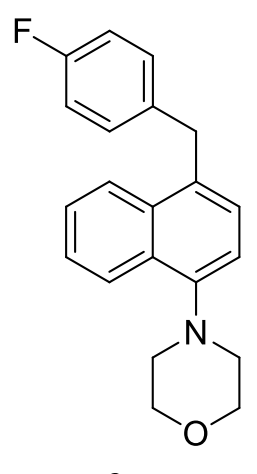

$3 n$

4-(4-(4-Fluorobenzyl)naphthalen-1-yl)morpholine (3n), ${ }^{9}$ white solid (43.1 mg, 67\% yield), m.p. 110-111 ${ }^{\circ} \mathrm{C} .{ }^{1} \mathrm{H}$ NMR $\left(400 \mathrm{MHz}, \mathrm{CDCl}_{3}\right) \delta 8.30-8.25(\mathrm{~m}, 1 \mathrm{H}), 7.94-7.89(\mathrm{~m}, 1 \mathrm{H}), 7.51-7.40(\mathrm{~m}, 2 \mathrm{H})$, $7.18(\mathrm{~d}, J=7.6 \mathrm{~Hz}, 1 \mathrm{H}), 7.15-7.10(\mathrm{~m}, 2 \mathrm{H}), 7.03(\mathrm{~d}, J=7.6 \mathrm{~Hz}, 1 \mathrm{H}), 6.96-6.90(\mathrm{~m}, 2 \mathrm{H}), 4.34(\mathrm{~s}, 2 \mathrm{H})$, $3.98(\mathrm{t}, J=4.4 \mathrm{~Hz}, 4 \mathrm{H}), 3.13-3.06(\mathrm{~m}, 4 \mathrm{H}) ;{ }^{13} \mathrm{C} \mathrm{NMR}\left(100 \mathrm{MHz}, \mathrm{CDCl}_{3}\right) \delta 161.4(\mathrm{~d}, J=242.3 \mathrm{~Hz})$, $148.7,136.5,133.2,132.1,130.1$ (d, $J=7.8 \mathrm{~Hz}), 129.3,127.3,126.2,125.4,124.9,124.2,115.3(\mathrm{~d}, J$ $=21.0 \mathrm{~Hz}), 114.5,67.6,53.7,38.2 ;{ }^{19} \mathrm{~F} \mathrm{NMR}\left(376 \mathrm{MHz}, \mathrm{CDCl}_{3}\right) \delta-117.41$.<smiles>Clc1ccc(Cc2ccc(N3CCOCC3)c3ccccc23)cc1</smiles>

30

4-(4-(4-Chlorobenzyl)naphthalen-1-yl)morpholine (3o), white solid (50.0 mg, 74\% yield), m.p. 96-97 ${ }^{\circ} \mathrm{C} .{ }^{1} \mathrm{H}$ NMR $\left(400 \mathrm{MHz}, \mathrm{CDCl}_{3}\right) \delta 8.30-8.25(\mathrm{~m}, 1 \mathrm{H}), 7.91-7.86(\mathrm{~m}, 1 \mathrm{H}), 7.51-7.40(\mathrm{~m}, 2 \mathrm{H})$, 7.23-7.17 (m, 3H), 7.09 (d, $J=8.4 \mathrm{~Hz}, 2 \mathrm{H}), 7.03(\mathrm{~d}, J=7.6 \mathrm{~Hz}, 1 \mathrm{H}), 4.33(\mathrm{~s}, 2 \mathrm{H}), 3.98(\mathrm{t}, J=4.4 \mathrm{~Hz}$, $4 \mathrm{H}), 3.12-3.06(\mathrm{~m}, 4 \mathrm{H}) ;{ }^{13} \mathrm{C} \mathrm{NMR}\left(100 \mathrm{MHz}, \mathrm{CDCl}_{3}\right) \delta 148.9,139.4,133.2,131.9,131.7,130.1$, 129.4, 128.6, 127.5, 126.2, 125.4, 124.8, 124.2, 114.5, 67.6, 53.7, 38.4; HRMS (ESI) calcd for $\mathrm{C}_{21} \mathrm{H}_{21} \mathrm{NOCl}^{+}(\mathrm{M}+\mathrm{H})^{+}$338.1306, found 338.1314.<smiles>Brc1ccc(Cc2ccc(N3CCOCC3)c3ccccc23)cc1</smiles>

4-(4-(4-Bromobenzyl)naphthalen-1-yl)morpholine (3p), ${ }^{9}$ viscous oil (61.9 mg, 81\% yield). ${ }^{1} \mathrm{H}$ 
NMR $\left(400 \mathrm{MHz}, \mathrm{CDCl}_{3}\right) \delta 8.28(\mathrm{~d}, J=8.0 \mathrm{~Hz}, 1 \mathrm{H}), 7.88(\mathrm{~d}, J=8.4 \mathrm{~Hz}, 1 \mathrm{H}), 7.50-7.41(\mathrm{~m}, 2 \mathrm{H})$, $7.36(\mathrm{~d}, J=8.4 \mathrm{~Hz}, 2 \mathrm{H}), 7.26-7.18(\mathrm{~m}, 1 \mathrm{H}), 7.07-7.02(\mathrm{~m}, 3 \mathrm{H}), 4.32(\mathrm{~s}, 2 \mathrm{H}), 3.98(\mathrm{t}, J=4.4 \mathrm{~Hz}, 4 \mathrm{H})$, 3.11-3.08 (m, 4H); $\left.{ }^{13} \mathrm{C} \mathrm{NMR} \mathrm{(100} \mathrm{MHz,} \mathrm{CDCl}_{3}\right) \delta 148.9,140.0,133.2,131.6,130.5,129.4,127.5$, $126.3,125.4,124.8,124.2,119.9,114.5,67.6,53.7,38.4$.<smiles>Brc1ccccc1Cc1ccc(N2CCOCC2)c2ccccc12</smiles>

$3 q$

4-(4-(2-Bromobenzyl)naphthalen-1-yl)morpholine (3q), white solid (61.2 mg, 80\% yield), m.p. 103-104 ${ }^{\circ} \mathrm{C} .{ }^{1} \mathrm{H}$ NMR $\left(400 \mathrm{MHz}, \mathrm{CDCl}_{3}\right) \delta 8.29(\mathrm{dd}, J=8.0,0.8 \mathrm{~Hz}, 1 \mathrm{H}), 7.85(\mathrm{~d}, J=8.0 \mathrm{~Hz}, 1 \mathrm{H})$, $7.60(\mathrm{dd}, J=8.0,1.2 \mathrm{~Hz}, 1 \mathrm{H}), 7.50-7.41(\mathrm{~m}, 2 \mathrm{H}), 7.11-7.00(\mathrm{~m}, 4 \mathrm{H}), 6.84(\mathrm{dd}, J=7.2,1.6 \mathrm{~Hz}, 1 \mathrm{H})$, $4.45(\mathrm{~s}, 2 \mathrm{H}), 3.97(\mathrm{t}, J=4.4 \mathrm{~Hz}, 4 \mathrm{H}), 3.12-3.08(\mathrm{~m}, 4 \mathrm{H}) ;{ }^{13} \mathrm{C} \mathrm{NMR}\left(100 \mathrm{MHz}, \mathrm{CDCl}_{3}\right) \delta 148.8$, $140.1,133.4,132.7,130.9,130.8,129.3,127.9,127.5,127.3,126.3,125.4,125.0,124.8,124.2$, 114.6, 67.6, 53.7, 39.0; HRMS (ESI) calcd for $\mathrm{C}_{21} \mathrm{H}_{21} \mathrm{NOBr}^{+}(\mathrm{M}+\mathrm{H})^{+}$382.0801, found 382.0794.<smiles>[Bi]C1COCCN1c1ccc(Cc2cccs2)c2ccccc12</smiles>

4-(4-(Thiophen-2-ylmethyl)naphthalen-1-yl)morpholine (3r), white solid (38.4 mg, 62\% yield), m.p. 87-88 ${ }^{\circ} \mathrm{C} .{ }^{1} \mathrm{H}$ NMR $\left(400 \mathrm{MHz}, \mathrm{CDCl}_{3}\right) \delta 8.33-8.29(\mathrm{~m}, 1 \mathrm{H}), 8.06-8.01(\mathrm{~m}, 1 \mathrm{H}), 7.53-7.46(\mathrm{~m}$, $2 \mathrm{H}), 7.32(\mathrm{~d}, J=7.6 \mathrm{~Hz}, 1 \mathrm{H}), 7.12(\mathrm{dd}, J=5.2,1.2 \mathrm{~Hz}, 1 \mathrm{H}), 7.08(\mathrm{~d}, J=6.8 \mathrm{~Hz}, 1 \mathrm{H}), 6.89(\mathrm{dd}, J=$ 4.8, 3.2 Hz, 1H), $6.76(\mathrm{dd}, J=3.6,0.8 \mathrm{~Hz}, 1 \mathrm{H}), 4.55$ (s, 2H), 4.02 (t, $J=4.0 \mathrm{~Hz}, 4 \mathrm{H}), 3.20-3.11(\mathrm{~m}$, $4 \mathrm{H}) ;{ }^{13} \mathrm{C}$ NMR $\left(100 \mathrm{MHz}, \mathrm{CDCl}_{3}\right) \delta 144.1,137.2,133.0,129.1,127.0,126.9,126.5,126.4,125.8$, 125.6, 125.3, 124.8, 124.1, 123.8, 67.4, 53.8, 33.4; HRMS (ESI) calcd for $\mathrm{C}_{19} \mathrm{H}_{20} \mathrm{NOS}^{+}(\mathrm{M}+\mathrm{H})^{+}$ 310.1260 , found 310.1252 . 


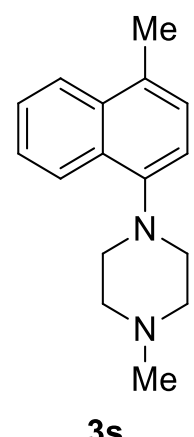

1-Methyl-4-(4-methylnaphthalen-1-yl)piperazine (3s), ${ }^{9}$ yellow solid (30.8 $\mathrm{mg}, 64 \%$ yield), m.p. 75-76 ${ }^{\circ} \mathrm{C} .{ }^{1} \mathrm{H}$ NMR $\left(400 \mathrm{MHz}, \mathrm{CDCl}_{3}\right) \delta$ 8.27-8.23 (m, $\left.1 \mathrm{H}\right), 8.00-7.93(\mathrm{~m}, 1 \mathrm{H}), 7.53-7.44(\mathrm{~m}, 2 \mathrm{H})$, $7.22(\mathrm{dd}, J=7.6,1.2 \mathrm{~Hz}, 1 \mathrm{H}), 7.00(\mathrm{~d}, J=7.6 \mathrm{~Hz}, 1 \mathrm{H}), 3.18-3.05(\mathrm{~m}, 4 \mathrm{H}), 2.71-2.64(\mathrm{~m}, 4 \mathrm{H}), 2.62$ $(\mathrm{d}, J=0.8 \mathrm{~Hz}, 3 \mathrm{H}), 2.41(\mathrm{~s}, 3 \mathrm{H}) ;{ }^{13} \mathrm{C}$ NMR $\left(100 \mathrm{MHz}, \mathrm{CDCl}_{3}\right) \delta 148.3,133.7,129.6,129.1,126.5$, $125.8,125.1,124.8,124.1,114.7,55.8,53.2,46.3,19.2$.

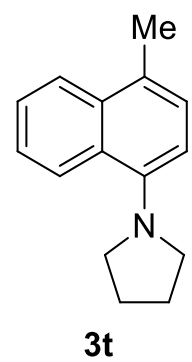

1-(4-Methylnaphthalen-1-yl)pyrrolidine (3t), ${ }^{9}$ colorless oil (22.8 mg, 54\% yield). ${ }^{1} \mathrm{H}$ NMR (400 $\left.\mathrm{MHz}, \mathrm{CDCl}_{3}\right) \delta 8.26-8.22(\mathrm{~m}, 1 \mathrm{H}), 7.96-7.91(\mathrm{~m}, 1 \mathrm{H}), 7.51-7.41(\mathrm{~m}, 2 \mathrm{H}), 7.18(\mathrm{dd}, J=7.6,0.8 \mathrm{~Hz}$, $1 \mathrm{H}), 6.92(\mathrm{~d}, J=7.6 \mathrm{~Hz}, 1 \mathrm{H}), 3.28(\mathrm{t}, J=6.4 \mathrm{~Hz}, 4 \mathrm{H}), 2.61(\mathrm{~d}, J=0.8 \mathrm{~Hz}, 3 \mathrm{H}), 2.02-1.97(\mathrm{~m}, 4 \mathrm{H})$; ${ }^{13} \mathrm{C} \mathrm{NMR}\left(100 \mathrm{MHz}, \mathrm{CDCl}_{3}\right) \delta 146.3,133.8,128.8,127.7,126.6,125.6,125.2,124.6,124.4,112.0$, 53.1, 24.6, 19.2.<smiles>Cc1ccc(N2CCCCC2)c2ccccc12</smiles>

1-(4-Methylnaphthalen-1-yl)piperidine (3u), ${ }^{9}$ white solid (35.6 mg, $79 \%$ yield), m.p. $48-49{ }^{\circ} \mathrm{C}$. ${ }^{1} \mathrm{H}$ NMR $\left(400 \mathrm{MHz}, \mathrm{CDCl}_{3}\right) \delta 8.28-8.23(\mathrm{~m}, 1 \mathrm{H}), 7.96-7.92(\mathrm{~m}, 1 \mathrm{H}), 7.50-7.46(\mathrm{~m}, 2 \mathrm{H}), 7.21(\mathrm{~d}, J=$ $6.4 \mathrm{~Hz}, 1 \mathrm{H}), 6.96(\mathrm{~d}, J=7.2 \mathrm{~Hz}, 1 \mathrm{H}), 3.20-2.81(\mathrm{~m}, 4 \mathrm{H}), 2.62(\mathrm{~s}, 3 \mathrm{H}), 1.84-1.80(\mathrm{~m}, 4 \mathrm{H}), 1.79-1.48$ $(\mathrm{m}, 2 \mathrm{H}),{ }^{13} \mathrm{C}$ NMR $\left(100 \mathrm{MHz}, \mathrm{CDCl}_{3}\right) \delta 149.7,133.7,129.3,129.0,126.5,125.7,125.0,124.7$, $124.4,114.4,54.9,26.8,24.7,19.3$. 
<smiles>Cc1ccc(N2CCC(OC(c3ccc(Cl)cc3)c3ccccn3)CC2)c2ccccc12</smiles>

(S)-2-((4-Chlorophenyl)((1-(4-methylnaphthalen-1-yl)piperidin-4-yl)oxy)methyl)pyridine (3v), colorless oil (65.6 mg, 74\% yield). ${ }^{1} \mathrm{H}$ NMR $\left(400 \mathrm{MHz}, \mathrm{CDCl}_{3}\right) \delta 8.52(\mathrm{~d}, J=4.8 \mathrm{~Hz}, 1 \mathrm{H}), 8.24-8.20$ (m, 1H), 7.95-7.91 (m, 1H), $7.67(\mathrm{t}, J=7.6 \mathrm{~Hz}, 1 \mathrm{H}), 7.59(\mathrm{~d}, J=7.6 \mathrm{~Hz}, 1 \mathrm{H}), 7.50-7.45(\mathrm{~m}, 2 \mathrm{H})$, $7.42(\mathrm{~d}, J=8.4 \mathrm{~Hz}, 2 \mathrm{H}), 7.29(\mathrm{~d}, J=8.4 \mathrm{~Hz}, 2 \mathrm{H}), 7.22-7.11(\mathrm{~m}, 2 \mathrm{H}), 6.93(\mathrm{~d}, J=7.2 \mathrm{~Hz}, 1 \mathrm{H}), 5.70$ (s, 1H), 3.74-3.58 (m, 1H), 3.36-3.22 (m, 2H), 2.84-2.70 (m, 2H), $2.60(\mathrm{~s}, 3 \mathrm{H}), 2.18-1.96(\mathrm{~m}, 4 \mathrm{H})$; ${ }^{13} \mathrm{C}$ NMR $\left(100 \mathrm{MHz}, \mathrm{CDCl}_{3}\right) \delta 162.2,149.0,148.7,140.5,137.0,133.6,133.3,132.1,129.3,129.2$, 128.6, 128.3, 126.4, 125.7, 125.1, 124.7, 124.1, 122.6, 120.7, 114.5, 81.0, 51.2, 32.5, 29.9, 19.2; HRMS (ESI) calcd for $\mathrm{C}_{28} \mathrm{H}_{28} \mathrm{~N}_{2} \mathrm{OCl}^{+}(\mathrm{M}+\mathrm{H})^{+} 443.1885$, found 443.1874 .<smiles>Cc1ccc(N(C)C)c2ccccc12</smiles>

$N, N, 4$-Trimethylnaphthalen-1-amine (3w),${ }^{10}$ colorless oil $(30.4 \mathrm{mg}, 82 \%) .{ }^{1} \mathrm{H}$ NMR (400 MHz, $\left.\mathrm{CDCl}_{3}\right) \delta 8.32-8.26(\mathrm{~m}, 1 \mathrm{H}), 7.99-7.93(\mathrm{~m}, 1 \mathrm{H}), 7.53-7.47(\mathrm{~m}, 2 \mathrm{H}), 7.24-7.20(\mathrm{~m}, 1 \mathrm{H}), 6.99(\mathrm{~d}, J=$ $7.6 \mathrm{~Hz}, 1 \mathrm{H}), 2.87(\mathrm{~s}, 6 \mathrm{H}), 2.62(\mathrm{~d}, J=0.8 \mathrm{~Hz}, 3 \mathrm{H}) ;{ }^{13} \mathrm{C} \mathrm{NMR}\left(100 \mathrm{MHz}, \mathrm{CDCl}_{3}\right) \delta 149.4,133.8$, 129.1, 129.0, 126.4, 125.7, 125.1, 124.7, 124.6, 113.9, 45.6, 19.3; HRMS (ESI) calcd for $\mathrm{C}_{13} \mathrm{H}_{16} \mathrm{~N}^{+}$ $(\mathrm{M}+\mathrm{H})^{+}$186.1277, found 186.1275 .<smiles>Cc1ccc(N(C)c2ccccc2)c2ccccc12</smiles>

$N, 4$-Dimethyl- $N$-phenylnaphthalen-1-amine $(\mathbf{3 x}),{ }^{9}$ yellow oil $\left(35.1 \mathrm{mg}, 71 \%\right.$ yield). ${ }^{1} \mathrm{H}$ NMR $\left(400 \mathrm{MHz}, \mathrm{CDCl}_{3}\right) \delta 8.03(\mathrm{~d}, J=8.4 \mathrm{~Hz}, 1 \mathrm{H}), 7.89(\mathrm{~d}, J=8.0 \mathrm{~Hz}, 1 \mathrm{H}), 7.55-7.49(\mathrm{~m}, 1 \mathrm{H}), 7.45-7.39$ $(\mathrm{m}, 1 \mathrm{H}), 7.32(\mathrm{dd}, J=7.6,0.8 \mathrm{~Hz}, 1 \mathrm{H}), 7.24(\mathrm{~d}, J=7.6 \mathrm{~Hz}, 1 \mathrm{H}), 7.20-7.10(\mathrm{~m}, 2 \mathrm{H}), 6.70(\mathrm{t}, J=7.2$ $\mathrm{Hz}, 1 \mathrm{H}), 6.58(\mathrm{dd}, J=8.0,0.8 \mathrm{~Hz}, 2 \mathrm{H}), 3.35(\mathrm{~s}, 3 \mathrm{H}), 2.70(\mathrm{~s}, 3 \mathrm{H}) ;{ }^{13} \mathrm{C} \mathrm{NMR}\left(100 \mathrm{MHz}, \mathrm{CDCl}_{3}\right) \delta$ $150.3,143.8,134.1,133.2,131.4,129.0,127.3,126.19,126.17,125.2,124.9,124.4,117.0,113.3$, $40.3,19.5$. 
<smiles>Cc1ccc(NCc2ccccc2)c2ccccc12</smiles>

3y

$N$-Benzyl-4-methylnaphthalen-1-amine (3y),${ }^{9}$ white solid $\left(15.3 \mathrm{mg}, 31 \%\right.$ yield), m.p. $37-38{ }^{\circ} \mathrm{C}$. ${ }^{1} \mathrm{H}$ NMR $\left(400 \mathrm{MHz}, \mathrm{CDCl}_{3}\right) \delta 7.96(\mathrm{dd}, J=8.4,1.2 \mathrm{~Hz}, 1 \mathrm{H}), 7.89(\mathrm{~d}, J=8.0 \mathrm{~Hz}, 1 \mathrm{H}), 7.55-7.42(\mathrm{~m}$, 4H), 7.38-7.32 (m, 2H), 7.31-7.26 (m, 1H), $7.15(\mathrm{~d}, J=7.6 \mathrm{~Hz}, 1 \mathrm{H}), 6.58(\mathrm{~d}, J=7.6 \mathrm{~Hz}, 1 \mathrm{H}), 4.48(\mathrm{~s}$, 2H), $2.58(\mathrm{~s}, 3 \mathrm{H}) ;{ }^{13} \mathrm{C} \mathrm{NMR}\left(100 \mathrm{MHz}, \mathrm{CDCl}_{3}\right) \delta 141.9,139.4,133.3,128.8,127.9,127.4,127.1$, $125.8,125.1,124.6,124.0,123.5,120.6,104.9,48.9,19.0$.

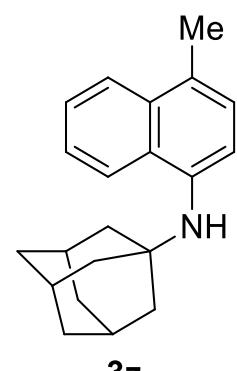

$3 z$

$\mathrm{N}$-(4-Methylnaphthalen-1-yl)adamantan-1-amine (3z), pale yellow solid (36.7 $\mathrm{mg}, 63 \%$ yield), m.p. 132-133 ${ }^{\circ} \mathrm{C} .{ }^{1} \mathrm{H}$ NMR $\left(400 \mathrm{MHz}, \mathrm{CDCl}_{3}\right) \delta 8.06(\mathrm{~d}, J=7.2 \mathrm{~Hz}, 1 \mathrm{H}), 7.96-7.91(\mathrm{~m}, 1 \mathrm{H})$, 7.51-7.44 (m, 2H), $7.14(\mathrm{~d}, J=7.6 \mathrm{~Hz}, 1 \mathrm{H}), 7.05(\mathrm{~d}, J=7.6 \mathrm{~Hz}, 1 \mathrm{H}), 2.60(\mathrm{~s}, 3 \mathrm{H}), 2.15-2.06(\mathrm{~m}, 3 \mathrm{H})$, $1.96(\mathrm{~d}, J=2.4 \mathrm{~Hz}, 6 \mathrm{H}), 1.70-1.61(\mathrm{~m}, 6 \mathrm{H}) ;{ }^{13} \mathrm{C} \mathrm{NMR}\left(100 \mathrm{MHz}, \mathrm{CDCl}_{3}\right) \delta 139.3,133.5,128.3$, 126.5, 126.4, 125.4, 125.0, 124.7, 122.2, 116.1, 53.5, 43.7, 36.6, 30.0, 19.2; HRMS (ESI) calcd for $\mathrm{C}_{21} \mathrm{H}_{26} \mathrm{~N}^{+}(\mathrm{M}+\mathrm{H})^{+}$292.2060, found 292.2051.<smiles>Cc1ccc(Nc2ccccc2)c2ccccc12</smiles>

4-Methyl- $N$-phenylnaphthalen-1-amine (3aa), ${ }^{9}$ white solid $\left(37.3 \mathrm{mg}, 80 \%\right.$ yield), m.p. $64-65{ }^{\circ} \mathrm{C}$. ${ }^{1} \mathrm{H}$ NMR $\left(400 \mathrm{MHz}, \mathrm{CDCl}_{3}\right) \delta 8.04(\mathrm{~d}, J=8.0 \mathrm{~Hz}, 1 \mathrm{H}), 8.00(\mathrm{~d}, J=8.0 \mathrm{~Hz}, 1 \mathrm{H}), 7.56-7.50(\mathrm{~m}, 1 \mathrm{H})$, 7.49-7.43 (m, 1H), 7.29-7.17 (m, 4H), 6.89-6.81 (m, 3H), $2.66(\mathrm{~s}, 3 \mathrm{H}) ;{ }^{13} \mathrm{C} \mathrm{NMR}\left(100 \mathrm{MHz}, \mathrm{CDCl}_{3}\right)$ $\delta 145.9,136.9,133.7,130.2,129.4,128.9,126.7,126.1,125.6,124.9,122.9,119.8,117.9,116.4$, 19.4 . 
<smiles>Cc1ccc(Nc2ccc(C)c3ccccc23)cc1</smiles>

4-Methyl- $N$-( $p$-tolyl)naphthalen-1-amine (3ab), ${ }^{9}$ pale yellow solid (48.5 mg, $98 \%$ yield), m.p. $50-51{ }^{\circ} \mathrm{C} .{ }^{1} \mathrm{H}$ NMR $\left(400 \mathrm{MHz}, \mathrm{CDCl}_{3}\right) \delta 8.03(\mathrm{dd}, J=8.4,0.8 \mathrm{~Hz}, 1 \mathrm{H}), 7.98(\mathrm{~d}, J=8.0 \mathrm{~Hz}, 1 \mathrm{H})$, 7.55-7.49 (m, 1H), 7.48-7.42 (m, 1H), 7.22-7.19 (m, 2H), $7.03(\mathrm{~d}, J=8.0 \mathrm{~Hz}, 2 \mathrm{H}), 6.82(\mathrm{~d}, J=8.4$ $\mathrm{Hz}, 2 \mathrm{H}), 2.64$ (s, 3H), 2.27 (s, 3H); ${ }^{13} \mathrm{C} \mathrm{NMR}\left(100 \mathrm{MHz}, \mathrm{CDCl}_{3}\right) \delta 143.0,137.7,133.6,129.9,129.6$, $129.2,128.3,126.8,126.0,125.4,124.9,122.7,117.4,116.3,20.7,19.3$.<smiles>Cc1ccc(Nc2ccc(C(C)(C)C)cc2)c2ccccc12</smiles>

$N$-(4-Chlorophenyl)-4-methylnaphthalen-1-amine (3ac), ${ }^{9}$ white solid (33.2 mg, $62 \%$ yield), m.p. 102-103 ${ }^{\circ} \mathrm{C} .{ }^{1} \mathrm{H}$ NMR $\left(400 \mathrm{MHz}, \mathrm{CDCl}_{3}\right) \delta 8.04-7.98(\mathrm{~m}, 2 \mathrm{H}), 7.58-7.52(\mathrm{~m}, 1 \mathrm{H}), 7.51-7.45(\mathrm{~m}, 1 \mathrm{H})$, $7.26-7.23(\mathrm{~m}, 2 \mathrm{H}), 7.14(\mathrm{~d}, J=8.8 \mathrm{~Hz}, 2 \mathrm{H}), 6.76(\mathrm{~d}, J=8.8 \mathrm{~Hz}, 2 \mathrm{H}), 2.68(\mathrm{~s}, 3 \mathrm{H}) ;{ }^{13} \mathrm{C}$ NMR $(100$ $\left.\mathrm{MHz}, \mathrm{CDCl}_{3}\right) \delta 144.8,136.4,133.7,130.9,129.3,129.1,126.7,126.2,125.8,125.0,124.2,122.9$, $118.7,117.3,19.4$.<smiles>Cc1ccc(Nc2ccc(Br)cc2)c2ccccc12</smiles>

$N$-(4-Bromophenyl)-4-methylnaphthalen-1-amine (3ad), ${ }^{9}$ white solid (37.5 mg, 60\% yield), m.p. $118-119{ }^{\circ} \mathrm{C} .{ }^{1} \mathrm{H}$ NMR $\left(400 \mathrm{MHz}, \mathrm{CDCl}_{3}\right) \delta$ 7.96-7.90 (m, 2H), 7.51-7.45 (m, 1H), 7.44-7.38 (m, 1H), 7.22-7.16 (m, 4H), $6.63(\mathrm{~d}, J=8.8 \mathrm{~Hz}, 2 \mathrm{H}), 2.61(\mathrm{~s}, 3 \mathrm{H}) ;{ }^{13} \mathrm{C} \mathrm{NMR}\left(100 \mathrm{MHz}, \mathrm{CDCl}_{3}\right) \delta 145.3$, $136.2,133.7,132.2,131.1,129.2,126.7,126.2,125.8,125.0,123.0,119.0,117.6,111.3,19.4$. 


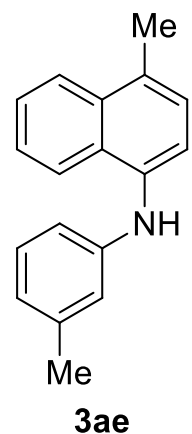

4-Methyl- $N$-( $m$-tolyl)naphthalen-1-amine (3ae) ${ }^{9}$ pale yellow solid (35.6 $\mathrm{mg}, 72 \%$ yield), m.p. 65-66 ${ }^{\circ} \mathrm{C} .{ }^{1} \mathrm{H}$ NMR $\left(400 \mathrm{MHz}, \mathrm{CDCl}_{3}\right) \delta 8.03(\mathrm{dd}, J=8.4,0.4 \mathrm{~Hz}, 1 \mathrm{H}), 7.98(\mathrm{dd}, J=8.4,0.4 \mathrm{~Hz}$, $1 \mathrm{H}), 7.54-7.48(\mathrm{~m}, 1 \mathrm{H}), 7.47-7.41(\mathrm{~m}, 1 \mathrm{H}), 7.28-7.18(\mathrm{~m}, 2 \mathrm{H}), 7.08(\mathrm{t}, J=8.0 \mathrm{~Hz}, 1 \mathrm{H}), 6.70-6.64(\mathrm{~m}$, $3 \mathrm{H}), 5.70(\mathrm{br}, 1 \mathrm{H}), 2.65(\mathrm{~s}, 3 \mathrm{H}), 2.25(\mathrm{~s}, 3 \mathrm{H}) ;{ }^{13} \mathrm{C} \mathrm{NMR}\left(100 \mathrm{MHz}, \mathrm{CDCl}_{3}\right) \delta$ 145.9, 139.3, 137.1, $133.7,129.9,129.3,128.9,126.7,126.0,125.5,124.9,122.9,120.7,117.9,117.2,113.6,21.7,19.3$.<smiles>Cc1ccccc1Nc1cccc2c(C)cccc12</smiles>

3af

4-Methyl- $N$-( $o$-tolyl)naphthalen-1-amine (3af) ${ }^{9}$ brown solid $(43.5 \mathrm{mg}, 88 \%$ yield), m.p. 51-52 ${ }^{\circ} \mathrm{C} .{ }^{1} \mathrm{H}$ NMR $\left(400 \mathrm{MHz}, \mathrm{CDCl}_{3}\right) \delta 8.05-7.99(\mathrm{~m}, 2 \mathrm{H}), 7.57-7.51(\mathrm{~m}, 1 \mathrm{H}), 7.50-7.44(\mathrm{~m}, 1 \mathrm{H})$, 7.24-7.18 (m, 2H), 7.07-7.00 (m, 2H), 6.88-6.82 (m, 1H), $6.77(\mathrm{~d}, J=8.0 \mathrm{~Hz}, 1 \mathrm{H}), 2.66(\mathrm{~s}, 3 \mathrm{H}), 2.33$ $(\mathrm{s}, 3 \mathrm{H}) ;{ }^{13} \mathrm{C}$ NMR $\left(100 \mathrm{MHz}, \mathrm{CDCl}_{3}\right) \delta 143.7,137.5,133.7,130.8,129.5,128.5,127.0,126.8,126.0$, $125.6,124.9,122.8,120.6,117.3,117.1,19.3,18.0$.<smiles>Cc1cccc(C)c1Nc1ccc(C)c2ccccc12</smiles>

$N$-(2,6-Dimethylphenyl)-4-methylnaphthalen-1-amine (3ag), ${ }^{9}$ yellow solid (31.4 mg, 60\% yield), m.p. $123-124{ }^{\circ} \mathrm{C} .{ }^{1} \mathrm{H}$ NMR $\left(400 \mathrm{MHz}, \mathrm{CDCl}_{3}\right) \delta 8.17-8.12(\mathrm{~m}, 1 \mathrm{H}), 8.02-7.98(\mathrm{~m}, 1 \mathrm{H}), 7.59-7.52(\mathrm{~m}$, $2 \mathrm{H}), 7.15(\mathrm{~d}, J=7.2 \mathrm{~Hz}, 2 \mathrm{H}), 7.11-7.02(\mathrm{~m}, 2 \mathrm{H}), 6.16$ (d, $J=7.6 \mathrm{~Hz}, 1 \mathrm{H}), 2.59$ (s, 3H), 2.19 (s, 6H); ${ }^{13} \mathrm{C}$ NMR $\left(100 \mathrm{MHz}, \mathrm{CDCl}_{3}\right) \delta 139.8,139.3,134.8,133.5,128.8,127.0,125.8,125.24,125.16$, 125.0, 124.9, 124.8, 121.2, 107.8, 19.1, 18.3; HRMS (ESI) calcd for $\mathrm{C}_{19} \mathrm{H}_{20} \mathrm{~N}^{+}(\mathrm{M}+\mathrm{H})^{+}$262.1590, found 262.1587 . 
<smiles>Cc1ccc(Nc2cccc3ccccc23)c2ccccc12</smiles>

4-Methyl- $N$-(naphthalen-1-yl)naphthalen-1-amine (3ah), white solid (32.3 mg, 57\% yield), m.p. 33-34 ${ }^{\circ} \mathrm{C} .{ }^{1} \mathrm{H}$ NMR $\left(400 \mathrm{MHz}, \mathrm{CDCl}_{3}\right) \delta 8.10(\mathrm{~d}, J=8.4 \mathrm{~Hz}, 2 \mathrm{H}), 8.04(\mathrm{~d}, J=8.0 \mathrm{~Hz}, 1 \mathrm{H}), 7.89-7.85$ $(\mathrm{m}, 1 \mathrm{H}), 7.59-7.43(\mathrm{~m}, 5 \mathrm{H}), 7.27(\mathrm{t}, J=8.0 \mathrm{~Hz}, 1 \mathrm{H}), 7.22(\mathrm{~d}, J=7.6 \mathrm{~Hz}, 1 \mathrm{H}), 7.03(\mathrm{~d}, J=7.6 \mathrm{~Hz}$, $1 \mathrm{H}), 6.82(\mathrm{~d}, J=7.2 \mathrm{~Hz}, 1 \mathrm{H}), 2.68(\mathrm{~s}, 3 \mathrm{H}) ;{ }^{13} \mathrm{C} \mathrm{NMR}\left(100 \mathrm{MHz}, \mathrm{CDCl}_{3}\right) \delta 141.4,138.2,134.7$, 133.7, 129.7, 128.8, 128.2, 126.9, 126.4, 126.2, 126.1, 125.63, 125.62, 125.0, 122.9, 121.6, 121.4, 117.6, 113.5, 19.4; HRMS (ESI) calcd for $\mathrm{C}_{21} \mathrm{H}_{18} \mathrm{~N}^{+}(\mathrm{M}+\mathrm{H})^{+} 284.1434$, found 284.1430.<smiles>Cc1ccc(Nc2cccnc2)c2ccccc12</smiles>

3ai

$N$-(4-Methylnaphthalen-1-yl)pyridin-3-amine (3ai), pale yellow solid (35.6 mg, $76 \%$ yield), m.p. 124-125 ${ }^{\circ} \mathrm{C} .{ }^{1} \mathrm{H}$ NMR $\left(400 \mathrm{MHz}, \mathrm{CDCl}_{3}\right) \delta 8.18(\mathrm{~s}, 1 \mathrm{H}), 7.96(\mathrm{t}, J=2.8 \mathrm{~Hz}, 1 \mathrm{H}), 7.91(\mathrm{~d}, J=8.8 \mathrm{~Hz}$, $2 \mathrm{H}), 7.45(\mathrm{t}, J=8.0 \mathrm{~Hz}, 1 \mathrm{H}), 7.37(\mathrm{t}, J=8.0 \mathrm{~Hz}, 1 \mathrm{H}), 7.17-7.13(\mathrm{~m}, 2 \mathrm{H}), 6.98-6.93(\mathrm{~m}, 2 \mathrm{H}), 6.05(\mathrm{~s}$, br, 1H), 2.58 (s, 3H); ${ }^{13} \mathrm{C}$ NMR (100 MHz, $\left.\mathrm{CDCl}_{3}\right) \delta 142.7,140.5,138.5,135.7,133.7,131.3,129.1$, 126.6, 126.2, 125.9, 125.0, 123.8, 122.9, 121.9, 118.9, 19.4; HRMS (ESI) calcd for $\mathrm{C}_{16} \mathrm{H}_{15} \mathrm{~N}_{2}{ }^{+}(\mathrm{M}+$ $\mathrm{H})^{+} 235.1230$, found 235.1222 .

\section{Mechanistic Studies}

(1) Isolation and Conversion of Intermediate $\mathbf{4 k}$

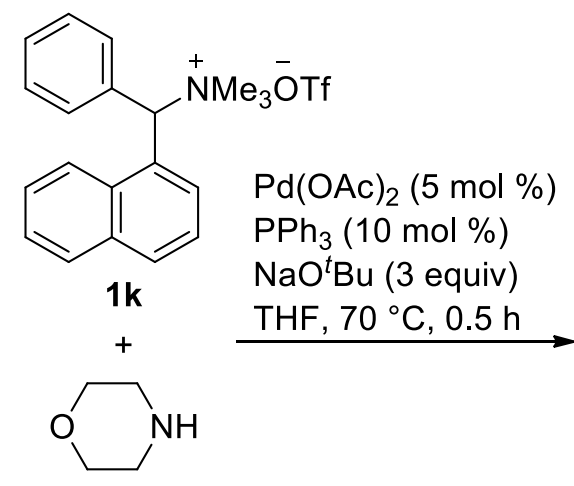

$2 a$<smiles>C1=CC(N2CCOCC2)c2ccccc2/C1=C\c1ccccc1</smiles>

$\mathrm{NaO}{ }^{t} \mathrm{Bu}$ (3 equiv) THF $70^{\circ} \mathrm{C}, 5 \mathrm{~h}$<smiles>c1ccc(Cc2ccc(N3CCOCC3)c3ccccc23)cc1</smiles>

3k 
To a suspension of $\mathrm{Pd}(\mathrm{OAc})_{2}(2.2 \mathrm{mg}, 0.01 \mathrm{mmol}), \mathrm{PPh}_{3}(5.2 \mathrm{mg}, 0.02 \mathrm{mmol}), \mathrm{NaO}^{\mathrm{t}} \mathrm{Bu}(57.7 \mathrm{mg}$, $0.60 \mathrm{mmol})$, and benzylic ammonium salts $1 \mathbf{k}(85.1 \mathrm{mg}, 0.20 \mathrm{mmol})$ in tetrahydrofuran $(1.0 \mathrm{~mL})$ under nitrogen atmosphere was added amine $2 \mathbf{a}(17.4 \mathrm{mg}, 17.4 \mu \mathrm{L}, 0.20 \mathrm{mmol})$. The resulting mixture was stirred at $70{ }^{\circ} \mathrm{C}$ for $0.5 \mathrm{~h}$, cooled to room temperature, and purified by silica gel chromatography, using a mixture of ethyl acetate and petroleum ether (1:10) as the eluent, to give 4-(4-benzylidene-1,4-dihydronaphthalen-1-yl)morpholine (4k) $)^{9}$ as a colorless oil (19.4 $\mathrm{mg}$, 32\% yield). ${ }^{1} \mathrm{H}$ NMR $\left(400 \mathrm{MHz}, \mathrm{CDCl}_{3}\right) \delta$ 7.89-7.83 (m, 1H), 7.64-7.58 (m, 1H), 7.43-7.24 (m, 8H), 7.14 $(\mathrm{d}, J=10.4 \mathrm{~Hz}, 1 \mathrm{H}), 6.18(\mathrm{ddd}, J=10.4,4.4,2.0 \mathrm{~Hz}, 1 \mathrm{H}), 4.42(\mathrm{~d}, J=4.0 \mathrm{~Hz}, 1 \mathrm{H}), 3.69-3.65(\mathrm{~m}$, $4 \mathrm{H}), 2.64-2.57(\mathrm{~m}, 2 \mathrm{H}), 2.51-2.43(\mathrm{~m}, 2 \mathrm{H}) ;{ }^{13} \mathrm{C} \mathrm{NMR}\left(100 \mathrm{MHz}, \mathrm{CDCl}_{3}\right) \delta 137.3,134.8,134.6$, $130.6,129.75,129.71,128.4,128.2,127.5,127.3,127.2,126.4,124.9,122.3,67.6,61.7,48.8$.

To a solution of compound $4 \mathbf{k}(60.7 \mathrm{mg}, 0.20 \mathrm{mmol})$ in tetrahydrofuran $(1.0 \mathrm{~mL})$ was added $\mathrm{NaO}^{t} \mathrm{Bu}(57.7 \mathrm{mg}, 0.60 \mathrm{mmol})$. The mixture was stirred at $70{ }^{\circ} \mathrm{C}$ for $5 \mathrm{~h}$, cooled to room temperature, and purified by silica gel chromatography, using a mixture of ethyl acetate and petroleum ether (1:10) as the eluent, to give aromatic amine $\mathbf{3 k}(47.3 \mathrm{mg}, 78 \%$ yield).

(2) Isolation, Conversion, and Catalytic Activity of Intermediates 5aaa and 5aab

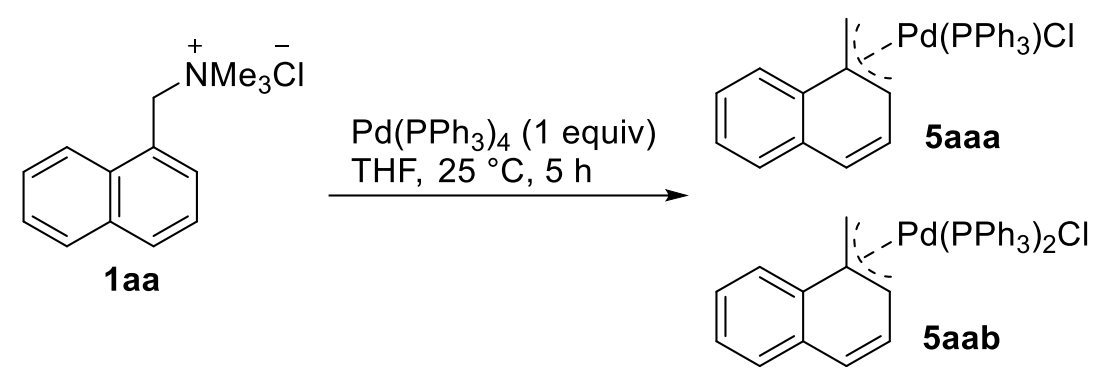

A mixture of benzylic ammonium salt 1aa $(236 \mathrm{mg}, 1.0 \mathrm{mmol})$ and $\mathrm{Pd}\left(\mathrm{PPh}_{3}\right)_{4}(1.16 \mathrm{~g}, 1.0 \mathrm{mmol})$ in tetrahydrofuran $(5 \mathrm{~mL})$ was stirred under nitrogen atpmsphere at room temperature for $5 \mathrm{~h}$. The mixture was purified by silica gel chromatography, using a mixture of methanol and dichloromethane (1:20) as the eluent, to give compounds 5aaa and 5aab.

5aaa, yellow solid (207 mg, 38\% yield), m.p. 117-120 ${ }^{\circ} \mathrm{C} .{ }^{1} \mathrm{H}$ NMR $\left(400 \mathrm{MHz}, \mathrm{CDCl}_{3}\right) \delta 7.94$ $(\mathrm{dd}, J=8.4,3.6 \mathrm{~Hz}, 1 \mathrm{H}), 7.86(\mathrm{~d}, J=7.6 \mathrm{~Hz}, 1 \mathrm{H}), 7.71(\mathrm{~d}, J=8.0 \mathrm{~Hz}, 1 \mathrm{H}), 7.67-7.58(\mathrm{~m}, 3 \mathrm{H})$, 7.57-7.49 (m, 7H), 7.43-7.34 (m, 10H), $6.41(\mathrm{t}, J=6.0 \mathrm{~Hz}, 1 \mathrm{H}) ;{ }^{13} \mathrm{C} \mathrm{NMR}\left(100 \mathrm{MHz}, \mathrm{CDCl}_{3}\right) \delta$ 135.2 , 134.2, 134.1, 132.5, 132.2, 132.14, 132.08, 130.6, 130.5, 130.4, 130.1, 130.0, 129.4, 129.0, $128.9,128.7,128.6,128.2,127.4,123.6,121.3,100.2,100.0 ;{ }^{31} \mathrm{P} \mathrm{NMR}\left(162 \mathrm{MHz}, \mathrm{CDCl}_{3}\right) \delta 30.9$; HRMS (ESI) calcd for $\mathrm{C}_{29} \mathrm{H}_{24} \mathrm{PPd}^{+}[\mathrm{M}-\mathrm{Cl}]^{+}$509.0645, found 509.0656. The structure was further confirmed by X-ray crystallographic analysis (see below).

5aab, yellow solid (258 mg, 32\% yield), m.p. 55-58 ${ }^{\circ} \mathrm{C} .{ }^{1} \mathrm{H}$ NMR (400 $\left.\mathrm{MHz}, \mathrm{CDCl}_{3}\right) \delta$ 7.76-7.64 (m, 5H), 7.38-7.27 (m, 17H), 7.27-7.23 (m, 4H), 7.13-7.07 (m, 6H), 6.80-6.74 (m, 5H), 6.55-6.51 (m, 1H), 6.14-6.09 (m, 1H); $\left.{ }^{13} \mathrm{C} \mathrm{NMR} \mathrm{(100} \mathrm{MHz,} \mathrm{CDCl}_{3}\right) \delta 135.2,135.1,133.9,133.7$, $133.6,132.3,132.2,131.2,130.7,130.3,129.9,129.7,129.2,129.0,128.9,128.8,128.7,128.6$, 128.4, 128.2, 124.4, 123.6; ${ }^{31} \mathrm{P}$ NMR (162 MHz, $\left.\mathrm{CDCl}_{3}\right) \delta 31.0(\mathrm{~d}, J=43.1 \mathrm{~Hz}), 23.0(\mathrm{~d}, J=43.1$ $\mathrm{Hz}$ ); HRMS (ESI) calcd for $\mathrm{C}_{47} \mathrm{H}_{39} \mathrm{P}_{2} \mathrm{Pd}^{+}[\mathrm{M}-\mathrm{Cl}]^{+} 771.1556$, found 771.1580. 


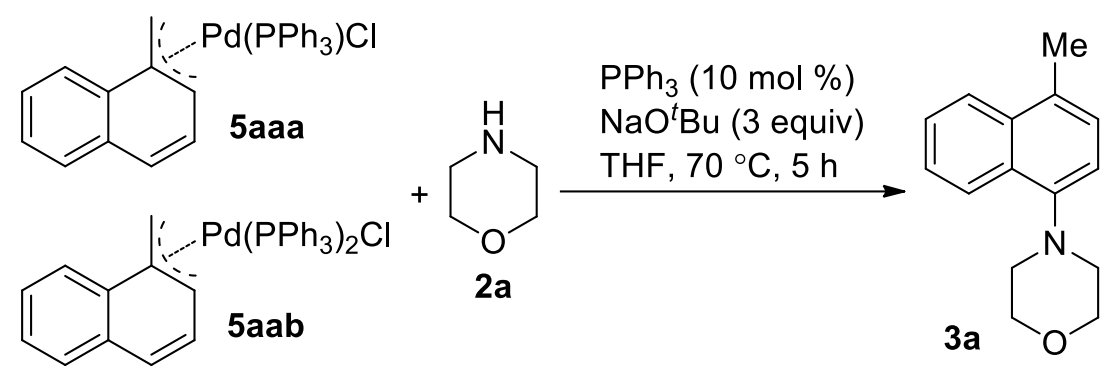

A mixture of 5 aaa $(109 \mathrm{mg}, 0.20 \mathrm{mmol}), \mathrm{NaO}^{t} \mathrm{Bu}(57.7 \mathrm{mg}, 0.60 \mathrm{mmol}), \mathrm{PPh}_{3}(5.2 \mathrm{mg}, 0.02$ $\mathrm{mmol})$, and amine $\mathbf{2 a}(17.4 \mathrm{mg}, 17.4 \mu \mathrm{L}, 0.20 \mathrm{mmol})$ in tetrahydrofuran $(1.0 \mathrm{~mL})$ was stirred under argon at $70{ }^{\circ} \mathrm{C}$ for $5 \mathrm{~h}$, and then cooled to room temperature. The mixture was concentrated under reduced pressure, and the residue was purified by silica gel chromatography, using a mixture of ethyl acetate and petroleum ether (1:10) as the eluent, to give aromatic amine 3a (17.3 $\mathrm{mg}, 38 \%$ yield).

Replacing 5aaa with $\mathbf{5 a a b}(161 \mathrm{mg}, 0.20 \mathrm{mmol})$ in the above procedure gave aromatic amine 3a (15.4 mg, 34\% yield).

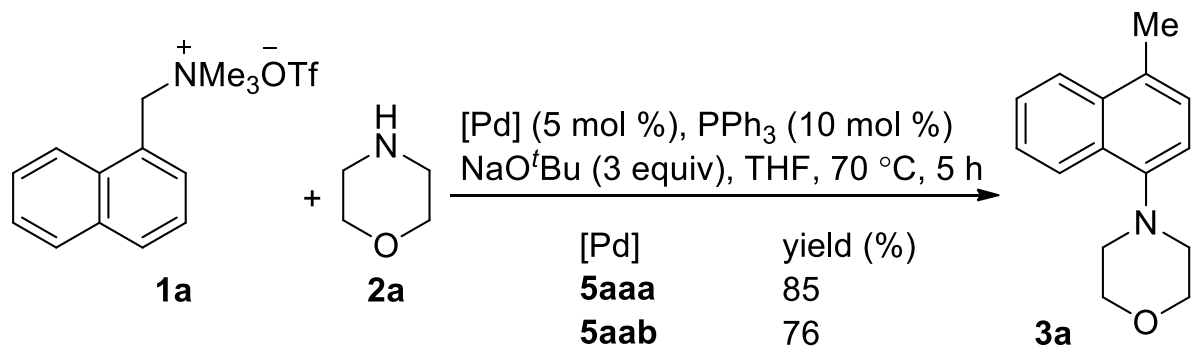

A mixture of benzylic ammonium salt $1 \mathbf{a}(69.8 \mathrm{mg}, 0.20 \mathrm{mmol}), \mathrm{PPh}_{3}(5.2 \mathrm{mg}, 0.02 \mathrm{mmol})$, $\mathrm{NaO}^{\mathrm{t}} \mathrm{Bu}(57.7 \mathrm{mg}, 0.60 \mathrm{mmol})$, 5aaa $(5.5 \mathrm{mg}, 0.01 \mathrm{mmol})$, and amine 2a $(17.4 \mathrm{mg}, 17.4 \mu \mathrm{L}, 0.20$ $\mathrm{mmol})$ in tetrahydrofuran $(1.0 \mathrm{~mL})$ was stirred under nitrogen atmosphere at $70{ }^{\circ} \mathrm{C}$ for $5 \mathrm{~h}$, and then cooled to room temperature. The mixture was concentrated under reduced pressure and the residue was purified by silica gel chromatography, using a mixture of ethyl acetate and petroleum ether (1:10) as the eluent, to give aromatic amine $\mathbf{3 a}(38.6 \mathrm{mg}, 85 \%)$.

Replacing 5 aaa with $\mathbf{5 a a b}(8.1 \mathrm{mg}, 0.01 \mathrm{mmol})$ in the above procedure gave aromatic amine 3a (34.5 mg, 76\%).

\section{References}

(1) Arnold, B.; Donald, L.; Jurgens, A.; Pincock, J. A. K. Can. J. Chem. 1985, 63, 3140-3146.

(2) Petti, M. A.; Shepodd, T. J.; Barrans, Jr, R. E.; Dougherty, D. A. J. Am. Chem. Soc. 1988, $110,6825-6840$.

(3) (a) Grudzień, K.; Żukowska, K.; Malińska, M.; Woźniak, K.; Barbasiewicz, M. Chem. Eur. J. 2014, 20, 2819-2828. (b) Li, B.; Shen, N.; Fan, X.; Zhang, X. Tetrahedron Lett. 2016, 57, $1843-1846$.

(4) Stanetty, P.; Wallner, H. Arch. Pharm. 1993, 326, 341-350.

(5) Wang, Y.; Xu, Y.-N.; Fang, G.-S.; Kang, H.-J.; Gu, Y.; Tian, S.-K. Org. Biomol. Chem. 2015, $13,5367-5371$.

(6) Maity, P.; Shacklady-McAtee, D. M.; Yap, G. P. A.; Sirianni, E. R.; Watson, M. P. J. Am. Chem. Soc. 2013, 135, 280-285.

(7) Liao, L.-Li.; Cao, G.-M.; Ye, J.-H.; Sun, G.-Q.; Zhou, W.-J.; Gui, Y.-Y.; Yan, S.-S.; Shen, G.; Yu, D.-G. J. Am. Chem. Soc. 2018, 140, 17338-17342. 
(8) Reich, H. J.; Goldenberg, W. S.; Gudmundsson, B. Ö.; Sanders, A. W.; Kulicke, K. J.; Simon, K.; Guzei, I. A. J. Am. Chem. Soc. 2001, 123, 8067-8079.

(9) Zhang, S.; Wang, Y.; Feng, X.; Bao, M. J. Am. Chem. Soc. 2012, 134, 5492-5495.

(10) Shi, R.; Lu, L.; Xie, H.; Yan, J.; Xu, T.; Zhang, H.; Qi, X.; Lan, L.; Lei, A. Chem. Commun. 2016, 52, 13307-13310.

\section{Crystallographic Data}

The crystal of compound 5aaa was obtained by leaving alone its solution in hexane and dichloromethane at room temperature in the open air for five days. The crystal data of compound 5aaa have been deposited in CCDC with number 1907865.
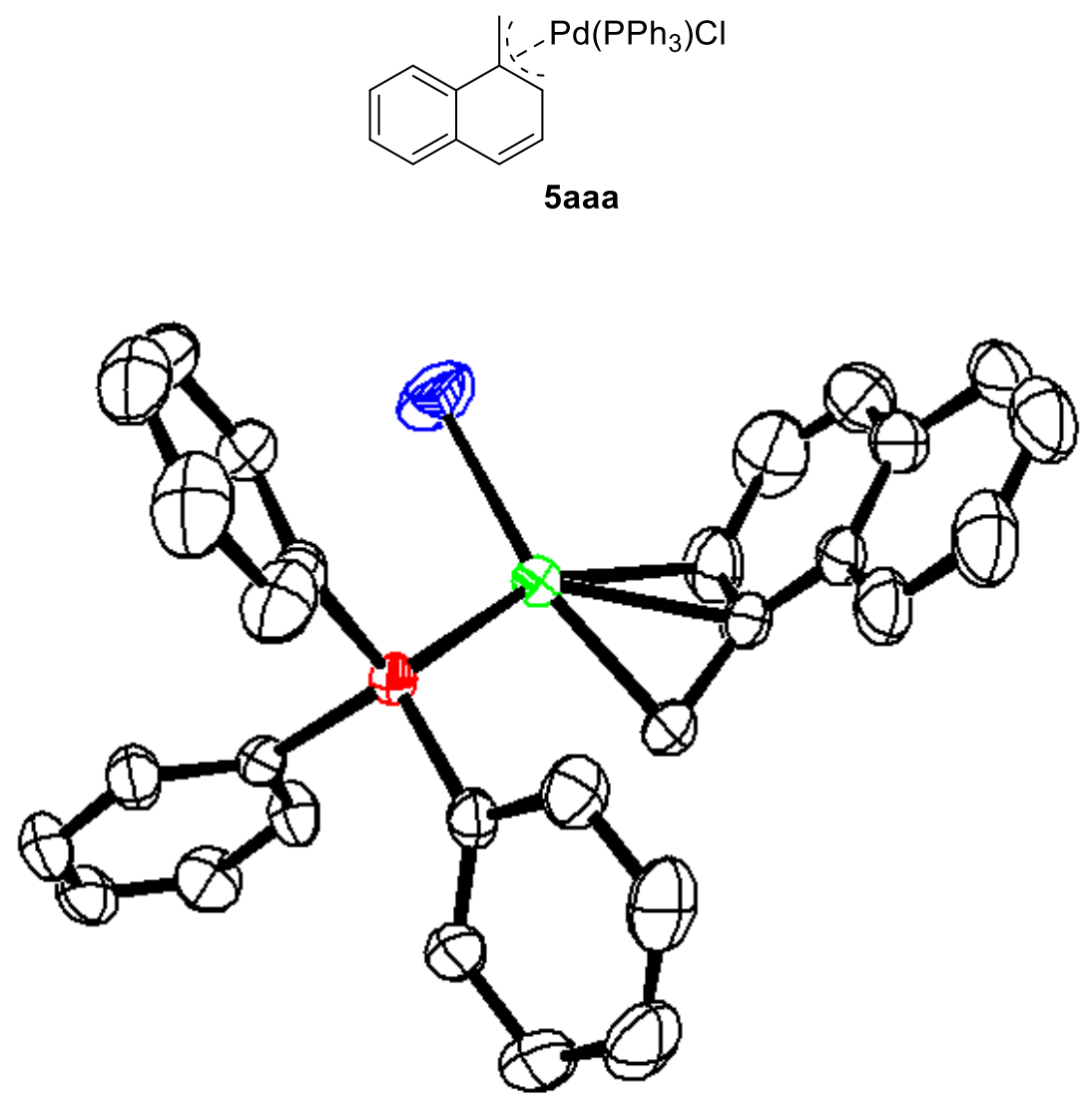

Table 1 Crystal data and structure refinement for XYN09-Cl-111.

Identification code

Empirical formula

Formula weight

Temperature/K

Crystal system

Space group

$\mathrm{a} / \AA$

$\mathrm{b} / \AA$

$\mathrm{c} / \AA$

$\alpha /{ }^{\circ}$
XYN09-Cl-111

$\mathrm{C}_{29} \mathrm{H}_{24} \mathrm{ClPPd}$

$$
545.30
$$

293(2)

monoclinic

P 1 21/n 1

9.0903(6)

28.2385(19)

$10.3361(7)$

90 


\begin{tabular}{|c|c|}
\hline$\beta /{ }^{\circ}$ & $113.741(8)$ \\
\hline$\gamma /{ }^{\circ}$ & 90 \\
\hline Volume $/ \AA^{3}$ & $2428.7(3)$ \\
\hline $\mathrm{Z}$ & 4 \\
\hline$\rho_{\text {calc }} g / \mathrm{cm}^{3}$ & 1.491 \\
\hline$\mu / \mathrm{mm}^{-1}$ & 0.955 \\
\hline $\mathrm{F}(000)$ & 1104 \\
\hline Crystal size $/ \mathrm{mm}^{3}$ & $0.330 \times 0.310 \times 0.240$ \\
\hline Radiation & $\operatorname{MoKla}(\lambda=0.71073)$ \\
\hline \multicolumn{2}{|c|}{$2 \Theta$ range for data collection $/{ }^{\circ} 7.67$ to 56.85} \\
\hline Index ranges & $-11 \leq \mathrm{h} \leq 12,-23 \leq \mathrm{k} \leq 38,-10 \leq 1 \leq 13$ \\
\hline Reflections collected & 13146 \\
\hline Independent reflections & $5800[$ Rint $=0.0352$, Rsigma $=0.0476]$ \\
\hline Data/restraints/parameters & $5800 / 0 / 289$ \\
\hline Goodness-of-fit on $\mathrm{F}^{2}$ & 1.093 \\
\hline Final $R$ indexes $[\mathrm{I}>=2 \sigma(\mathrm{I})]$ & $\mathrm{R}_{1}=0.0432, \mathrm{wR}_{2}=0.0786$ \\
\hline Final R indexes [all data] & $\mathrm{R}_{1}=0.0631, \mathrm{w} \mathrm{R}_{2}=0.0873$ \\
\hline Largest diff. peak/hole / e $\AA$ & $-3 \quad 0.51 /-0.52$ \\
\hline
\end{tabular}

Table 2 Fractional Atomic Coordinates $\left(\times 10^{4}\right)$ and Equivalent Isotropic Displacement Parameters $\left(\AA^{2} \times 10^{3}\right)$ for XYN09-Cl-111. Ueq is defined as $1 / 3$ of of the trace of the orthogonalised UIJ tensor.

\begin{tabular}{lllll} 
Atom & \multicolumn{1}{c}{$\boldsymbol{x}$} & $\boldsymbol{y}$ & $\boldsymbol{z}$ & $\mathbf{U}(\mathbf{e q})$ \\
Pd1 & $6730.9(3)$ & $5814.5(2)$ & $1534.9(2)$ & $39.85(9)$ \\
P1 & $6004.7(9)$ & $6529.0(3)$ & $2099.9(8)$ & $34.05(17)$ \\
C11 & $6172.6(16)$ & $5856.7(4)$ & $-915.0(10)$ & $82.9(3)$ \\
C24 & $6767(4)$ & $6610.3(10)$ & $4012(3)$ & $38.6(7)$ \\
C18 & $6620(3)$ & $7053.3(11)$ & $1415(3)$ & $41.3(7)$ \\
C29 & $5774(4)$ & $6546.2(13)$ & $4723(3)$ & $51.0(8)$ \\
C25 & $8400(4)$ & $6653.9(13)$ & $4803(4)$ & $54.4(9)$ \\
C13 & $3093(4)$ & $7036.2(12)$ & $1169(4)$ & $50.9(8)$ \\
C23 & $6336(4)$ & $7053.8(13)$ & $-5(4)$ & $57.3(9)$ \\
C12 & $3839(3)$ & $6603.4(10)$ & $1520(3)$ & $35.1(6)$ \\
C17 & $2923(4)$ & $6212.4(11)$ & $1532(4)$ & $48.6(8)$ \\
C2 & $8293(4)$ & $5263.1(11)$ & $3015(4)$ & $47.3(8)$ \\
C1 & $7302(4)$ & $5534.5(12)$ & $3519(4)$ & $49.6(8)$ \\
C11 & $10020(4)$ & $5322.6(12)$ & $3508(4)$ & $52.0(9)$ \\
C14 & $1471(4)$ & $7077.7(14)$ & $842(4)$ & $59.6(10)$ \\
C15 & $580(4)$ & $6687.6(15)$ & $865(4)$ & $58.1(10)$ \\
C19 & $7306(4)$ & $7444.9(13)$ & $2212(4)$ & $63.9(10)$ \\
C20 & $7687(5)$ & $7842.3(15)$ & $1597(6)$ & $83.6(14)$ \\
C16 & $1310(4)$ & $6257.3(15)$ & $1224(4)$ & $61.9(10)$ \\
C21 & $7369(5)$ & $7839.0(17)$ & $192(6)$ & $82.0(15)$
\end{tabular}




$\begin{array}{lllll}\text { C26 } & 9019(5) & 6629.4(14) & 6268(4) & 68.8(11) \\ \text { C28 } & 6396(5) & 6512.6(15) & 6181(4) & 68.8(11) \\ \text { C27 } & 8010(6) & 6553.2(15) & 6941(4) & 74.5(12) \\ \text { C6 } & 10845(5) & 5076.7(15) & 2816(5) & 69.7(12) \\ \text { C10 } & 10903(4) & 5608.6(14) & 4655(4) & 63.0(10) \\ \text { C9 } & 12541(5) & 5654.5(16) & 5102(5) & 84.1(14) \\ \text { C7 } & 12540(6) & 5126.3(19) & 3313(6) & 88.5(16) \\ \text { C3 } & 7490(5) & 4983.8(13) & 1832(4) & 68.9(11) \\ \text { C22 } & 6701(5) & 7453.2(18) & -607(5) & 77.8(13) \\ \text { C8 } & 13323(6) & 5411(2) & 0.4420(7) & 97.5(19) \\ \text { C5 } & 9987(7) & 4788.6(19) & 0.1662(6) & 98.7(17) \\ \text { C4 } & 8375(8) & 4745.1(17) & 0.1146(5) & 98.5(17)\end{array}$

Table 3 Anisotropic Displacement Parameters $\left(\AA^{2} \times 10^{3}\right)$ for XYN09-Cl-111.. The Anisotropic displacement factor exponent takes the form: $-2 \pi^{2}\left[h^{2} a^{* 2} U_{11}+2 h k a * b * U_{12}+\ldots\right]$.

\begin{tabular}{lllllll} 
Atom & \multicolumn{1}{c}{$\mathbf{U 1 1}$} & \multicolumn{1}{c}{$\mathbf{U 2 2}$} & \multicolumn{1}{c}{$\mathbf{U 3 3}$} & \multicolumn{1}{c}{$\mathbf{U 2 3}$} & \multicolumn{1}{c}{$\mathbf{U 1 3}$} & $\mathbf{U 1 2}$ \\
Pd1 & $39.76(14)$ & $42.28(14)$ & $40.20(15)$ & $2.89(11)$ & $18.91(11)$ & $5.40(11)$ \\
P1 & $30.8(4)$ & $37.5(4)$ & $33.9(4)$ & $6.0(3)$ & $13.0(3)$ & $4.2(3)$ \\
C11 & $124(10)$ & $82(8)$ & $44.5(5)$ & $0.2(5)$ & $36.0(6)$ & $5.8(7)$ \\
C24 & $39.6(16)$ & $34.6(15)$ & $37.1(17)$ & $1(13)$ & $10.6(13)$ & $7.8(13)$ \\
C18 & $27.9(15)$ & $46.1(18)$ & $50.8(19)$ & $12.6(16)$ & $16.7(14)$ & $4.9(14)$ \\
C29 & $46.8(19)$ & $67(2)$ & $37(18)$ & $1.9(17)$ & $14.9(15)$ & $9.5(17)$ \\
C25 & $44.5(19)$ & $59(2)$ & $52(2)$ & $0.5(18)$ & $11.6(16)$ & $-5.1(17)$ \\
C13 & $44.6(19)$ & $48(19)$ & $60(2)$ & $0.94(17)$ & $20.9(17)$ & $8.6(16)$ \\
C23 & $56(2)$ & $61(2)$ & $62(2)$ & $23.4(19)$ & $31.9(19)$ & $12.6(18)$ \\
C12 & $32.9(15)$ & $41.5(16)$ & $29.2(15)$ & $1.5(13)$ & $10.6(12)$ & $3.3(13)$ \\
C17 & $36.7(17)$ & $41.7(18)$ & $63(2)$ & $2.8(16)$ & $15.0(16)$ & $2.5(15)$ \\
C2 & $60(2)$ & $35.9(16)$ & $51(2)$ & $14.2(16)$ & $27.6(17)$ & $11.9(16)$ \\
C1 & $55(2)$ & $47.8(19)$ & $52(2)$ & $18.0(16)$ & $27.7(17)$ & $8.1(16)$ \\
C11 & $57(2)$ & $44.7(19)$ & $61(2)$ & $26.5(18)$ & $31.2(19)$ & $21.5(17)$ \\
C14 & $48(2)$ & $67(2)$ & $62(2)$ & $9(2)$ & $19.2(18)$ & $24.0(19)$ \\
C15 & $34.3(17)$ & $86(3)$ & $51(2)$ & $1(2)$ & $14.5(16)$ & $9.8(19)$ \\
C19 & $55(2)$ & $60(2)$ & $69(3)$ & $12(2)$ & $17.3(19)$ & $-12.5(19)$ \\
C20 & $62(3)$ & $59(3)$ & $117(4)$ & $20(3)$ & $23(3)$ & $-14(2)$ \\
C16 & $41.8(19)$ & $72(3)$ & $72(3)$ & $3(2)$ & $22.6(18)$ & $-10.5(19)$ \\
C21 & $51(2)$ & $79(3)$ & $119(4)$ & $49(3)$ & $38(3)$ & $1(2)$ \\
C26 & $53(2)$ & $70(3)$ & $54(2)$ & $7(2)$ & $-8.6(19)$ & $5(2)$ \\
C28 & $73(3)$ & $93(3)$ & $41(2)$ & $4(2)$ & $24(2)$ & $8(2)$ \\
C27 & $87(3)$ & $84(3)$ & $37(2)$ & $5(2)$ & $9(2)$ & $6(3)$ \\
C6 & $82(3)$ & $65(2)$ & $79(3)$ & $30(2)$ & $49(2)$ & $36(2)$ \\
C10 & $57(2)$ & $54(2)$ & $75(3)$ & $22(2)$ & $23(2)$ & $12.6(19)$ \\
C9 & $62(3)$ & $71(3)$ & $107(4)$ & $35(3)$ & $21(3)$ & $8(2)$ \\
C7 & $82(3)$ & $92(4)$ & $118(4)$ & $59(3)$ & $68(3)$ & $49(3)$
\end{tabular}




$\begin{array}{lllllll}\text { C3 } & 78(3) & 48(2) & 73(3) & 1(2) & 22(2) & 13(2) \\ \text { C22 } & 67(3) & 95(3) & 85(3) & 46(3) & 44(2) & 18(3) \\ \text { C8 } & 67(3) & 96(4) & 141(5) & 69(4) & 55(4) & 29(3) \\ \text { C5 } & 129(5) & 100(4) & 89(4) & 14(3) & 66(4) & 60(4) \\ \text { C4 } & 135(5) & 75(3) & 76(3) & 19(3) & 32(3) & 26(3)\end{array}$

Table 4 Bond Lengths for XYN09-Cl-111

\begin{tabular}{|c|c|c|c|c|c|}
\hline Atom & Atom & Length/Å & Atom & Atom & Length/Å \\
\hline Pd1 & $\mathrm{P} 1$ & $2.2711(8)$ & $\mathrm{C} 2$ & $\mathrm{C} 1$ & $1.431(4)$ \\
\hline Pd1 & $\mathrm{Cl1}$ & $2.3768(10)$ & $\mathrm{C} 2$ & $\mathrm{C} 11$ & $1.452(5)$ \\
\hline Pd1 & $\mathrm{C} 2$ & $2.243(3)$ & $\mathrm{C} 2$ & $\mathrm{C} 3$ & $1.389(5)$ \\
\hline Pd1 & $\mathrm{C} 1$ & $2.060(3)$ & $\mathrm{C} 11$ & C6 & $1.410(5)$ \\
\hline Pd1 & $\mathrm{C} 3$ & $2.429(4)$ & C11 & $\mathrm{C} 10$ & $1.391(5)$ \\
\hline P1 & $\mathrm{C} 24$ & $1.827(3)$ & $\mathrm{C} 14$ & $\mathrm{C} 15$ & $1.373(5)$ \\
\hline P1 & C18 & $1.823(3)$ & C15 & $\mathrm{C} 16$ & $1.363(5)$ \\
\hline P1 & C12 & $1.825(3)$ & C19 & $\mathrm{C} 20$ & $1.400(5)$ \\
\hline $\mathrm{C} 24$ & $\mathrm{C} 29$ & $1.385(4)$ & $\mathrm{C} 20$ & $\mathrm{C} 21$ & $1.361(6)$ \\
\hline $\mathrm{C} 24$ & $\mathrm{C} 25$ & $1.382(4)$ & $\mathrm{C} 21$ & $\mathrm{C} 22$ & $1.354(6)$ \\
\hline C18 & $\mathrm{C} 23$ & $1.384(5)$ & $\mathrm{C} 26$ & $\mathrm{C} 27$ & $1.373(6)$ \\
\hline $\mathrm{C} 18$ & C19 & $1.370(5)$ & $\mathrm{C} 28$ & $\mathrm{C} 27$ & $1.362(6)$ \\
\hline $\mathrm{C} 29$ & $\mathrm{C} 28$ & $1.383(4)$ & C6 & $\mathrm{C} 7$ & $1.421(6)$ \\
\hline $\mathrm{C} 25$ & $\mathrm{C} 26$ & $1.388(5)$ & C6 & $\mathrm{C} 5$ & $1.394(7)$ \\
\hline C13 & $\mathrm{C} 12$ & $1.374(4)$ & $\mathrm{C} 10$ & C9 & $1.376(5)$ \\
\hline C13 & C14 & $1.379(4)$ & $\mathrm{C} 9$ & $\mathrm{C} 8$ & $1.371(7)$ \\
\hline $\mathrm{C} 23$ & $\mathrm{C} 22$ & $1.391(5)$ & $\mathrm{C} 7$ & $\mathrm{C} 8$ & $1.344(7)$ \\
\hline C12 & C17 & $1.386(4)$ & $\mathrm{C} 3$ & $\mathrm{C} 4$ & $1.437(6)$ \\
\hline $\mathrm{C} 17$ & C16 & $1.375(4)$ & $\mathrm{C} 5$ & $\mathrm{C} 4$ & $1.348(7)$ \\
\hline
\end{tabular}

Table 5 Bond Angles for XYN09-Cl-111.

\begin{tabular}{|c|c|c|c|c|c|c|c|}
\hline Atom & Atom & Atom & Angle $/^{\circ}$ & Atom & Atom & Atom & Angle $/^{\circ}$ \\
\hline $\mathrm{P} 1$ & $\mathrm{Pd} 1$ & $\mathrm{Cl1}$ & $105.66(3)$ & $\mathrm{C} 1$ & $\mathrm{C} 2$ & C11 & $124.0(3)$ \\
\hline P1 & $\mathrm{Pd} 1$ & $\mathrm{C} 3$ & $157.27(10)$ & $\mathrm{C} 11$ & $\mathrm{C} 2$ & $\mathrm{Pd} 1$ & $117.2(2)$ \\
\hline $\mathrm{Cl1}$ & Pd1 & C3 & $96.39(10)$ & C3 & $\mathrm{C} 2$ & Pd1 & $80.2(2)$ \\
\hline $\mathrm{C} 2$ & $\mathrm{Pd} 1$ & $\mathrm{P} 1$ & $127.67(9)$ & $\mathrm{C} 3$ & $\mathrm{C} 2$ & $\mathrm{C} 1$ & 116.1(3) \\
\hline $\mathrm{C} 2$ & $\mathrm{Pd} 1$ & $\mathrm{Cl1}$ & $124.49(9)$ & C3 & $\mathrm{C} 2$ & C11 & 119.1(3) \\
\hline $\mathrm{C} 2$ & $\operatorname{Pd} 1$ & $\mathrm{C} 3$ & $34.30(12)$ & $\mathrm{C} 2$ & $\mathrm{C} 1$ & $\mathrm{Pd} 1$ & $77.66(19)$ \\
\hline $\mathrm{C} 1$ & $\mathrm{Pd} 1$ & $\mathrm{P} 1$ & $93.90(10)$ & C6 & $\mathrm{C} 11$ & $\mathrm{C} 2$ & $119.0(4)$ \\
\hline $\mathrm{C} 1$ & Pd1 & $\mathrm{Cl1}$ & $160.06(10)$ & $\mathrm{C} 10$ & $\mathrm{C} 11$ & $\mathrm{C} 2$ & $122.6(3)$ \\
\hline $\mathrm{C} 1$ & $\operatorname{Pd} 1$ & $\mathrm{C} 2$ & $38.55(12)$ & C10 & $\mathrm{C} 11$ & C6 & $118.4(4)$ \\
\hline $\mathrm{C} 1$ & Pd1 & C3 & $63.79(13)$ & $\mathrm{C} 15$ & C14 & C13 & $120.5(3)$ \\
\hline $\mathrm{C} 24$ & $\mathrm{P} 1$ & Pd1 & 111.54(9) & C16 & C15 & C14 & $119.6(3)$ \\
\hline C18 & P1 & $\mathrm{Pd} 1$ & $117.08(10)$ & C18 & C19 & $\mathrm{C} 20$ & $120.6(4)$ \\
\hline
\end{tabular}




$\begin{array}{llllllll}\text { C18 } & \text { P1 } & \text { C24 } & 106.02(15) & \text { C21 } & \text { C20 } & \text { C19 } & 119.6(4) \\ \text { C18 } & \text { P1 } & \text { C12 } & 103.77(13) & \text { C15 } & \text { C16 } & \text { C17 } & 120.1(3) \\ \text { C12 } & \text { P1 } & \text { Pd1 } & 113.88(10) & \text { C22 } & \text { C21 } & \text { C20 } & 120.4(4) \\ \text { C12 } & \text { P1 } & \text { C24 } & 103.28(13) & \text { C27 } & \text { C26 } & \text { C25 } & 120.0(4) \\ \text { C29 } & \text { C24 } & \text { P1 } & 120.8(2) & \text { C27 } & \text { C28 } & \text { C29 } & 119.7(4) \\ \text { C25 } & \text { C24 } & \text { P1 } & 120.0(3) & \text { C28 } & \text { C27 } & \text { C26 } & 120.3(4) \\ \text { C25 } & \text { C24 } & \text { C29 } & 118.2(3) & \text { C11 } & \text { C6 } & \text { C7 } & 119.3(5) \\ \text { C23 } & \text { C18 } & \text { P1 } & 117.2(3) & \text { C5 } & \text { C6 } & \text { C11 } & 119.5(4) \\ \text { C19 } & \text { C18 } & \text { P1 } & 123.9(3) & \text { C5 } & \text { C6 } & \text { C7 } & 121.3(4) \\ \text { C19 } & \text { C18 } & \text { C23 } & 118.8(3) & \text { C9 } & \text { C10 } & \text { C11 } & 121.1(4) \\ \text { C28 } & \text { C29 } & \text { C24 } & 121.2(3) & \text { C8 } & \text { C9 } & \text { C10 } & 119.7(5) \\ \text { C24 } & \text { C25 } & \text { C26 } & 120.5(3) & \text { C8 } & \text { C7 } & \text { C6 } & 119.5(5) \\ \text { C12 } & \text { C13 } & \text { C14 } & 120.4(3) & \text { C2 } & \text { C3 } & \text { Pd1 } & 65.49(19) \\ \text { C18 } & \text { C23 } & \text { C22 } & 119.9(4) & \text { C2 } & \text { C3 } & \text { C4 } & 120.0(4) \\ \text { C13 } & \text { C12 } & \text { P1 } & 122.8(2) & \text { C4 } & \text { C3 } & \text { Pd1 } & 125.3(3) \\ \text { C13 } & \text { C12 } & \text { C17 } & 118.5(3) & \text { C21 } & \text { C22 } & \text { C23 } & 120.6(4) \\ \text { C17 } & \text { C12 } & \text { P1 } & 118.6(2) & \text { C7 } & \text { C8 } & \text { C9 } & 122.1(5) \\ \text { C16 } & \text { C17 } & \text { C12 } & 120.9(3) & \text { C4 } & \text { C5 } & \text { C6 } & 122.4(4) \\ \text { C1 } & \text { C2 } & \text { Pd1 } & 63.79(17) & \text { C5 } & \text { C4 } & \text { C3 } & 119.8(5)\end{array}$

Table 6 Torsion Angles for XYN09-Cl-111.

$\begin{array}{llllllllll}\text { A } & \mathbf{B} & \mathbf{C} & \mathbf{D} & \text { Angle }^{\circ} & \mathbf{A} & \mathbf{B} & \mathbf{C} & \mathbf{D} & \text { Angle }^{\circ} \\ \mathrm{Pd} 1 & \mathrm{P} 1 & \mathrm{C} 24 & \mathrm{C} 25 & 67.6(3) & \mathrm{C} 12 & \mathrm{P} 1 & \mathrm{C} 18 & \mathrm{C} 23 & -78.5(3) \\ \mathrm{Pd} 1 & \mathrm{P} 1 & \mathrm{C} 18 & \mathrm{C} 23 & 47.9(3) & \mathrm{C} 12 & \mathrm{P} 1 & \mathrm{C} 18 & \mathrm{C} 19 & 100.1(3) \\ \mathrm{Pd} 1 & \mathrm{P} 1 & \mathrm{C} 18 & \mathrm{C} 19 & -133.5(3) & \mathrm{C} 12 & \mathrm{C} 13 & \mathrm{C} 14 & \mathrm{C} 15 & 0.0(6) \\ \mathrm{Pd} 1 & \mathrm{P} 1 & \mathrm{C} 12 & \mathrm{C} 13 & -150.1(2) & \mathrm{C} 12 & \mathrm{C} 17 & \mathrm{C} 16 & \mathrm{C} 15 & 1.7(6) \\ \mathrm{Pd} 1 & \mathrm{P} 1 & \mathrm{C} 12 & \mathrm{C} 17 & 34.6(3) & \mathrm{C} 2 & \mathrm{C} 11 & \mathrm{C} 6 & \mathrm{C} 7 & -178.7(3) \\ \mathrm{Pd} 1 & \mathrm{C} 2 & \mathrm{C} 11 & \mathrm{C} 6 & -96.9(3) & \mathrm{C} 2 & \mathrm{C} 11 & \mathrm{C} 6 & \mathrm{C} 5 & 1.0(5) \\ \mathrm{Pd} 1 & \mathrm{C} 2 & \mathrm{C} 11 & \mathrm{C} 10 & 84.0(4) & \mathrm{C} 2 & \mathrm{C} 11 & \mathrm{C} 10 & \mathrm{C} 9 & 179.5(3) \\ \mathrm{Pd} 1 & \mathrm{C} 2 & \mathrm{C} 3 & \mathrm{C} 4 & 118.0(4) & \mathrm{C} 2 & \mathrm{C} 3 & \mathrm{C} 4 & \mathrm{C} 5 & 0.7(7) \\ \mathrm{Pd} 1 & \mathrm{C} 3 & \mathrm{C} 4 & \mathrm{C} 5 & 80.5(6) & \mathrm{C} 1 & \mathrm{C} 2 & \mathrm{C} 11 & \mathrm{C} 6 & -172.3(3) \\ \mathrm{P} 1 & \mathrm{C} 24 & \mathrm{C} 29 & \mathrm{C} 28 & 166.9(3) & \mathrm{C} 1 & \mathrm{C} 2 & \mathrm{C} 11 & \mathrm{C} 10 & 8.6(5) \\ \mathrm{P} 1 & \mathrm{C} 24 & \mathrm{C} 25 & \mathrm{C} 26 & -168.2(3) & \mathrm{C} 1 & \mathrm{C} 2 & \mathrm{C} 3 & \mathrm{P} 1 & 54.3(2) \\ \mathrm{P} 1 & \mathrm{C} 18 & \mathrm{C} 23 & \mathrm{C} 22 & 176.7(3) & \mathrm{C} 1 & \mathrm{C} 2 & \mathrm{C} 3 & \mathrm{C} 4 & 172.3(4) \\ \mathrm{P} 1 & \mathrm{C} 18 & \mathrm{C} 19 & \mathrm{C} 20 & -177.4(3) & \mathrm{C} 11 & \mathrm{C} 2 & \mathrm{C} 1 & \mathrm{P} 1 & 106.4(3) \\ \mathrm{P} 1 & \mathrm{C} 12 & \mathrm{C} 17 & \mathrm{C} 16 & 174.3(3) & \mathrm{C} 11 & \mathrm{C} 2 & \mathrm{C} 3 & \mathrm{P} 1 & -115.8(3) \\ \mathrm{C} 24 & \mathrm{P} 1 & \mathrm{C} 18 & \mathrm{C} 23 & 173.1(2) & \mathrm{C} 11 & \mathrm{C} 2 & \mathrm{C} 3 & \mathrm{C} 4 & 2.2(5) \\ \mathrm{C} 24 & \mathrm{P} 1 & \mathrm{C} 18 & \mathrm{C} 19 & -8.3(3) & \mathrm{C} 11 & \mathrm{C} 6 & \mathrm{C} 7 & \mathrm{C} 8 & -0.9(6) \\ \mathrm{C} 24 & \mathrm{P} 1 & \mathrm{C} 12 & \mathrm{C} 13 & 88.7(3) & \mathrm{C} 11 & \mathrm{C} 6 & \mathrm{C} 5 & \mathrm{C} 4 & 2.0(7) \\ \mathrm{C} 24 & \mathrm{P} 1 & \mathrm{C} 12 & \mathrm{C} 17 & -86.5(3) & \mathrm{C} 11 & \mathrm{C} 10 & \mathrm{C} 9 & \mathrm{C} 8 & -0.7(6) \\ \mathrm{C} 24 & \mathrm{C} 29 & \mathrm{C} 28 & \mathrm{C} 27 & 1.4(6) & \mathrm{C} 14 & \mathrm{C} 13 & \mathrm{C} 12 & \mathrm{P} 1 & -175.0(3) \\ \mathrm{C} 24 & \mathrm{C} 25 & \mathrm{C} 26 & \mathrm{C} 27 & 1.1(6) & \mathrm{C} 14 & \mathrm{C} 13 & \mathrm{C} 12 & \mathrm{C} 17 & 0.3(5) \\ \mathrm{C} 18 & \mathrm{P} 1 & \mathrm{C} 24 & \mathrm{C} 29 & 130.6(3) & \mathrm{C} 14 & \mathrm{C} 15 & \mathrm{C} 16 & \mathrm{C} 17 & -1.4(6)\end{array}$




$\begin{array}{llllllllll}\text { C18 } & \text { P1 } & \text { C24 } & \text { C25 } & -60.9(3) & \text { C19 } & \text { C18 } & \text { C23 } & \text { C22 } & -2.0(5) \\ \text { C18 } & \text { P1 } & \text { C12 } & \text { C13 } & -21.8(3) & \text { C19 } & \text { C20 } & \text { C21 } & \text { C22 } & -0.6(7) \\ \text { C18 } & \text { P1 } & \text { C12 } & \text { C17 } & 163.0(3) & \text { C20 } & \text { C21 } & \text { C22 } & \text { C23 } & -0.3(6) \\ \text { C18 } & \text { C23 } & \text { C22 } & \text { C21 } & 1.6(6) & \text { C6 } & \text { C11 } & \text { C10 } & \text { C9 } & 0.4(5) \\ \text { C18 } & \text { C19 } & \text { C20 } & \text { C21 } & 0.1(6) & \text { C6 } & \text { C7 } & \text { C8 } & \text { C9 } & 0.6(7) \\ \text { C29 } & \text { C24 } & \text { C25 } & \text { C26 } & 0.5(5) & \text { C6 } & \text { C5 } & \text { C4 } & \text { C3 } & -2.9(8) \\ \text { C29 } & \text { C28 } & \text { C27 } & \text { C26 } & 0.3(6) & \text { C10 } & \text { C11 } & \text { C6 } & \text { C7 } & 0.4(5) \\ \text { C25 } & \text { C24 } & \text { C29 } & \text { C28 } & -1.8(5) & \text { C10 } & \text { C11 } & \text { C6 } & \text { C5 } & -179.9(4) \\ \text { C25 } & \text { C26 } & \text { C27 } & \text { C28 } & -1.6(6) & \text { C10 } & \text { C9 } & \text { C8 } & \text { C7 } & 0.2(7) \\ \text { C13 } & \text { C12 } & \text { C17 } & \text { C16 } & -1.1(5) & \text { C7 } & \text { C6 } & \text { C5 } & \text { C4 } & -178.2(5) \\ \text { C13 } & \text { C14 } & \text { C15 } & \text { C16 } & 0.6(6) & \text { C3 } & \text { C2 } & \text { C1 } & \text { Pd1 } & -63.1(3) \\ \text { C23 } & \text { C18 } & \text { C19 } & \text { C20 } & 1.2(5) & \text { C3 } & \text { C2 } & \text { C11 } & \text { C6 } & -3.1(5) \\ \text { C12 } & \text { P1 } & \text { C24 } & \text { C29 } & 21.8(3) & \text { C3 } & \text { C2 } & \text { C11 } & \text { C10 } & 177.8(3) \\ \text { C12 } & \text { P1 } & \text { C24 } & \text { C25 } & -169.7(3) & \text { C5 } & \text { C6 } & \text { C7 } & \text { C8 } & 179.3(5)\end{array}$

Table 7 Hydrogen Atom Coordinates $\left(\AA \AA^{\times} \times 10^{4}\right)$ and Isotropic Displacement Parameters $\left(\AA 2 \times 10^{3}\right)$ for XYN09-Cl-111.

$\begin{array}{lrrrl}\text { Atom } & \mathbf{x} & \mathbf{y} & \mathbf{z} & \mathbf{U}(\mathbf{e q}) \\ \text { H29 } & 4669 & 6525 & 4210 & 61 \\ \text { H25 } & 9090 & 6700 & 4351 & 65 \\ \text { H13 } & 3685 & 7303 & 1151 & 61 \\ \text { H23 } & 5901 & 6788 & -557 & 69 \\ \text { H17 } & 3405 & 5916 & 1752 & 58 \\ \text { H1A } & 7870 & 5745 & 4303 & 6 \\ \text { H1B } & 6448 & 5361 & 3644 & 6 \\ \text { H14 } & 976 & 7372 & 605 & 72 \\ \text { H15 } & -516 & 6717 & 636 & 7 \\ \text { H19 } & 7521 & 7447 & 3171 & 77 \\ \text { H20 } & 8155 & 8107 & 2144 & 100 \\ \text { H16 } & 716 & 5993 & 1262 & 74 \\ \text { H21 } & 7612 & 8103 & -222 & 98 \\ \text { H26 } & 10117 & 6665 & 6793 & 83 \\ \text { H28 } & 5715 & 6463 & 6640 & 83 \\ \text { H27 } & 8431 & 6529 & 7921 & 89 \\ \text { H10 } & 10377 & 5771 & 5127 & 76 \\ \text { H9 } & 13115 & 5850 & 5864 & 101 \\ \text { H7 } & 13103 & 4963 & 2876 & 106 \\ \text { H3 } & 6379 & 4951 & 1485 & 83 \\ \text { H22 } & 6485 & 7455 & -1566 & 93 \\ \text { H8 } & 14431 & 5444 & 4733 & 117 \\ \text { H5 } & 10547 & 4620 & 1232 & 118 \\ \text { H4 } & 7837 & 4561 & 348 & 118\end{array}$




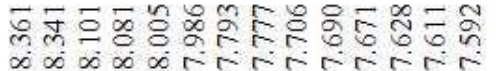

ڤั

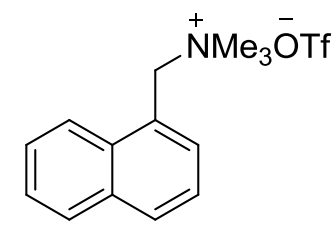

1a

${ }^{1} \mathrm{H}$ NMR $\left(400 \mathrm{MHz}, \mathrm{CD}_{3} \mathrm{OD}\right)$

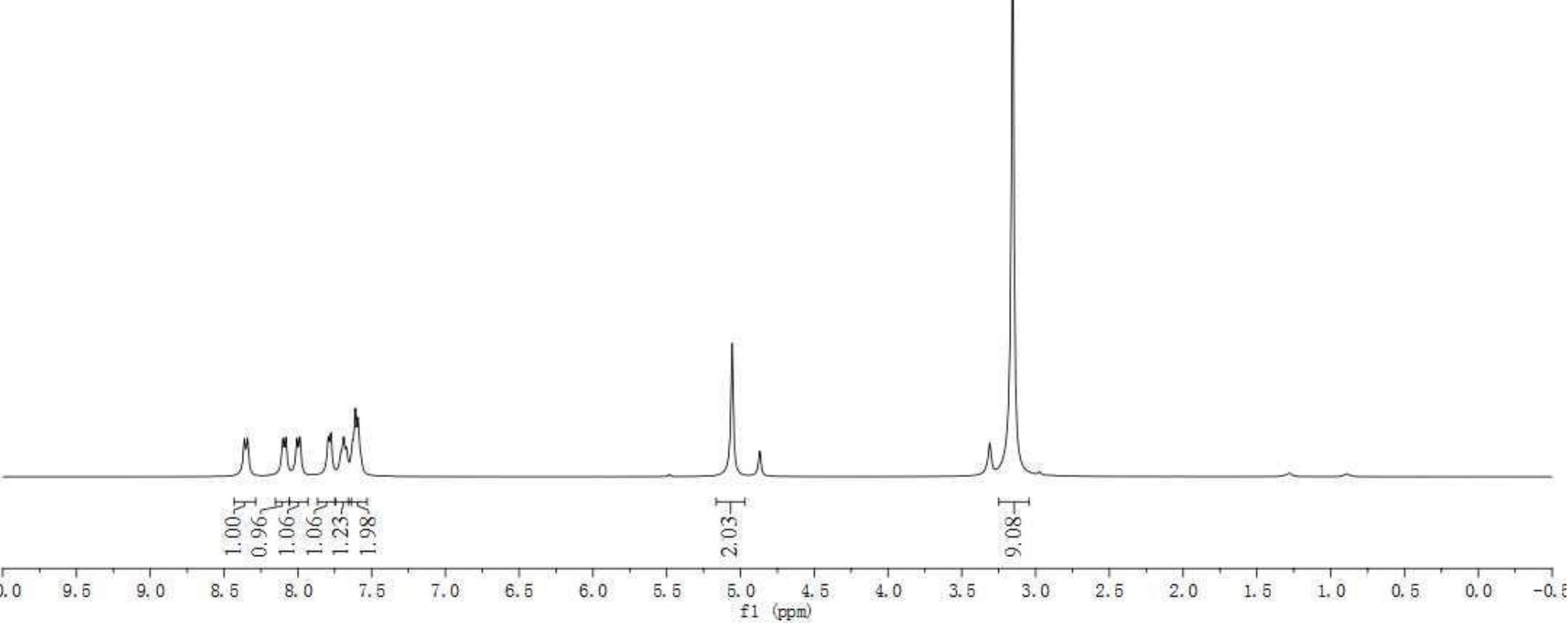




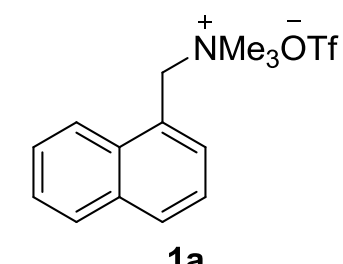

${ }^{13} \mathrm{C}$ NMR (100 MHz, $\left.\mathrm{CD}_{3} \mathrm{OD}\right)$

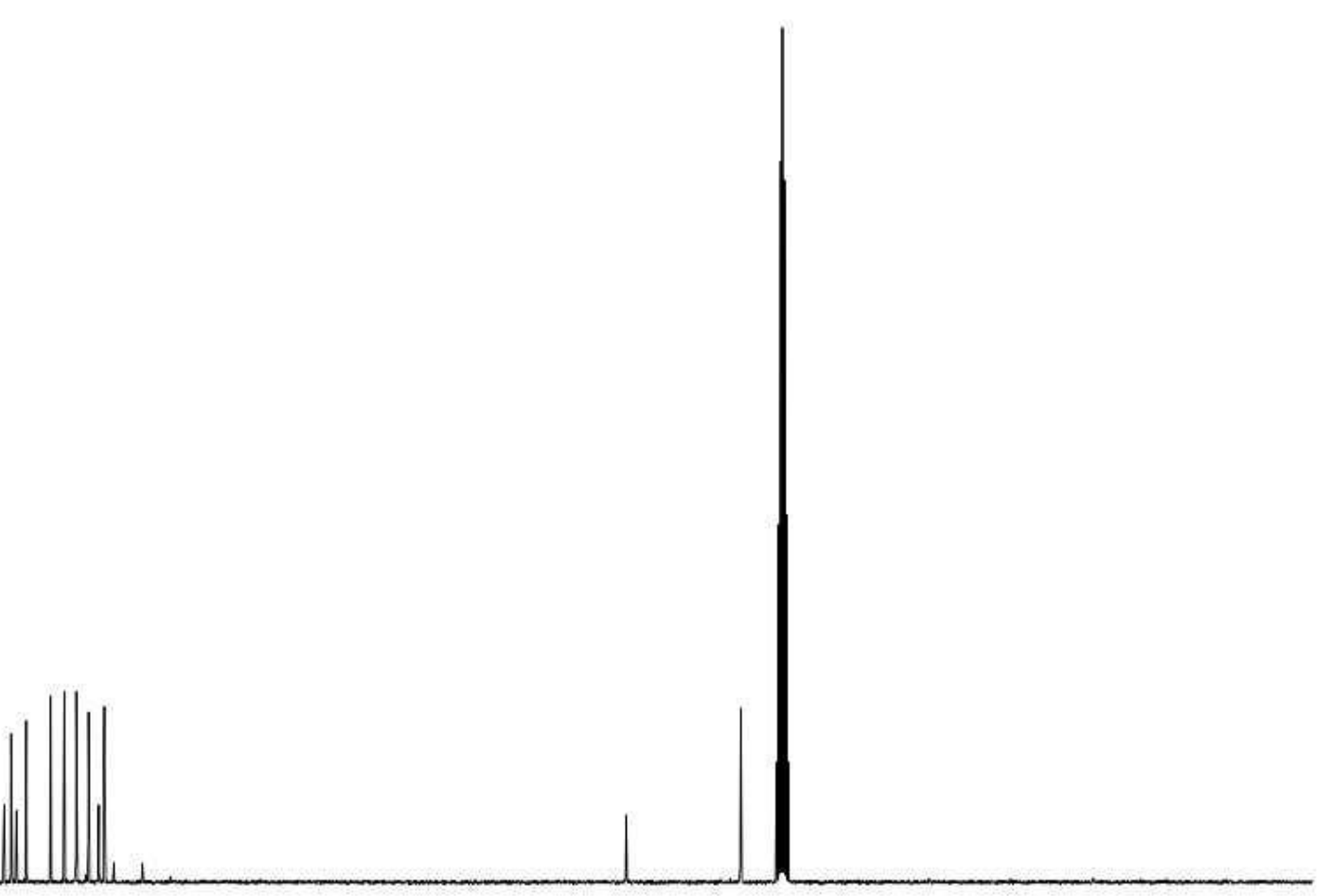

$200 \quad 190 \quad 180$

$\begin{array}{rrr}140 & 130 & 120\end{array}$

110

${ }_{\mathrm{f} 1}^{100} \mathrm{(ppm)}$ 


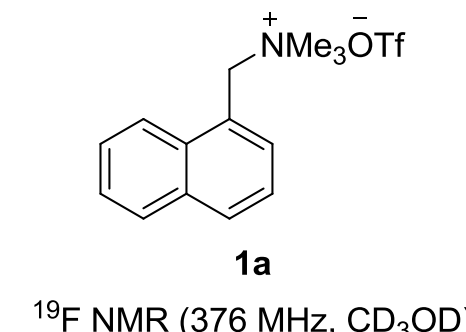

${ }^{19} \mathrm{~F} \mathrm{NMR}\left(376 \mathrm{MHz}, \mathrm{CD}_{3} \mathrm{OD}\right)$ 


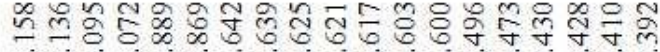

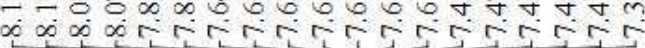

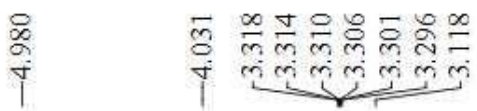

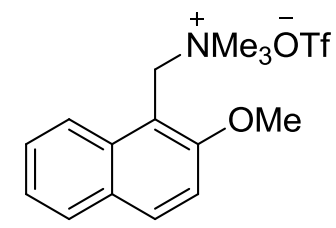

1b

${ }^{1} \mathrm{H}$ NMR $\left(400 \mathrm{MHz}, \mathrm{CD}_{3} \mathrm{OD}\right)$

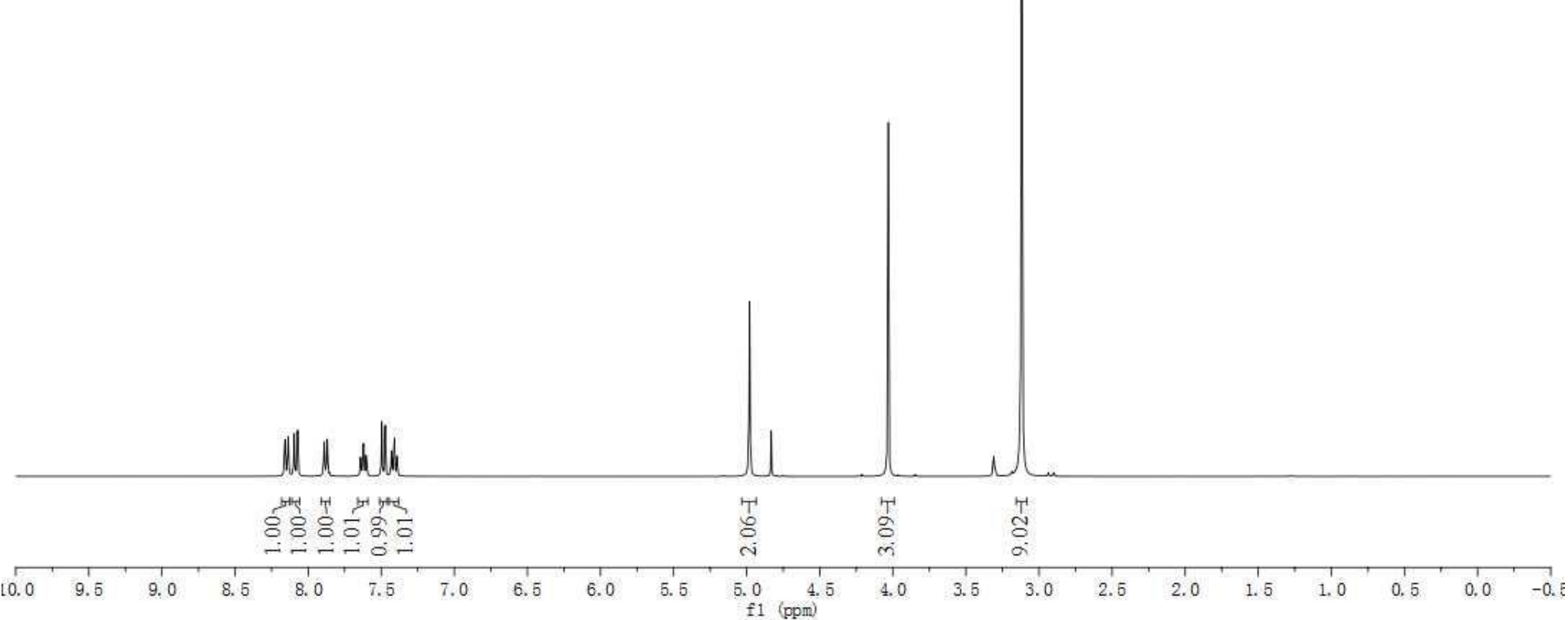




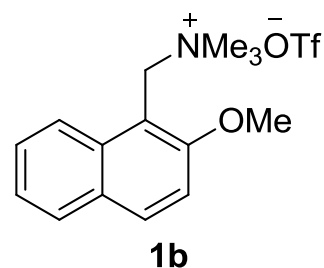

${ }^{13} \mathrm{C}$ NMR (100 MHz, $\left.\mathrm{CD}_{3} \mathrm{OD}\right)$

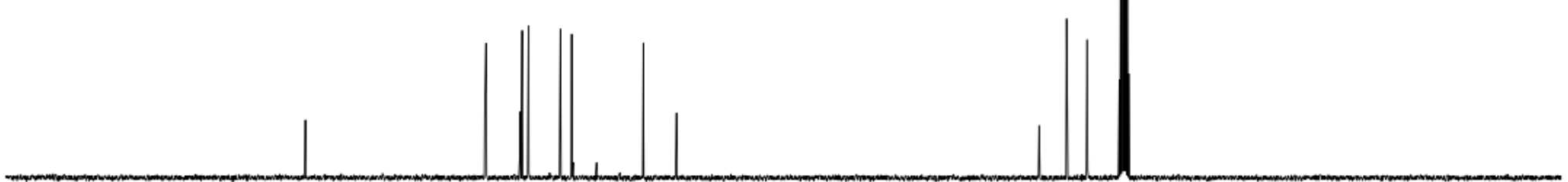

$\begin{array}{llllllllllll}200 & 190 & 180 & 170 & 160 & 150 & 140 & 130 & 120 & 110 & 100 & 90\end{array}$ 


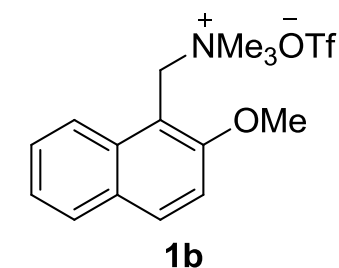

${ }^{19} \mathrm{~F}$ NMR (376 MHz, CD 3 OD)

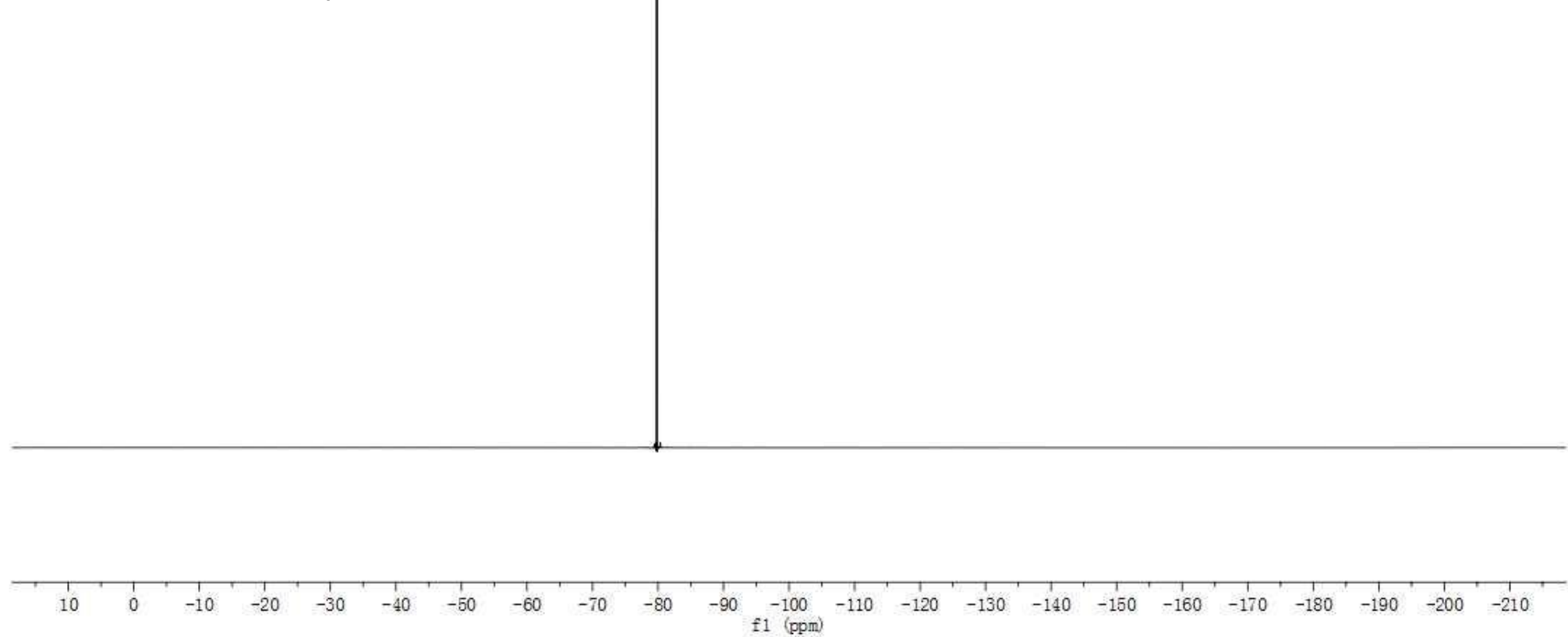




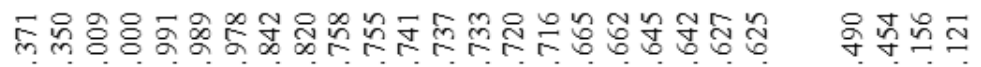

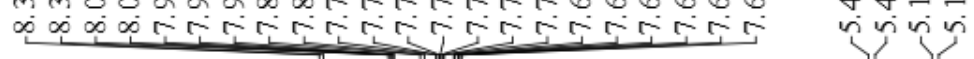

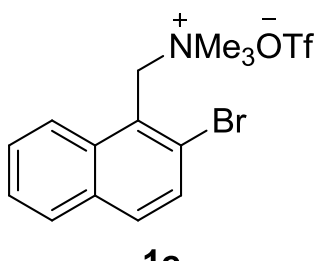

$1 c$

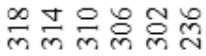

लmingm

${ }^{1} \mathrm{H}$ NMR $\left(400 \mathrm{MHz}, \mathrm{CD}_{3} \mathrm{OD}\right)$

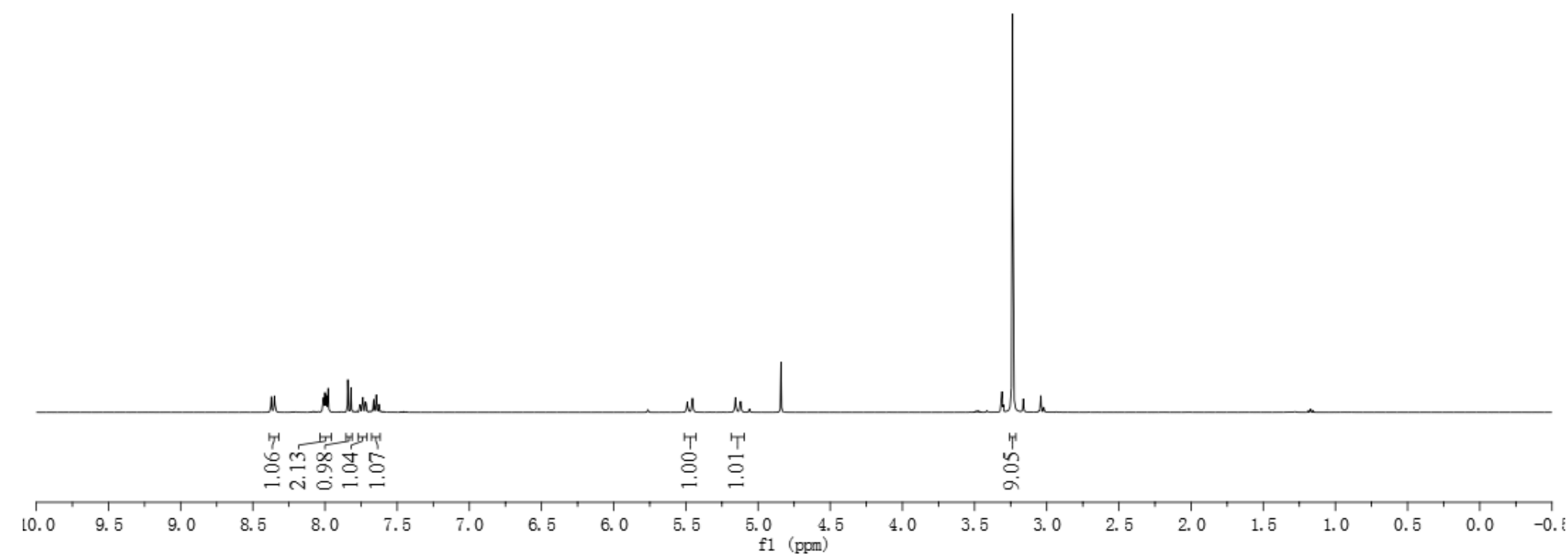




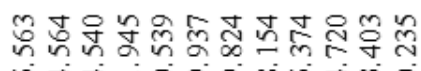

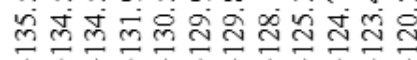

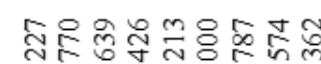

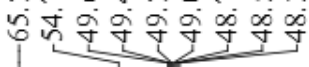

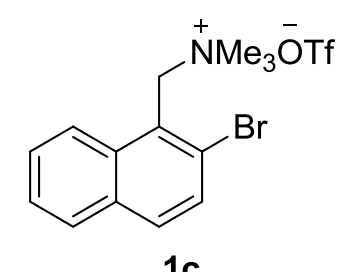

${ }^{13} \mathrm{C} \mathrm{NMR}\left(100 \mathrm{MHz}, \mathrm{CD}_{3} \mathrm{OD}\right)$

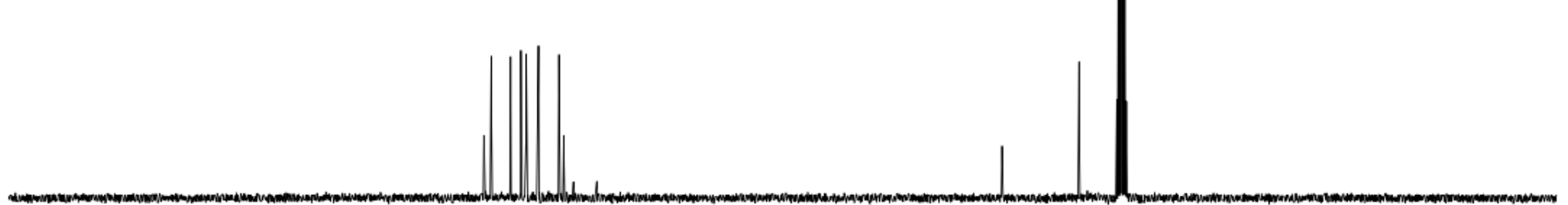

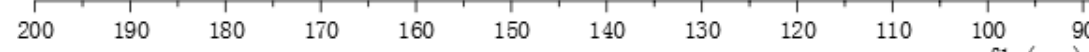




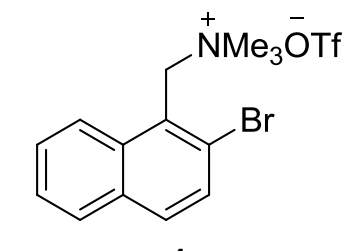

1c

${ }^{19} \mathrm{~F}$ NMR (376 MHz, CD 3 OD)

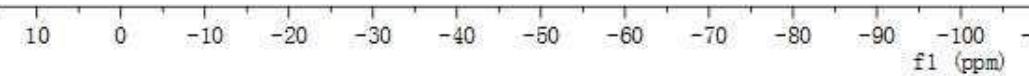




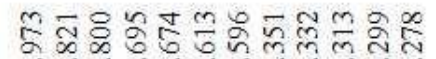

rising

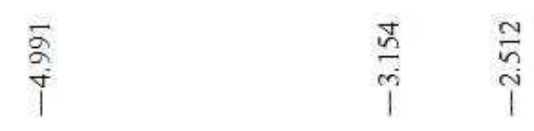

$\stackrel{8}{8}$

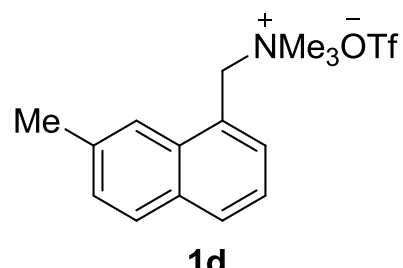

${ }^{1} \mathrm{H}$ NMR $\left(400 \mathrm{MHz}, \mathrm{CDCl}_{3}\right)$

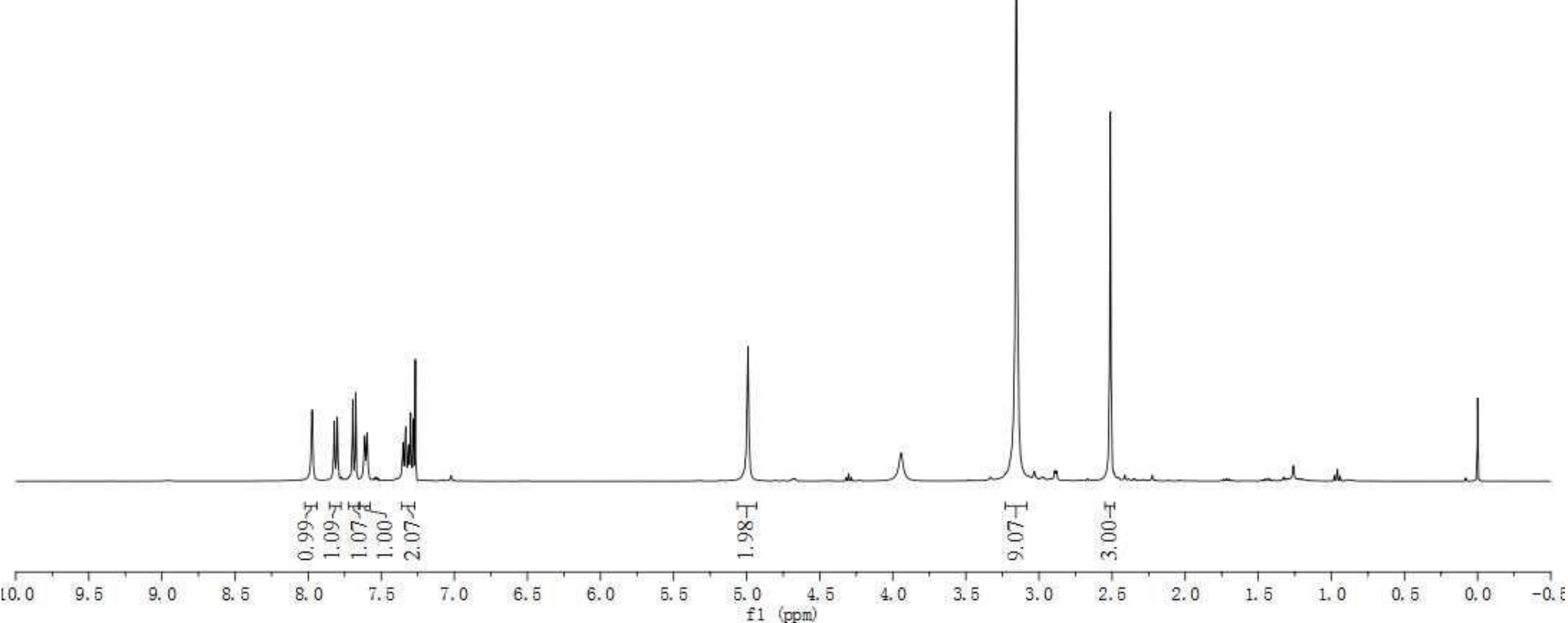




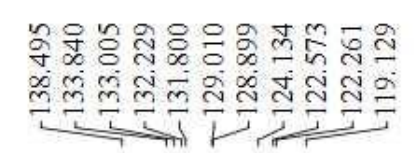

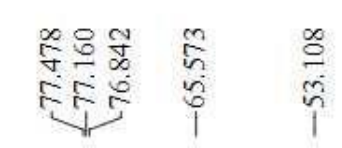

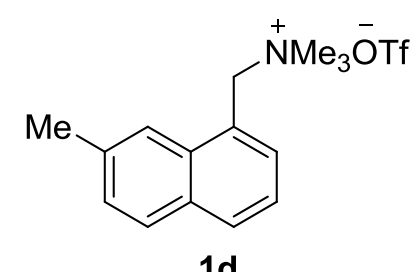

${ }^{13} \mathrm{C}$ NMR (100 MHz, $\mathrm{CDCl}_{3}$ )

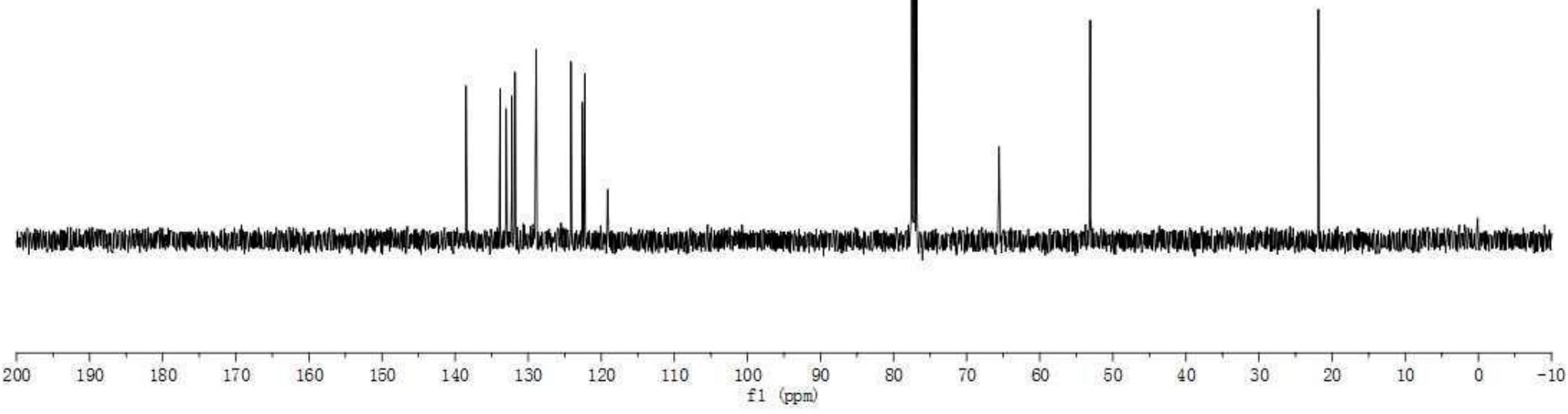




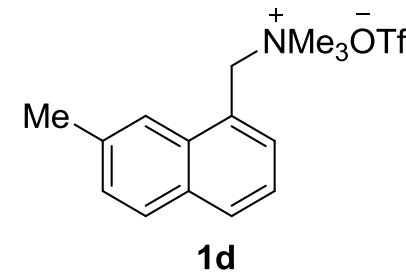

${ }^{19} \mathrm{~F}$ NMR $\left(376 \mathrm{MHz}, \mathrm{CDCl}_{3}\right)$

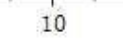

$-10$

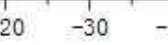

$-40 \quad-50$

$-60 \quad-70$

$-80$

$-100$ 


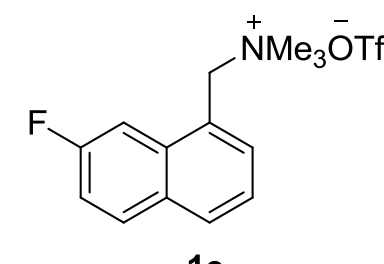

${ }^{1} \mathrm{H}$ NMR $\left(400 \mathrm{MHz}, \mathrm{CD}_{3} \mathrm{OD}\right)$

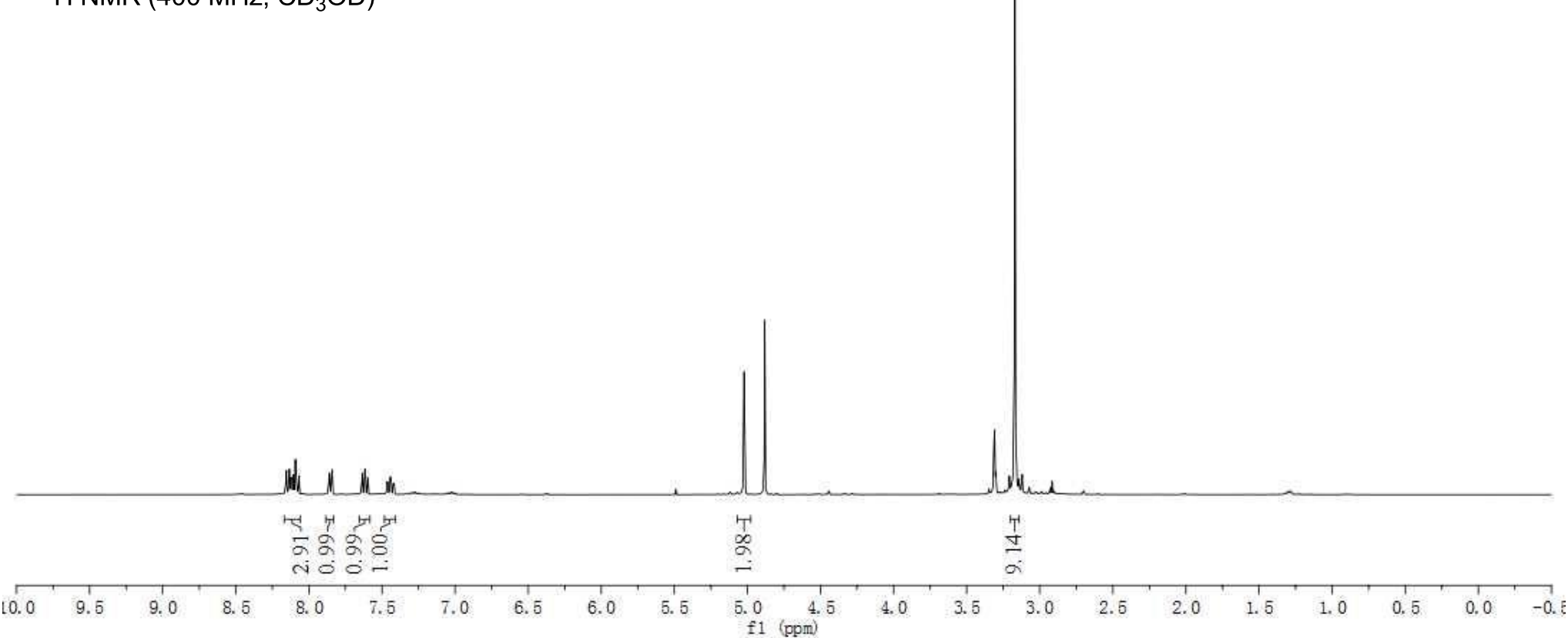




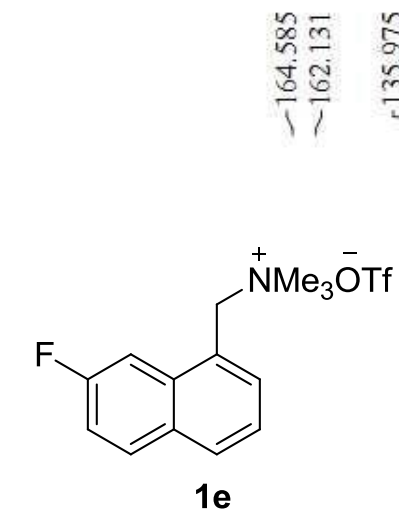

${ }^{13} \mathrm{C}$ NMR (100 MHz, CD $\left.{ }_{3} \mathrm{OD}\right)$

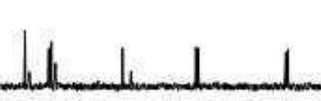

$200 \quad 190 \quad 1,1$

${ }_{140}^{1}+130 \quad 1$

${ }_{\mathrm{fl}}^{100}(\mathrm{ppm})$ 


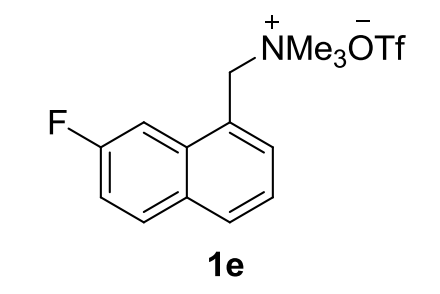

${ }^{19} \mathrm{~F}$ NMR (376 MHz, CD 3 OD)

\author{
(
}




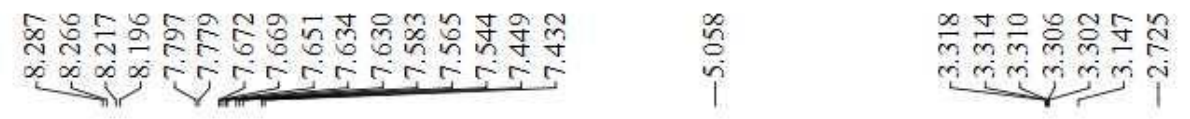

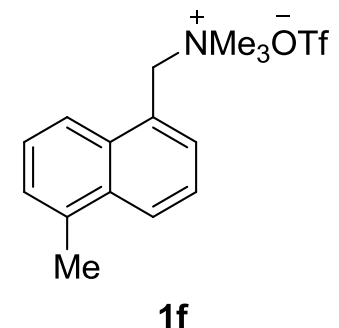

${ }^{1} \mathrm{H}$ NMR (400 MHz, CD ${ }_{3} \mathrm{OD}$ )

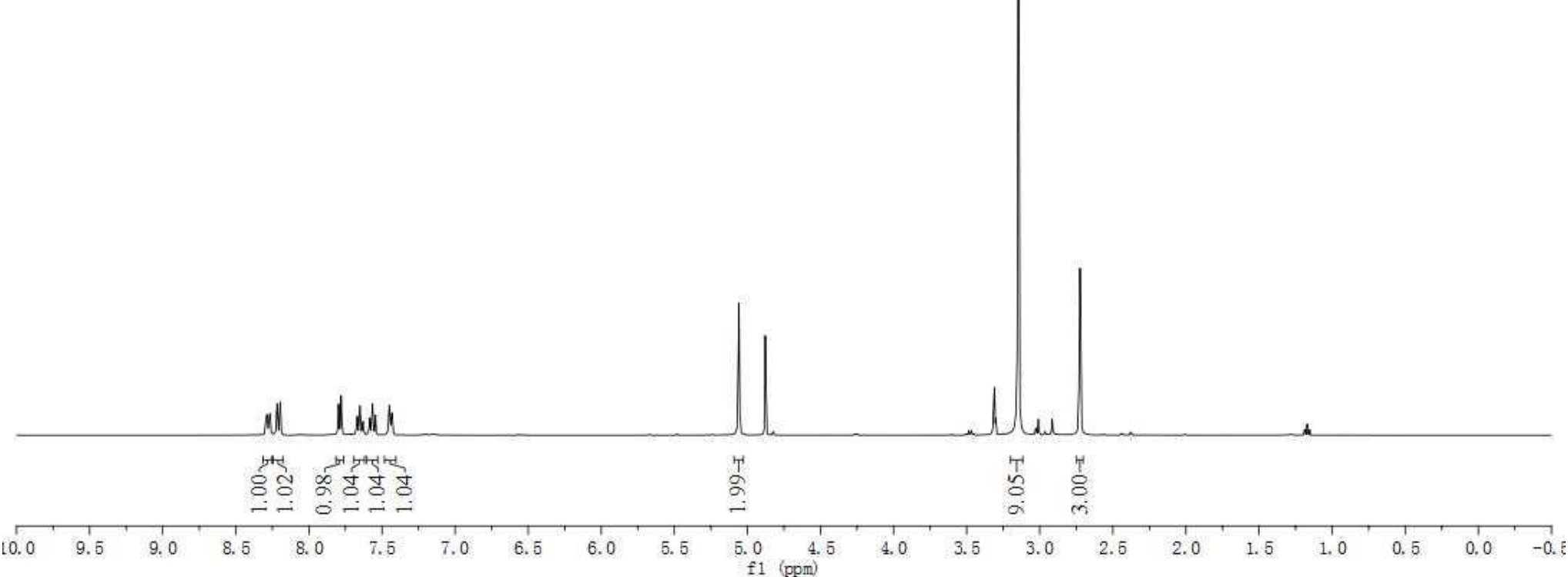




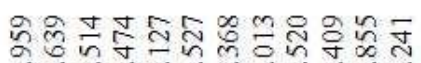

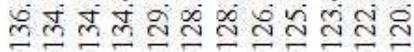 \\ -1 $-7 \div \div \div$}

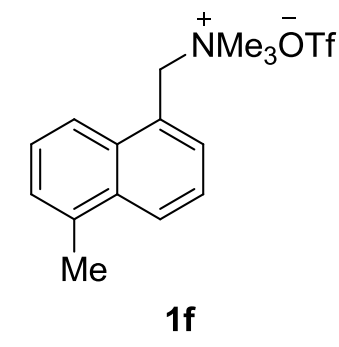

${ }^{13} \mathrm{C}$ NMR $\left(100 \mathrm{MHz}, \mathrm{CD}_{3} \mathrm{OD}\right)$

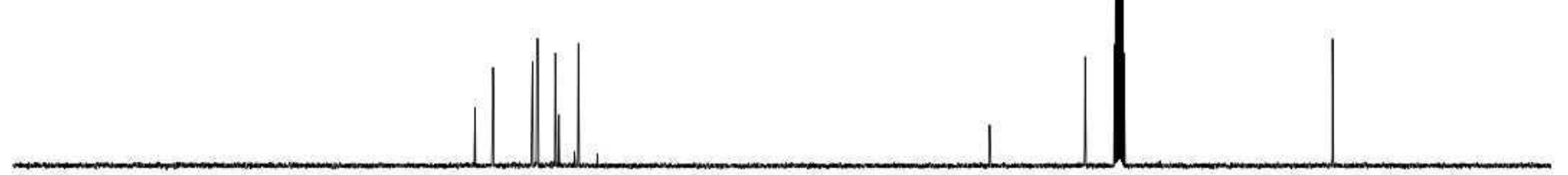

$200 \quad 190 \quad 180$

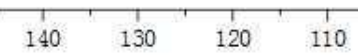

$100 \quad 90$

80

1 60

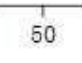




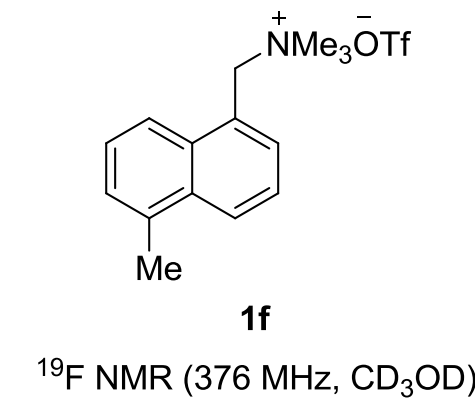

F NMR (376 MHz, $\left.\mathrm{CD}_{3} \mathrm{OD}\right)$

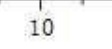

$-20 \quad-30$

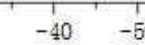

$-60 \quad-70$

$\begin{array}{lllllllllllllll}-90 & -100 & -110 & -120 & -130 & -140 & -150 & -160 & -170 & -180 & -190 & -200 & -210\end{array}$ 


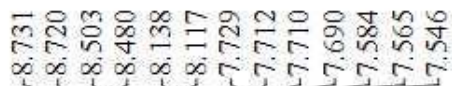
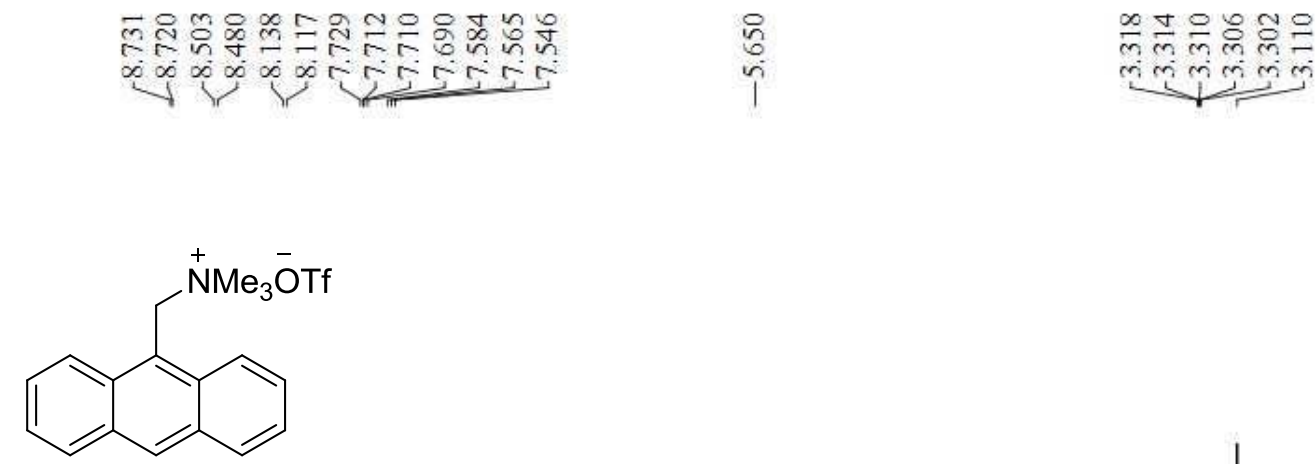

$1 \mathrm{~g}$

${ }^{1} \mathrm{H}$ NMR $\left(400 \mathrm{MHz}, \mathrm{CD}_{3} \mathrm{OD}\right)$

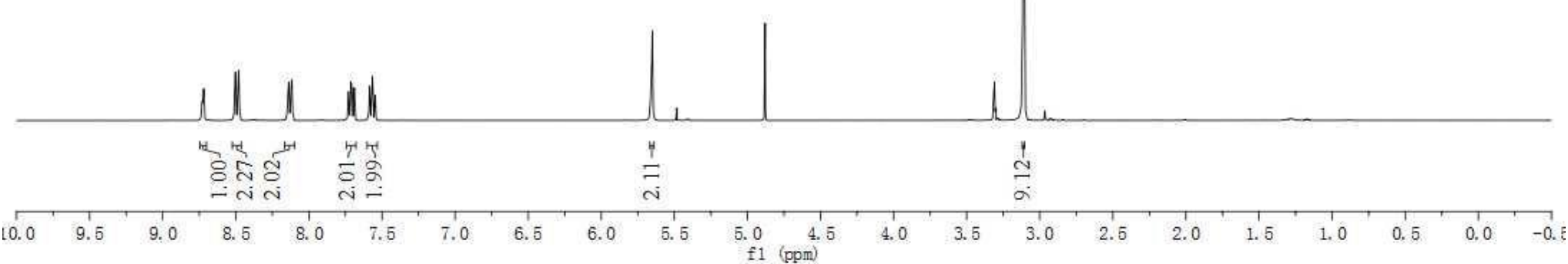




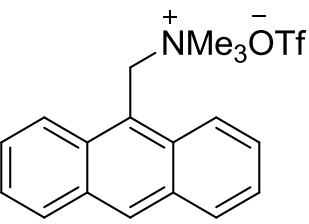

$1 \mathrm{~g}$

${ }^{13} \mathrm{C}$ NMR (100 MHz, $\left.\mathrm{CD}_{3} \mathrm{OD}\right)$

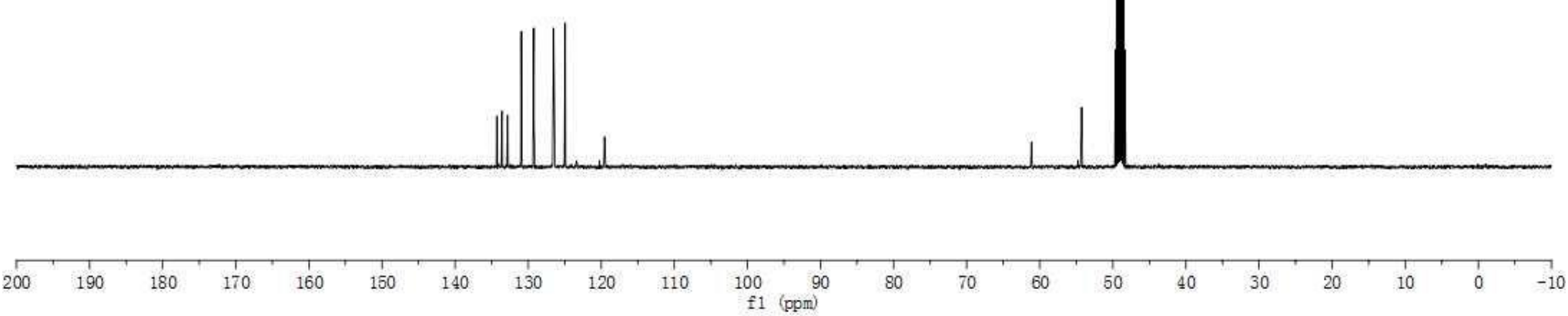




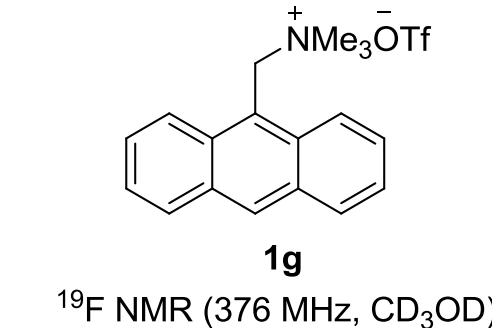

10

$-10$

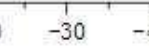

$-40 \quad-50$

$\begin{array}{ll}-60 & -70\end{array}$

$-90 \quad-100$ 


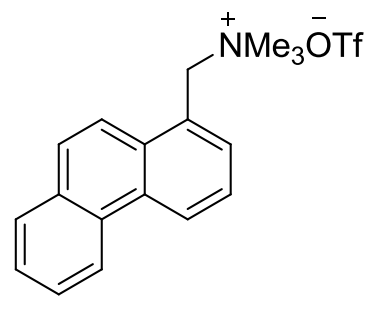

1h

${ }^{1} \mathrm{H}$ NMR (400 MHz, $\mathrm{CD}_{3} \mathrm{OD}$ )

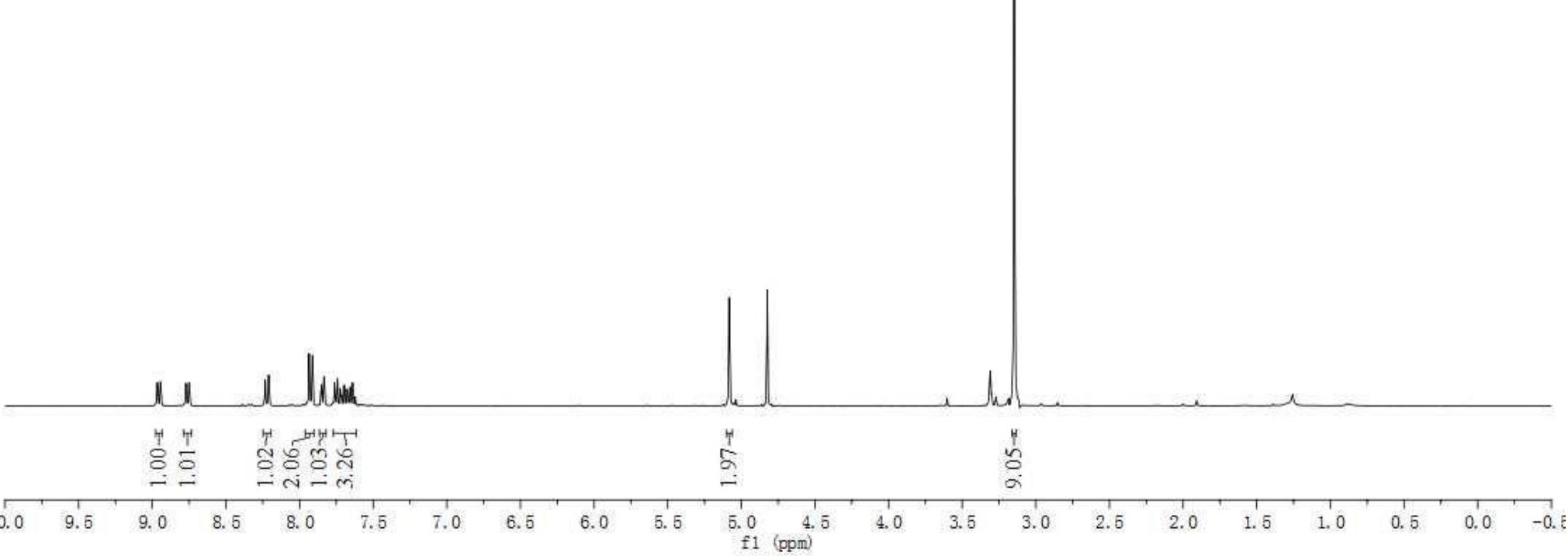




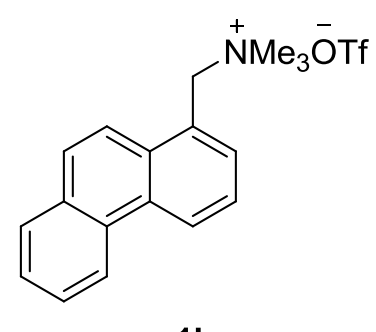

$1 \mathrm{~h}$

${ }^{13} \mathrm{C}$ NMR $\left(100 \mathrm{MHz}, \mathrm{CD}_{3} \mathrm{OD}\right)$

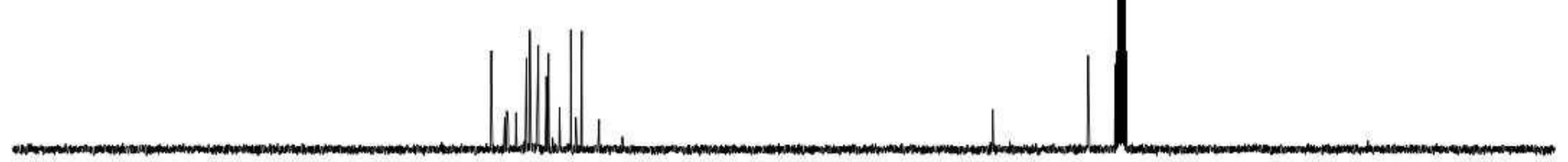

\begin{tabular}{llllllllllll}
\hline 200 & 190 & 180 & 170 & 160 & 150 & 140 & 130 & 120 & 110 & 100
\end{tabular} 


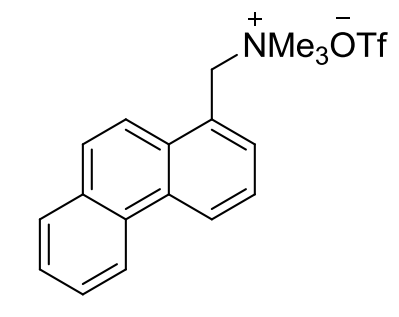

1h

${ }^{19} \mathrm{~F}$ NMR (376 MHz, CD ${ }_{3} \mathrm{OD}$ )

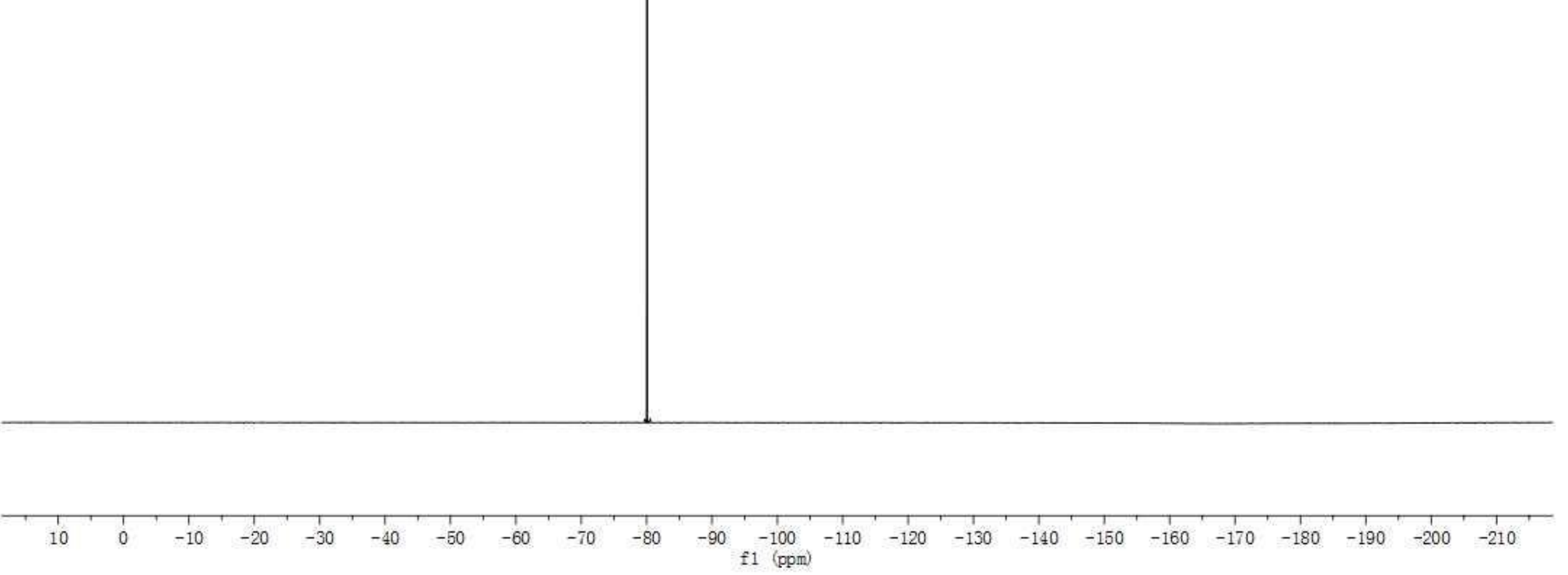



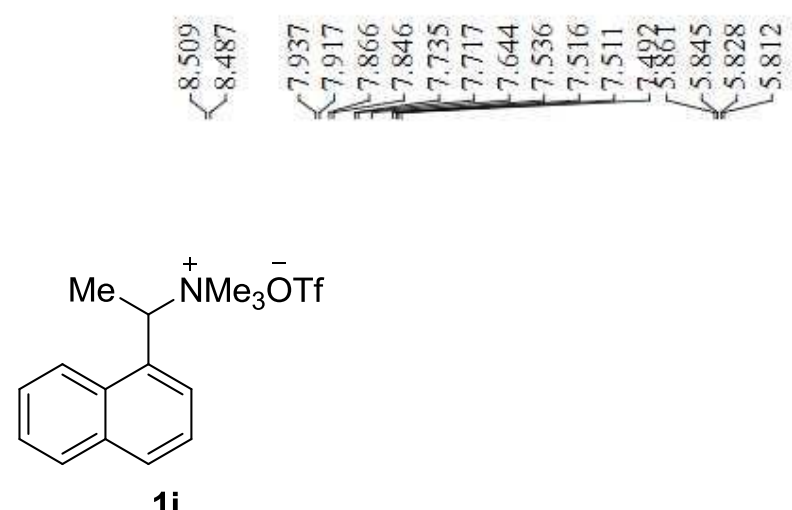

${ }^{1} \mathrm{H}$ NMR $\left(400 \mathrm{MHz}, \mathrm{CDCl}_{3}\right)$

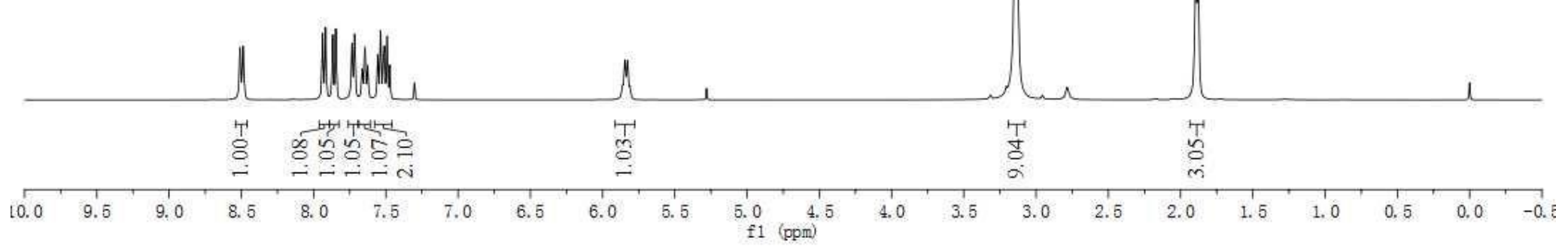



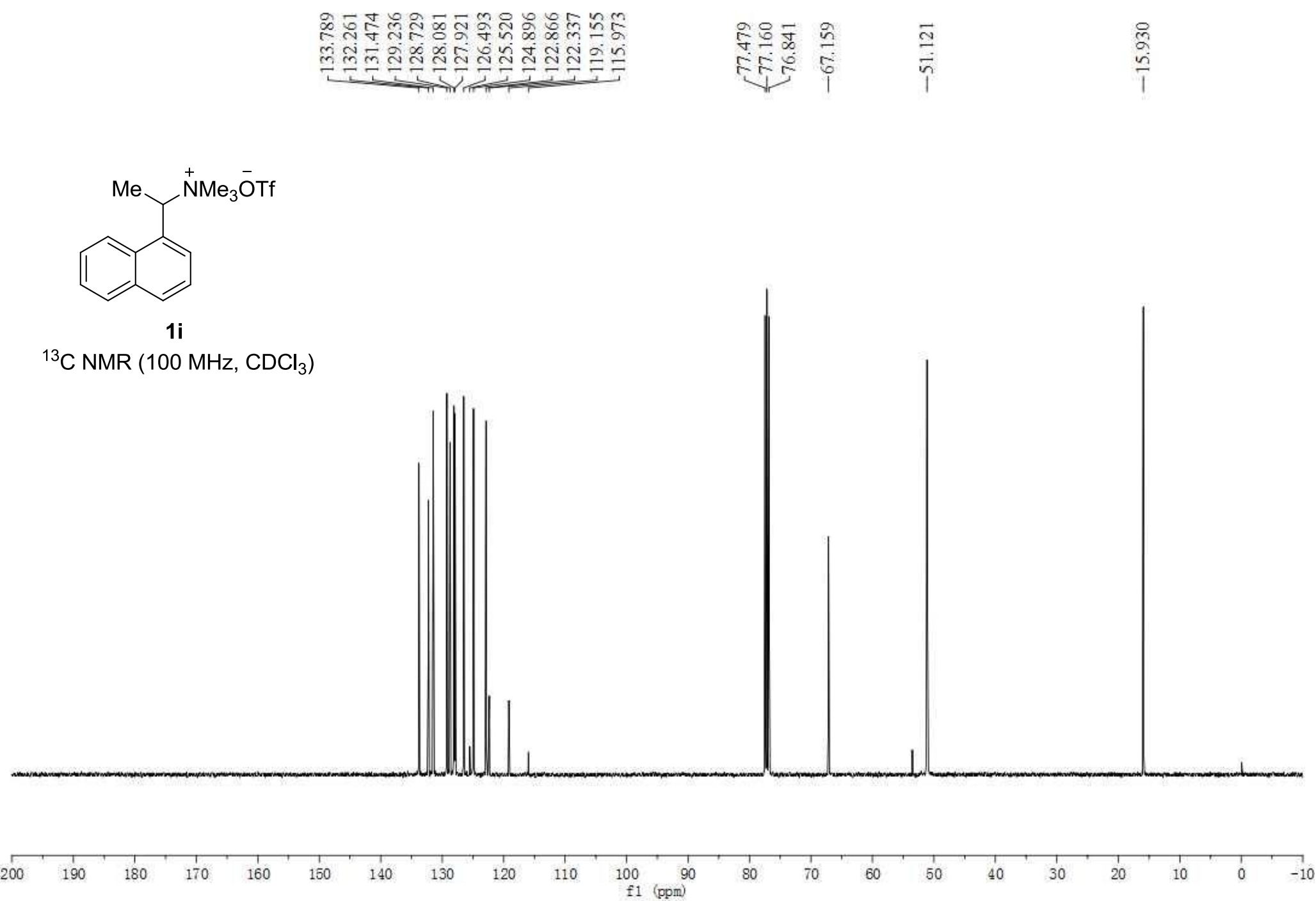


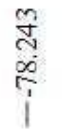

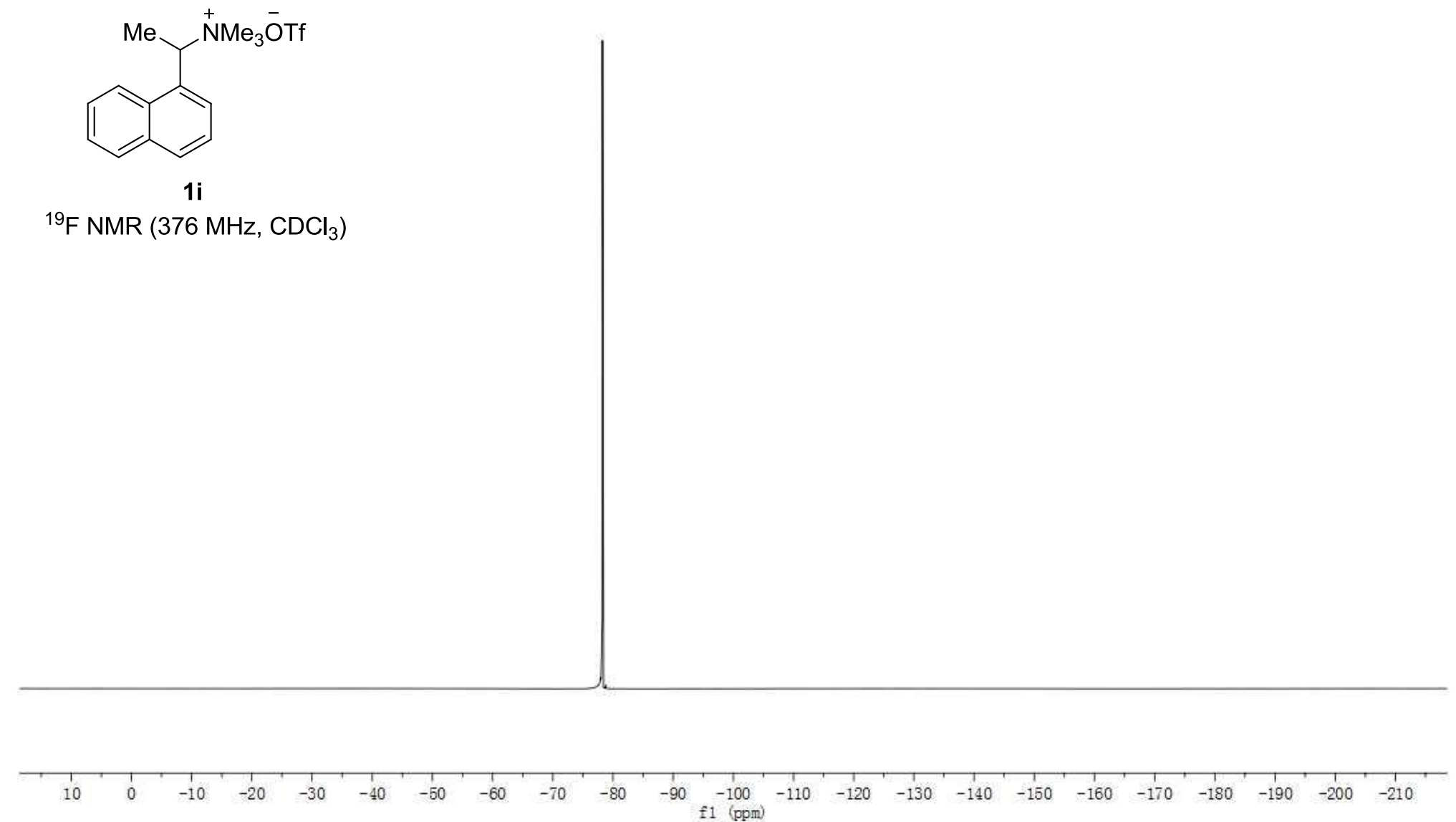




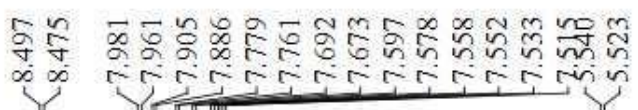

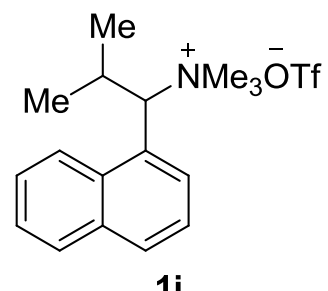

${ }^{1} \mathrm{H}$ NMR (400 MHz, $\mathrm{CDCl}_{3}$ )

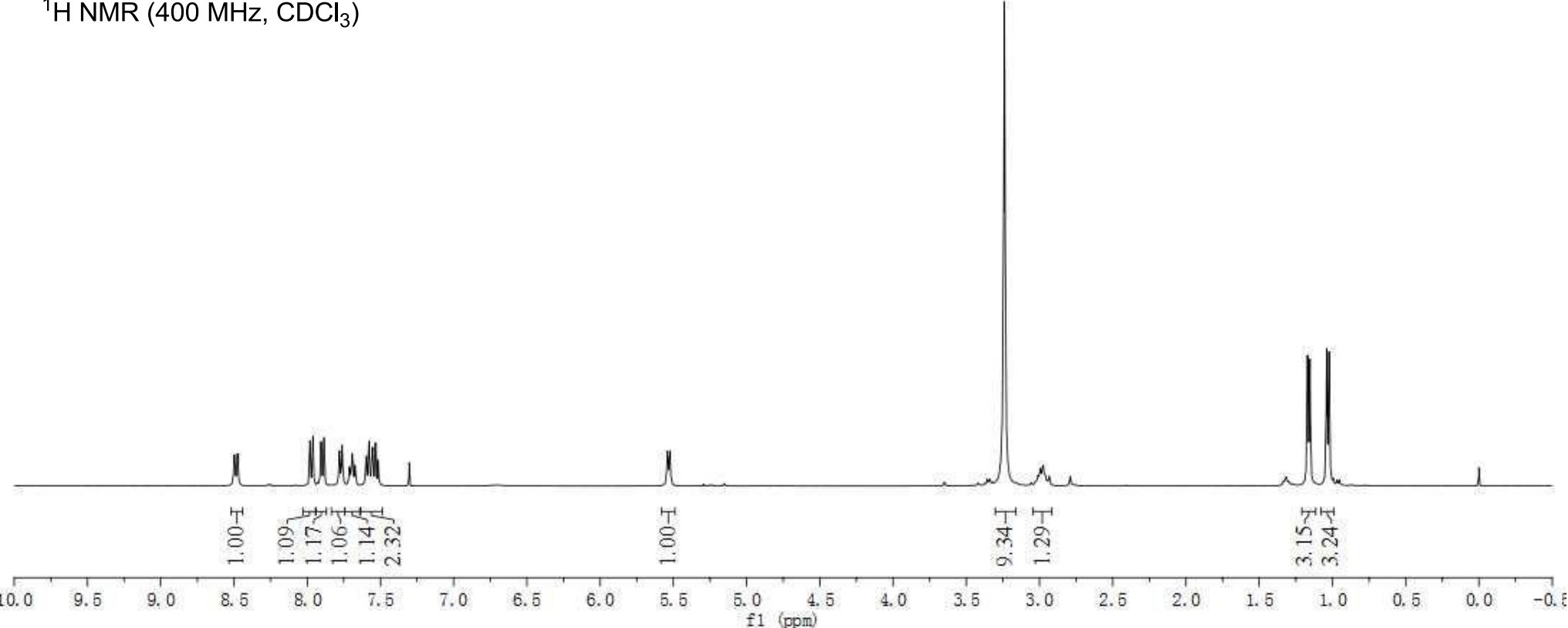



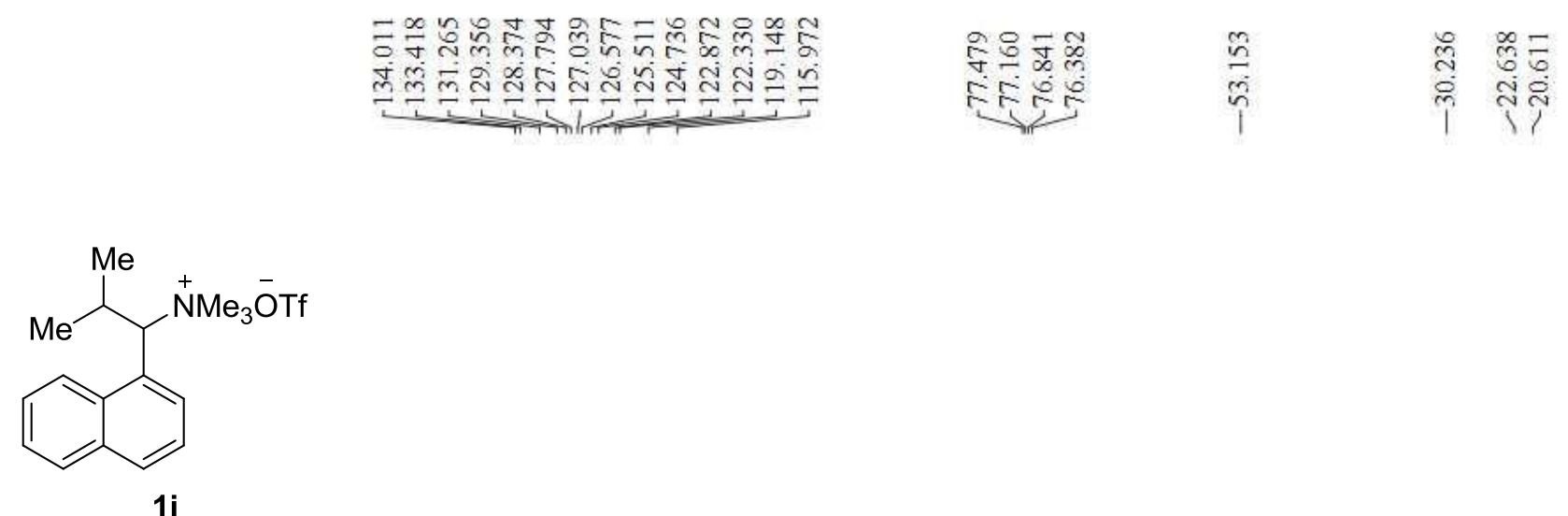

${ }^{13} \mathrm{C} \mathrm{NMR}\left(100 \mathrm{MHz}, \mathrm{CDCl}_{3}\right)$

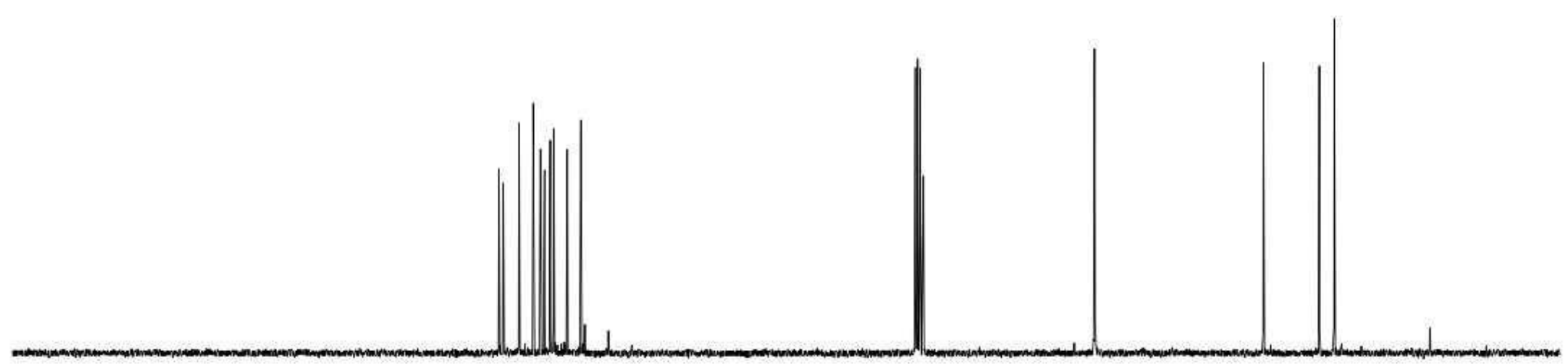

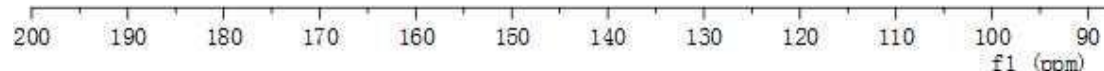




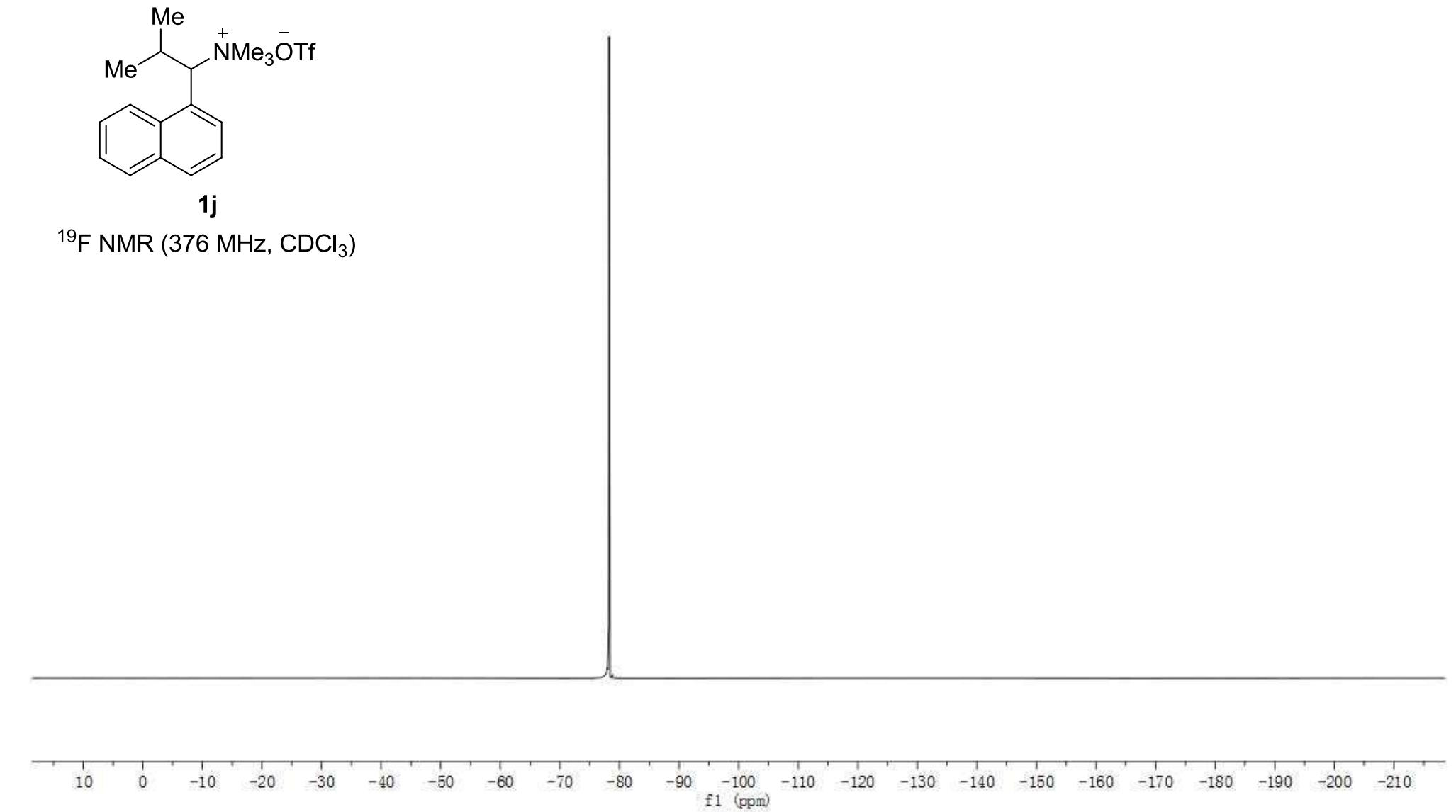

${ }^{19} \mathrm{~F} \mathrm{NMR}\left(376 \mathrm{MHz}, \mathrm{CDCl}_{3}\right.$ )

: 


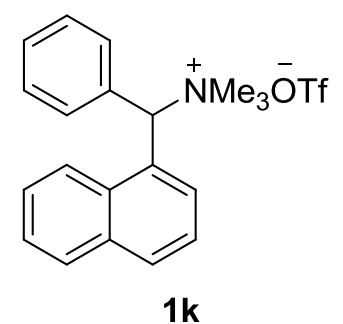

${ }^{1} \mathrm{H}$ NMR $\left(400 \mathrm{MHz}, \mathrm{CDCl}_{3}\right.$ )

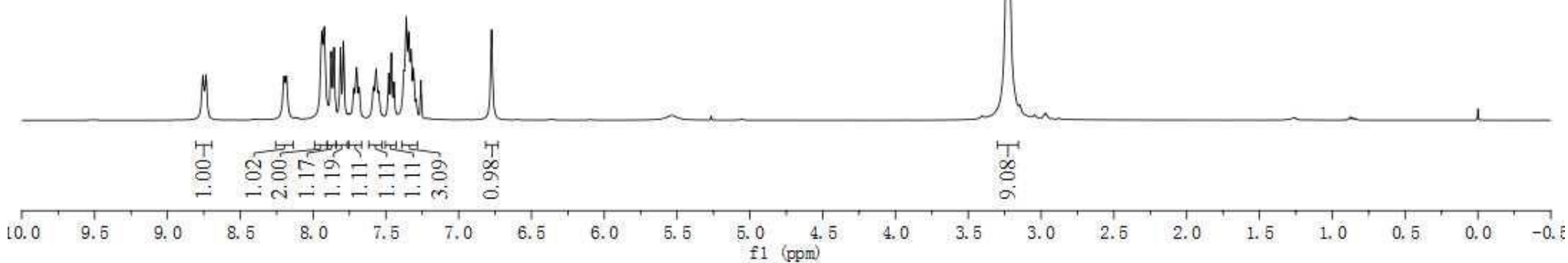




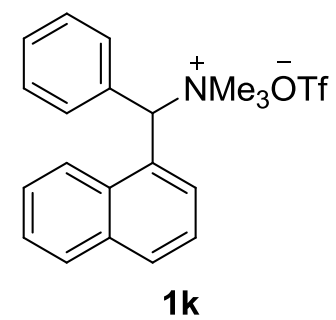

${ }^{13} \mathrm{C} \mathrm{NMR}\left(100 \mathrm{MHz}, \mathrm{CDCl}_{3}\right)$

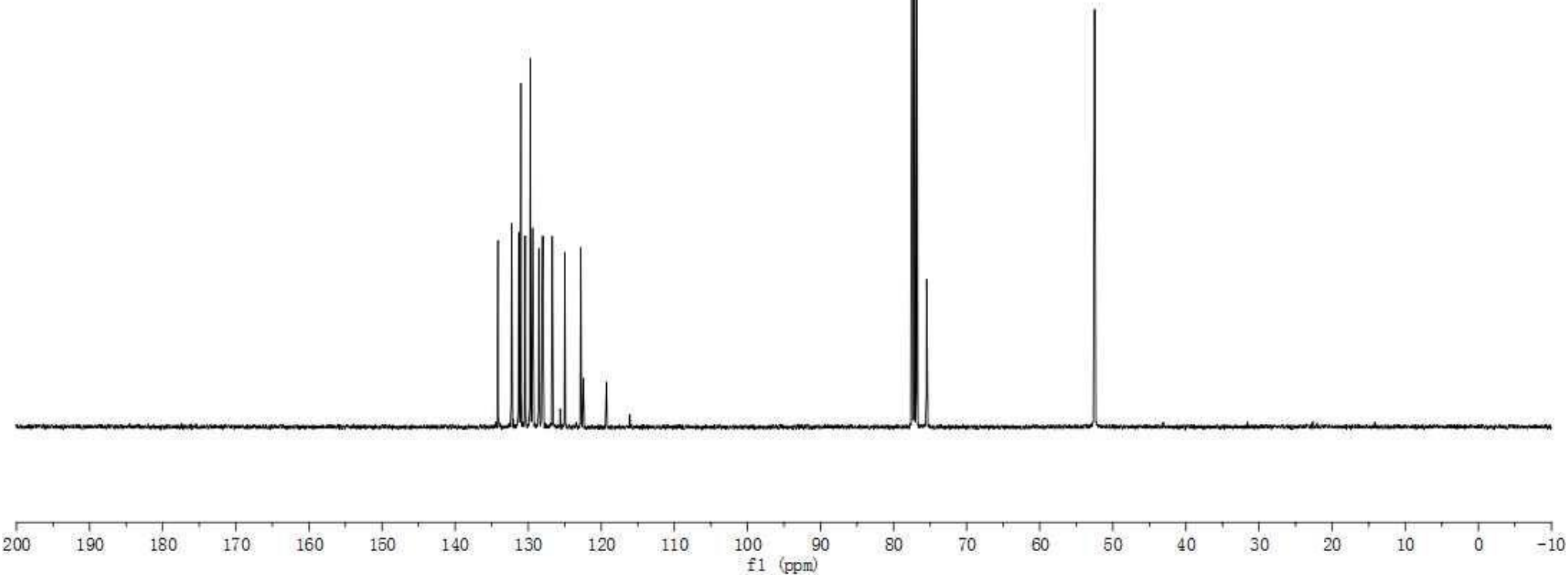




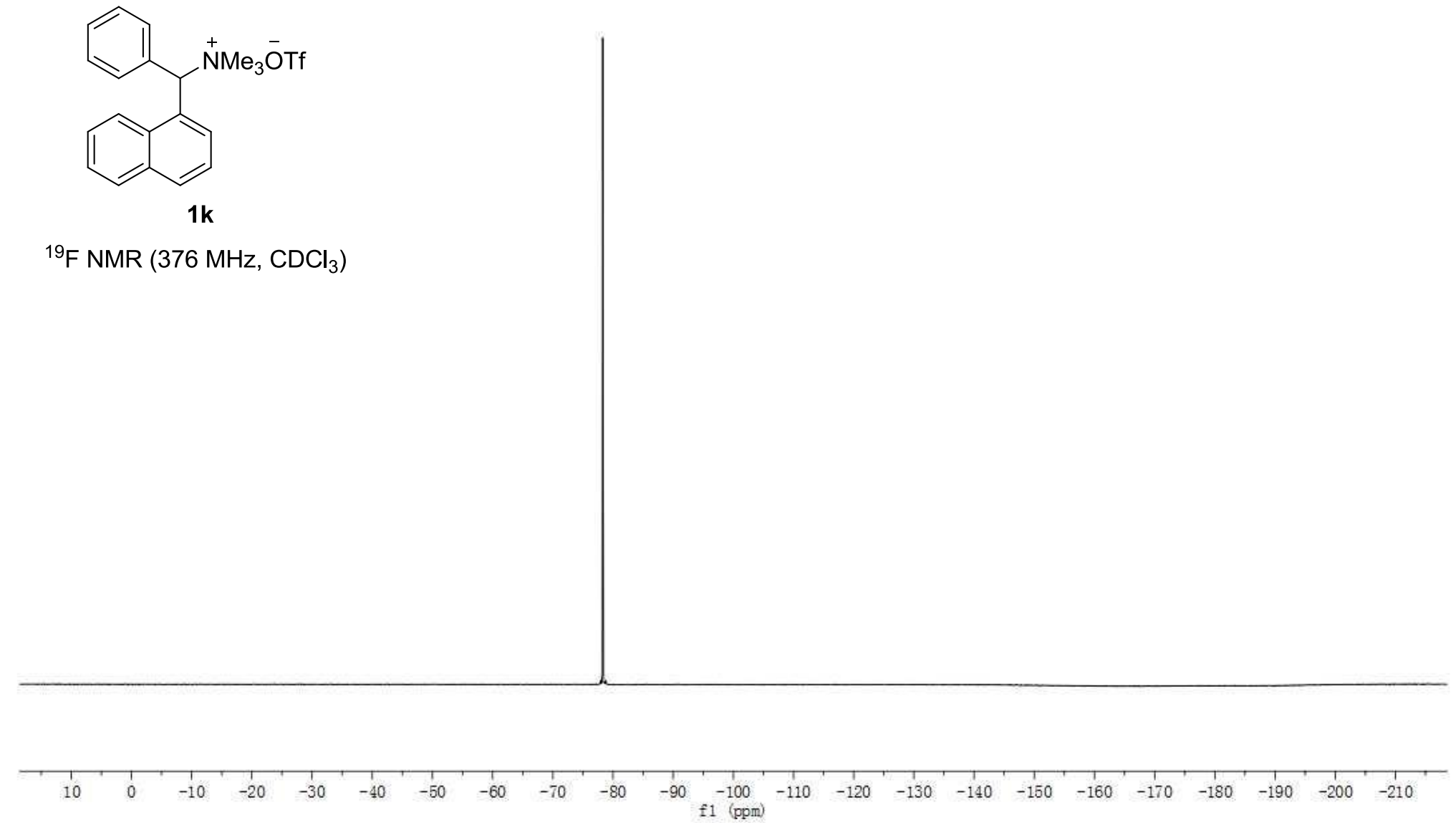




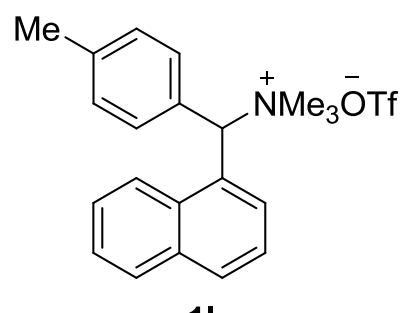

${ }^{1} \mathrm{H}$ NMR $\left(400 \mathrm{MHz}, \mathrm{CDCl}_{3}\right)$

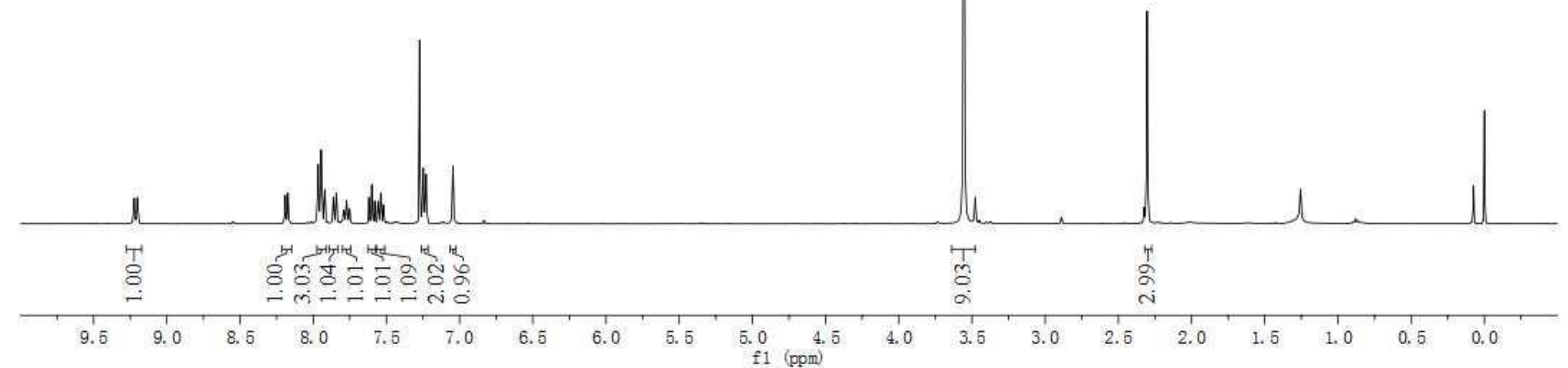




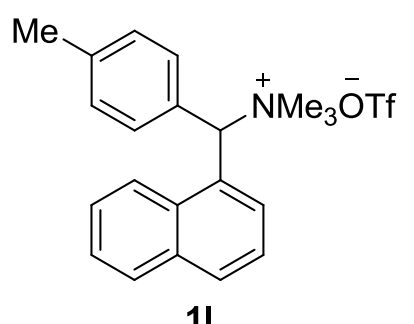

${ }^{13} \mathrm{C}$ NMR $\left(100 \mathrm{MHz}, \mathrm{CDCl}_{3}\right)$

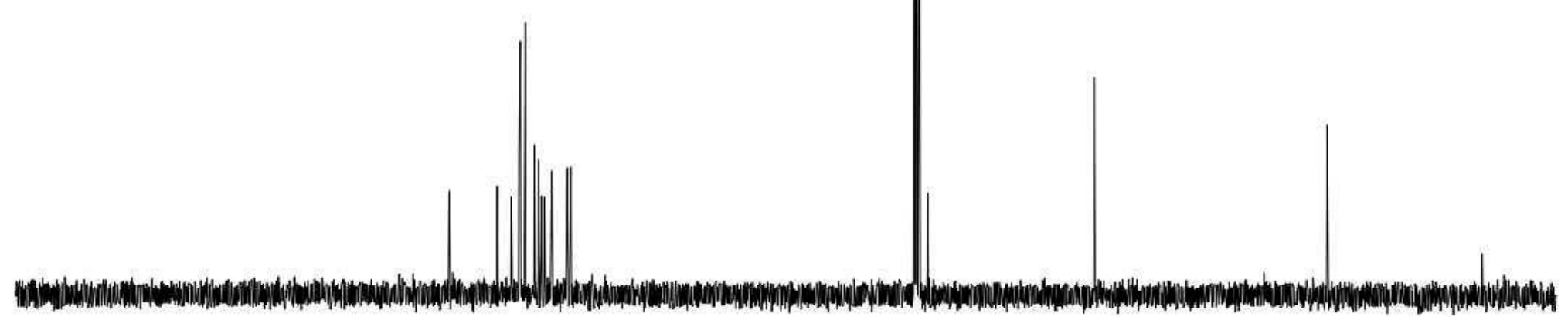

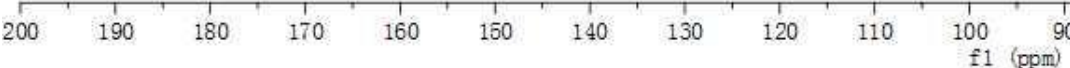




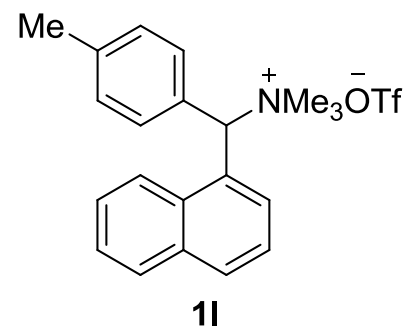

${ }^{19} \mathrm{~F} \mathrm{NMR}\left(376 \mathrm{MHz}, \mathrm{CDCl}_{3}\right)$

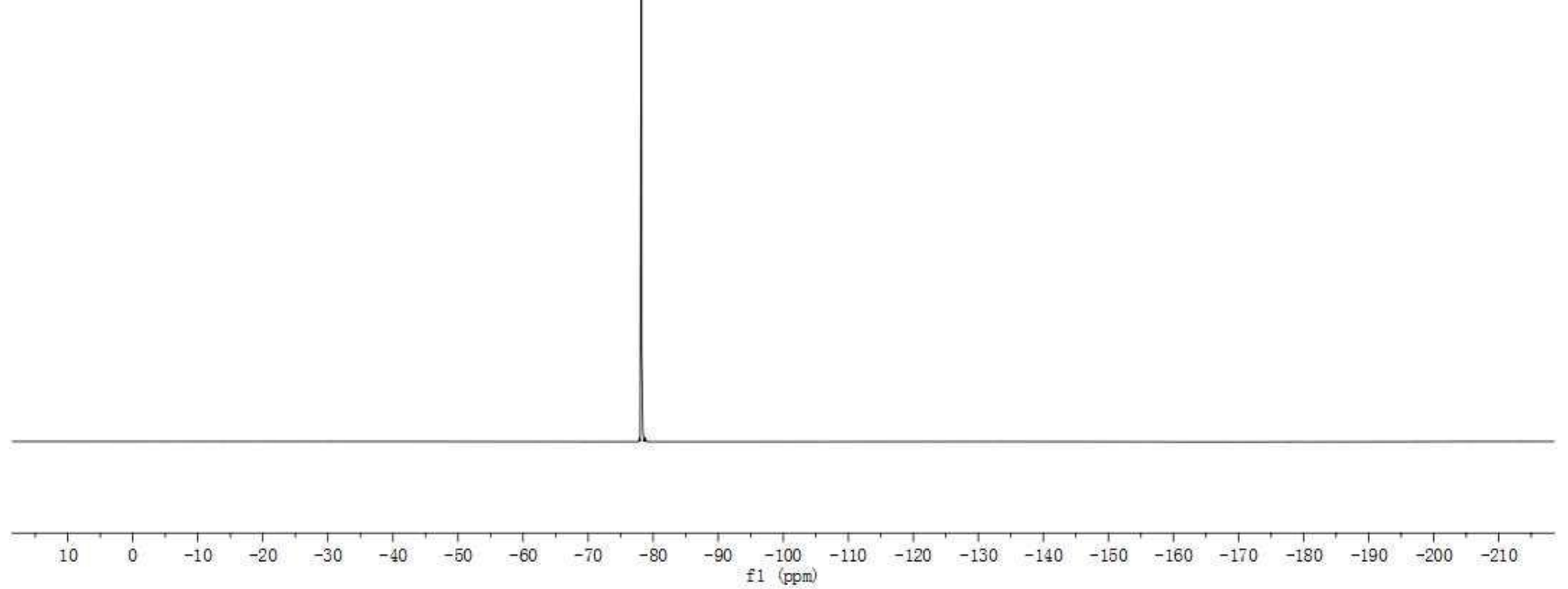




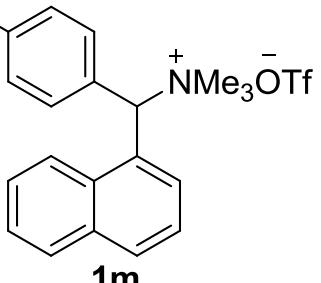

${ }^{1} \mathrm{H}$ NMR (400 MHz, CD $\left.{ }_{3} \mathrm{OD}\right)$

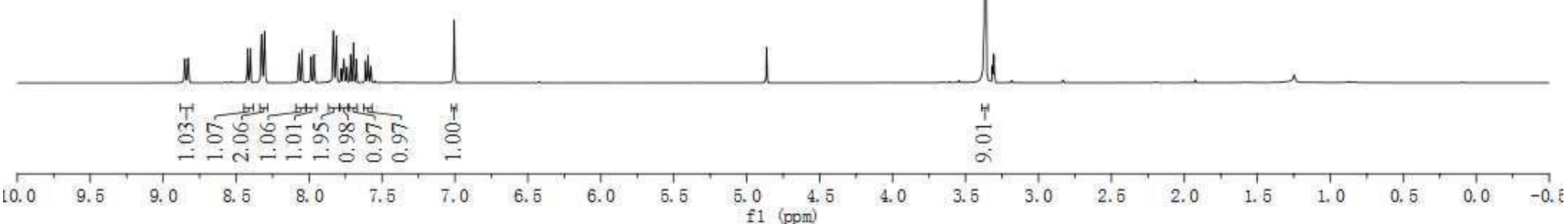




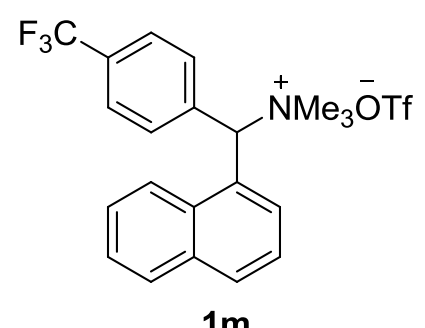

$1 \mathrm{~m}$

${ }^{13} \mathrm{C} \mathrm{NMR}\left(400 \mathrm{MHz}, \mathrm{CD}_{3} \mathrm{OD}\right)$

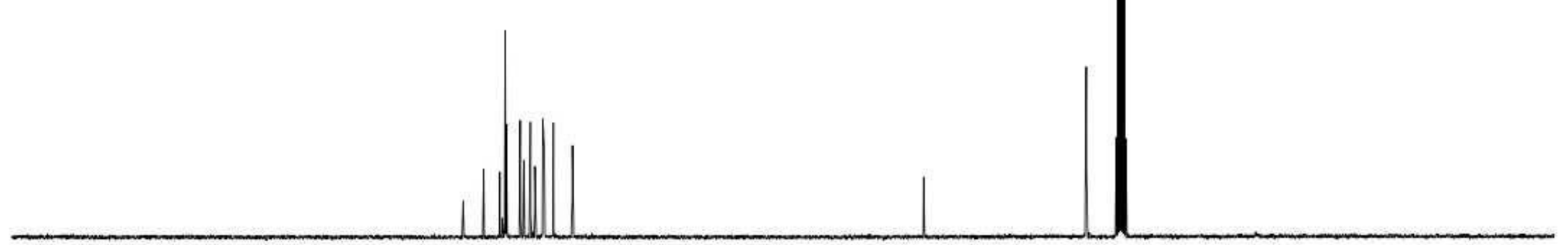

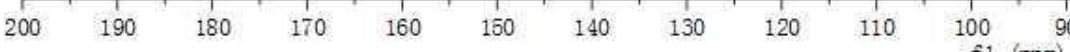




$$
\begin{array}{ll}
\overline{0} & 1 \\
0 & \frac{1}{0}
\end{array}
$$
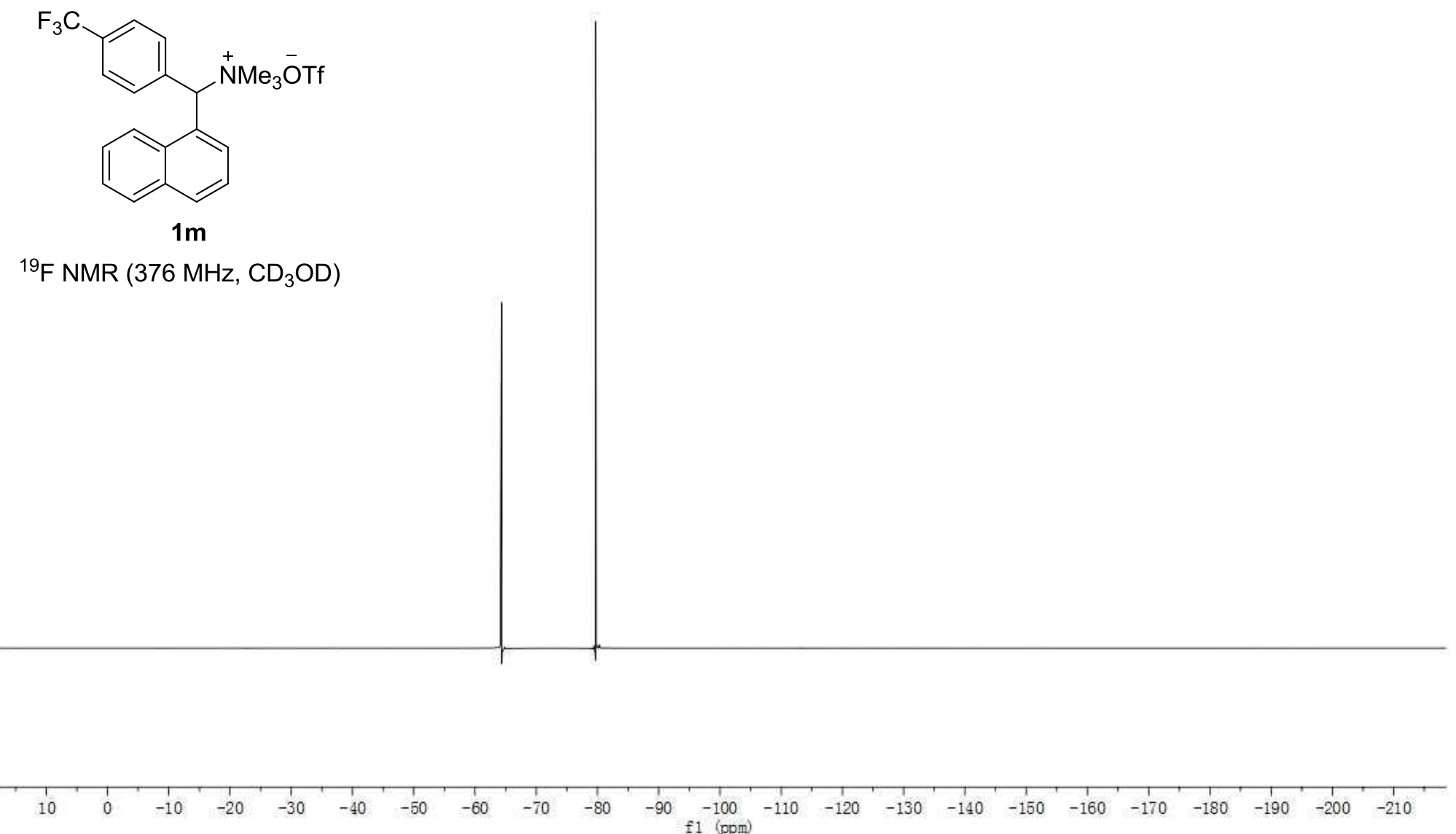


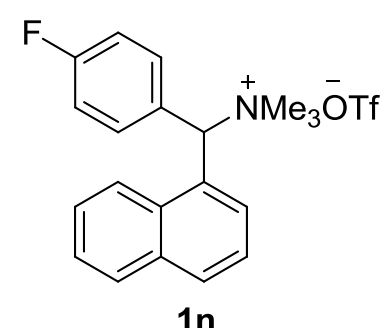

${ }^{1} \mathrm{H}$ NMR $\left(400 \mathrm{MHz}, \mathrm{CDCl}_{3}\right)$

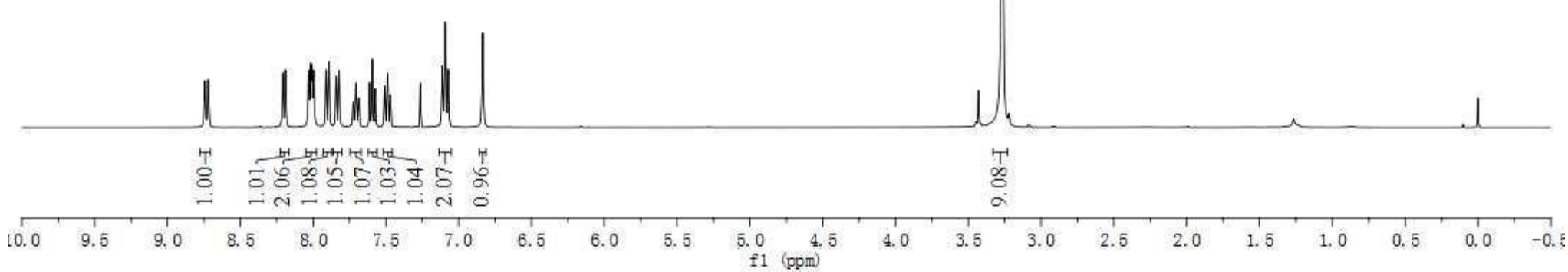




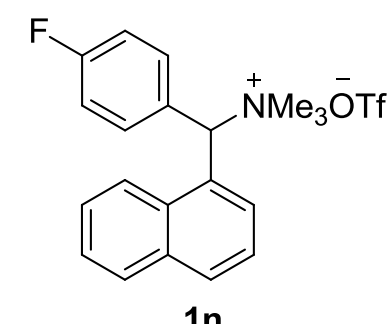

${ }^{13} \mathrm{C}$ NMR $\left(100 \mathrm{MHz}, \mathrm{CDCl}_{3}\right)$
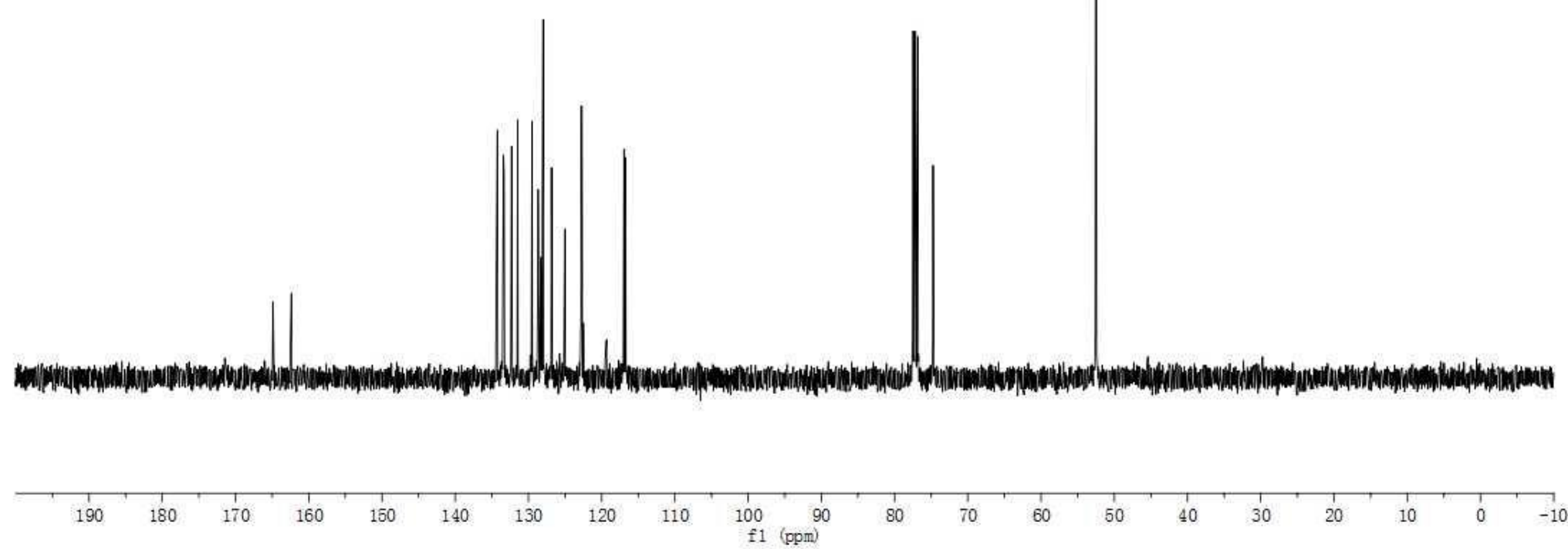


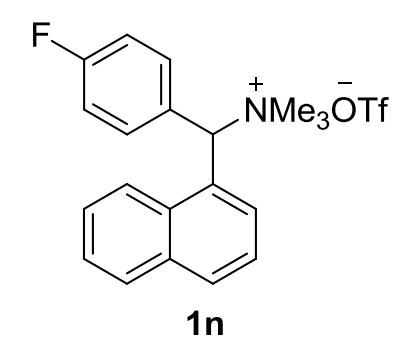

${ }^{19} \mathrm{~F}$ NMR (376 MHz, $\mathrm{CDCl}_{3}$ )

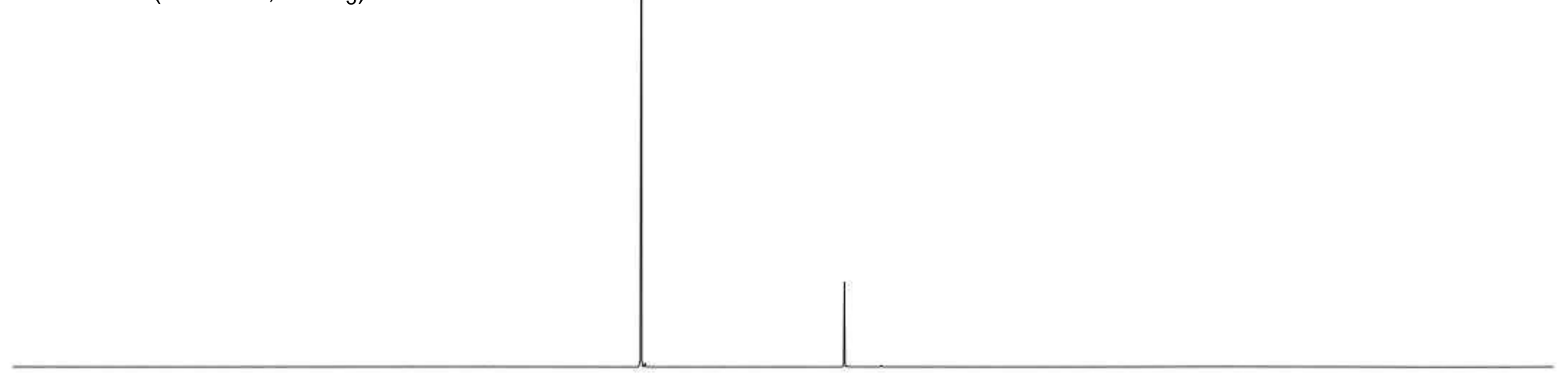




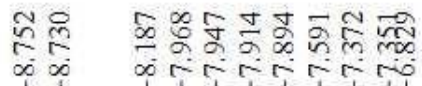 \\ $\infty \quad \infty \quad 55550$}

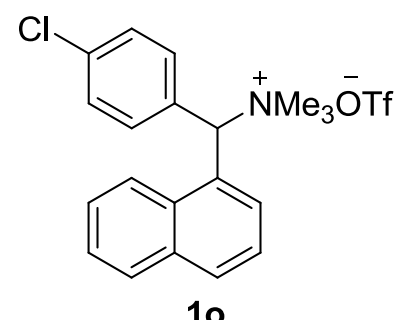

${ }^{1} \mathrm{H}$ NMR (400 MHz, $\left.\mathrm{CDCl}_{3}\right)$

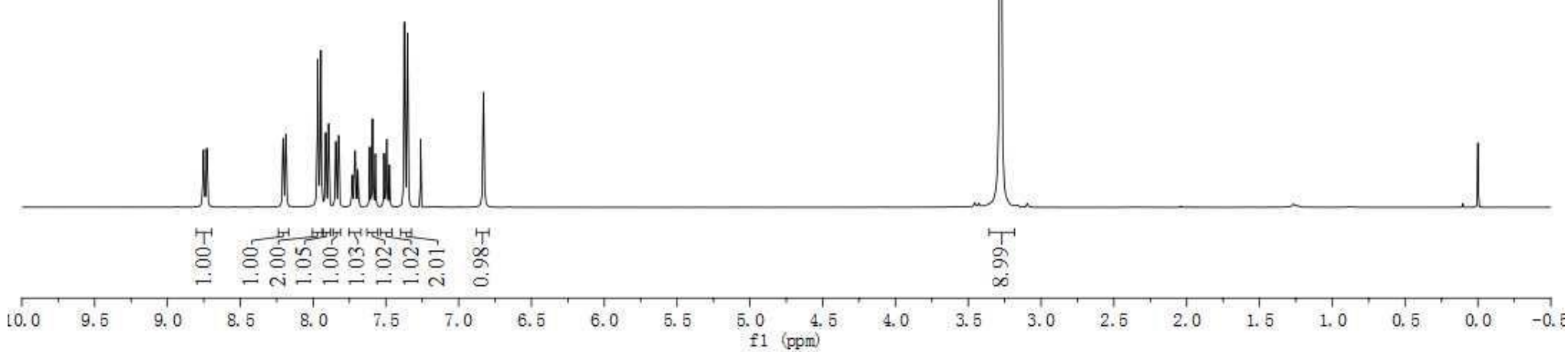




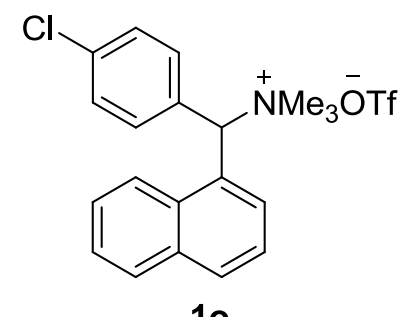

10

${ }^{13} \mathrm{C}$ NMR $\left(100 \mathrm{MHz}, \mathrm{CDCl}_{3}\right)$

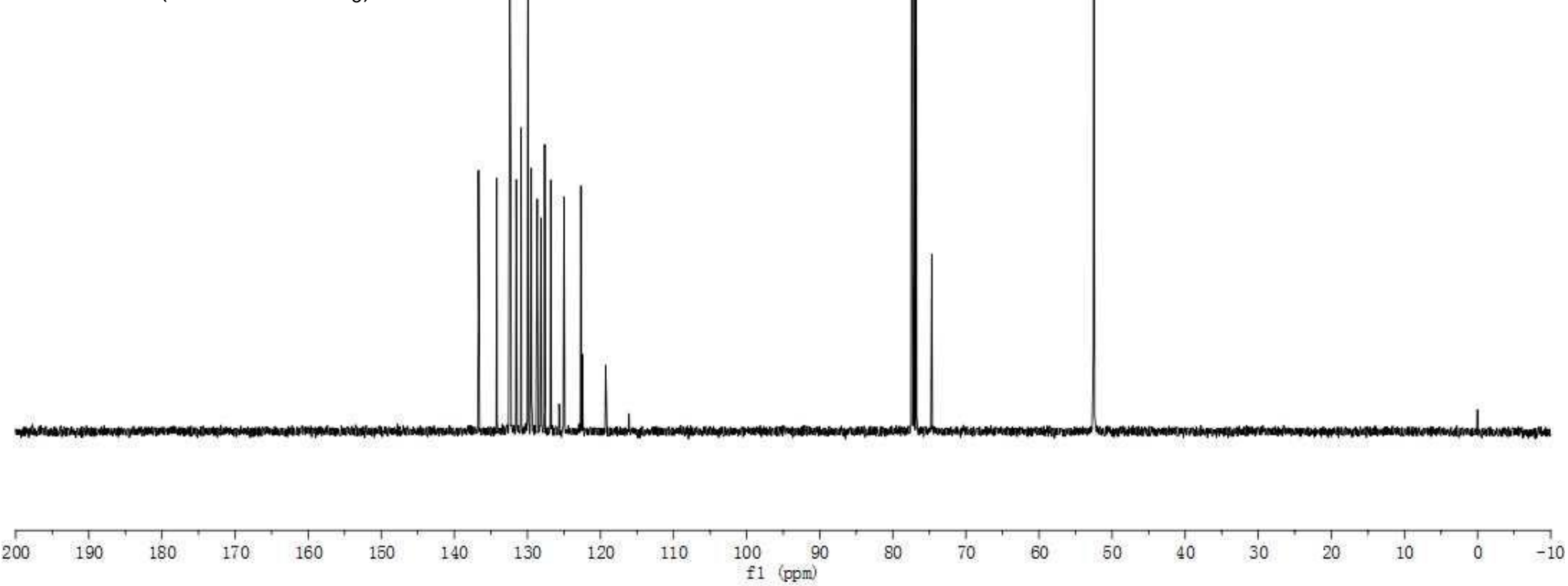




$$
\frac{\cdots}{m}
$$

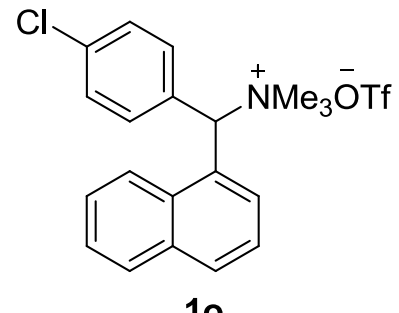

10

${ }^{19} \mathrm{~F} \mathrm{NMR}\left(376 \mathrm{MHz}, \mathrm{CDCl}_{3}\right)$

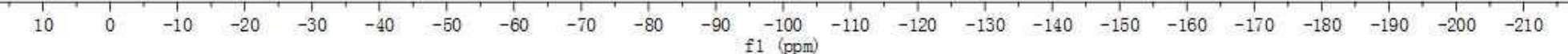



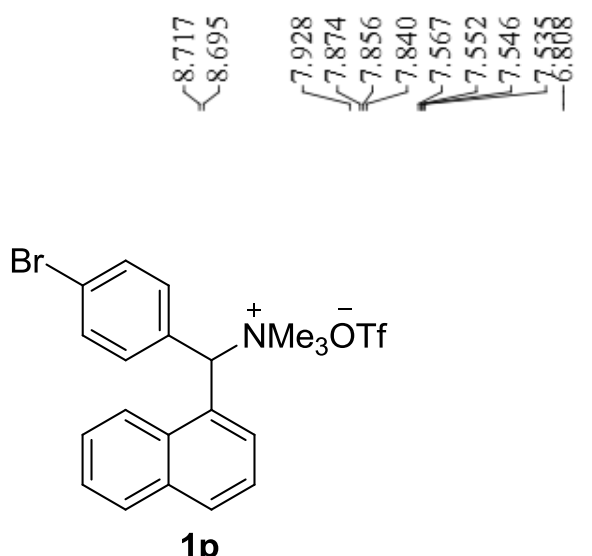

${ }^{1} \mathrm{H}$ NMR (400 MHz, $\mathrm{CDCl}_{3}$ )

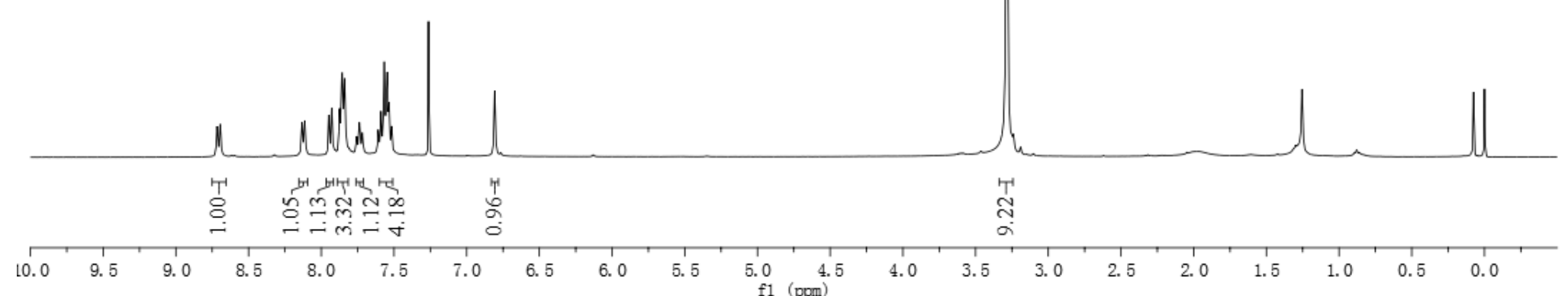




\section{1}

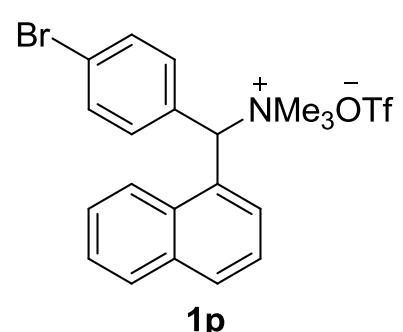

${ }^{13} \mathrm{C} \mathrm{NMR}\left(100 \mathrm{MHz}, \mathrm{CDCl}_{3}\right)$

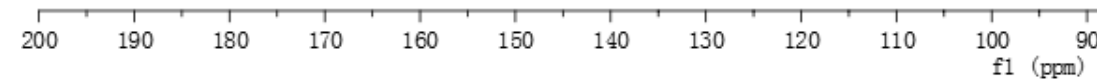




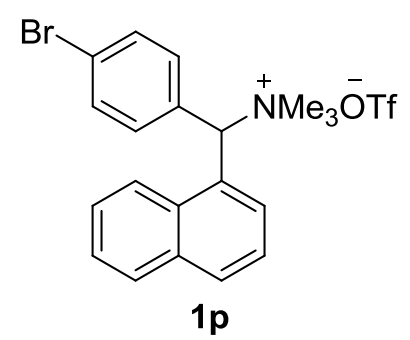

${ }^{19} \mathrm{~F} \mathrm{NMR}\left(376 \mathrm{MHz}, \mathrm{CDCl}_{3}\right)$

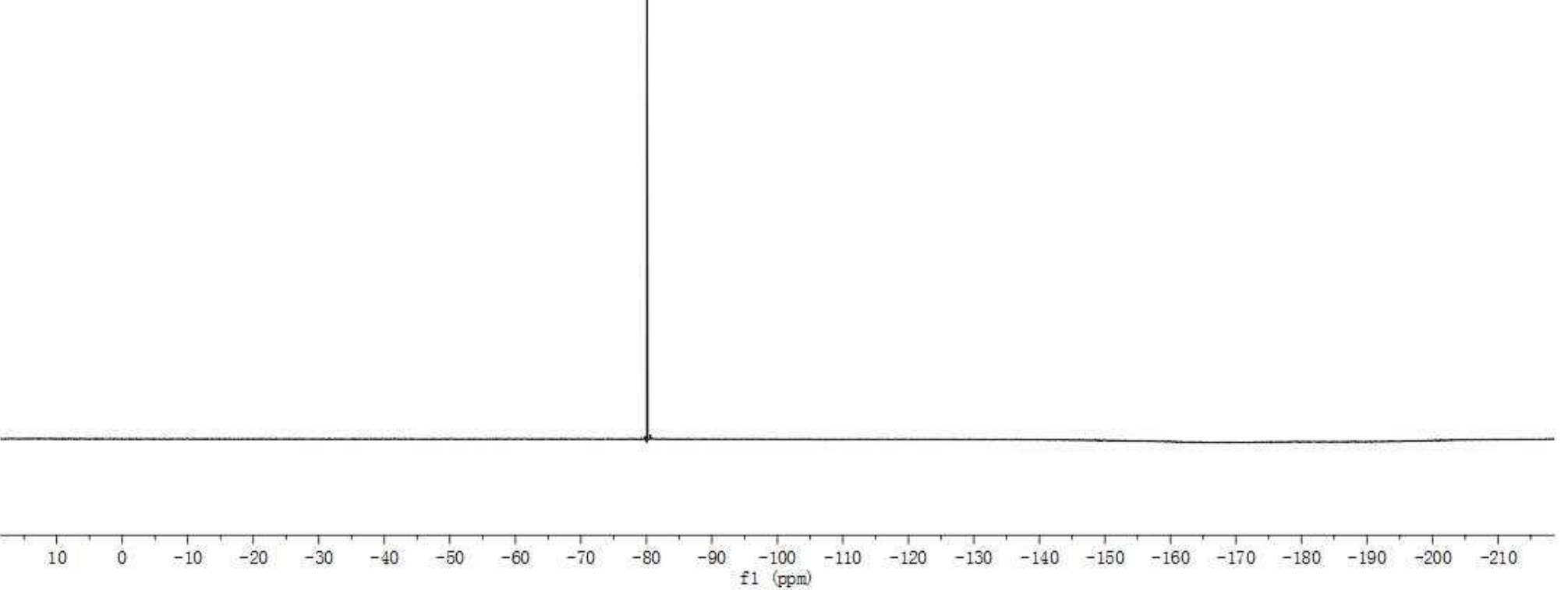




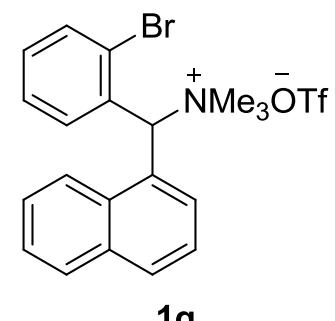

${ }^{1} \mathrm{H}$ NMR $\left(400 \mathrm{MHz}, \mathrm{CDCl}_{3}\right)$

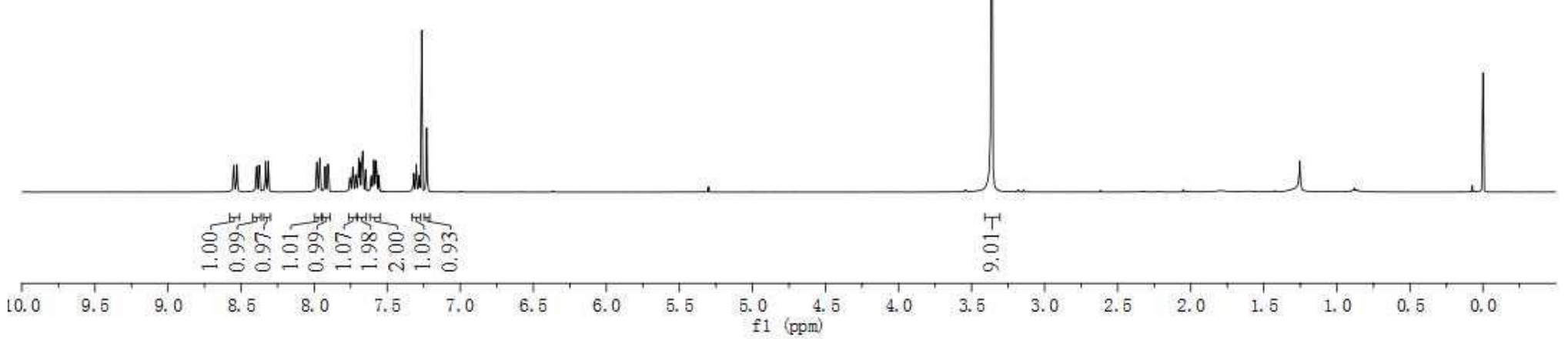




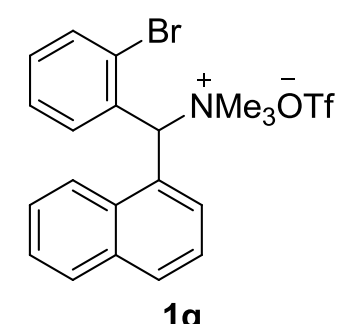

${ }^{13} \mathrm{C} \mathrm{NMR}\left(100 \mathrm{MHz}, \mathrm{CDCl}_{3}\right)$

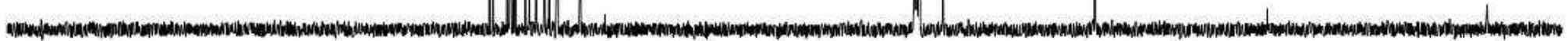

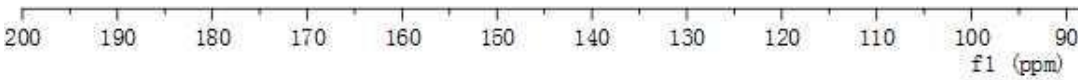




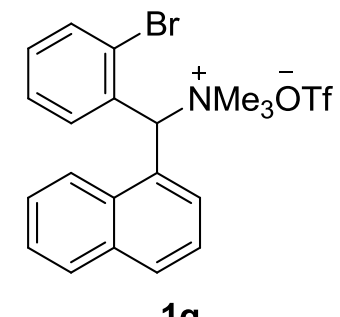

$\left.{ }^{19} \mathrm{~F} \mathrm{NMR} \mathrm{(376} \mathrm{MHz,} \mathrm{CDCl}_{3}\right)$

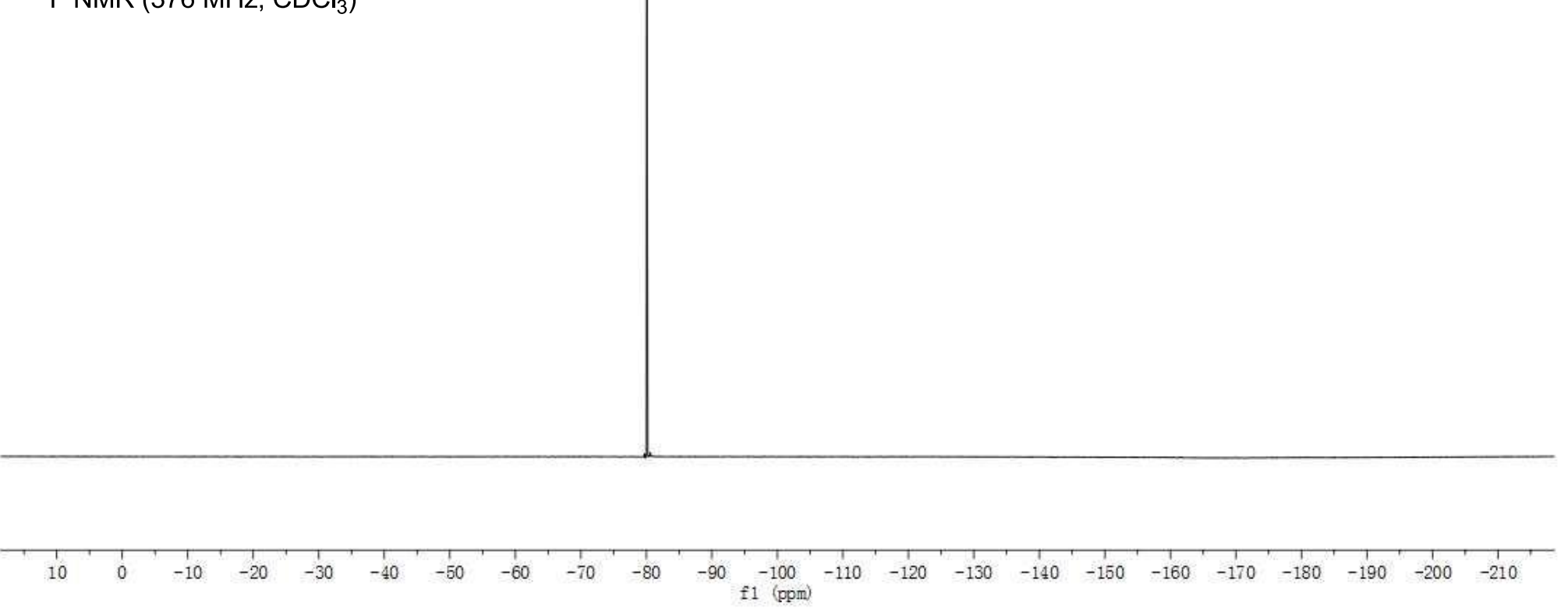




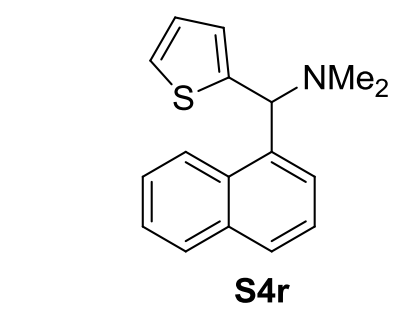

${ }^{1} \mathrm{H}$ NMR (400 MHz, $\left.\mathrm{CDCl}_{3}\right)$

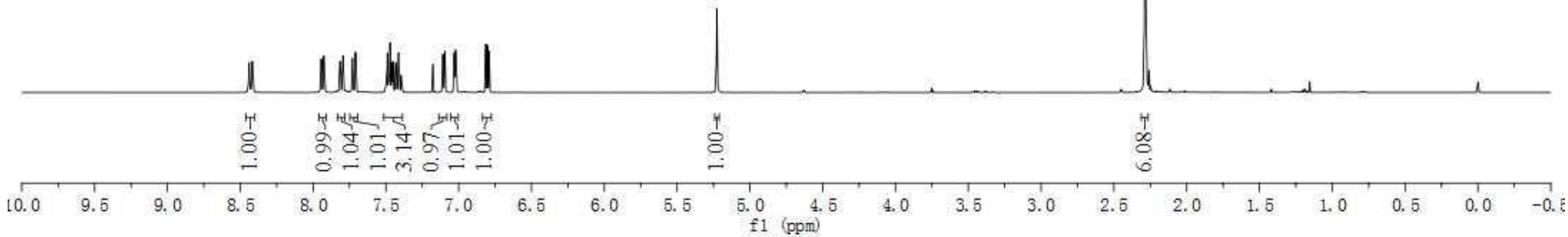




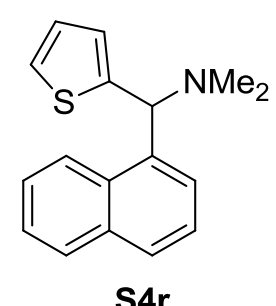

${ }^{13} \mathrm{C} \mathrm{NMR}\left(100 \mathrm{MHz}, \mathrm{CDCl}_{3}\right)$
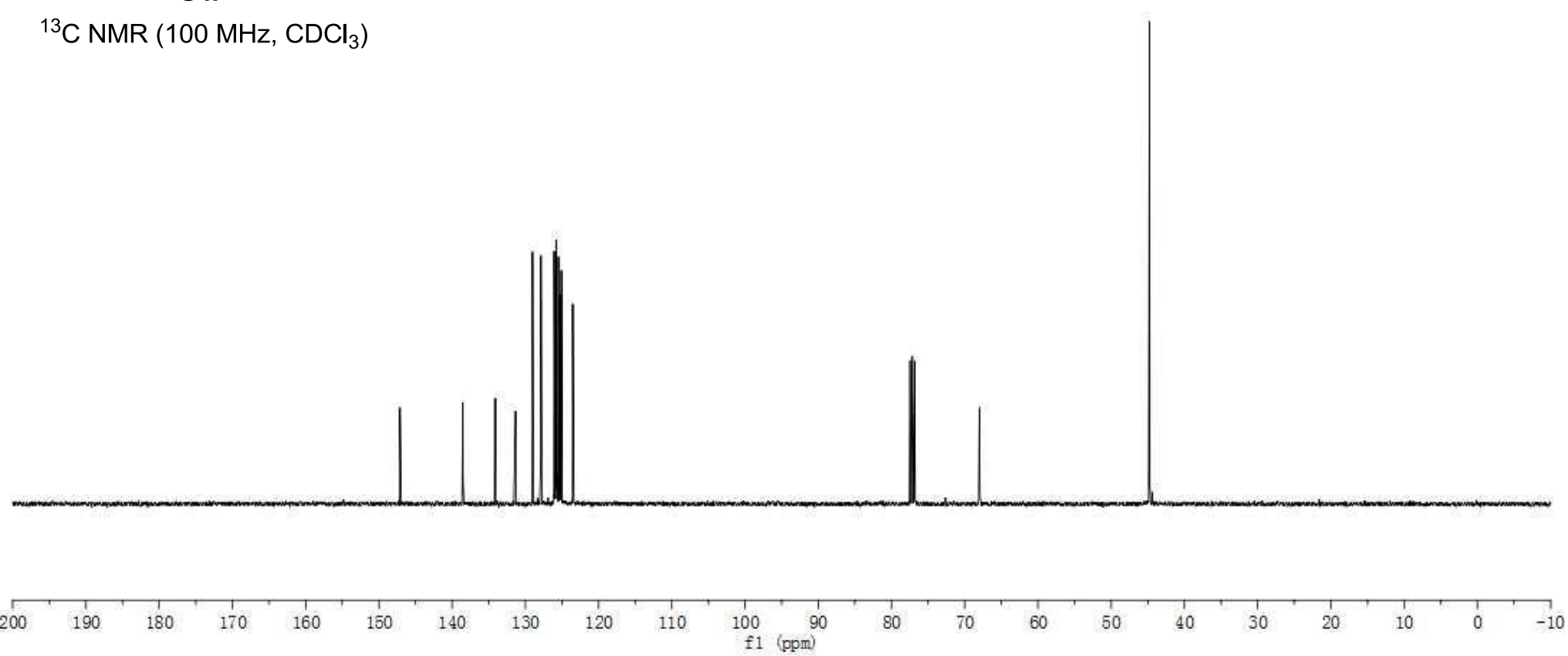


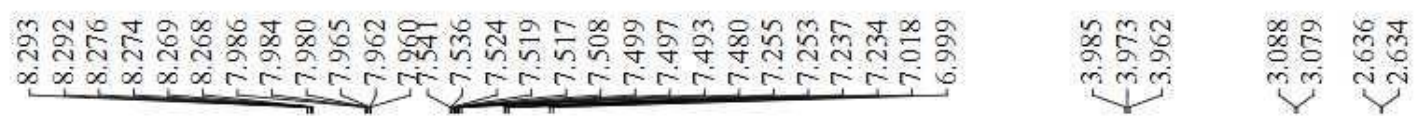

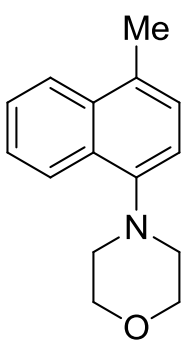

3a

${ }^{1} \mathrm{H} \mathrm{NMR}\left(400 \mathrm{MHz}, \mathrm{CDCl}_{3}\right.$ )

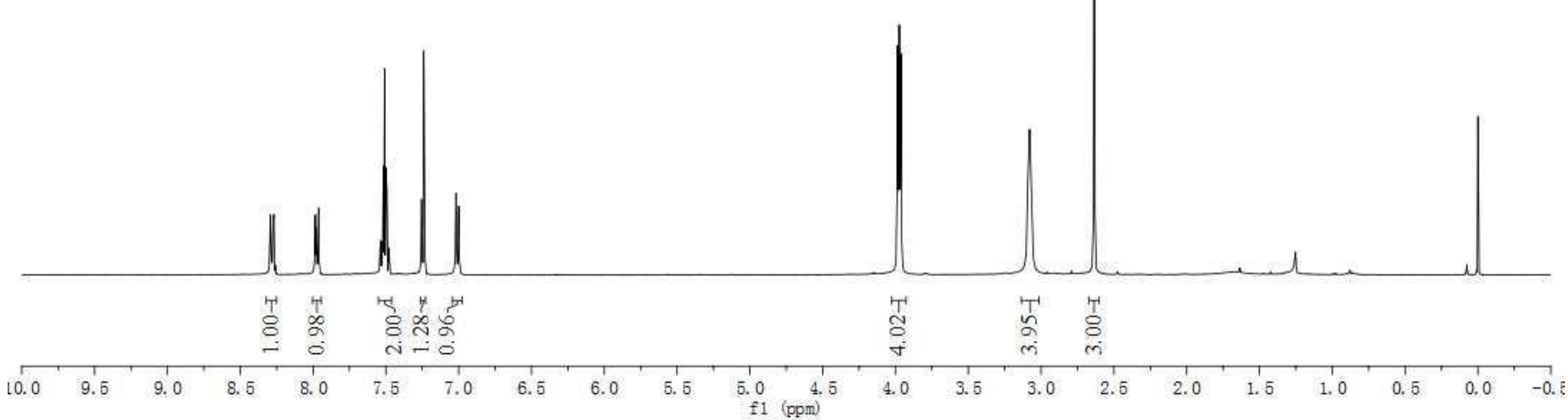




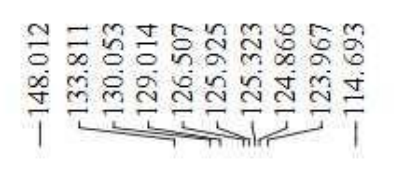

चुळ

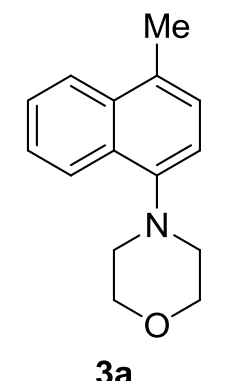

${ }^{13} \mathrm{C}$ NMR $\left(100 \mathrm{MHz}, \mathrm{CDCl}_{3}\right.$ )

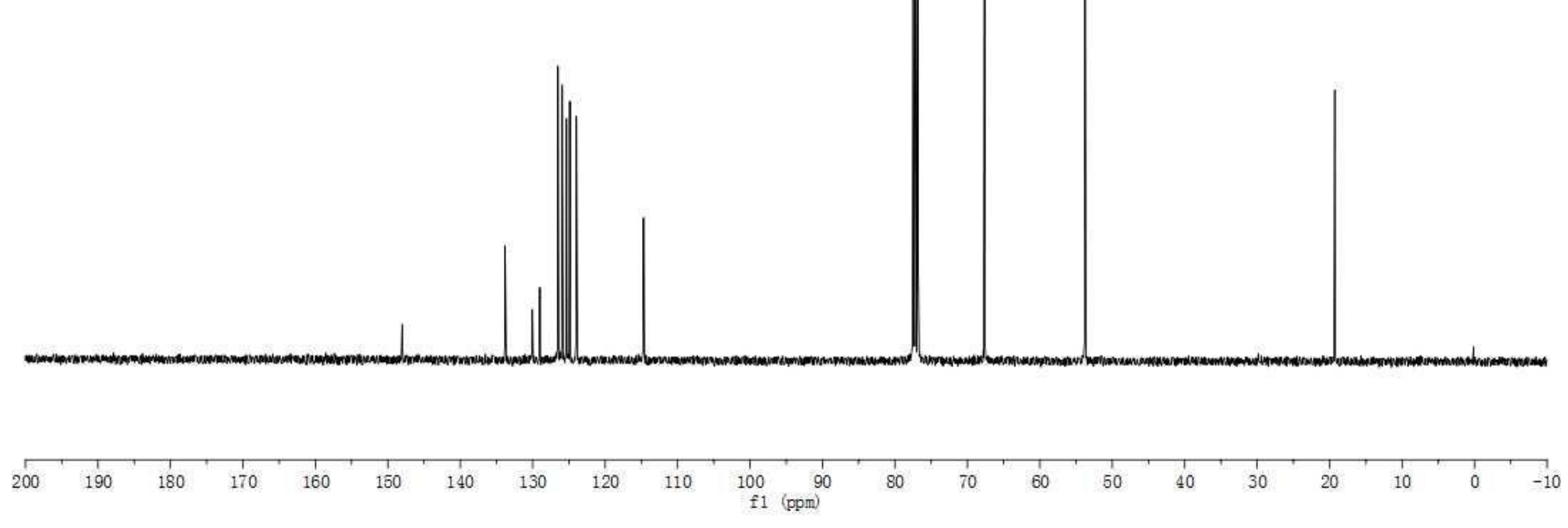




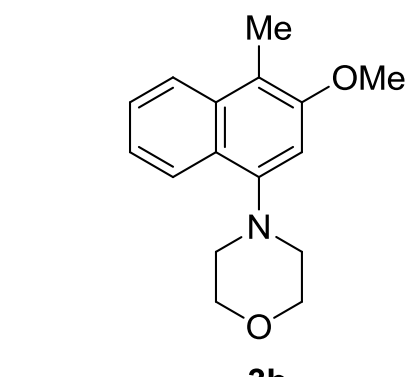

$3 b$

${ }^{1} \mathrm{H}$ NMR (400 MHz, $\mathrm{CDCl}_{3}$ )

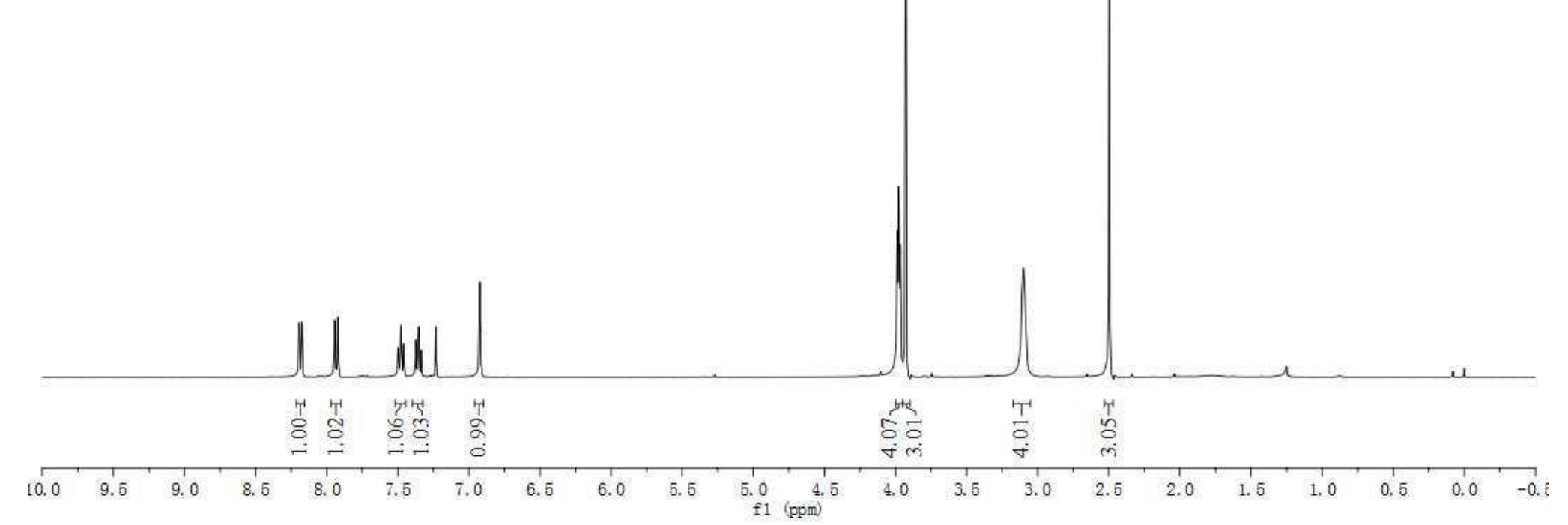



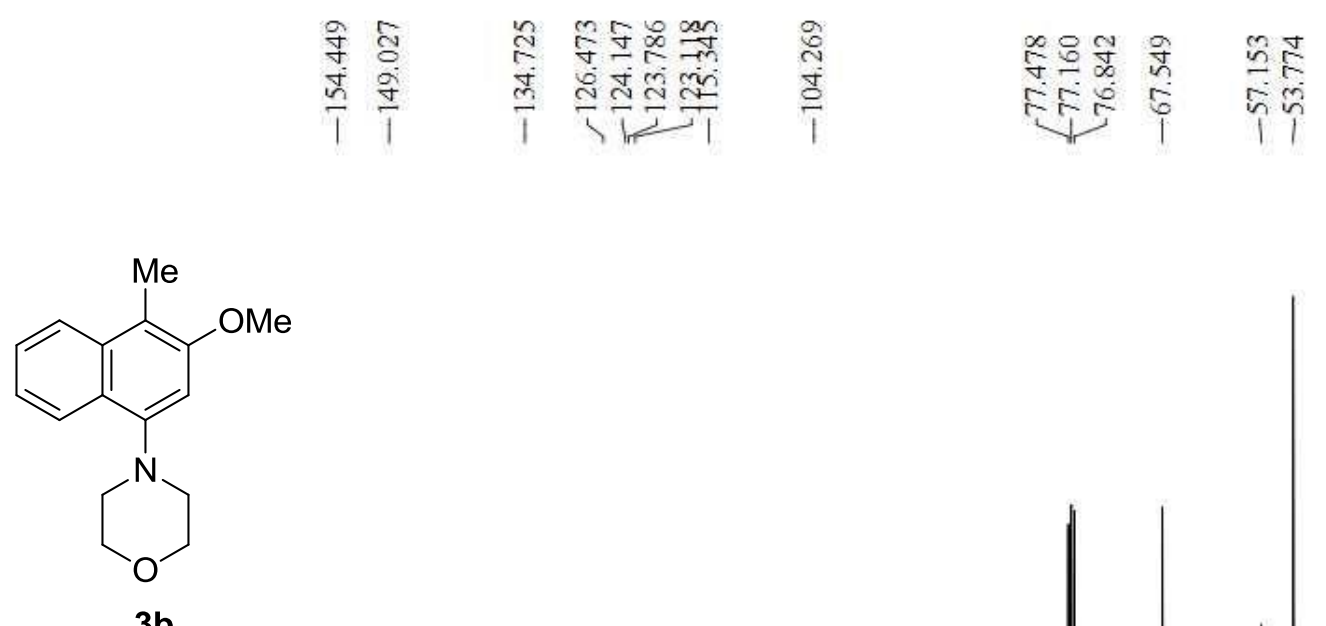

${ }^{13} \mathrm{C}$ NMR (100 MHz, $\mathrm{CDCl}_{3}$ )

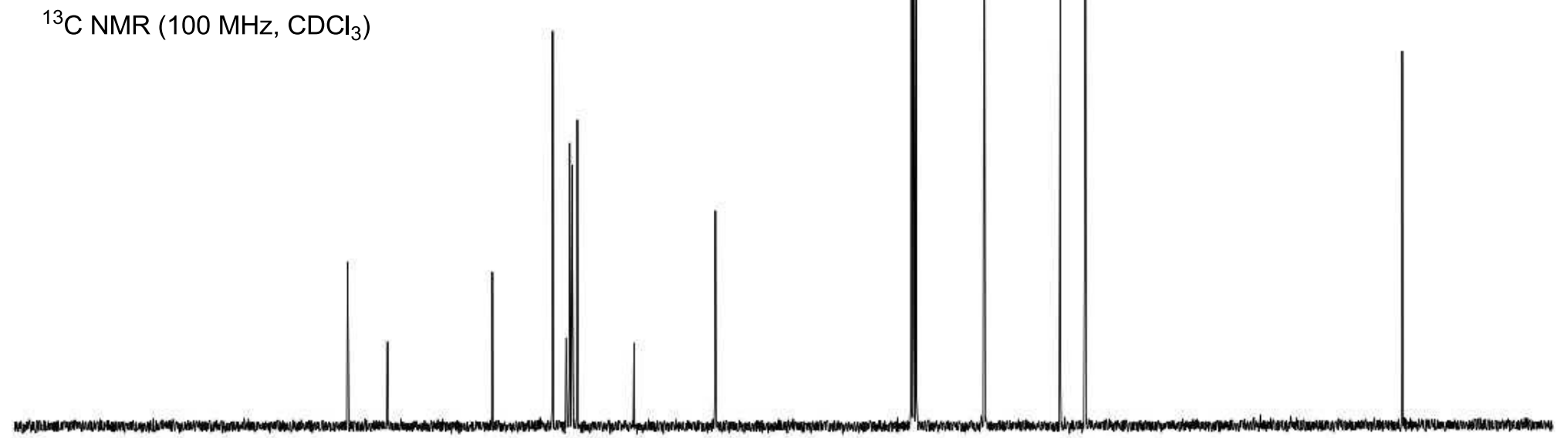

$200 \quad 190 \quad 180 \quad 170 \quad 160$

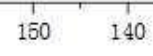

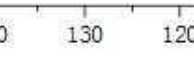

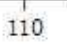

$100 \quad 90$

80

60

50

40 


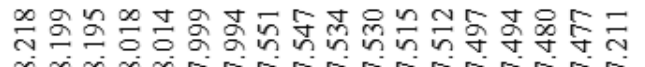

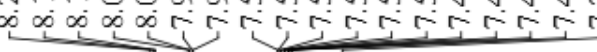

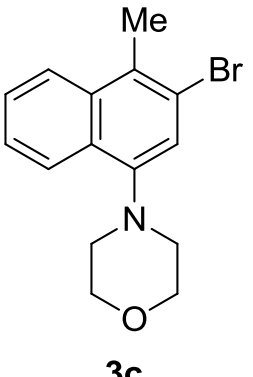

${ }^{1} \mathrm{H} \mathrm{NMR}\left(400 \mathrm{MHz}, \mathrm{CDCl}_{3}\right)$

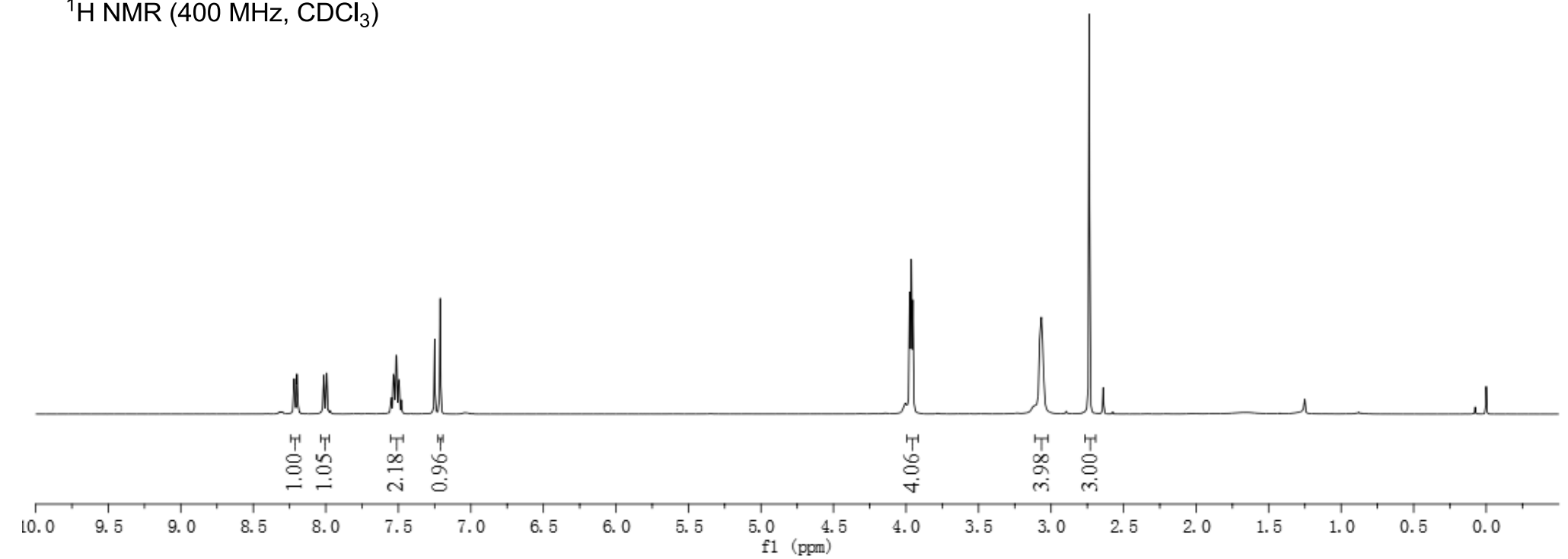



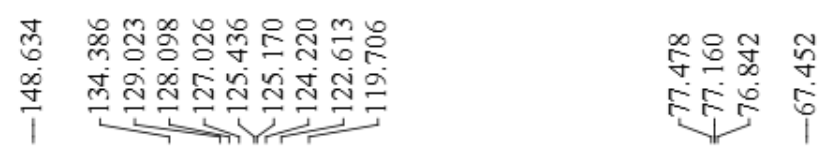

$n$
ñ
$n$

$n$
0
$\infty$
1

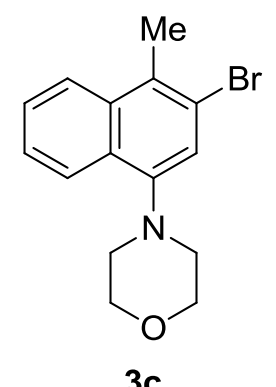

${ }^{13} \mathrm{C} \mathrm{NMR}\left(100 \mathrm{MHz}, \mathrm{CDCl}_{3}\right)$

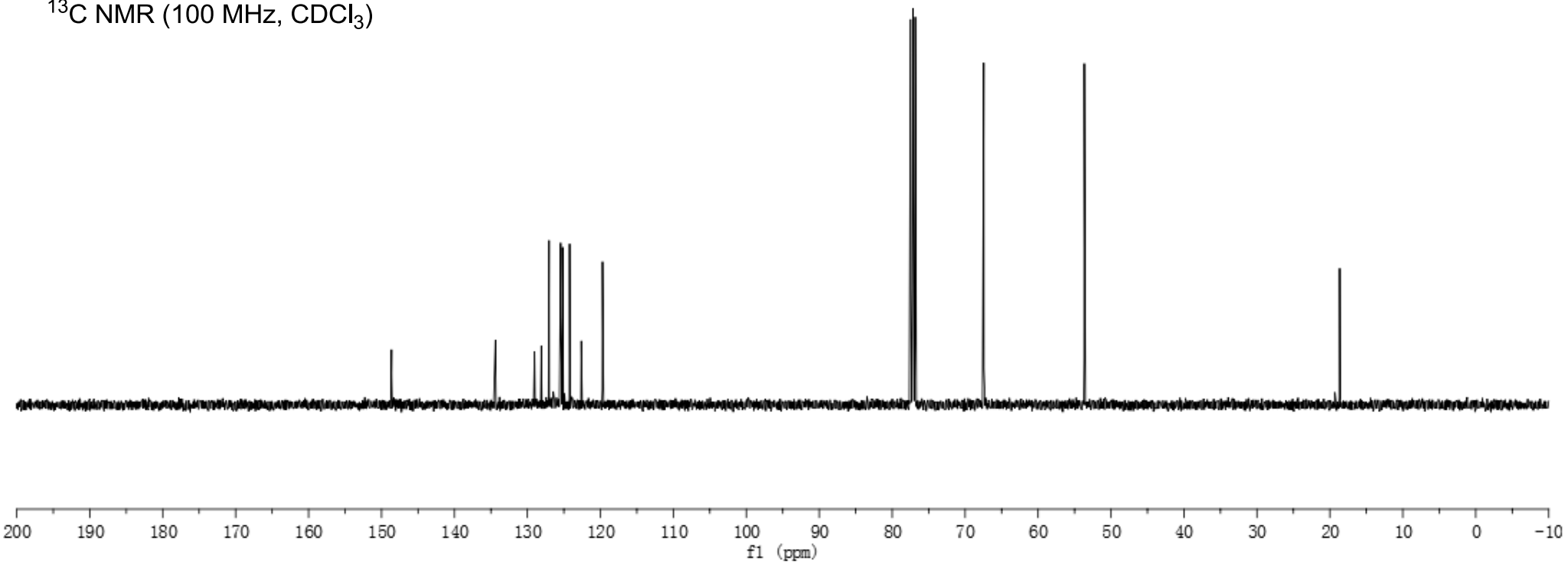




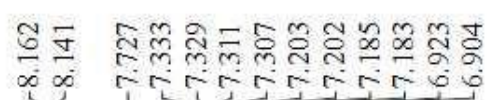

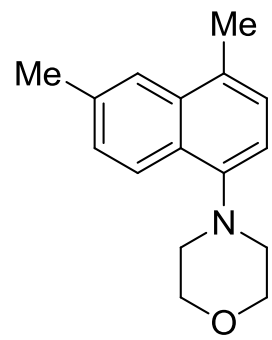

$3 d$

${ }^{1} \mathrm{H}$ NMR (400 MHz, $\mathrm{CDCl}_{3}$ )

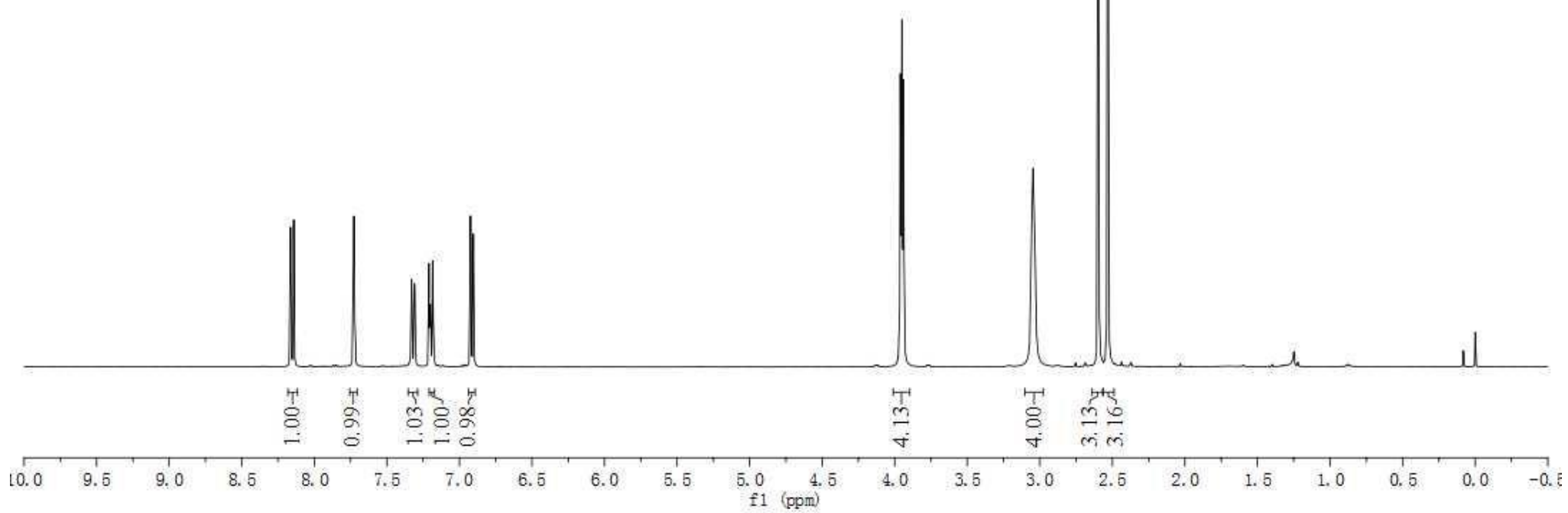



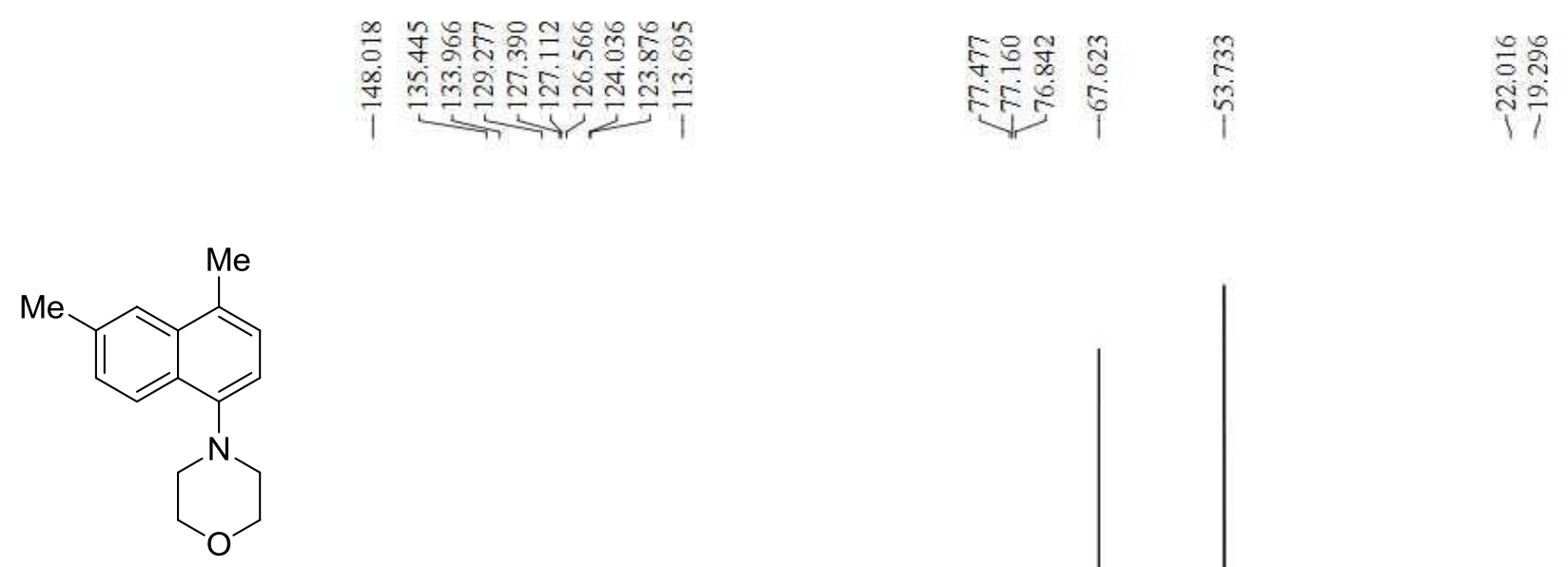

3d

${ }^{13} \mathrm{C} \mathrm{NMR}\left(100 \mathrm{MHz}, \mathrm{CDCl}_{3}\right)$

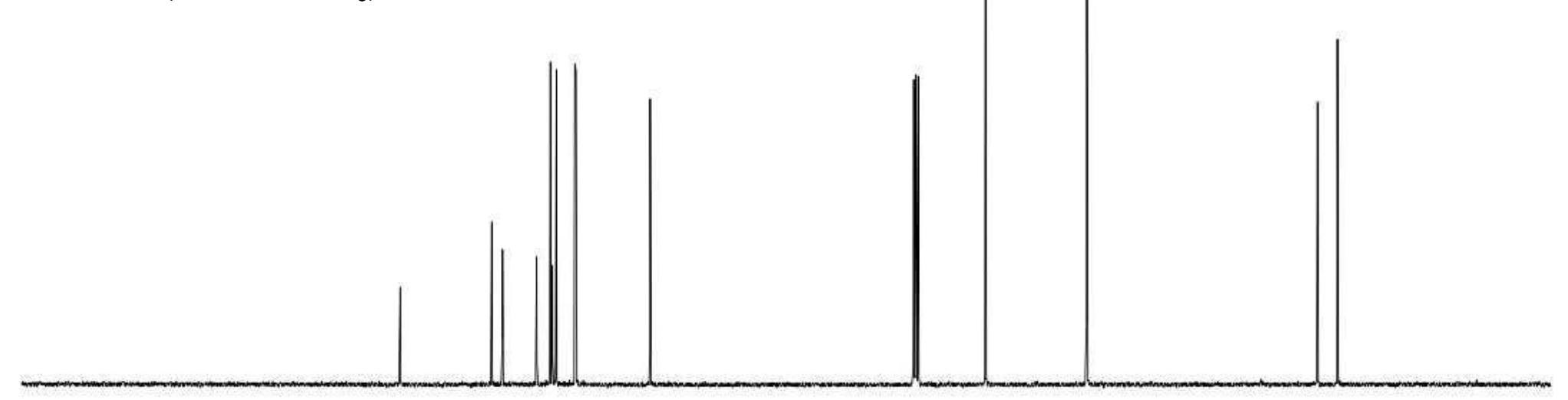

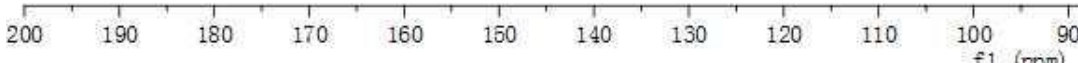




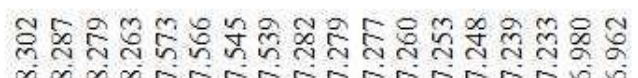

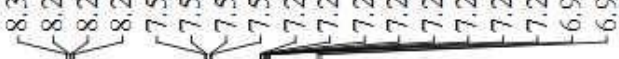

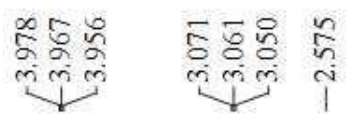

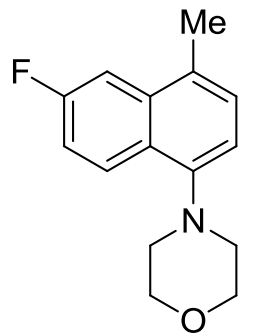

3 e

${ }^{1} \mathrm{H}$ NMR $\left(400 \mathrm{MHz}, \mathrm{CDCl}_{3}\right)$

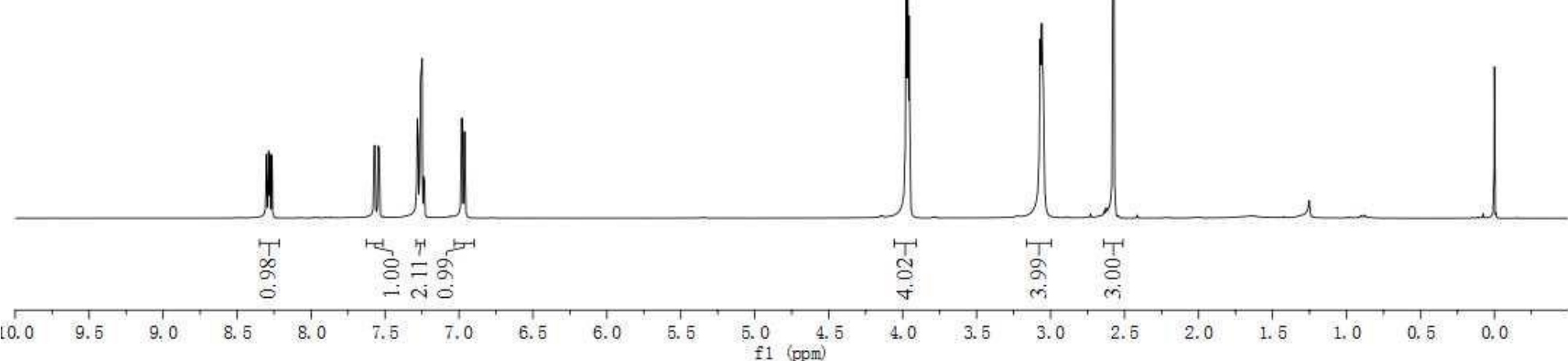




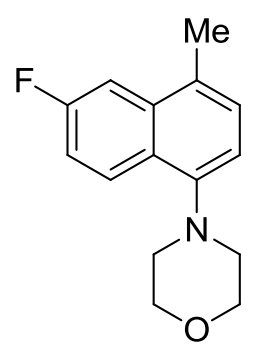

$3 e$

${ }^{13} \mathrm{C}$ NMR $\left(100 \mathrm{MHz}, \mathrm{CDCl}_{3}\right)$

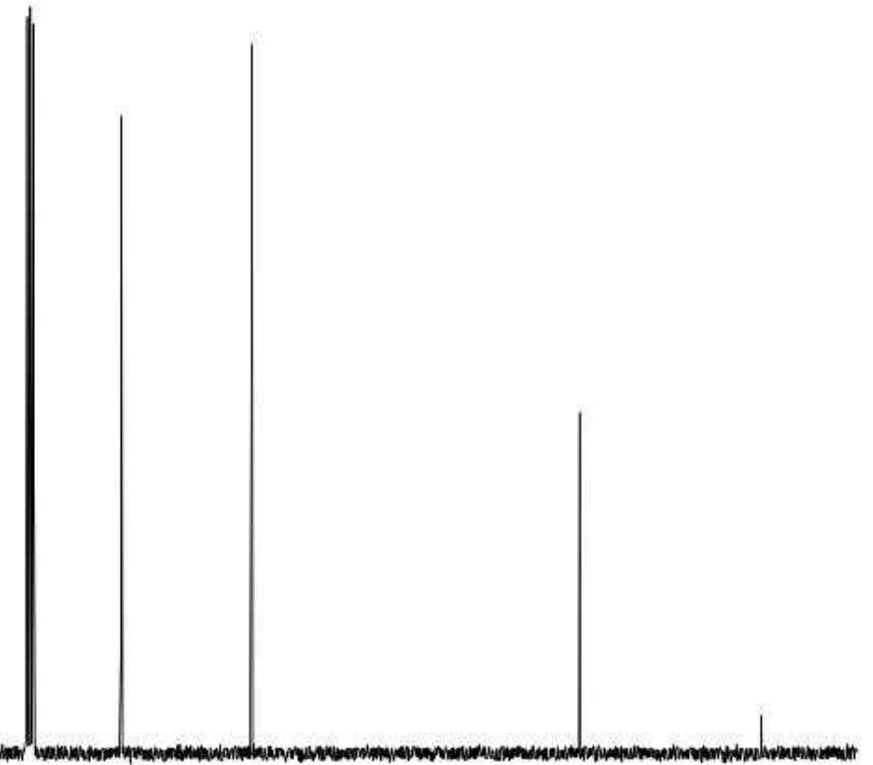

$200 \quad 190$

160

$150 \quad 140$

$130 \quad 120$

110

$100 \quad 90$ 


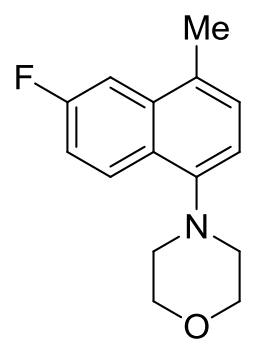

$3 e$

${ }^{19} \mathrm{~F} \mathrm{NMR}\left(376 \mathrm{MHz}, \mathrm{CDCl}_{3}\right)$

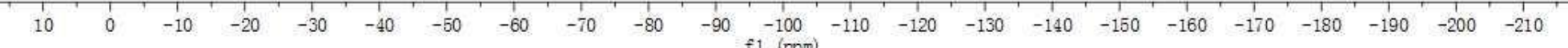



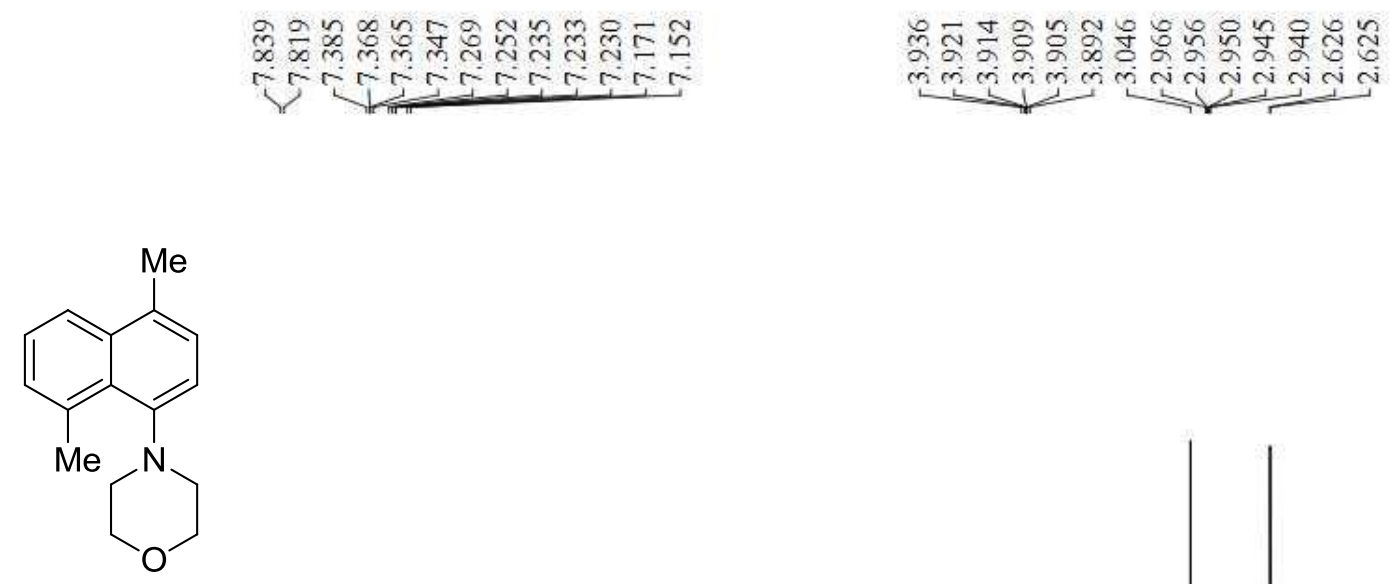

$3 f$

${ }^{1} \mathrm{H}$ NMR (400 MHz, $\mathrm{CDCl}_{3}$ )

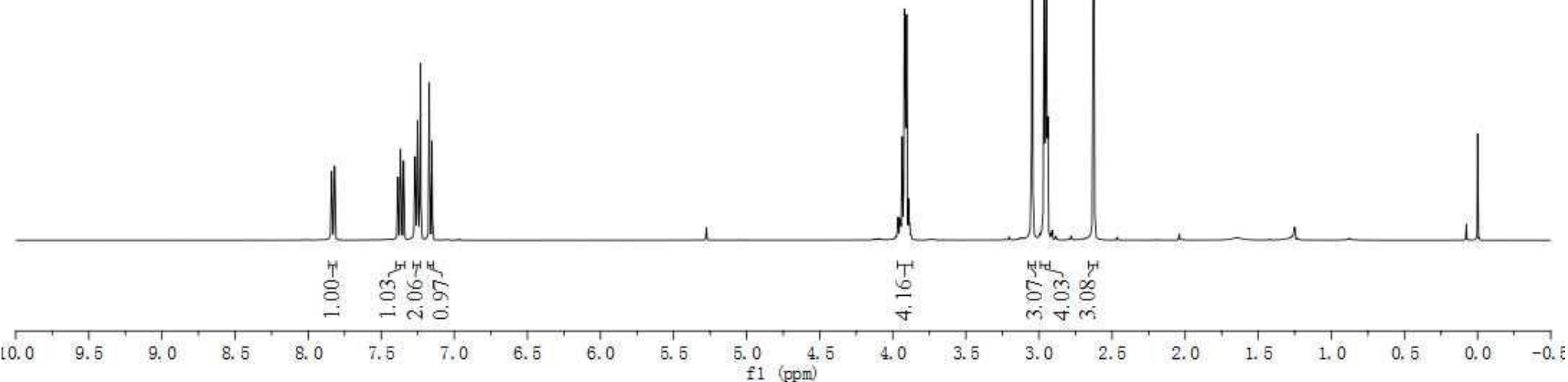




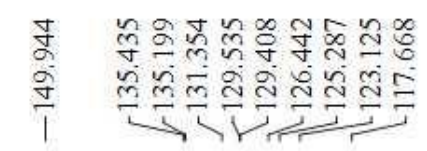

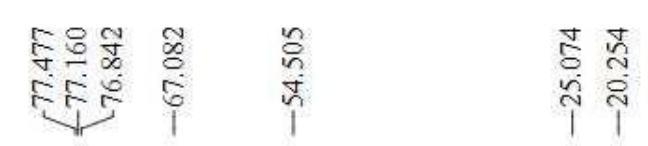

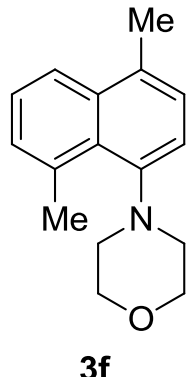

${ }^{13} \mathrm{C} \mathrm{NMR}\left(100 \mathrm{MHz}, \mathrm{CDCl}_{3}\right)$

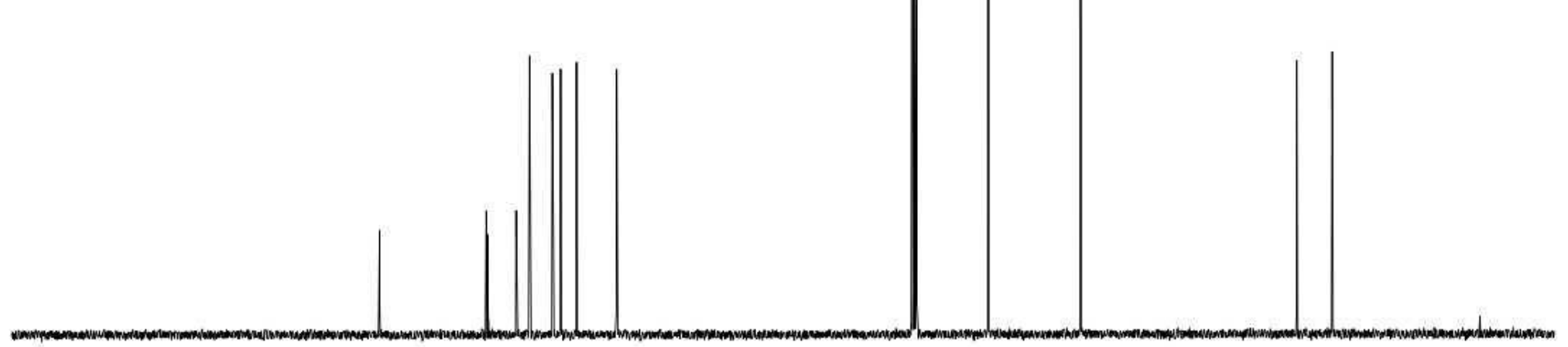

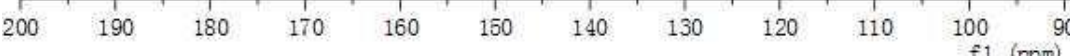

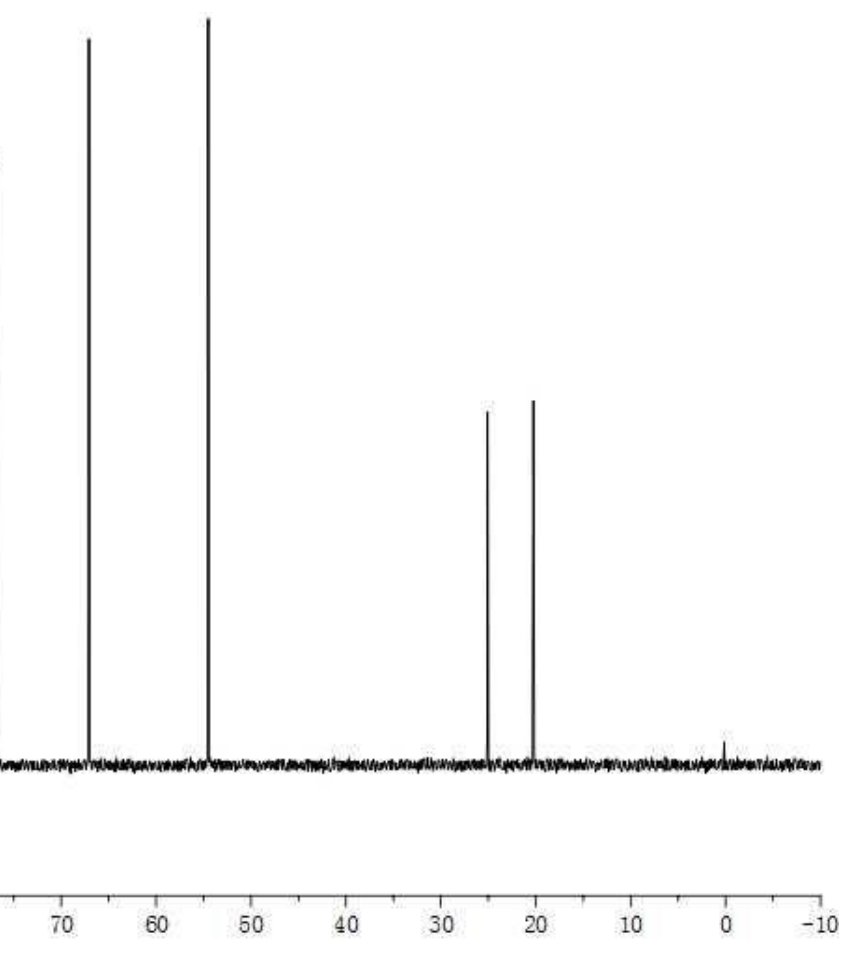




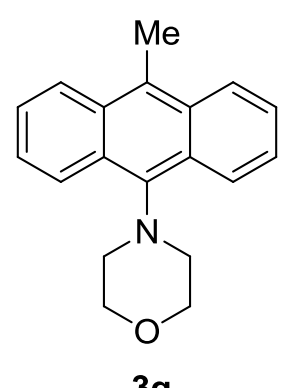

$3 \mathbf{g}$

${ }^{1} \mathrm{H} \mathrm{NMR}\left(400 \mathrm{MHz}, \mathrm{CDCl}_{3}\right)$

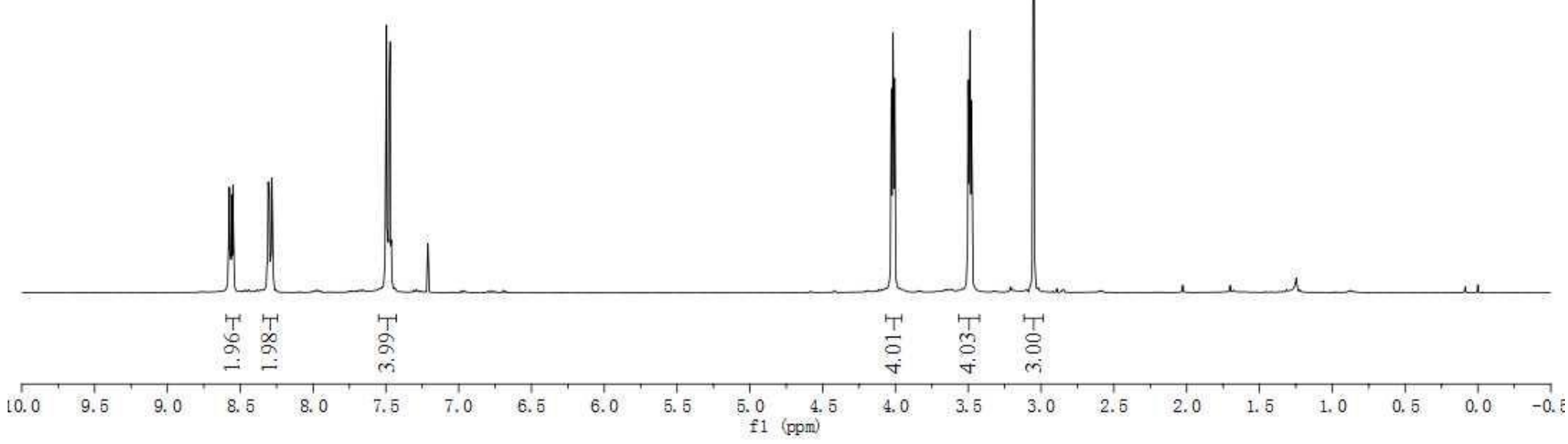



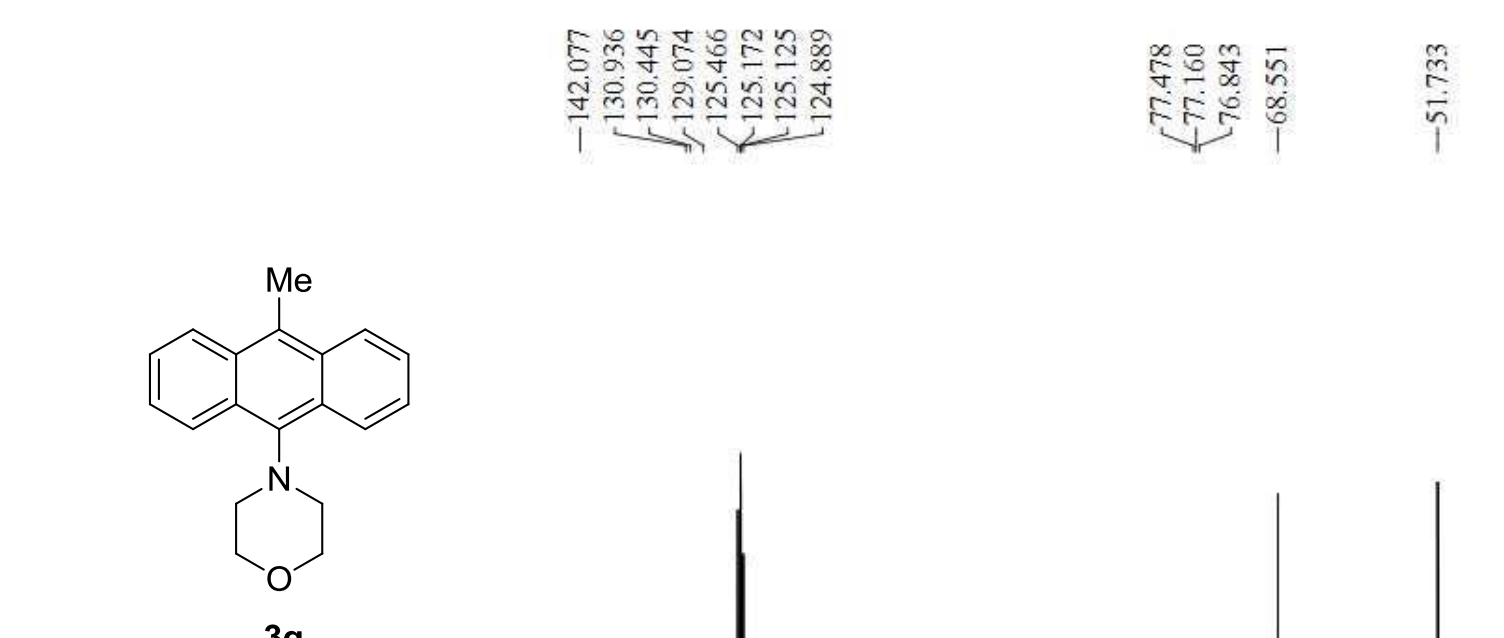

${ }^{13} \mathrm{C} \mathrm{NMR}\left(100 \mathrm{MHz}, \mathrm{CDCl}_{3}\right)$

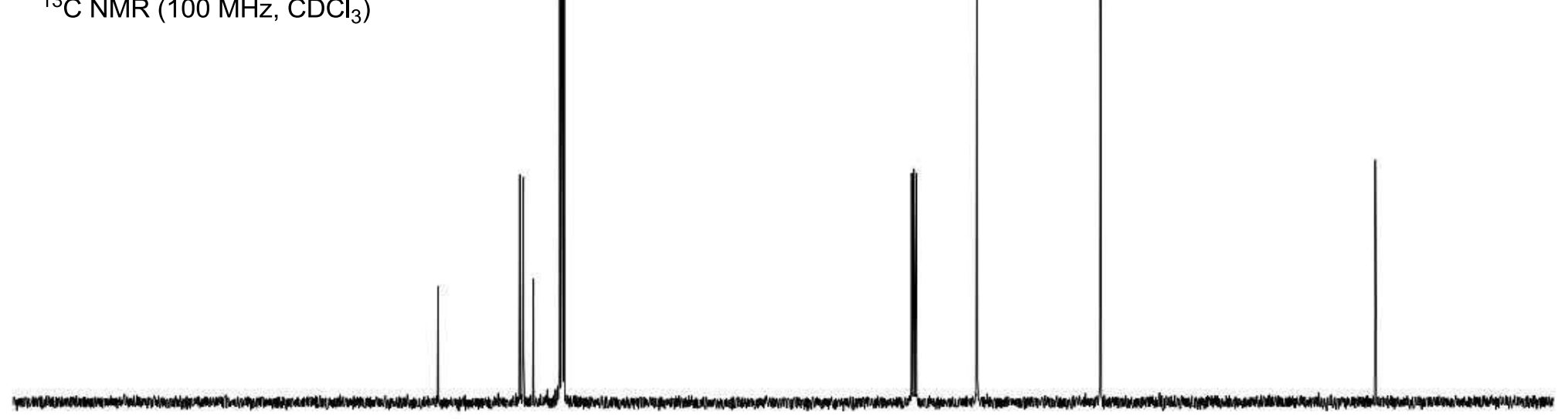




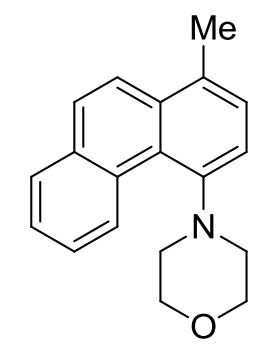

3h

${ }^{1} \mathrm{H} \mathrm{NMR}\left(400 \mathrm{MHz}, \mathrm{CDCl}_{3}\right)$

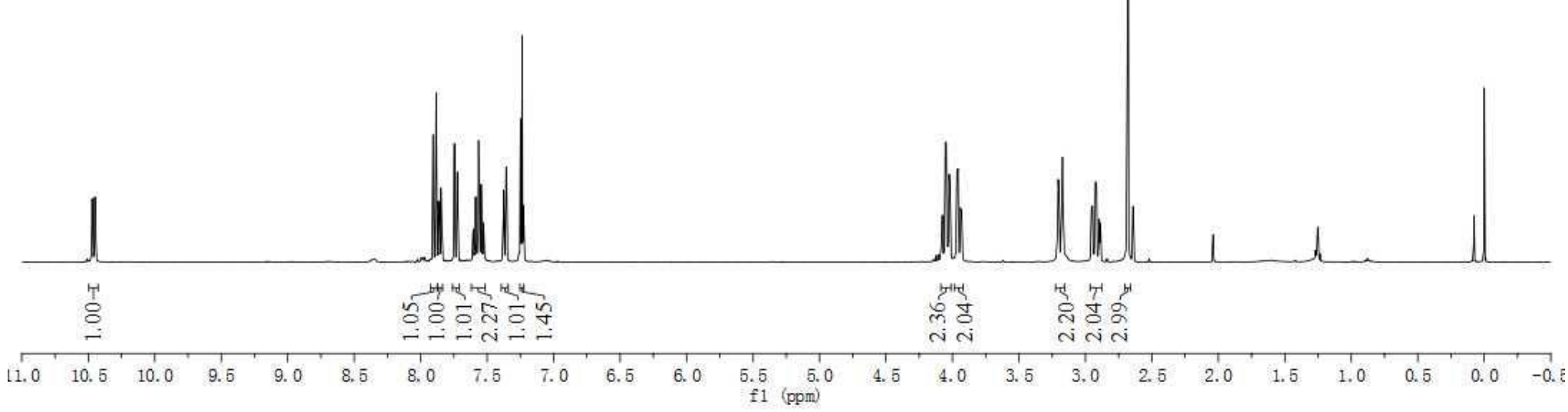

S102 


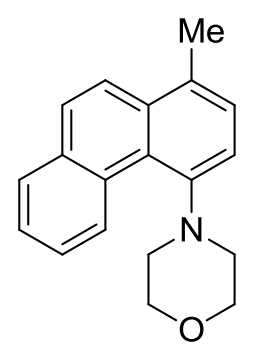

3h

${ }^{13} \mathrm{C}$ NMR $\left(100 \mathrm{MHz}, \mathrm{CDCl}_{3}\right)$

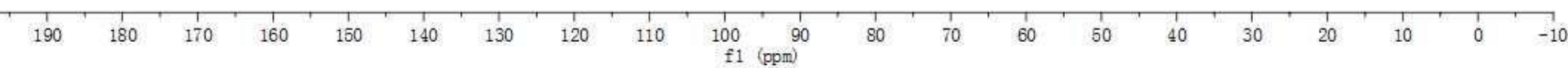

S103 


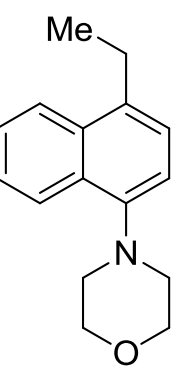

$3 \mathbf{i}$

${ }^{1} \mathrm{H} \mathrm{NMR}\left(400 \mathrm{MHz}, \mathrm{CDCl}_{3}\right)$

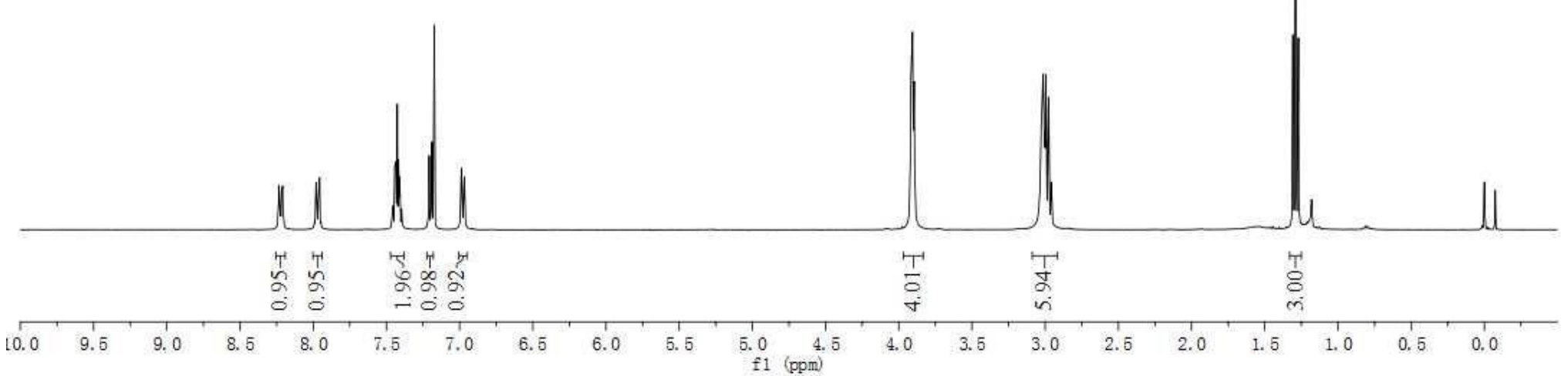

S104 


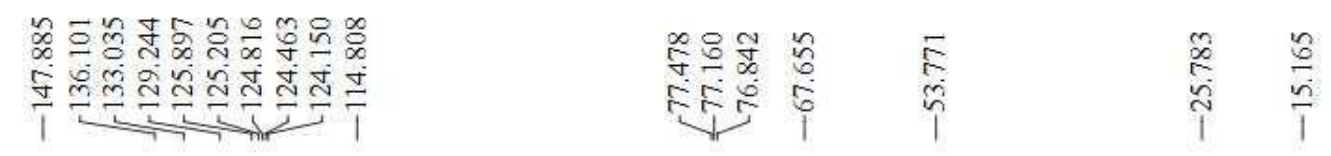
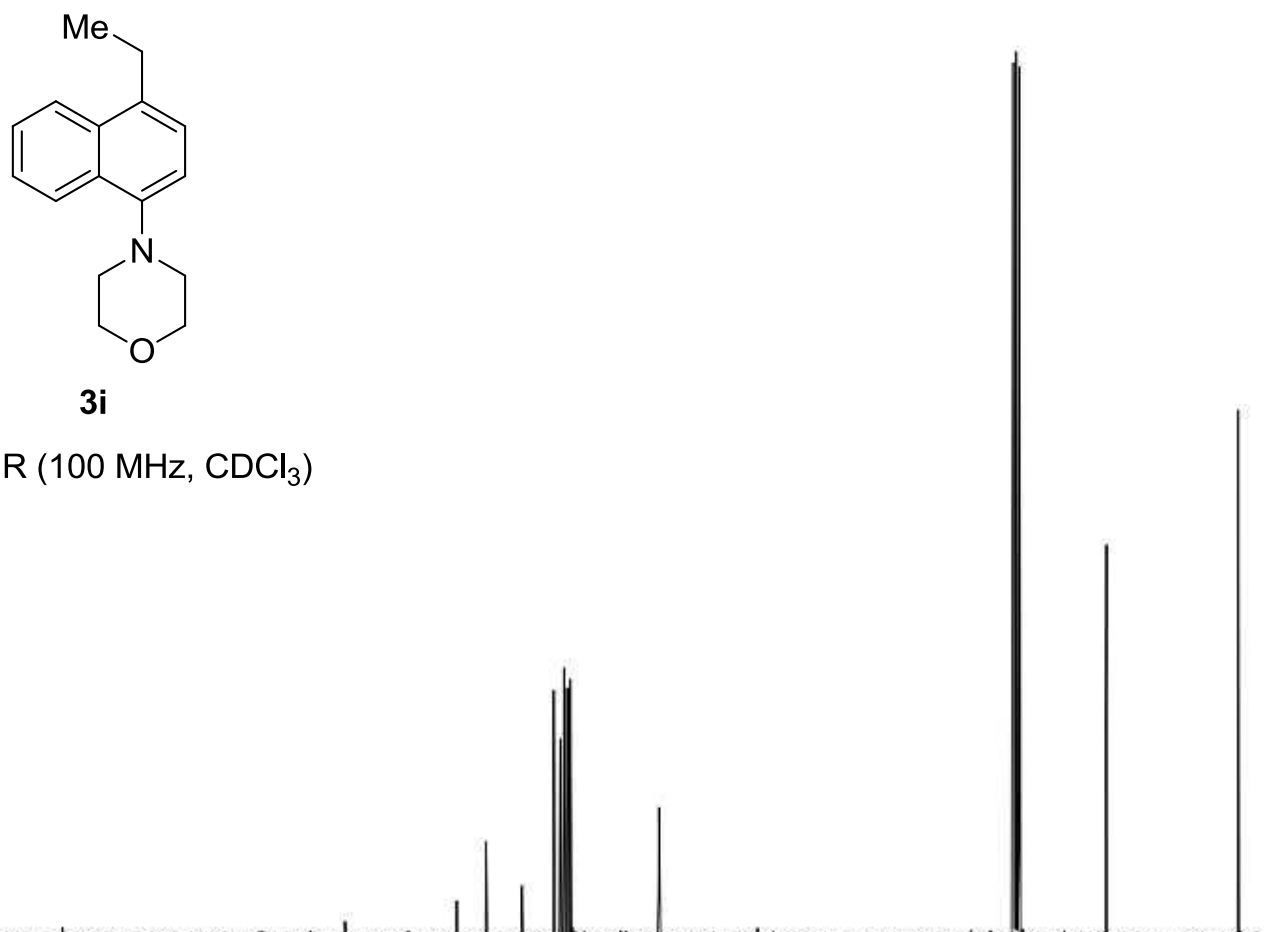

${ }^{13} \mathrm{C}$ NMR $\left(100 \mathrm{MHz}, \mathrm{CDCl}_{3}\right)$

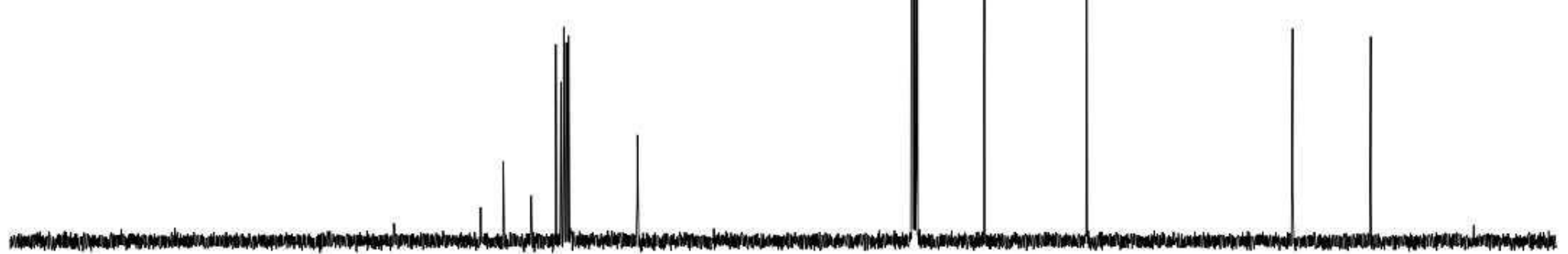

200

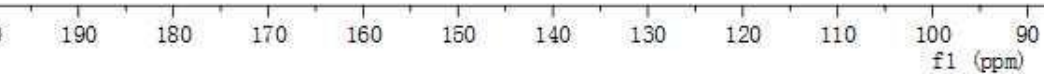

S105 


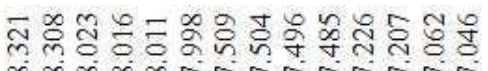

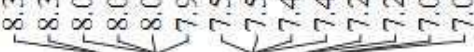

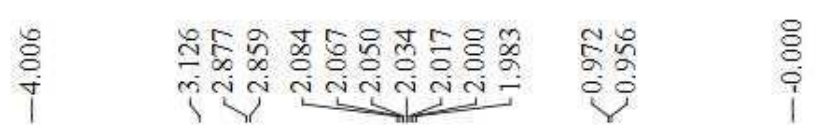

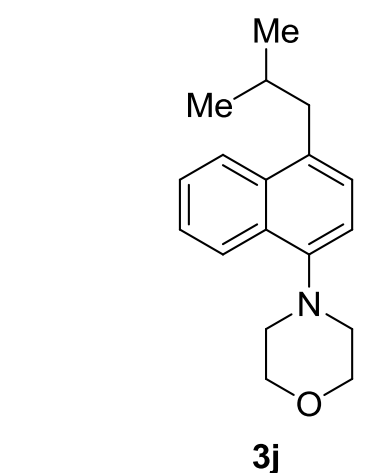

${ }^{1} \mathrm{H}$ NMR (400 MHz, $\mathrm{CDCl}_{3}$ )

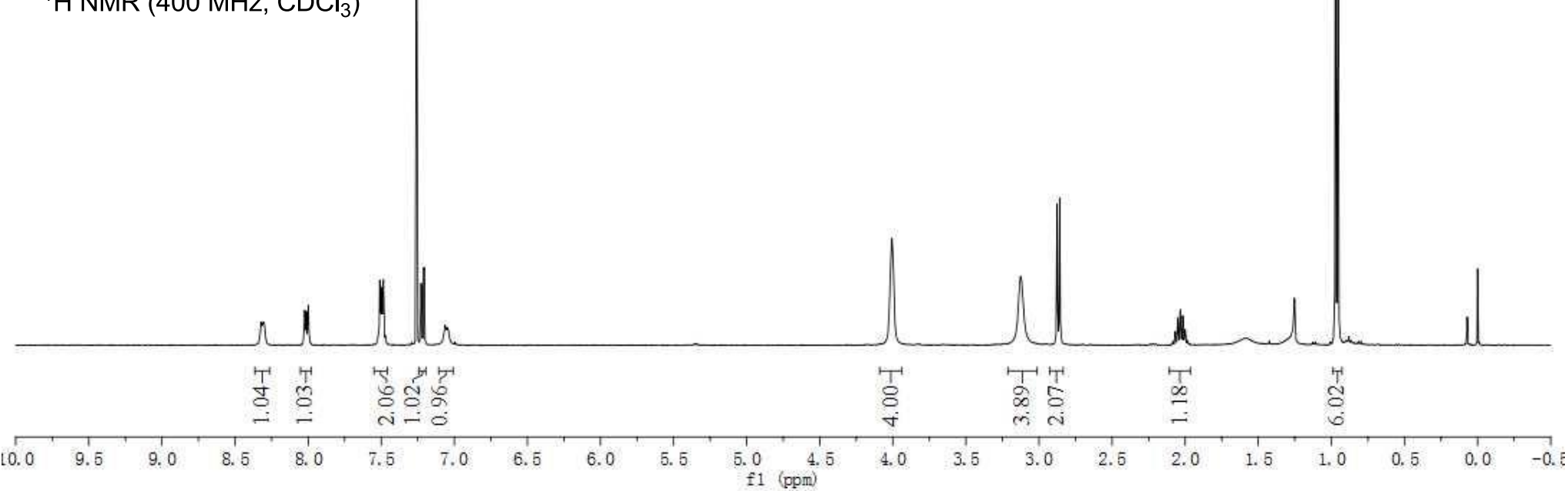

S106 

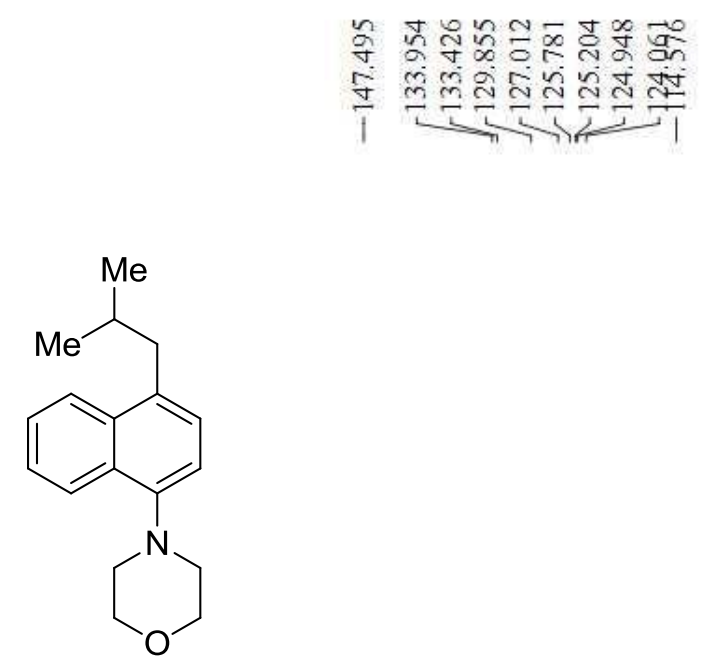

3j

${ }^{13} \mathrm{C} \mathrm{NMR}\left(100 \mathrm{MHz}, \mathrm{CDCl}_{3}\right)$ 


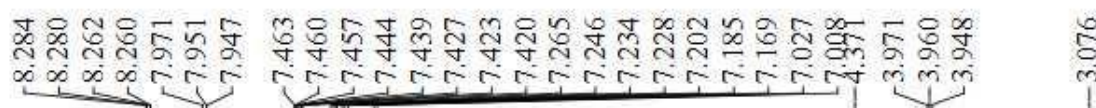

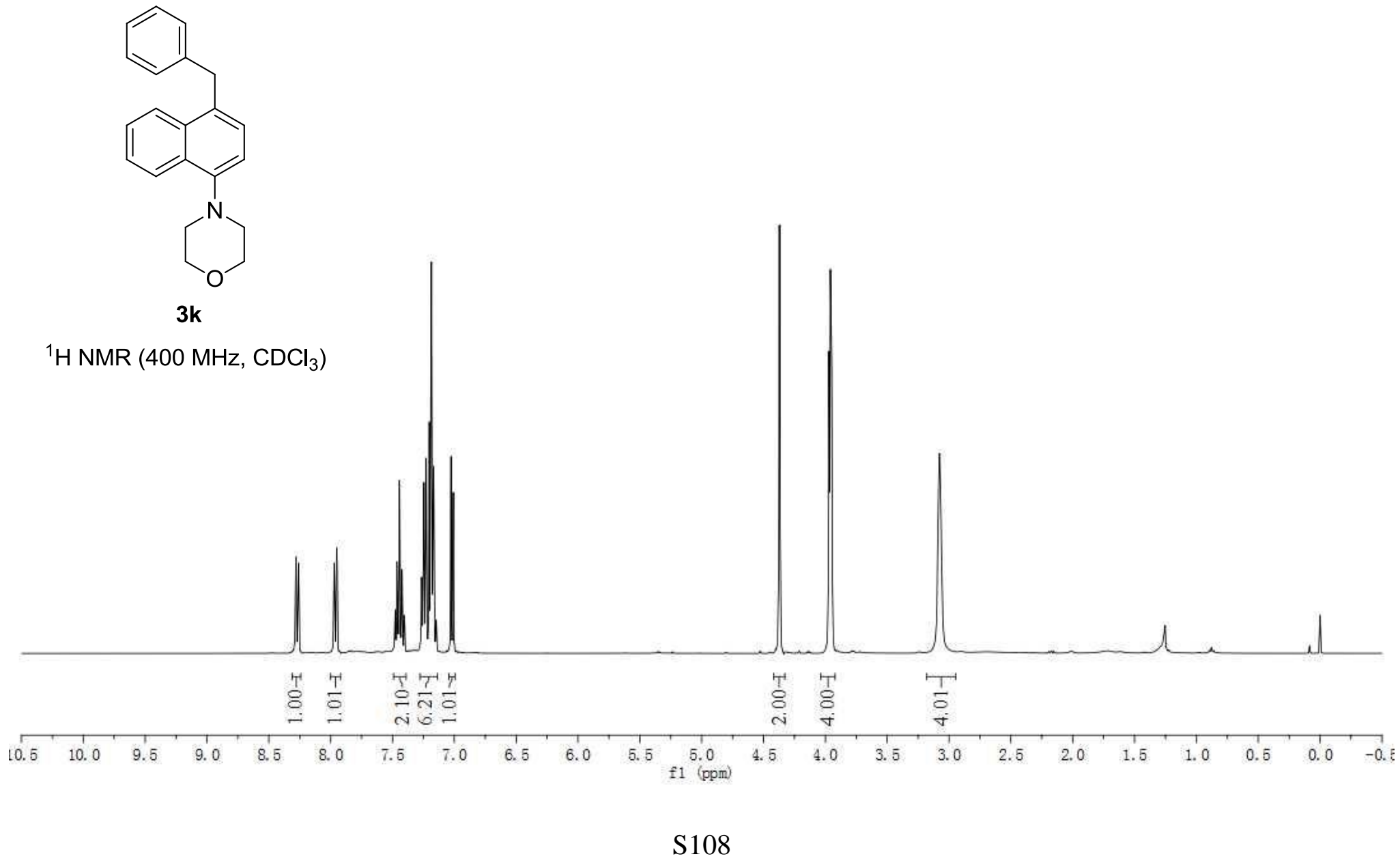




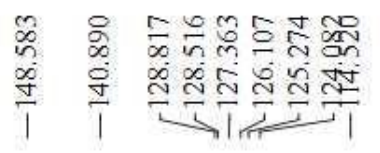

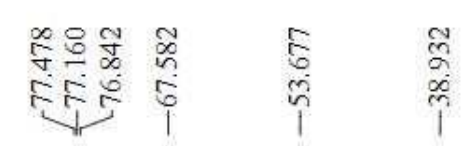

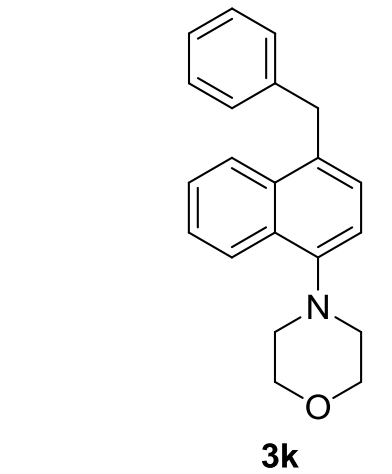

${ }^{13} \mathrm{C}$ NMR $\left(100 \mathrm{MHz}, \mathrm{CDCl}_{3}\right)$
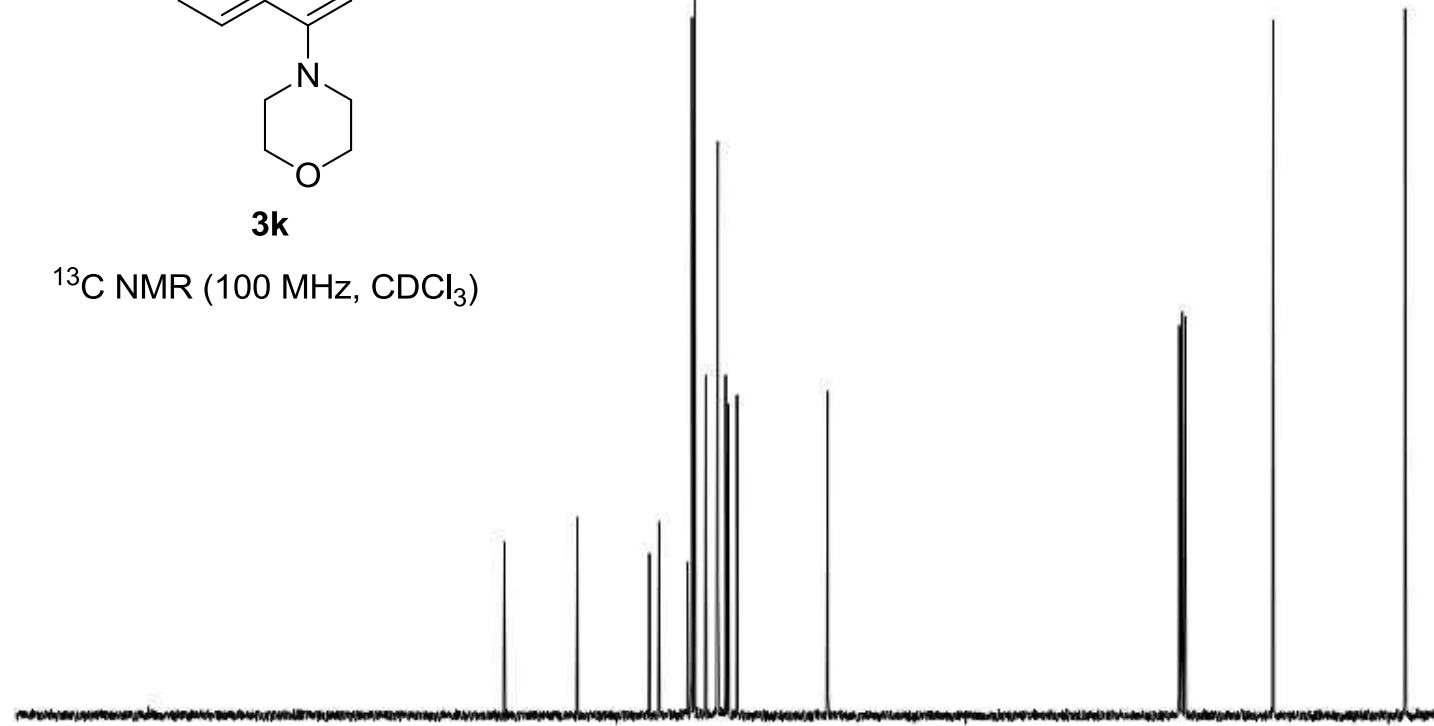

200

150

${ }_{130}^{1} 120$

$100 \quad 90$

80

1. 60

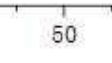

40 


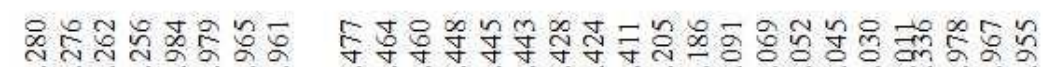
$\infty+\infty \infty \pi+\pi$

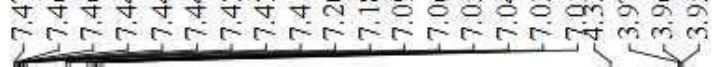

$\bar{g}$

$\stackrel{\text { กิ }}{\text { กิ }}$

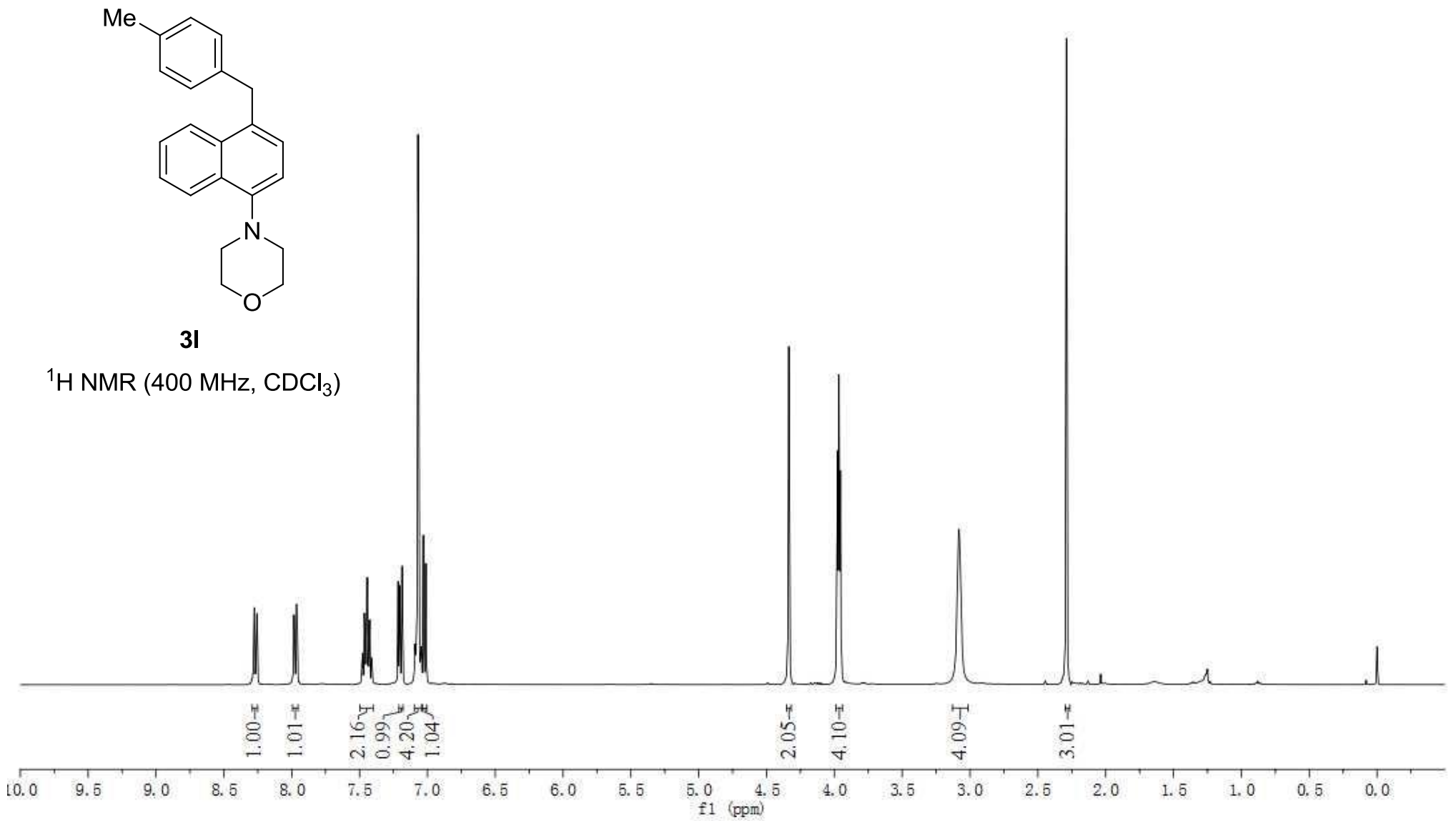




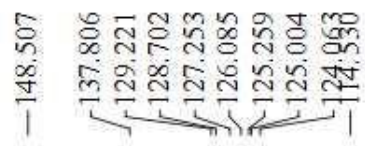

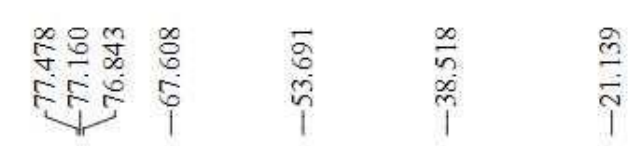

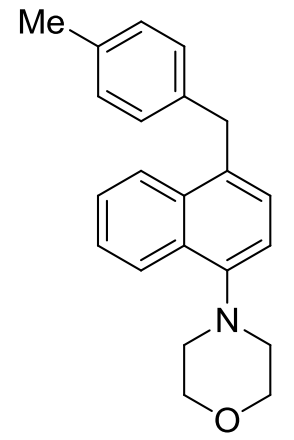

31

${ }^{13} \mathrm{C}$ NMR $\left(100 \mathrm{MHz}, \mathrm{CDCl}_{3}\right)$

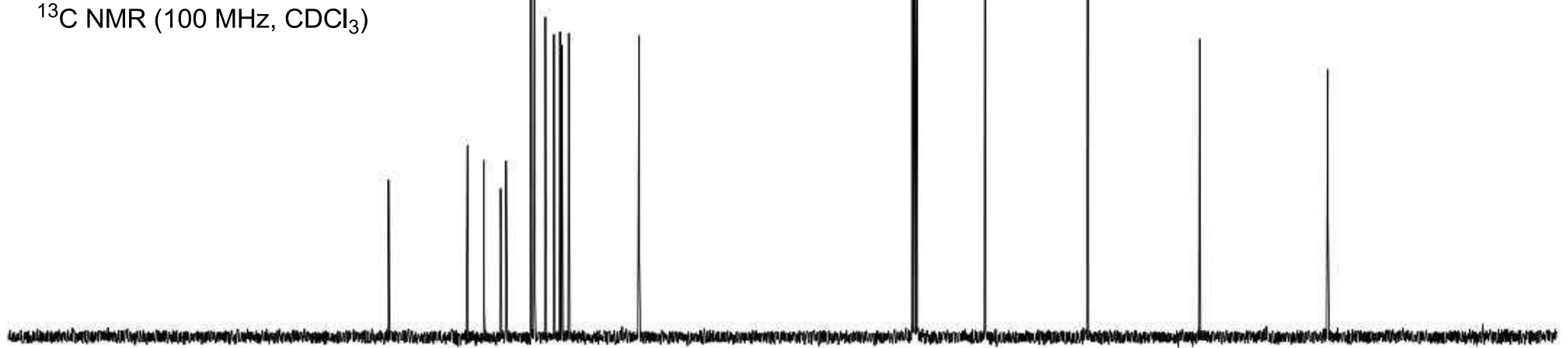

200

$170 \quad 160$

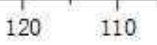

$100 \quad 90$

80

$60 \quad 50$

40

S111 


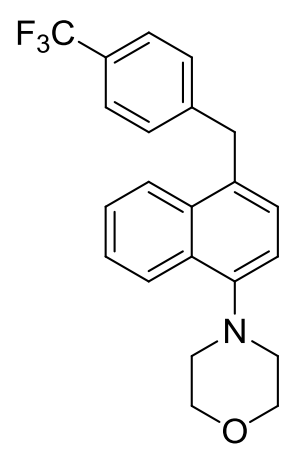

$3 \mathrm{~m}$

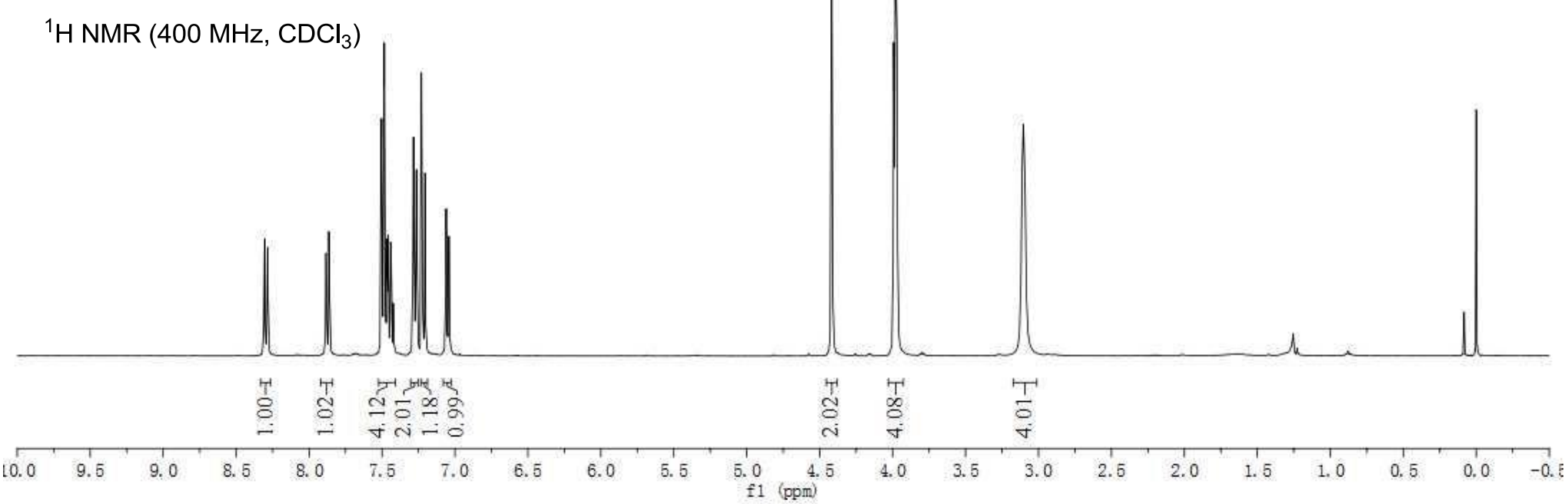

S112 

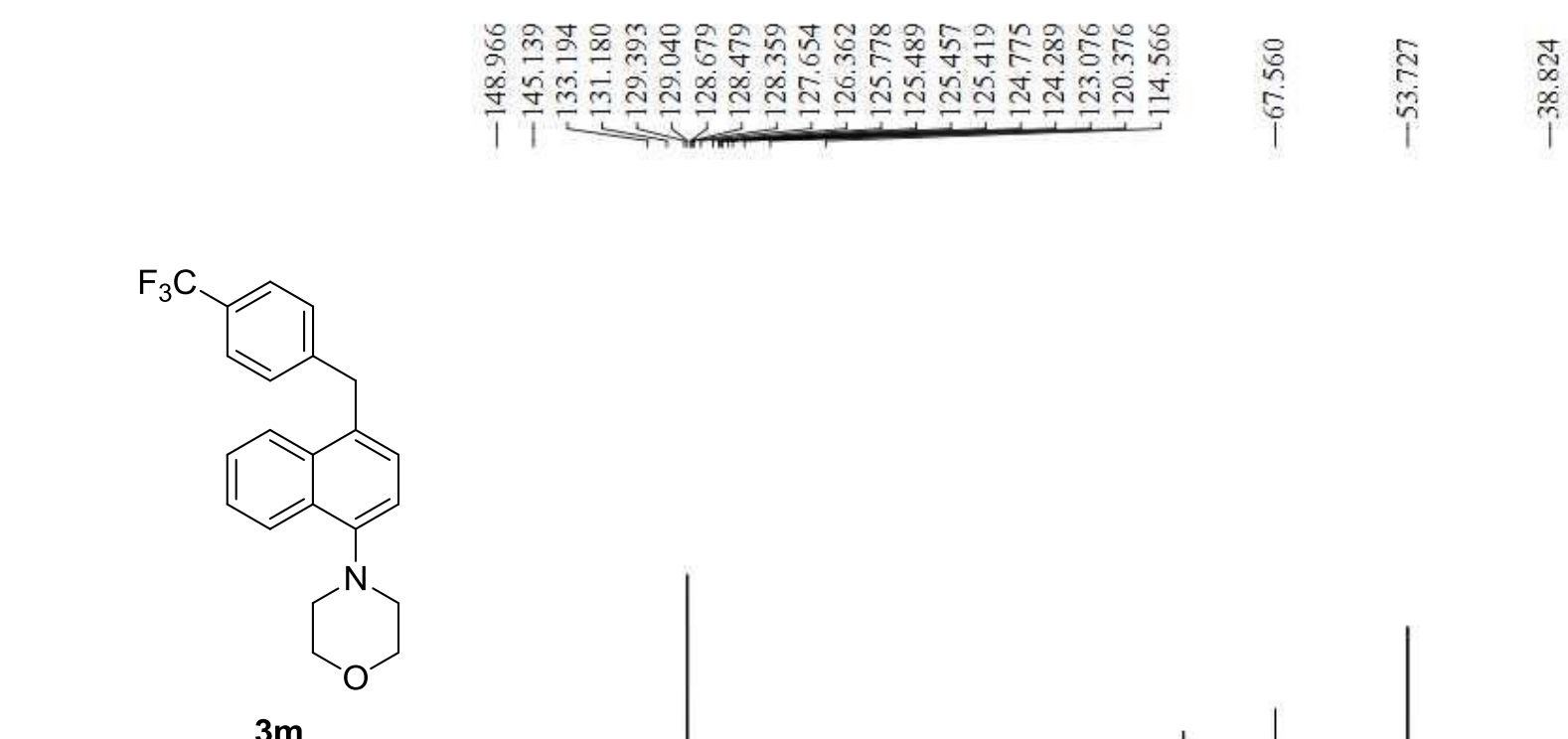

${ }^{13} \mathrm{C}$ NMR $\left(100 \mathrm{MHz}, \mathrm{CDCl}_{3}\right)$
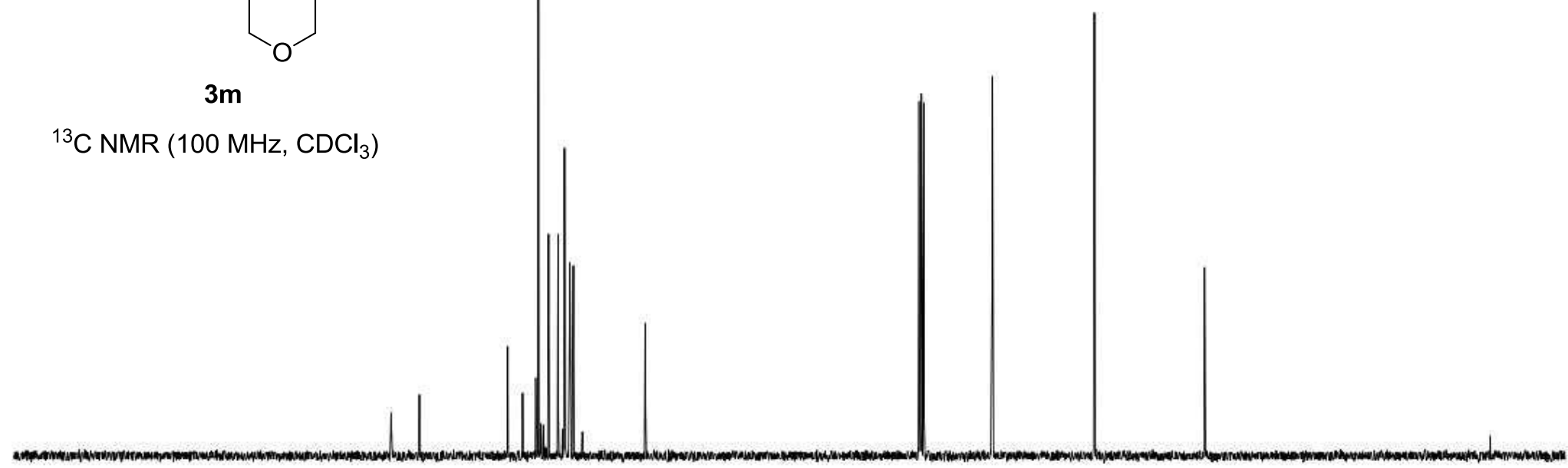

200

\begin{tabular}{|c|c|c|c|c|c|c|c|c|c|c|}
\hline 190 & 18 & & 170 & 160 & 150 & 14 & 13 & 12 & 110 & ${ }^{100} 90$ \\
\hline
\end{tabular}




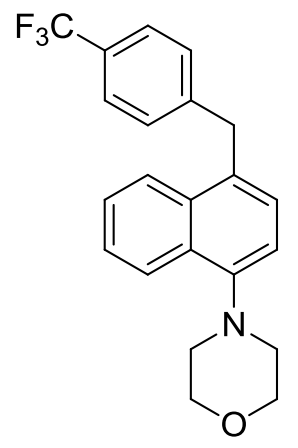

$3 m$

${ }^{19} \mathrm{~F} \mathrm{NMR}\left(376 \mathrm{MHz}, \mathrm{CDCl}_{3}\right)$

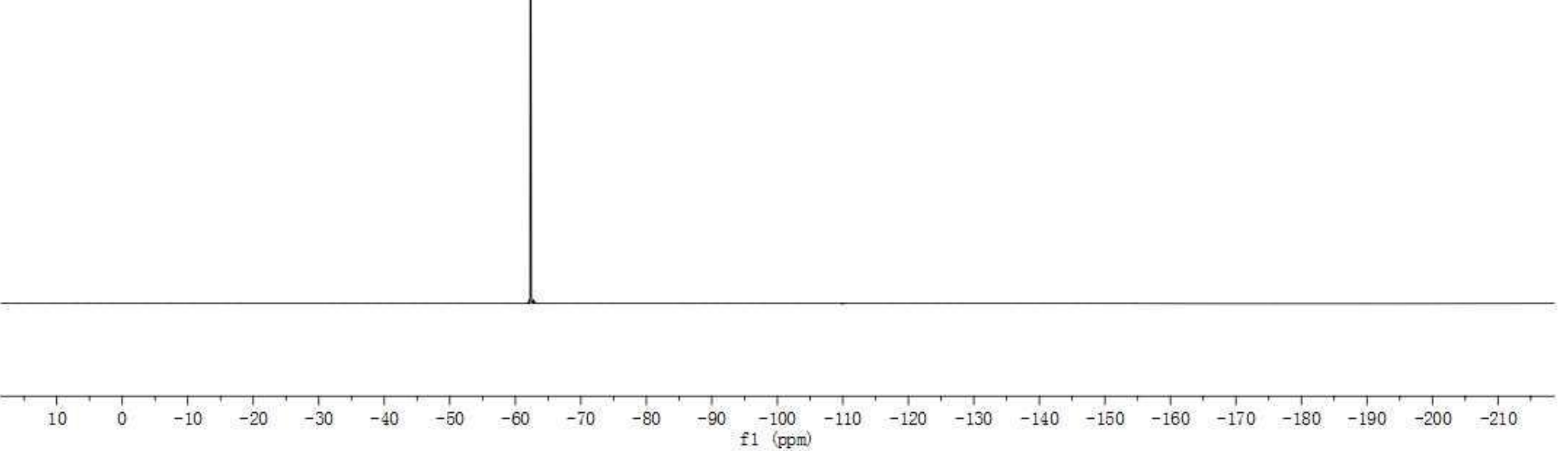




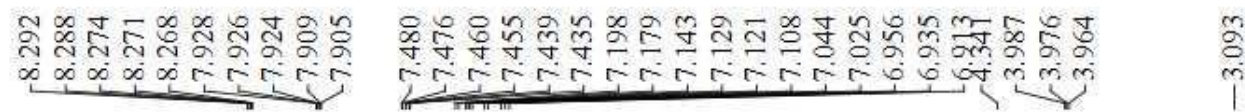

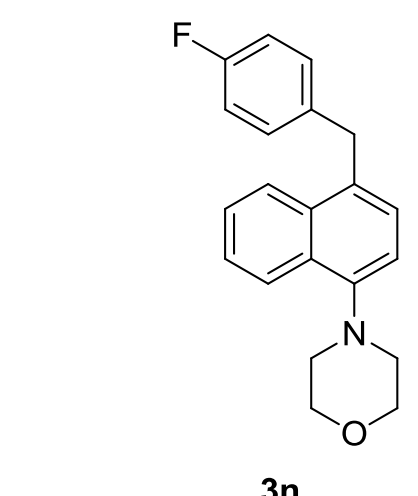

${ }^{1} \mathrm{HNMR}\left(400 \mathrm{MHz}, \mathrm{CDCl}_{3}\right)$

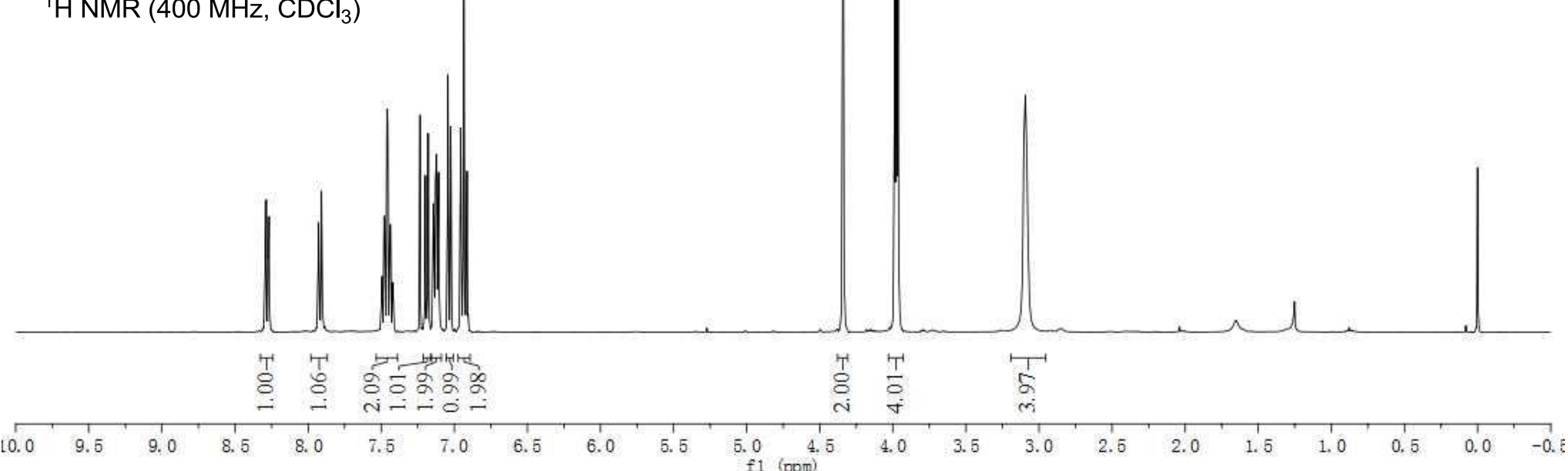

S115 


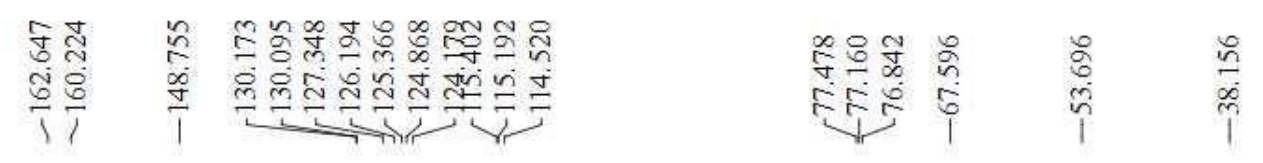

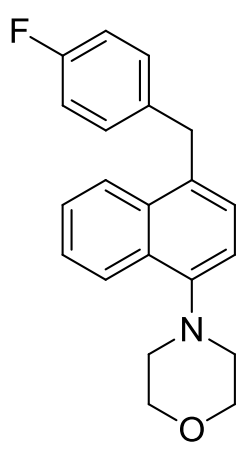

$3 n$

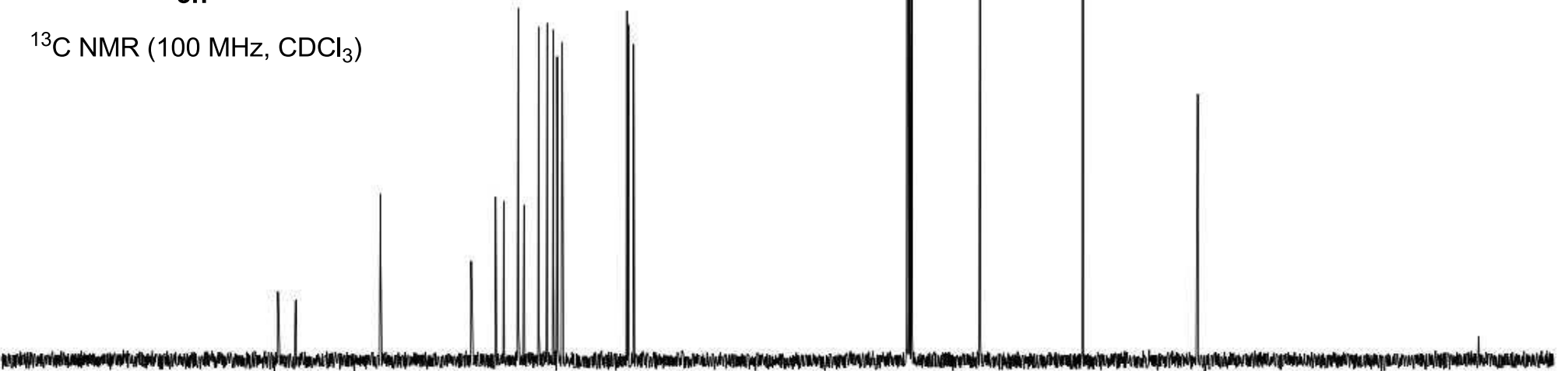

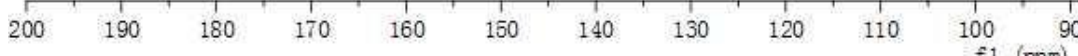

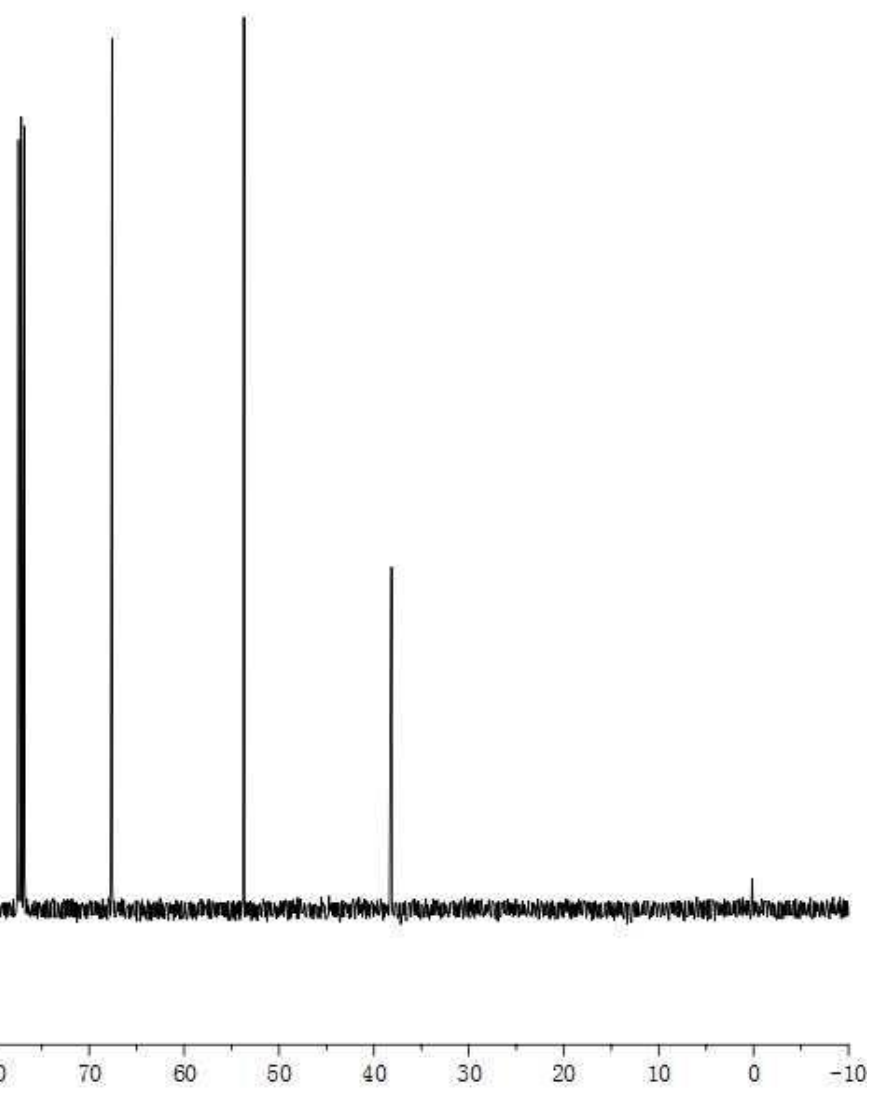

S116 
$\frac{\overline{7}}{\frac{7}{\bar{i}}}$

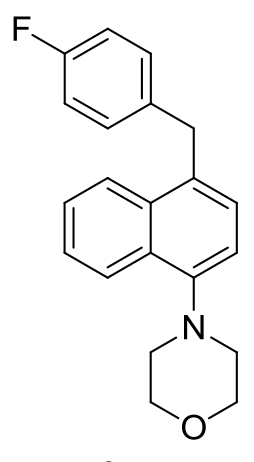

$3 n$

${ }^{19} \mathrm{~F}$ NMR $\left(376 \mathrm{MHz}, \mathrm{CDCl}_{3}\right)$

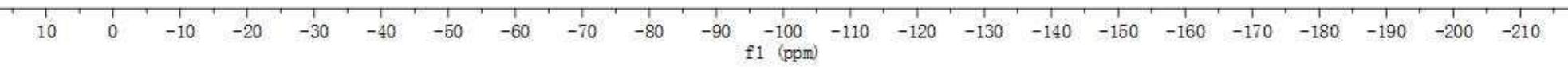




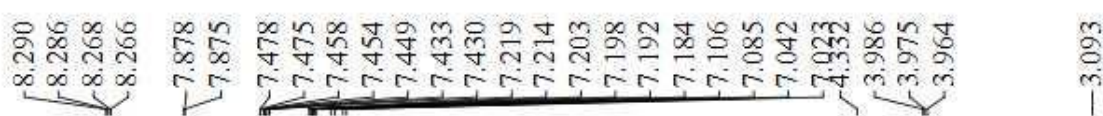

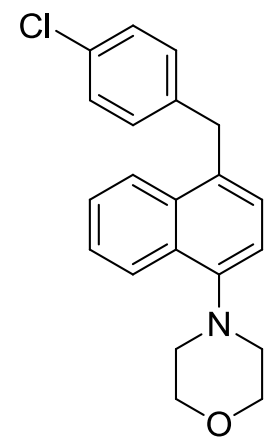

30

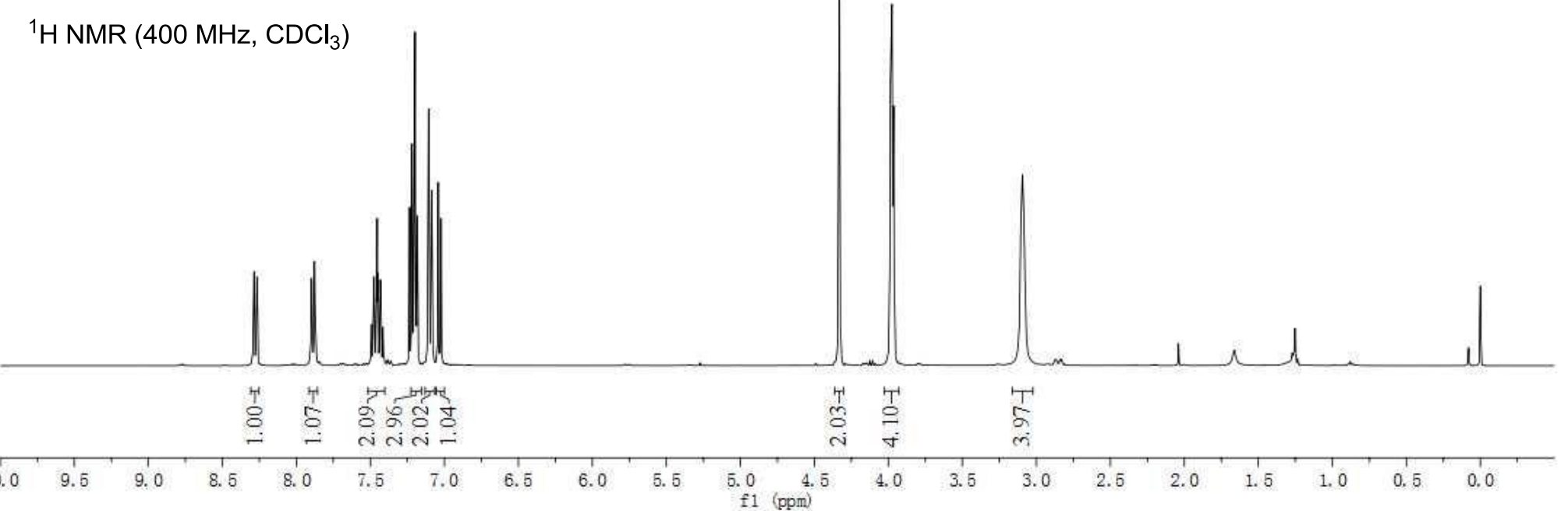

S118 


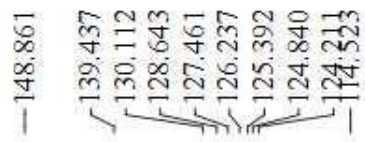

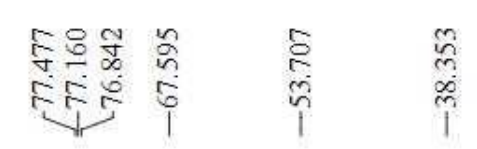

$\mathrm{Cl}$

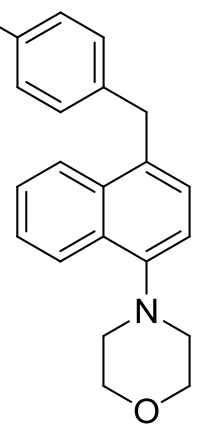

30

${ }^{13} \mathrm{C}$ NMR $\left(100 \mathrm{MHz}, \mathrm{CDCl}_{3}\right)$

$200 \quad 190$

140

1
120

$100 \quad 90$

80

1. 60

50

40

S119 


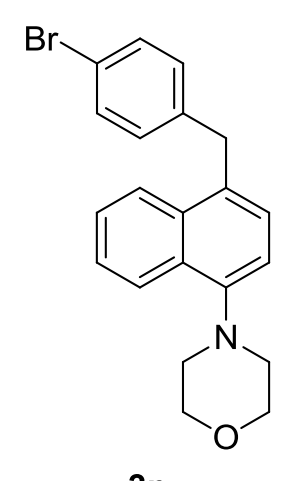

${ }^{1} \mathrm{H}$ NMR $\left(400 \mathrm{MHz}, \mathrm{CDCl}_{3}\right)$

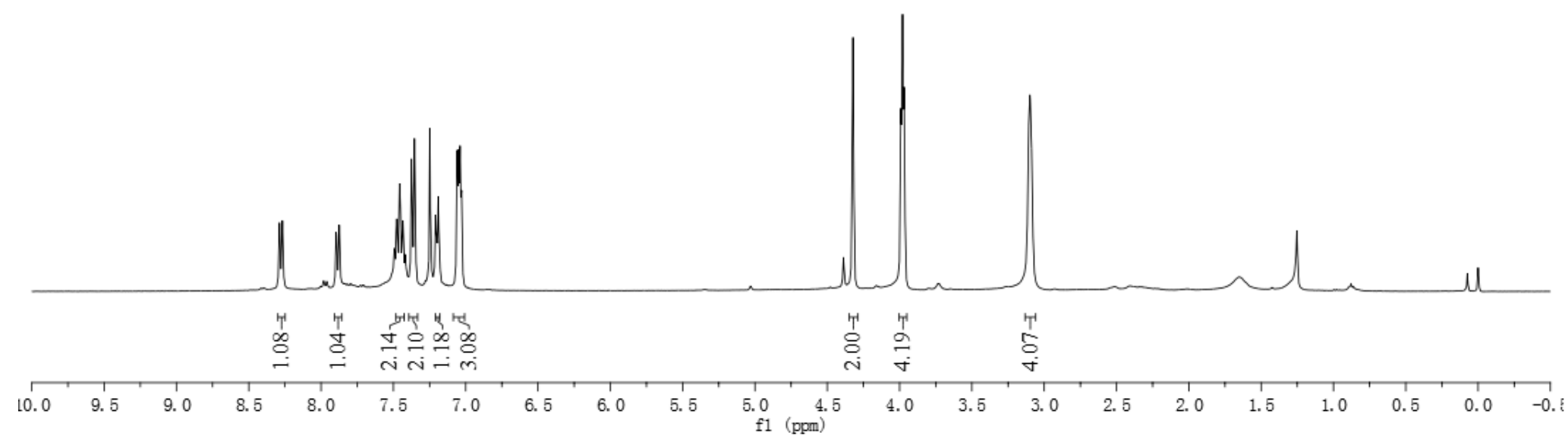



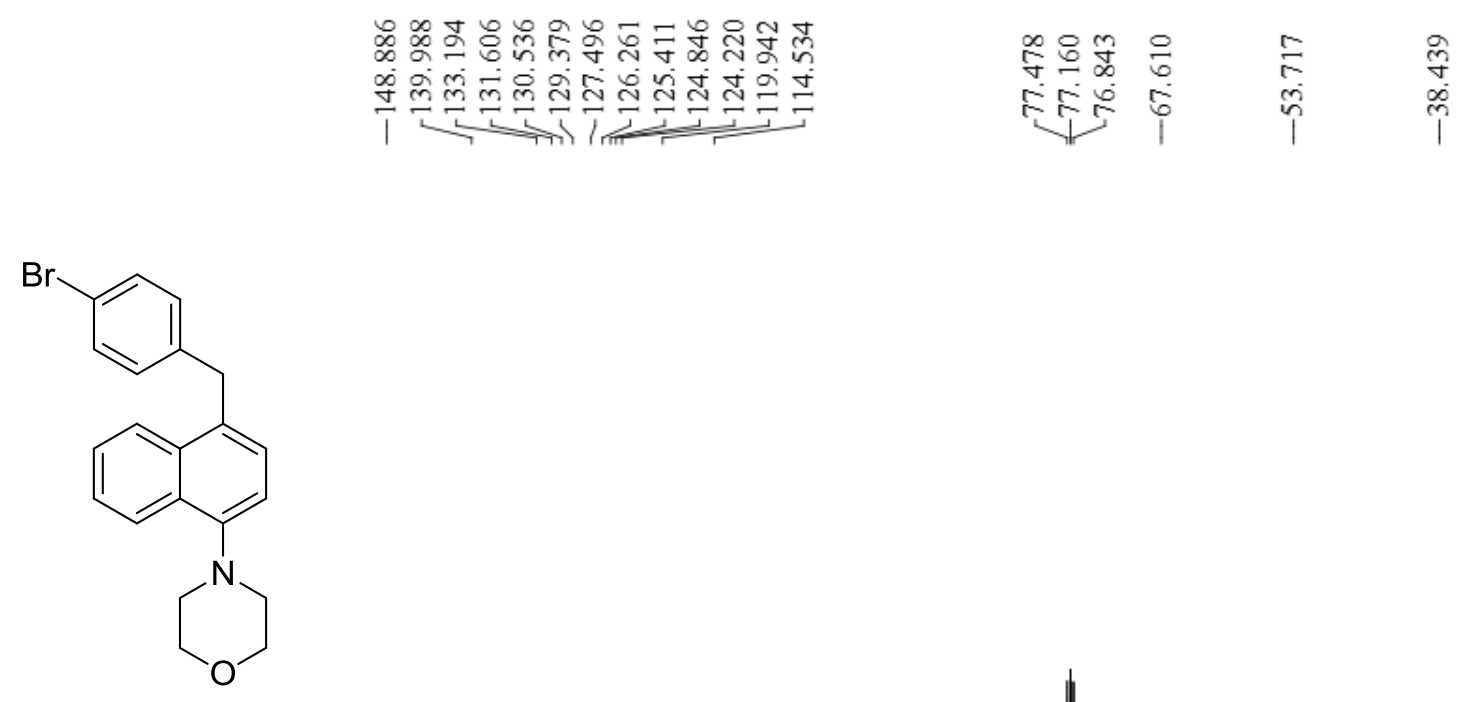

$3 p$

${ }^{13} \mathrm{C}$ NMR $\left(100 \mathrm{MHz}, \mathrm{CDCl}_{3}\right)$

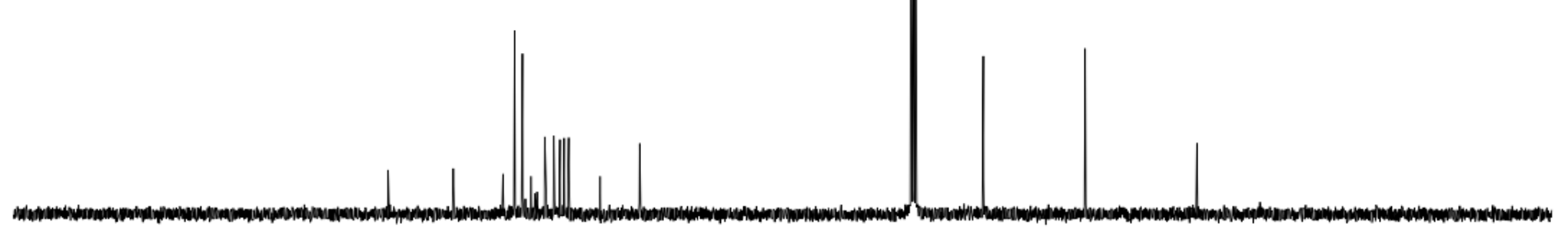

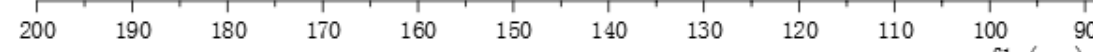



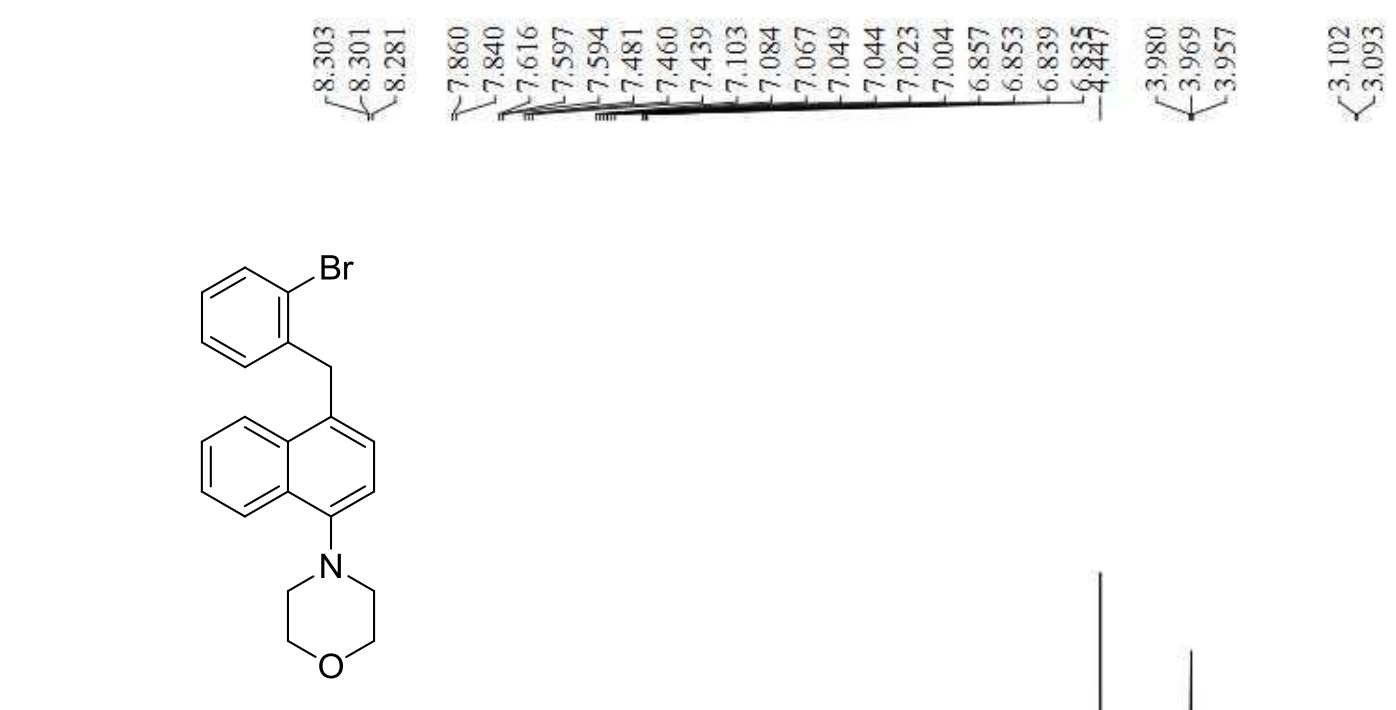

$3 q$

${ }^{1} \mathrm{H}$ NMR $\left(400 \mathrm{MHz}, \mathrm{CDCl}_{3}\right)$

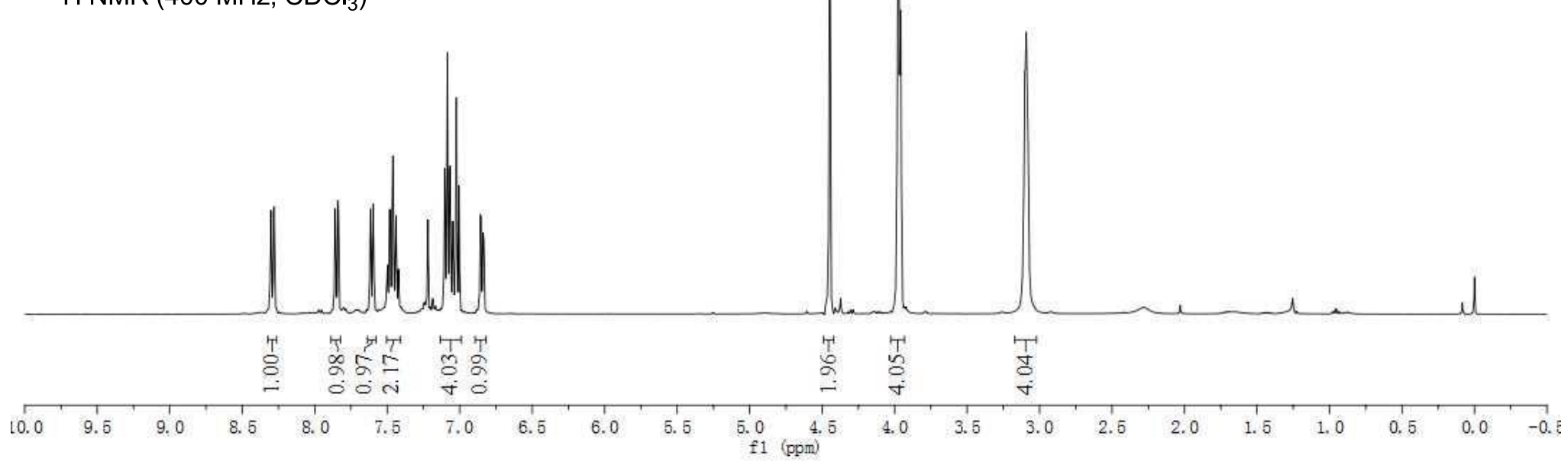

S122 


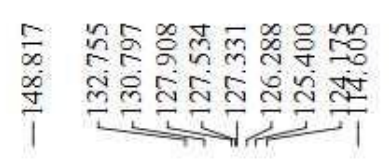

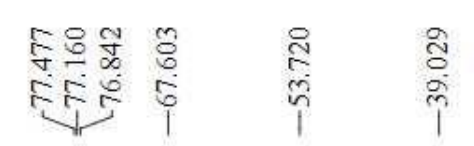

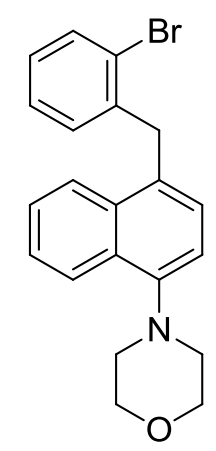

$3 q$

${ }^{13} \mathrm{C} \mathrm{NMR} \mathrm{(100} \mathrm{MHz,} \mathrm{CDCl}_{3}$ )

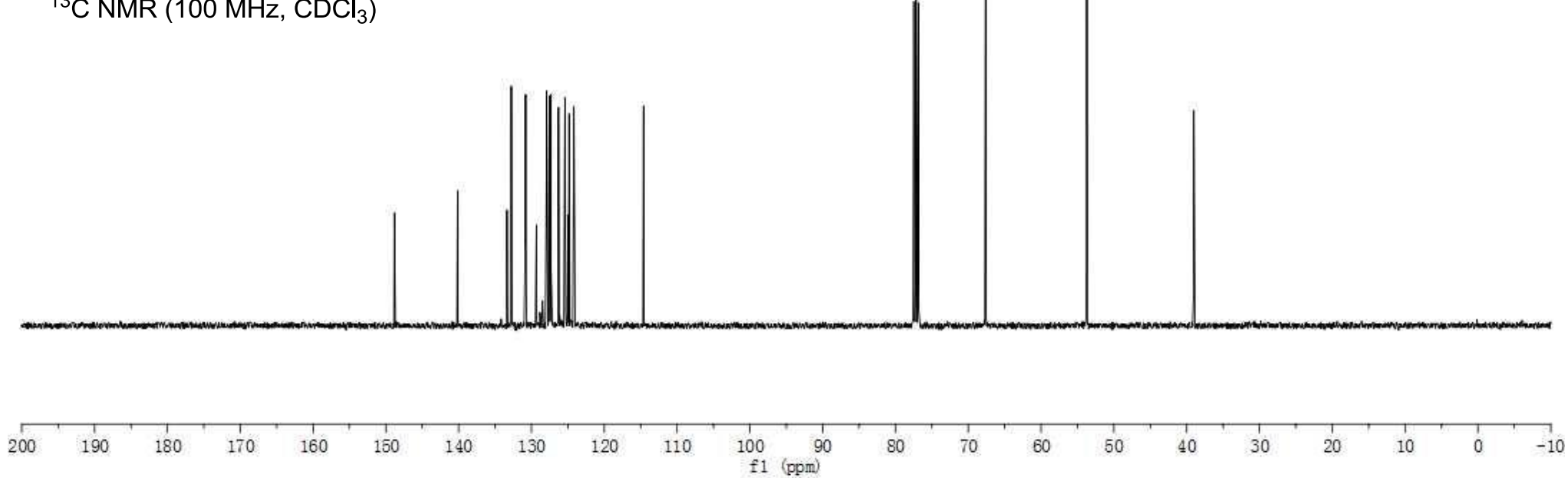

S123 


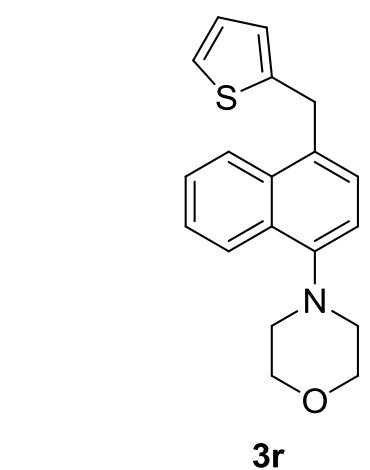

${ }^{1} \mathrm{H}$ NMR $\left(400 \mathrm{MHz}, \mathrm{CDCl}_{3}\right)$

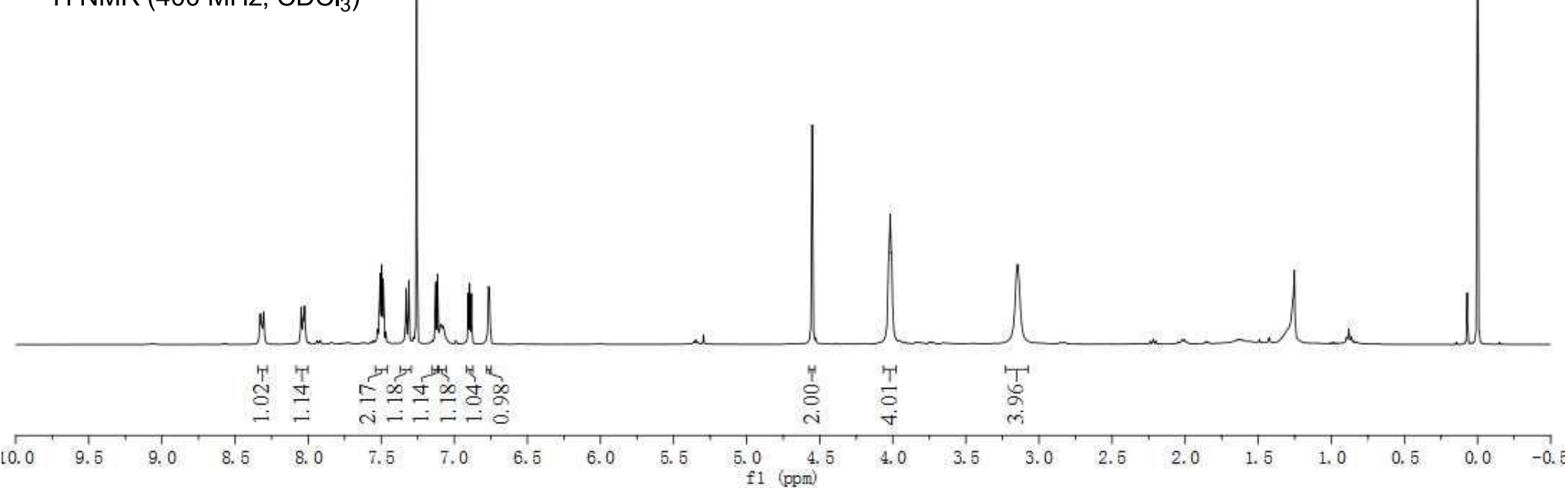




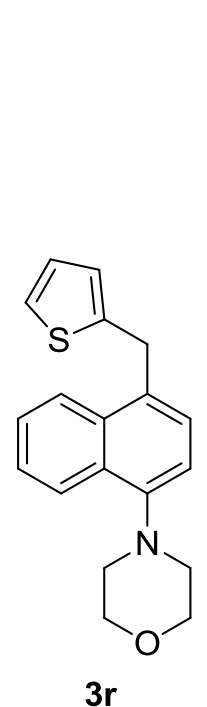

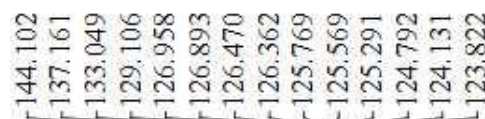

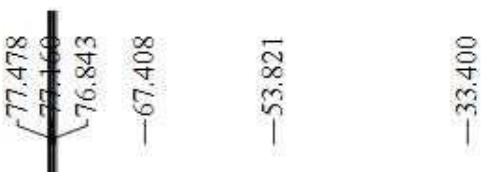

${ }^{13} \mathrm{C}$ NMR $\left(100 \mathrm{MHz}, \mathrm{CDCl}_{3}\right)$

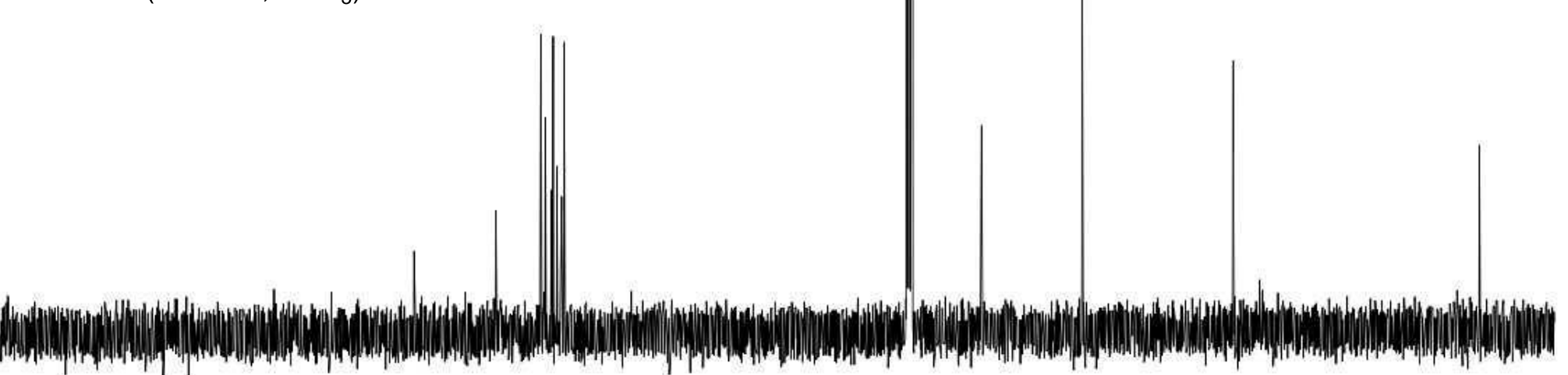

200

${ }_{170} \quad 160$

$150 \quad 140$

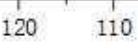

$100 \quad 90$
$\mathrm{f1}(\mathrm{ppm})$

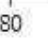

10. 60

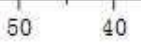

30

S125 


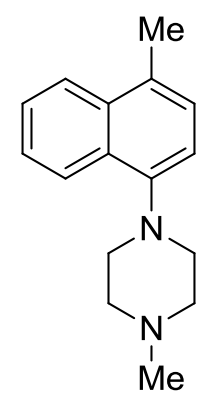

3s

${ }^{1} \mathrm{H} \mathrm{NMR}\left(400 \mathrm{MHz}, \mathrm{CDCl}_{3}\right)$

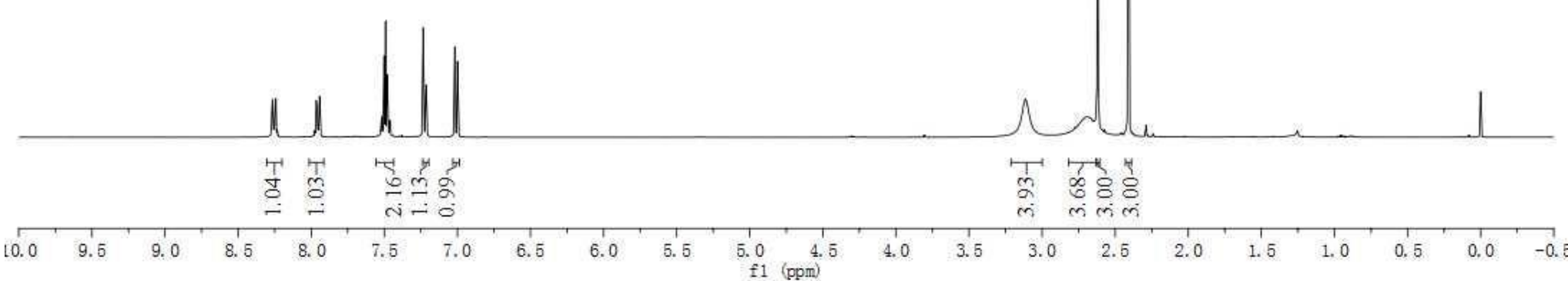



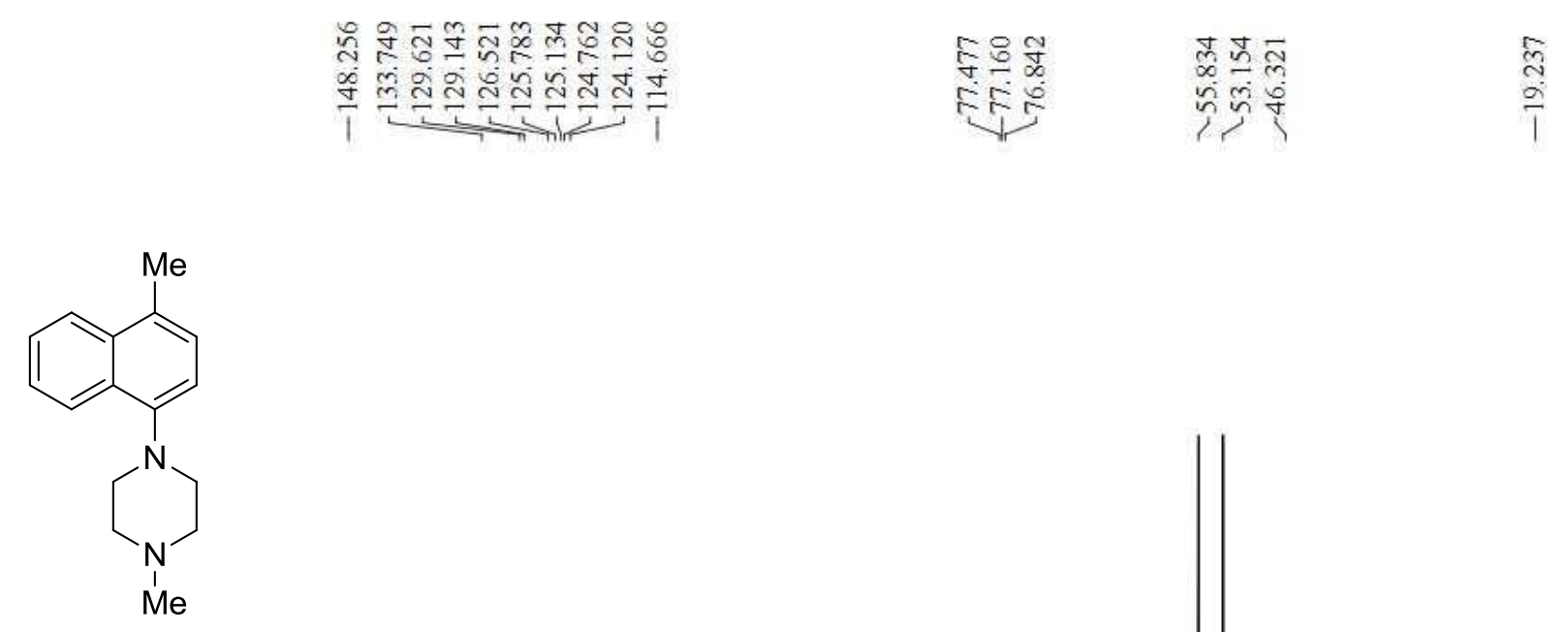

3s

${ }^{13} \mathrm{C}$ NMR $\left(100 \mathrm{MHz}, \mathrm{CDCl}_{3}\right)$

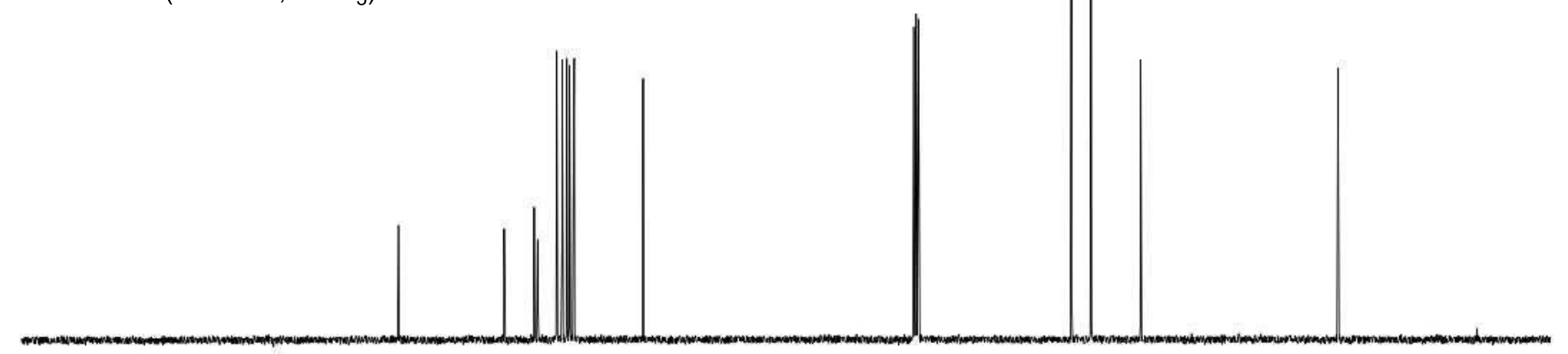

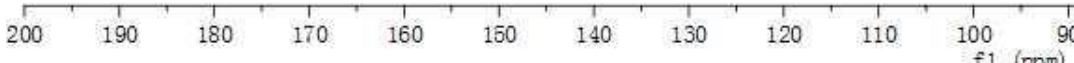




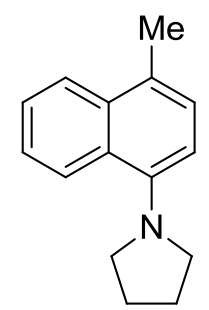

3t

${ }^{1} \mathrm{H} \mathrm{NMR}\left(400 \mathrm{MHz}, \mathrm{CDCl}_{3}\right)$

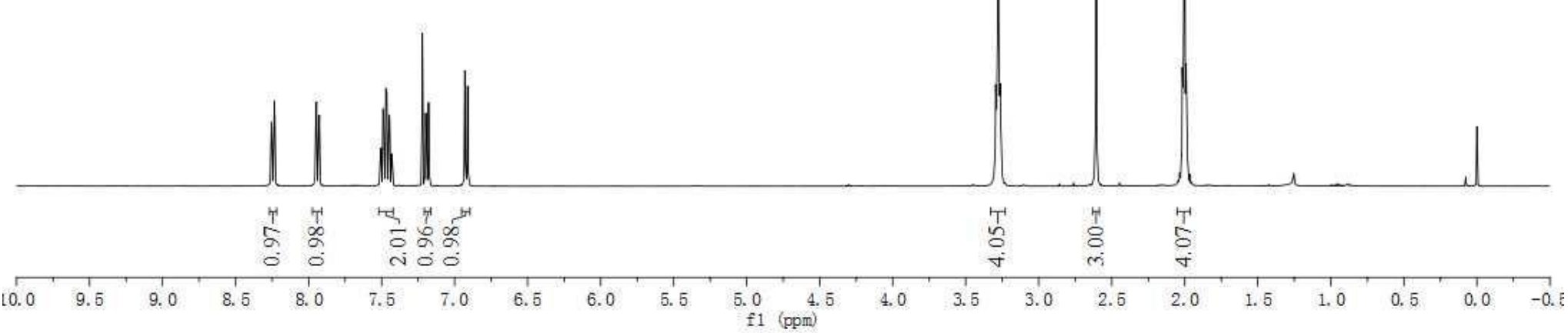

S128 

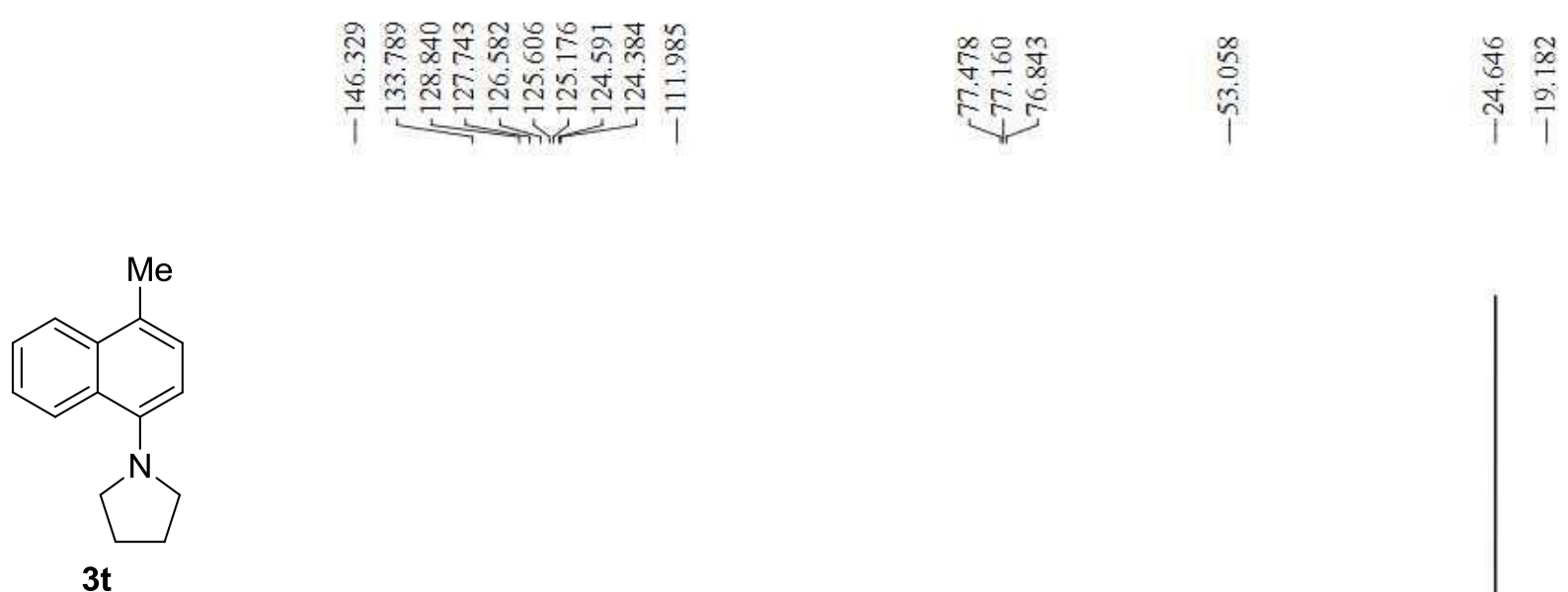

${ }^{13} \mathrm{C}$ NMR (100 MHz, $\mathrm{CDCl}_{3}$ )

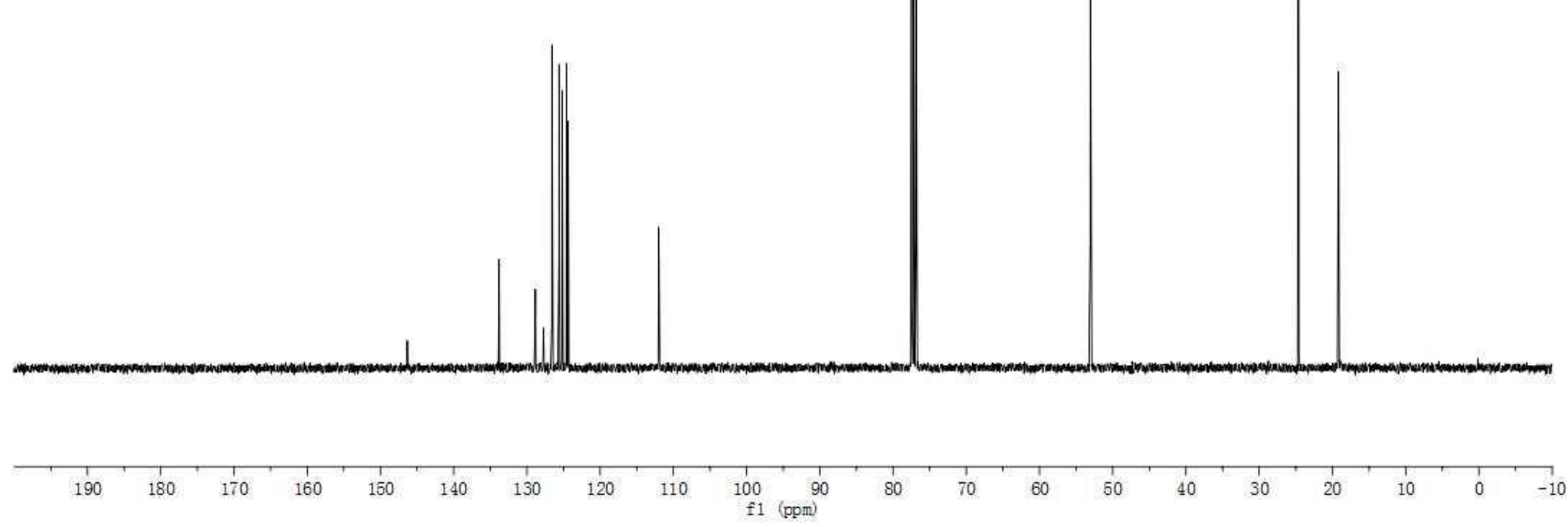




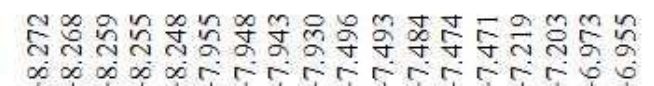

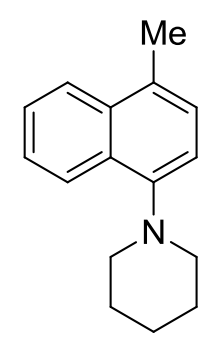

3u

${ }^{1} \mathrm{H}$ NMR (400 MHz, $\mathrm{CDCl}_{3}$ )
ㅎํำ

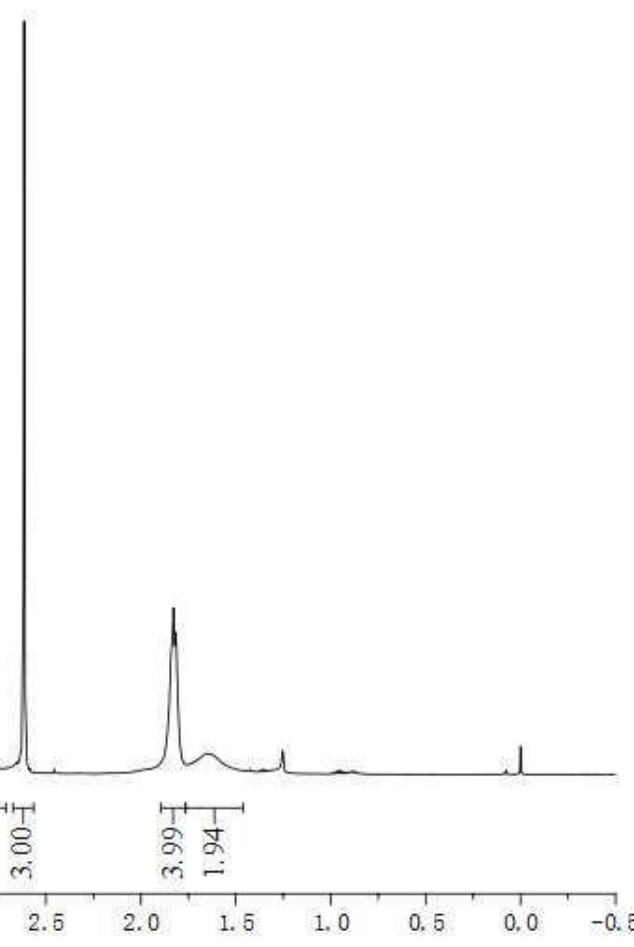



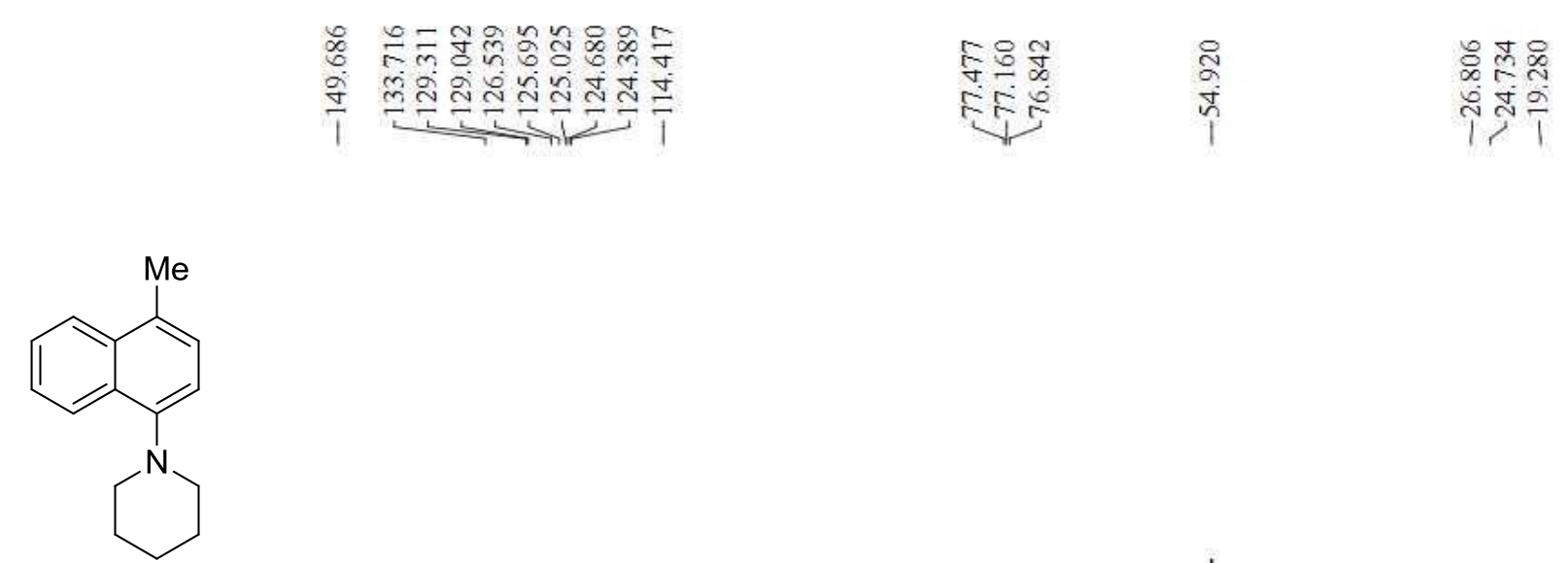

3u

${ }^{13} \mathrm{C} \mathrm{NMR}\left(100 \mathrm{MHz}, \mathrm{CDCl}_{3}\right)$

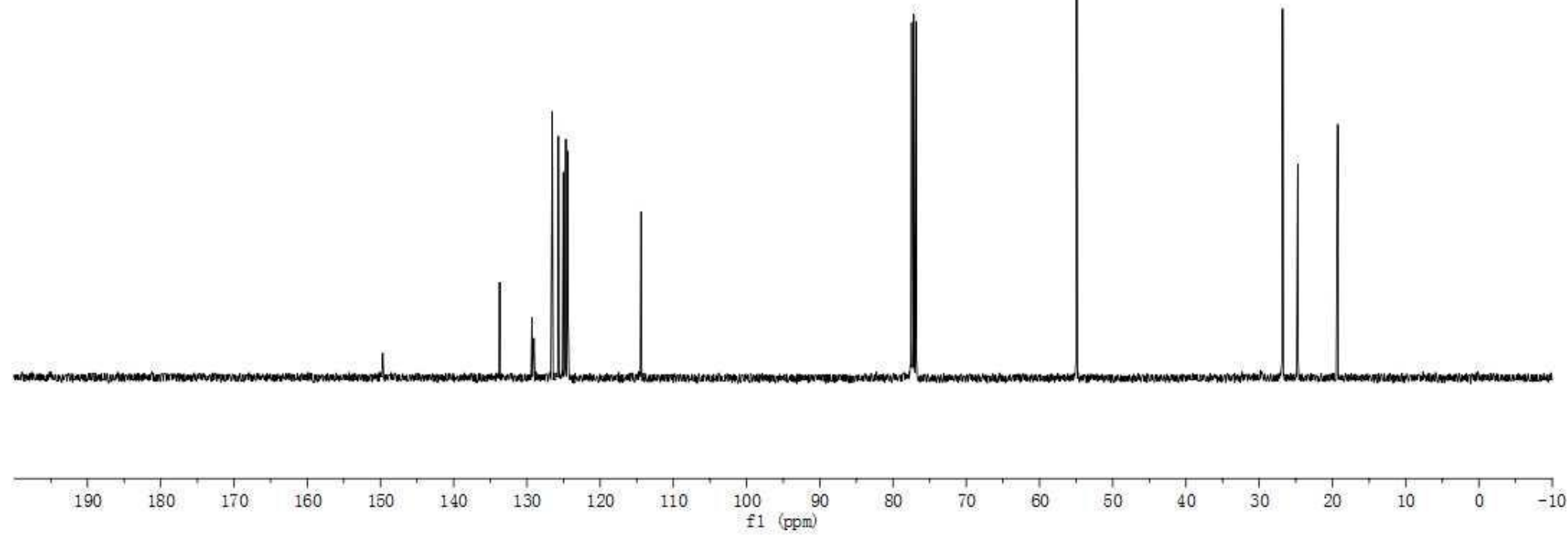

S131 


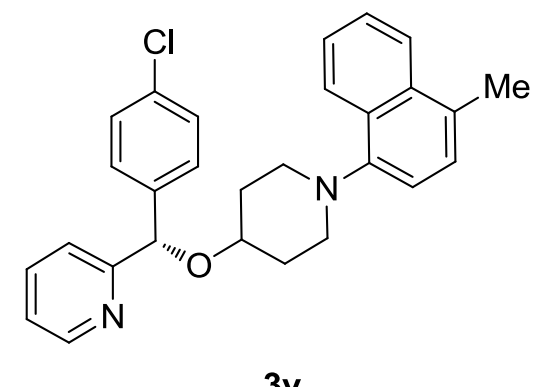

${ }^{1} \mathrm{H}$ NMR $\left(400 \mathrm{MHz}, \mathrm{CDCl}_{3}\right)$

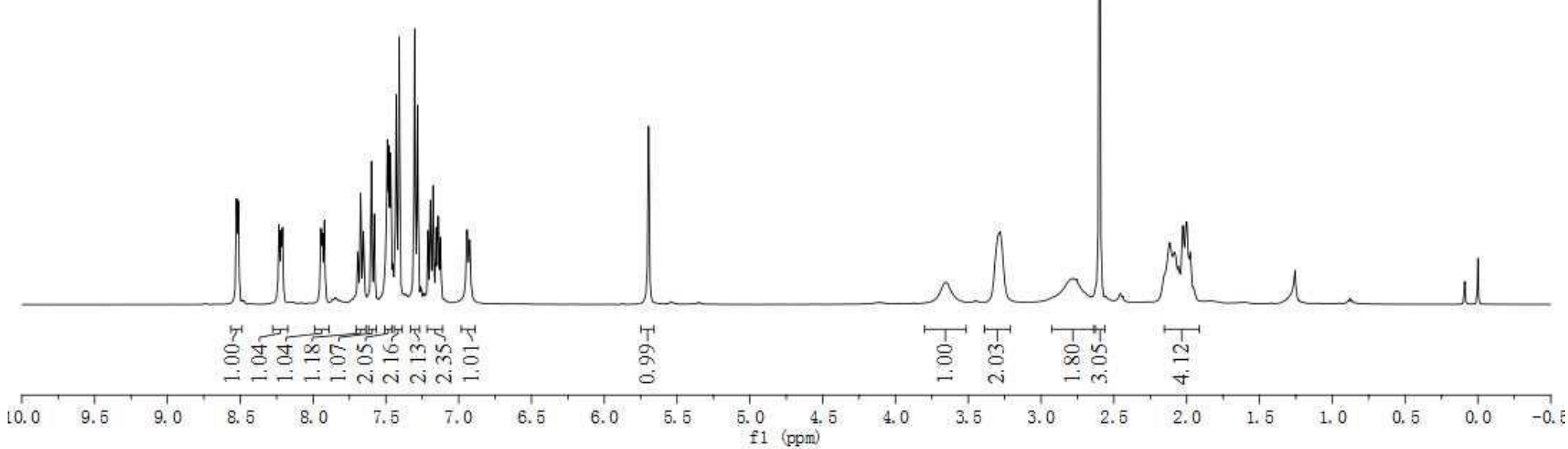



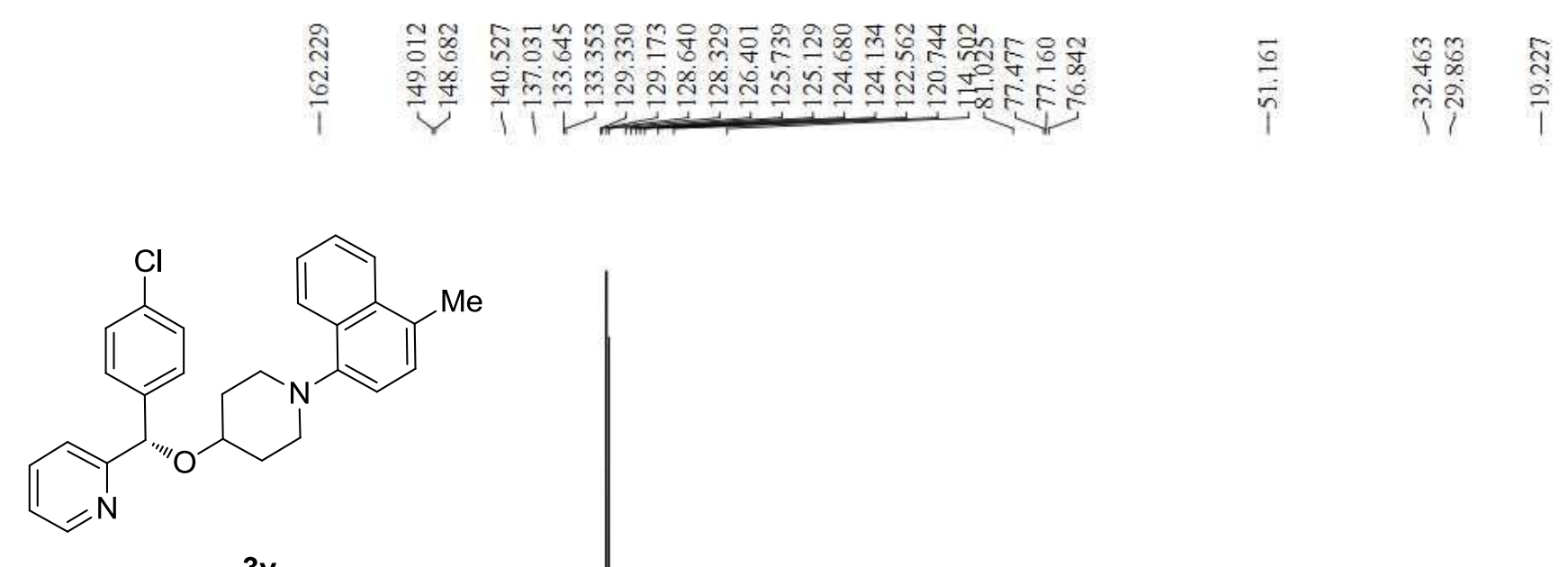

${ }^{13} \mathrm{C}$ NMR (100 MHz, $\mathrm{CDCl}_{3}$ )

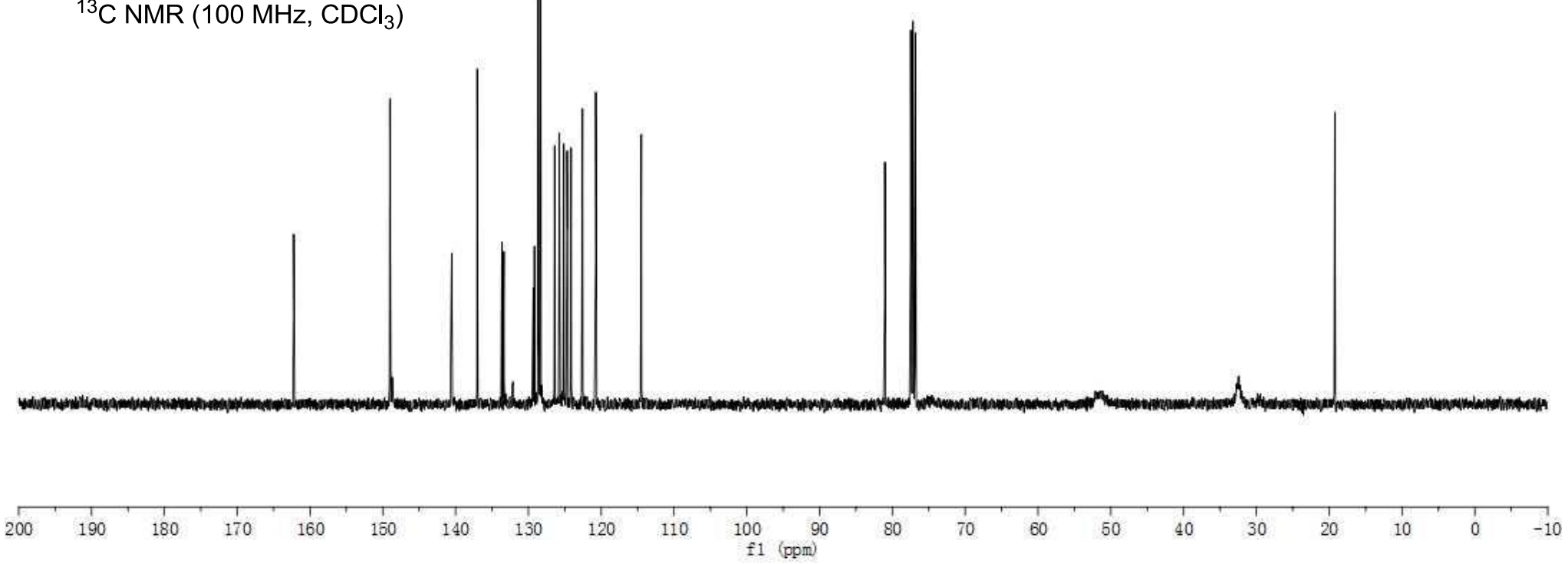




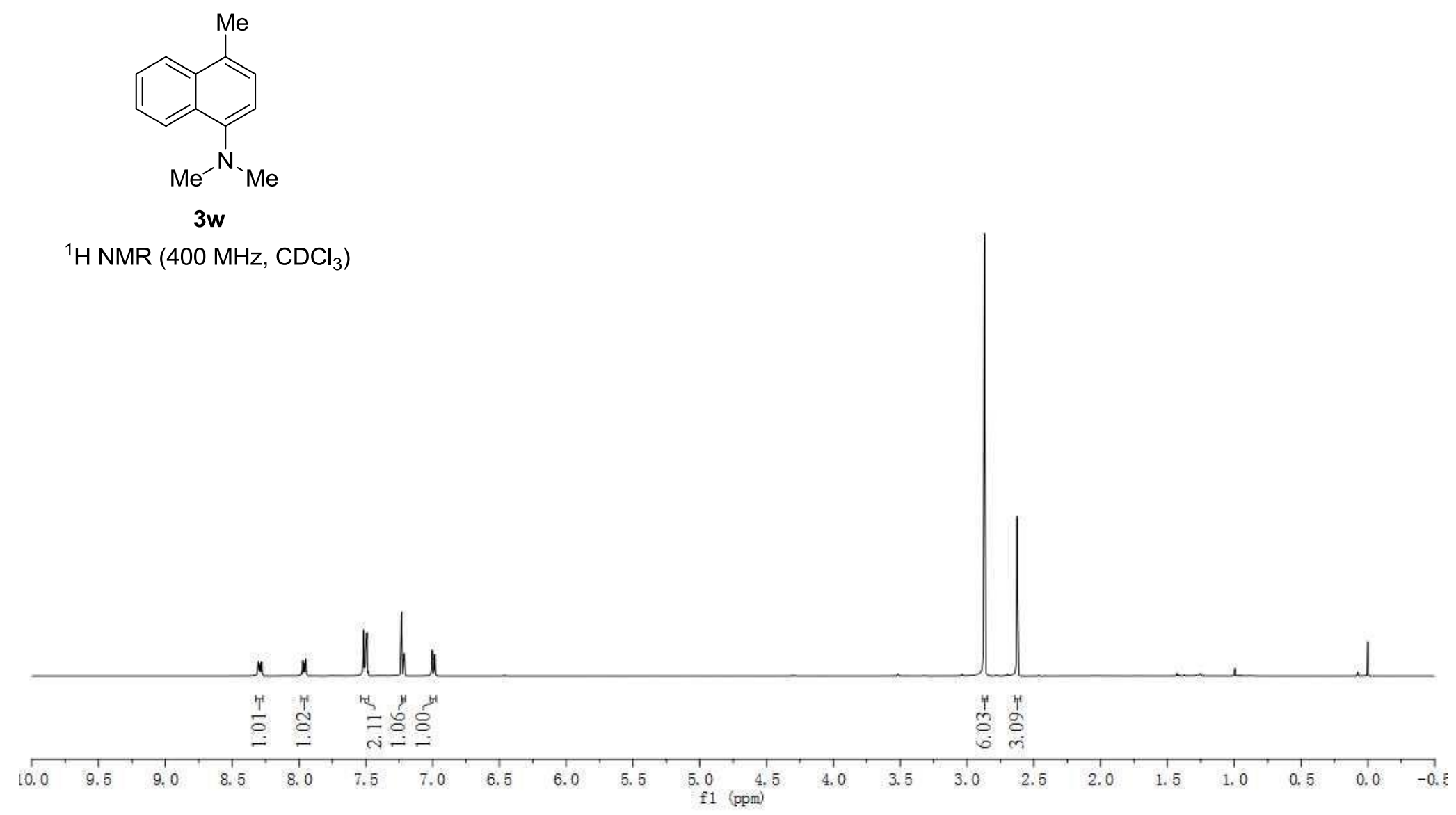



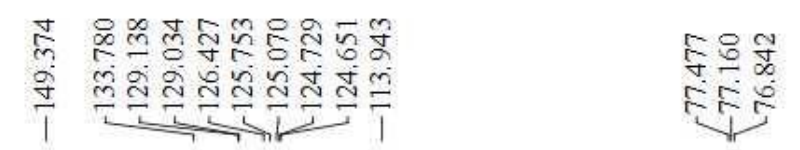

\begin{tabular}{ll}
$\infty$ \\
\multirow{1}{*}{$\frac{2}{1}$}
\end{tabular}

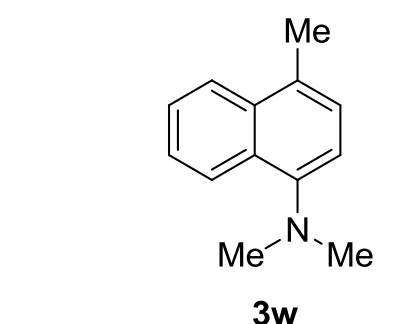

${ }^{13} \mathrm{C}$ NMR $\left(100 \mathrm{MHz}, \mathrm{CDCl}_{3}\right)$

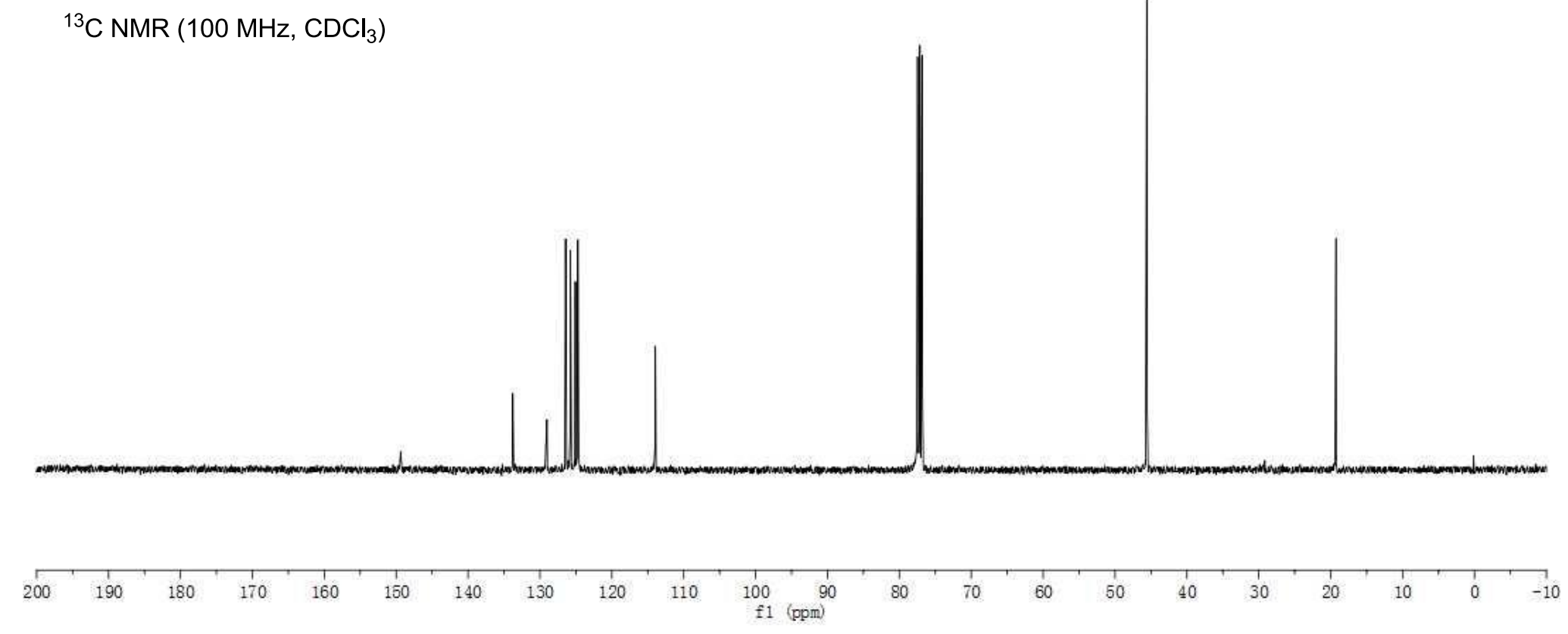

S135 


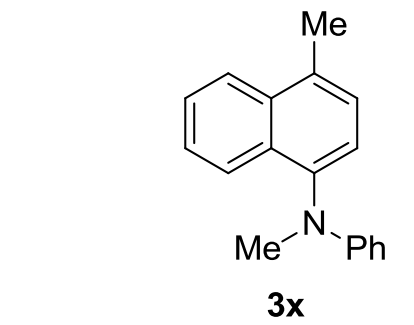

${ }^{1} \mathrm{H}$ NMR $\left(400 \mathrm{MHz}, \mathrm{CDCl}_{3}\right)$

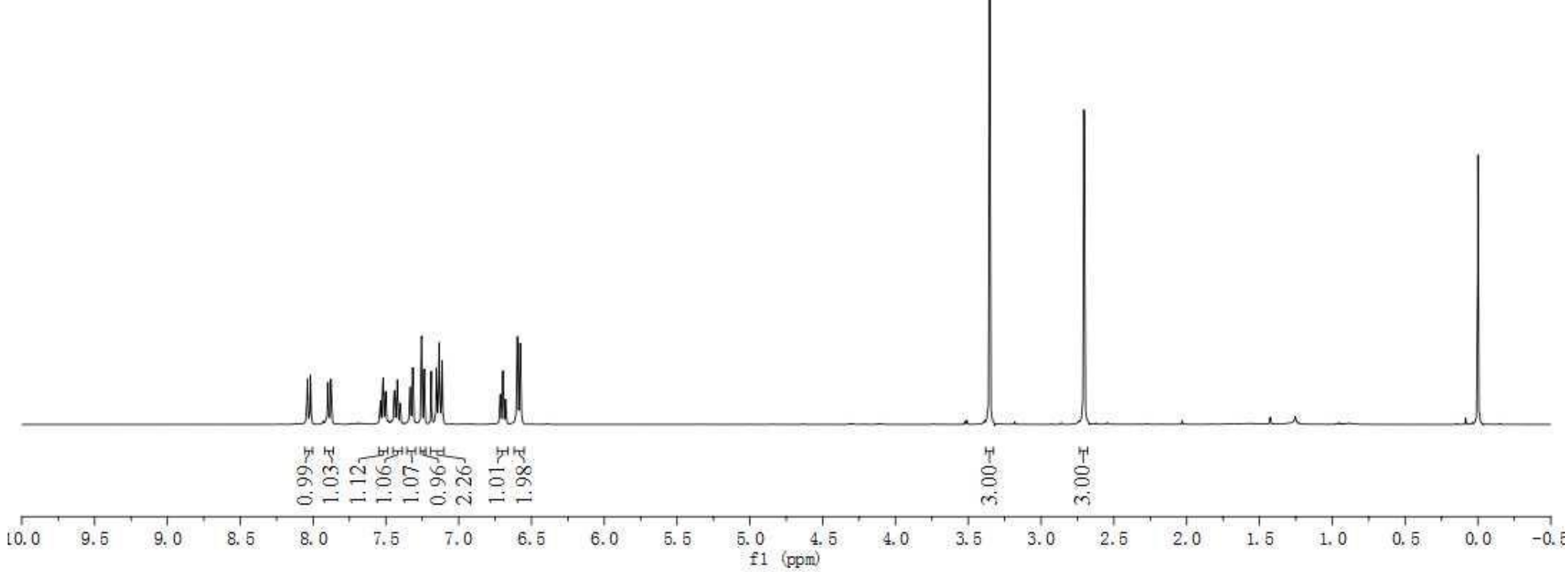



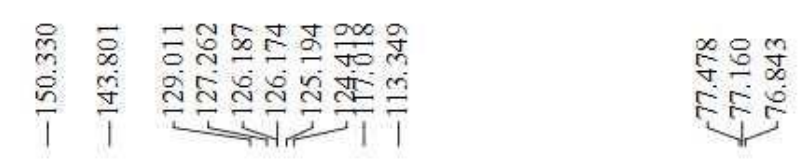

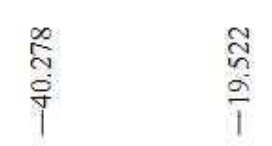
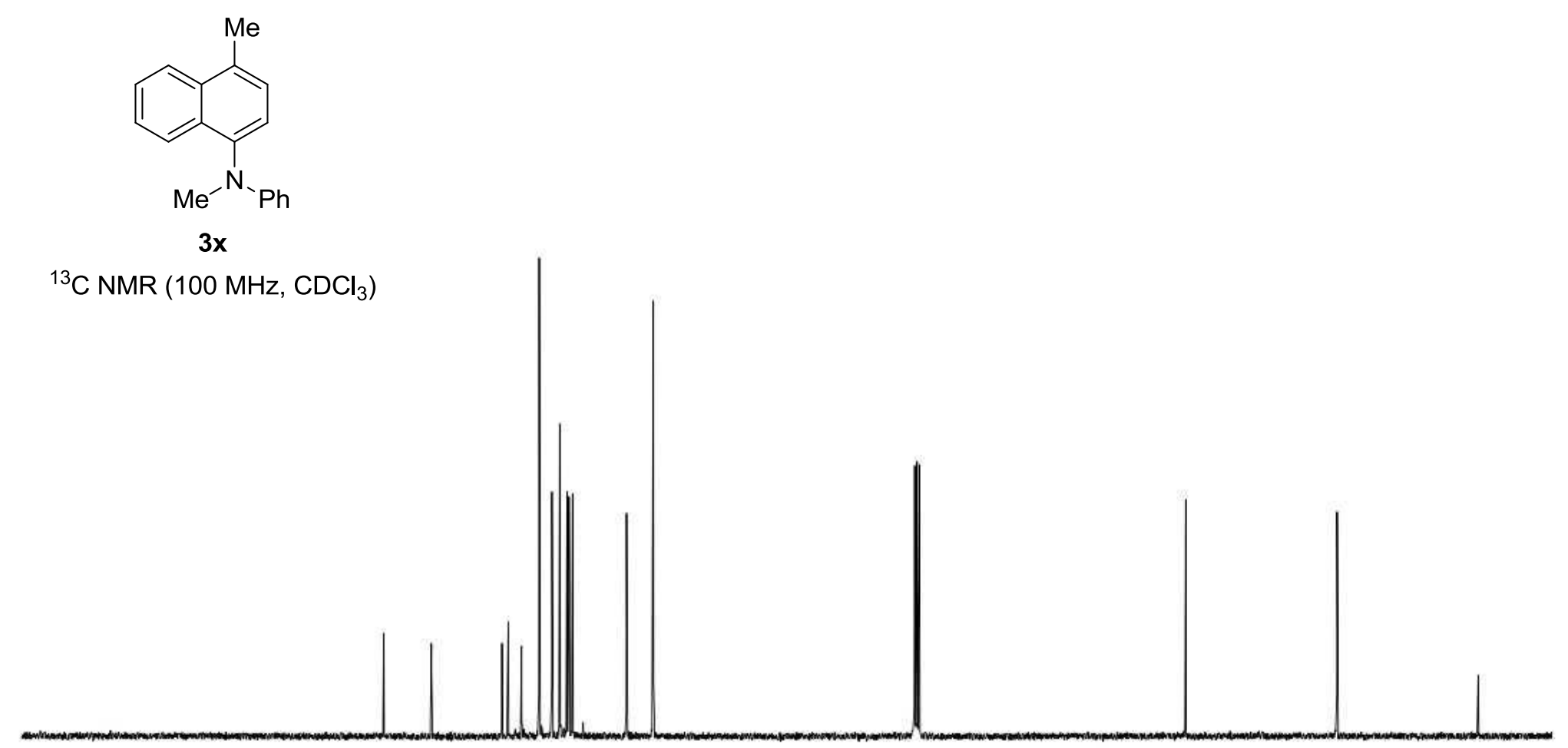

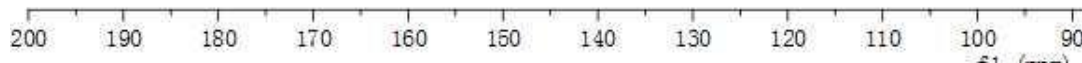




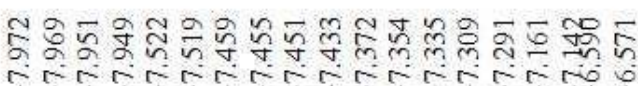

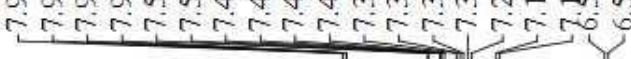

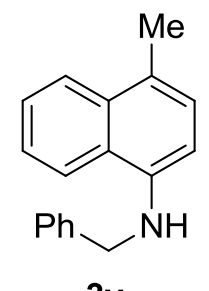

$3 y$

${ }^{1} \mathrm{H}$ NMR $\left(400 \mathrm{MHz}, \mathrm{CDCl}_{3}\right)$

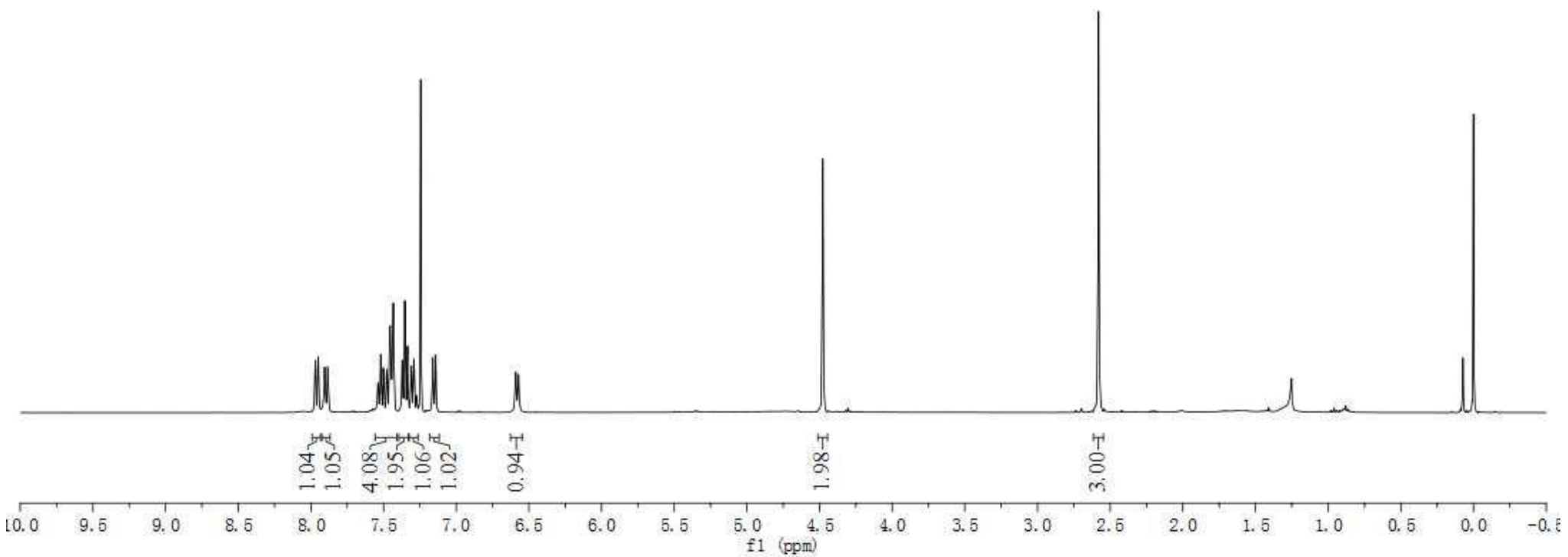

S138 

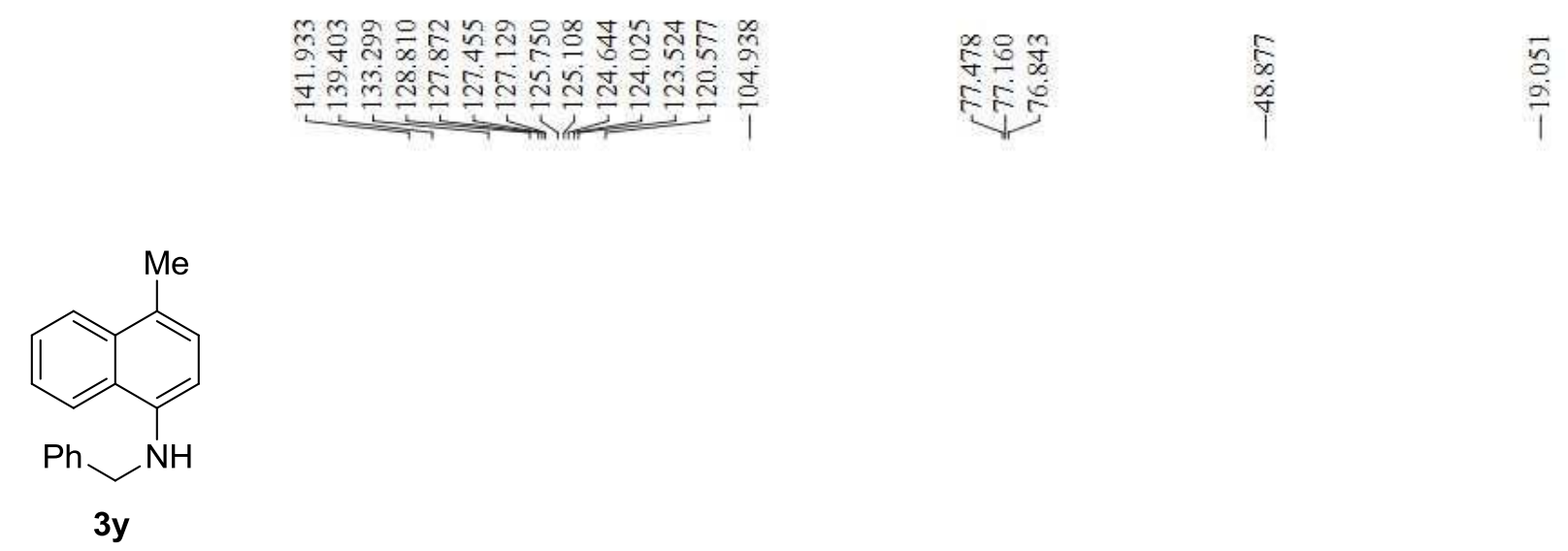

${ }^{13} \mathrm{C}$ NMR $\left(100 \mathrm{MHz}, \mathrm{CDCl}_{3}\right)$

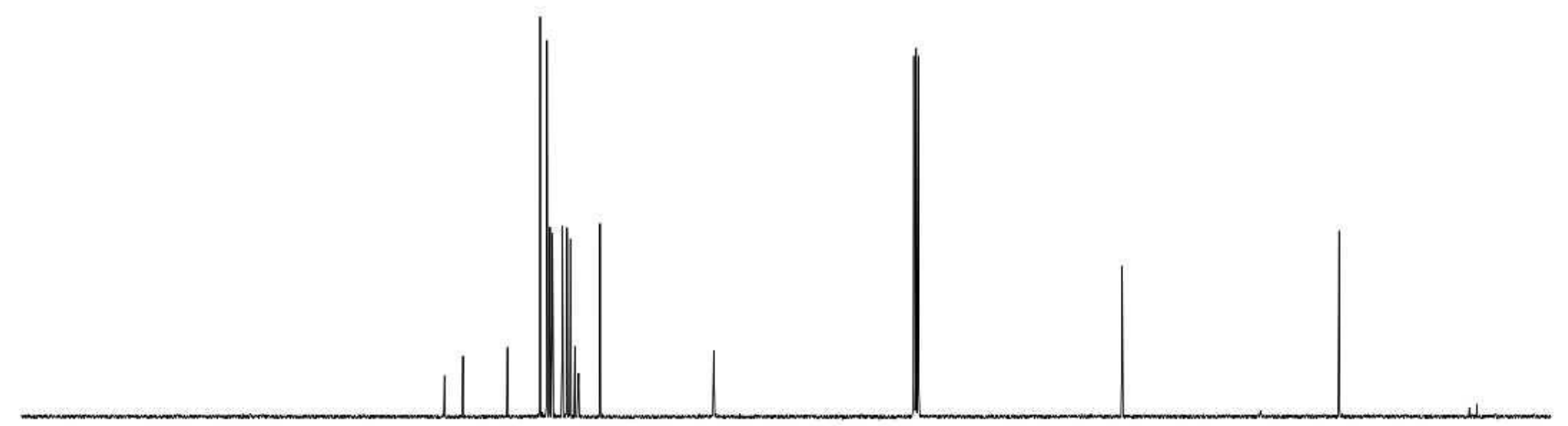

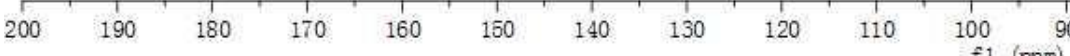




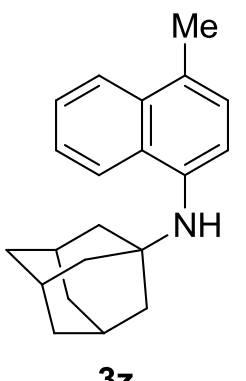

${ }^{1} \mathrm{H}$ NMR (400 MHz, $\mathrm{CDCl}_{3}$ )

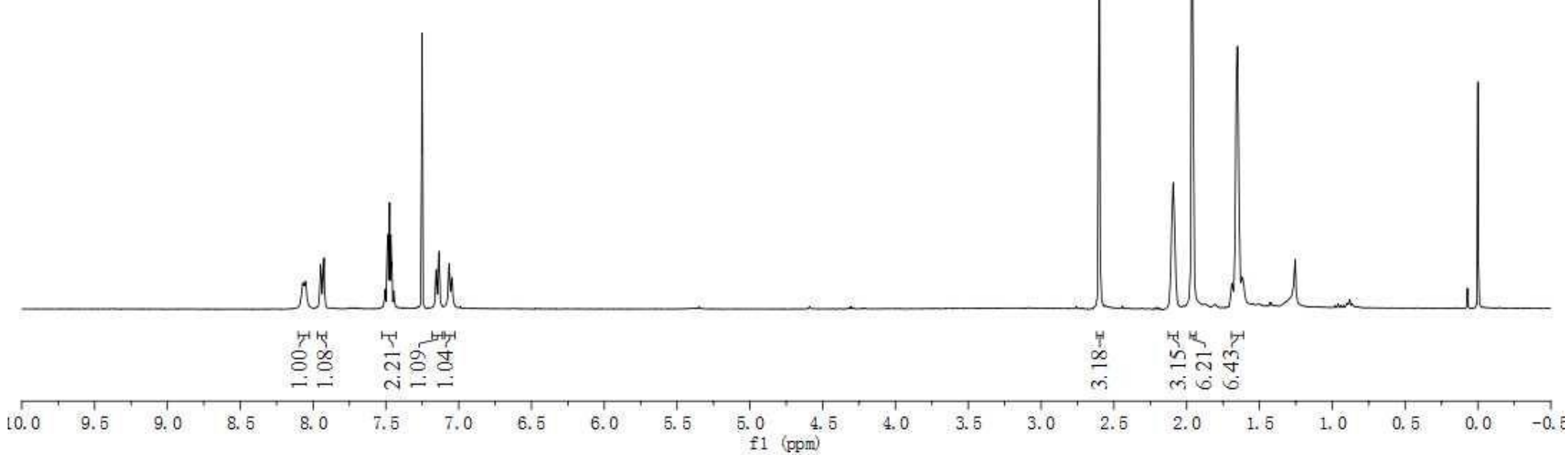



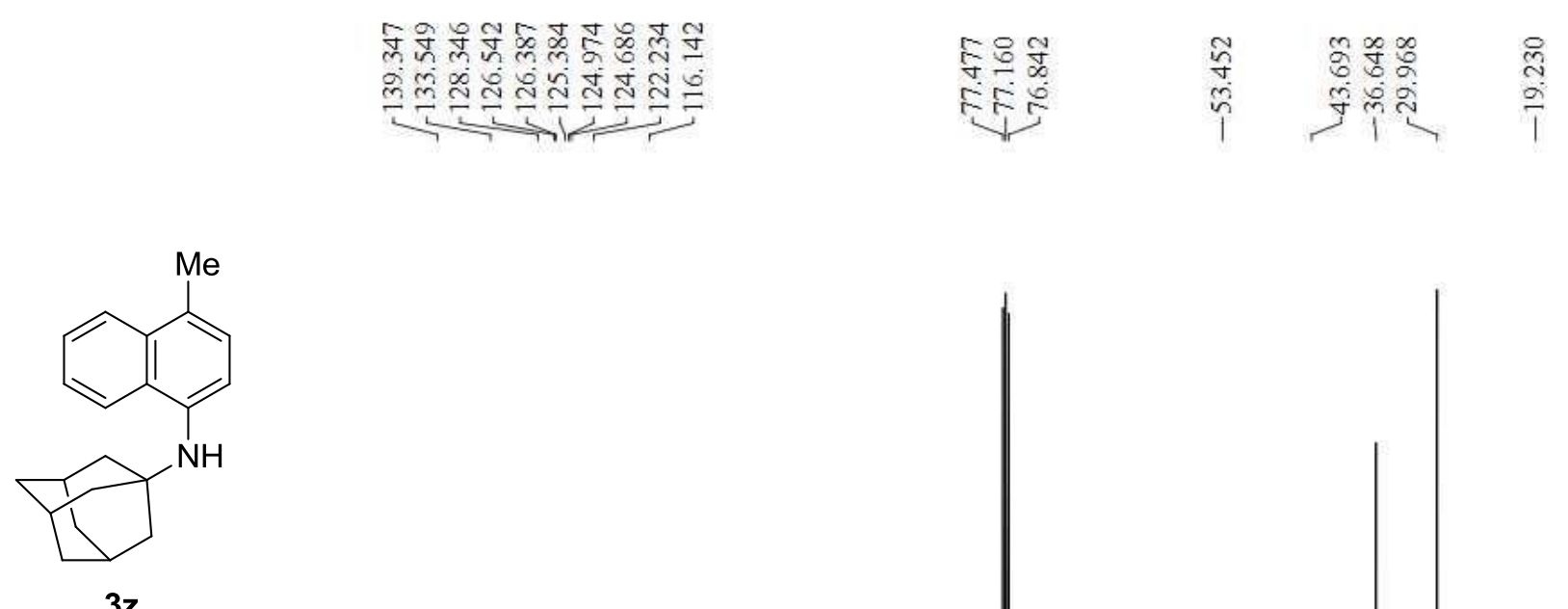

${ }^{13} \mathrm{C} \mathrm{NMR}\left(100 \mathrm{MHz}, \mathrm{CDCl}_{3}\right)$

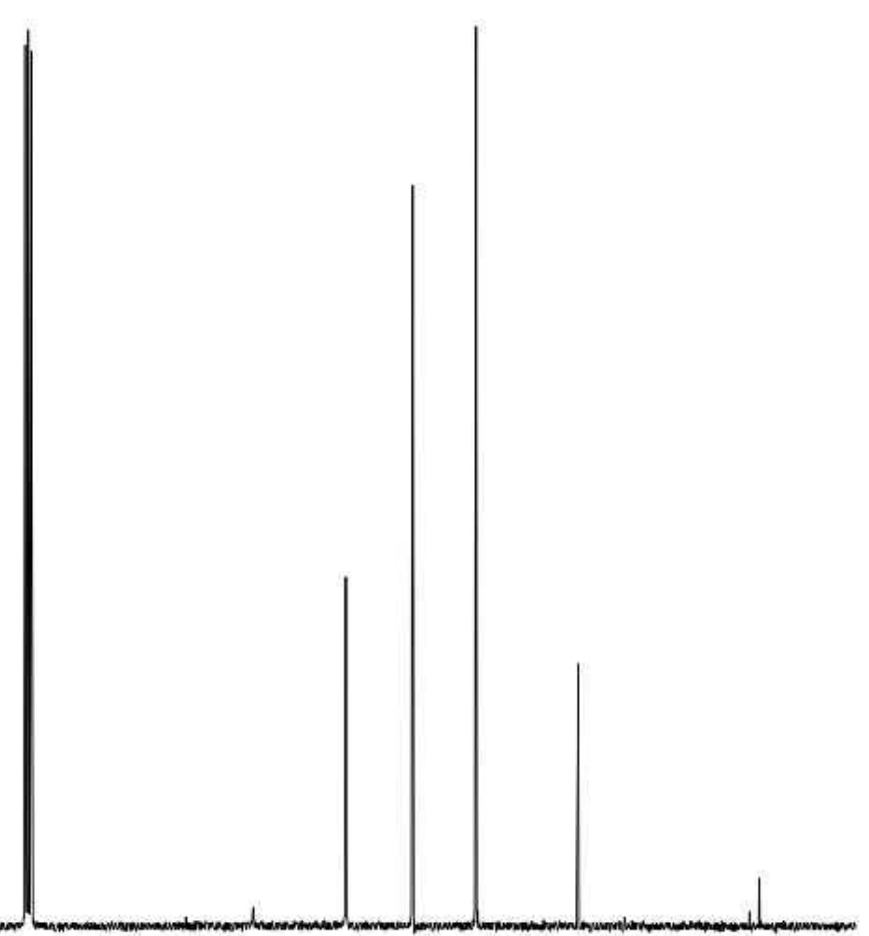

$\begin{array}{llllllllllll}200 & 190 & 180 & 170 & 160 & 150 & 140 & 130 & 120 & 110 & 100 & 90\end{array}$ 

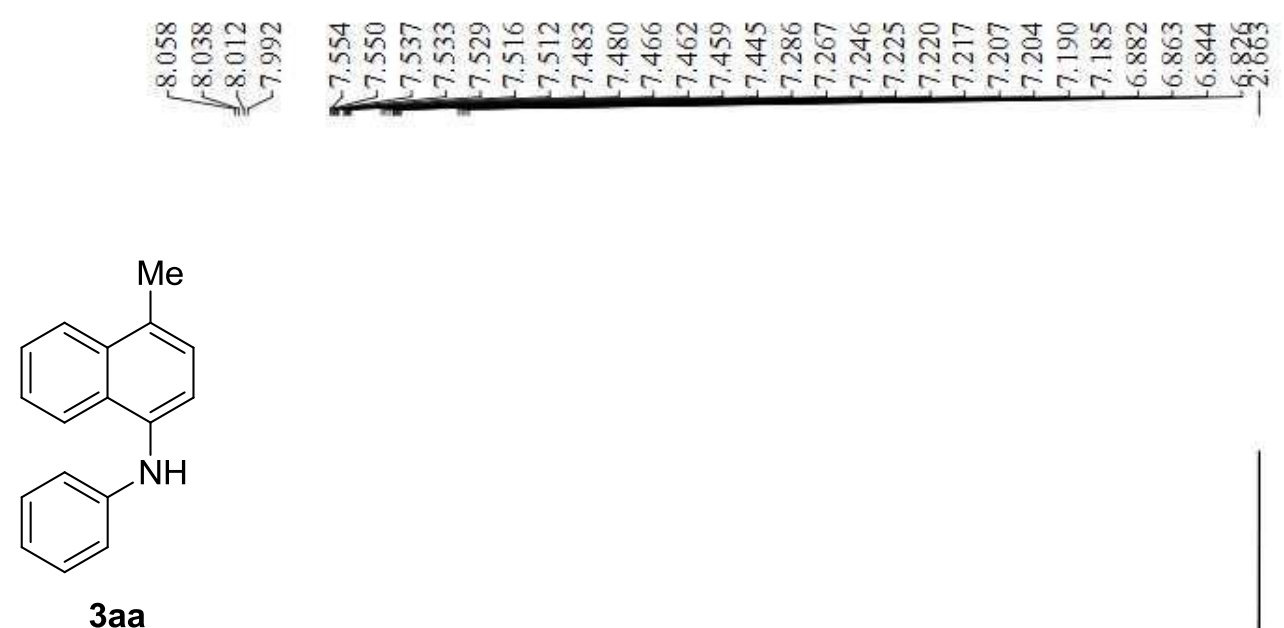

${ }^{1} \mathrm{H}$ NMR (400 MHz, $\mathrm{CDCl}_{3}$ )

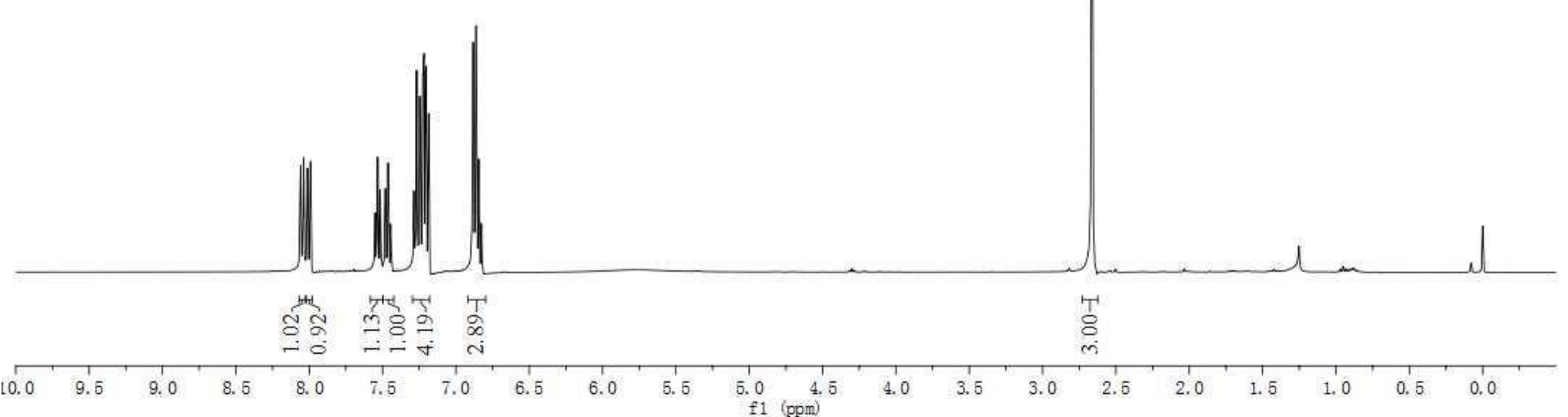

S142 


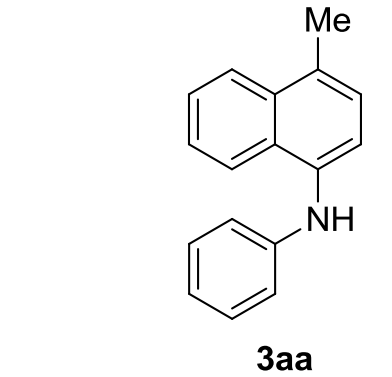

${ }^{13} \mathrm{C}$ NMR $\left(100 \mathrm{MHz}, \mathrm{CDCl}_{3}\right)$

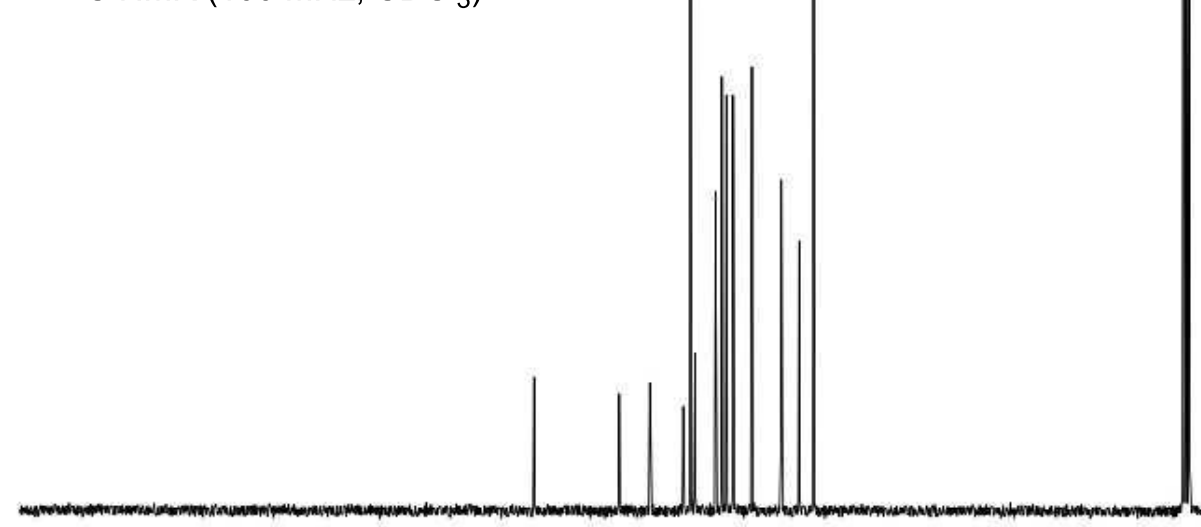




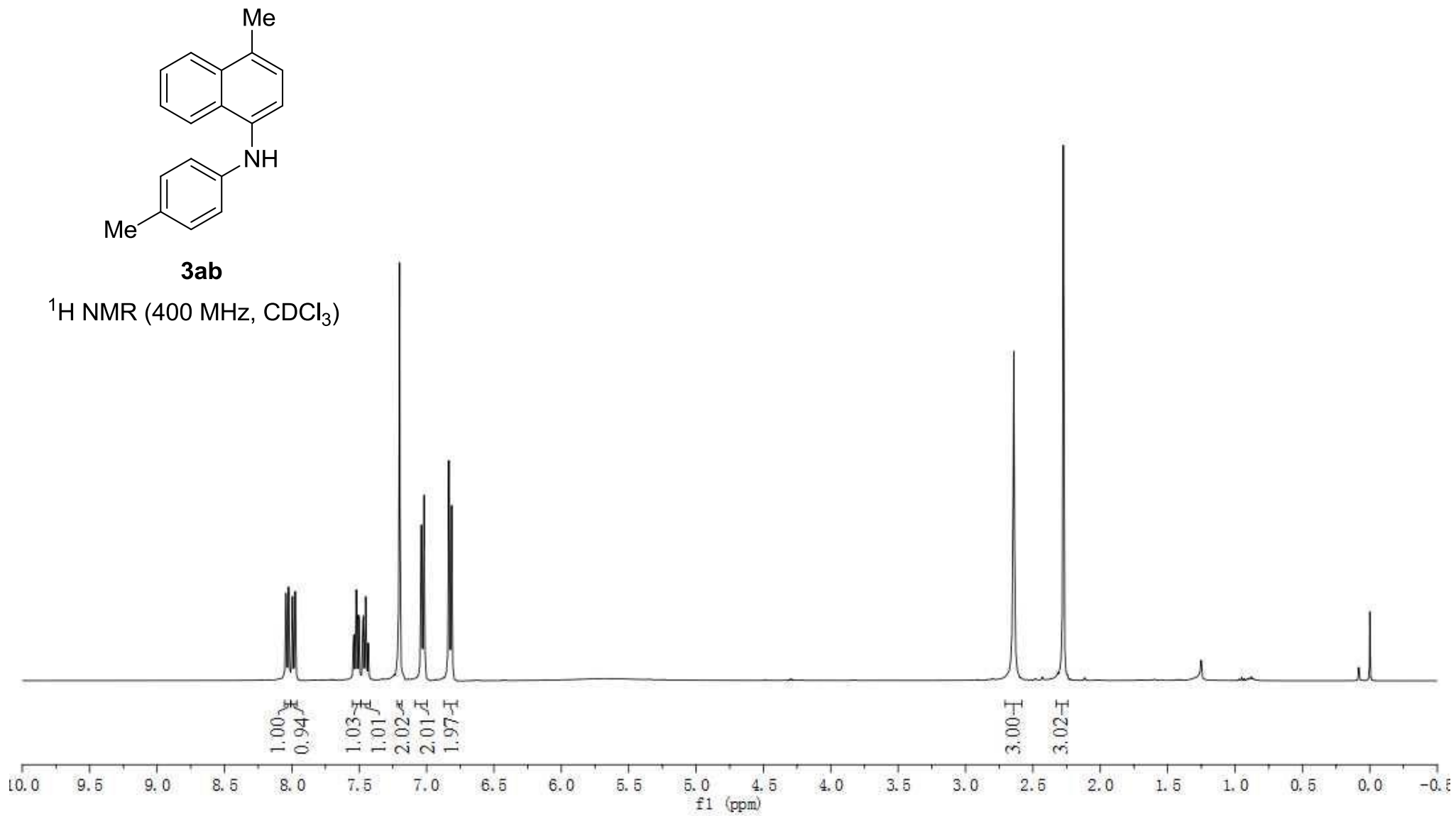

S144 


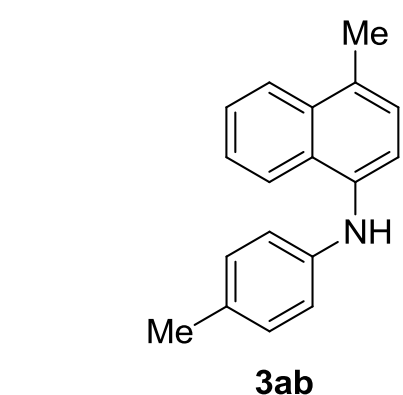

${ }^{13} \mathrm{C}$ NMR $\left(100 \mathrm{MHz}, \mathrm{CDCl}_{3}\right)$

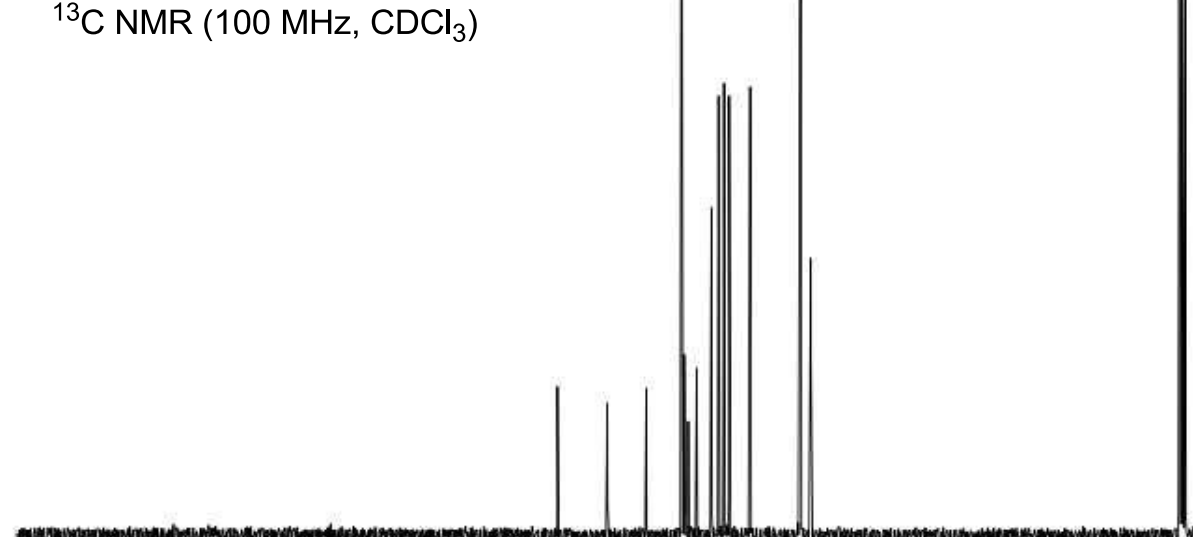

$$
200
$$
190
180 $170 \quad 160$ 150 140 $130 \quad 120$ 110 $\underset{\mathrm{fl}}{100 \mathrm{ppm})}$ 80 


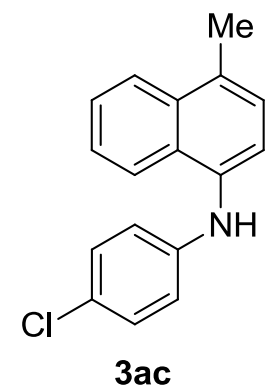

${ }^{1} \mathrm{H}$ NMR $\left(400 \mathrm{MHz}, \mathrm{CDCl}_{3}\right)$

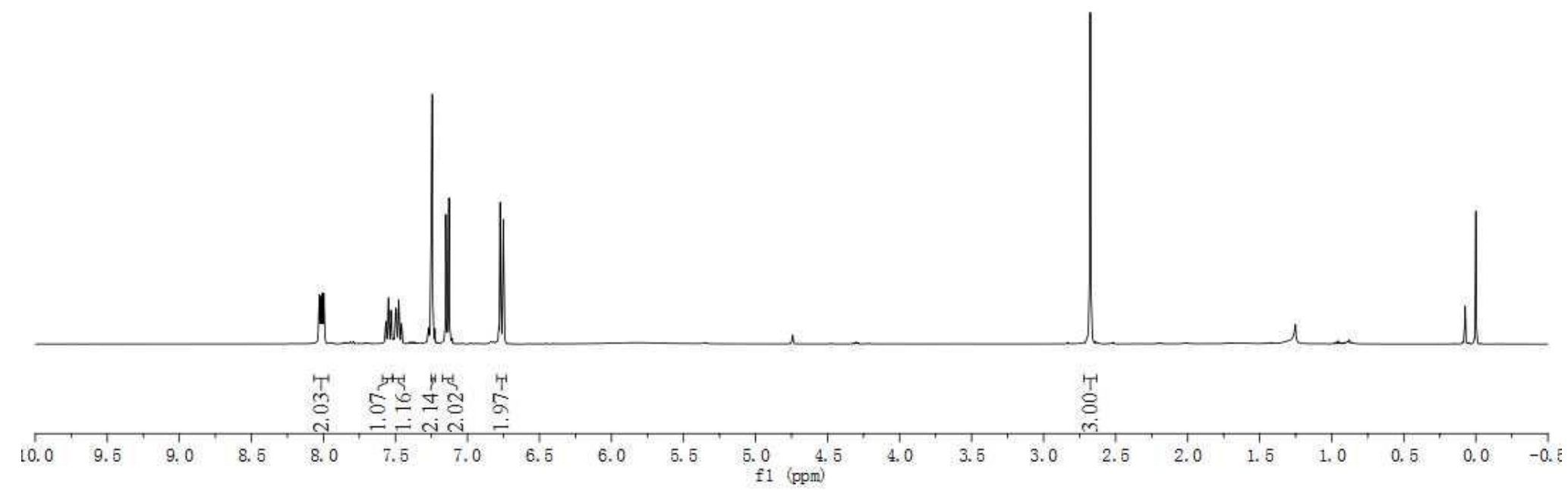

S146 


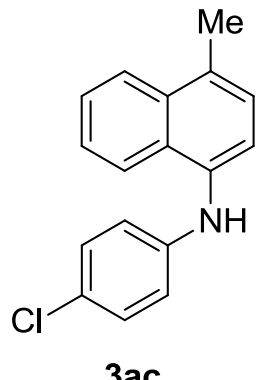

${ }^{13} \mathrm{C}$ NMR $\left(100 \mathrm{MHz}, \mathrm{CDCl}_{3}\right)$

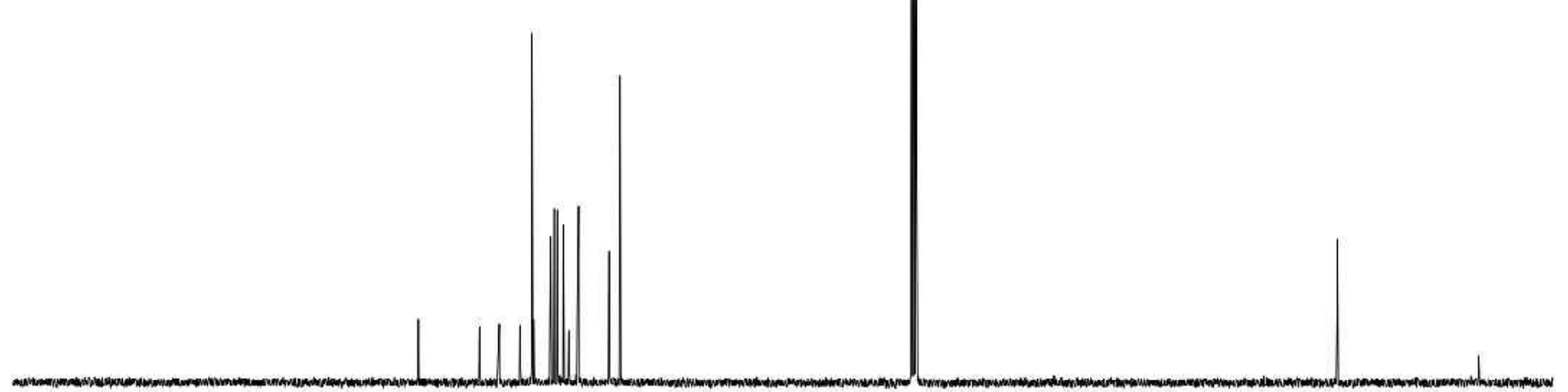

200 $190 \quad 180$

${ }_{170} \quad 160$

150

140

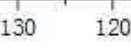

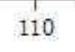

10090

80

1 60 


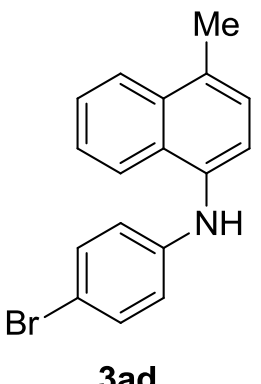

${ }^{1} \mathrm{H}$ NMR $\left(400 \mathrm{MHz}, \mathrm{CDCl}_{3}\right)$

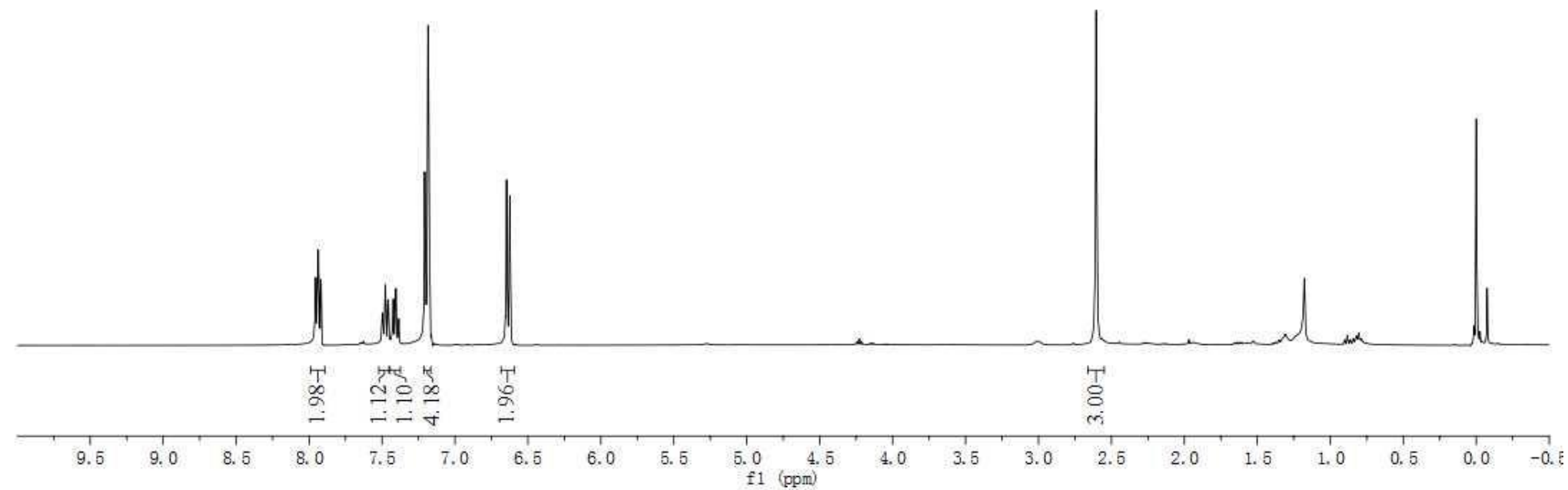



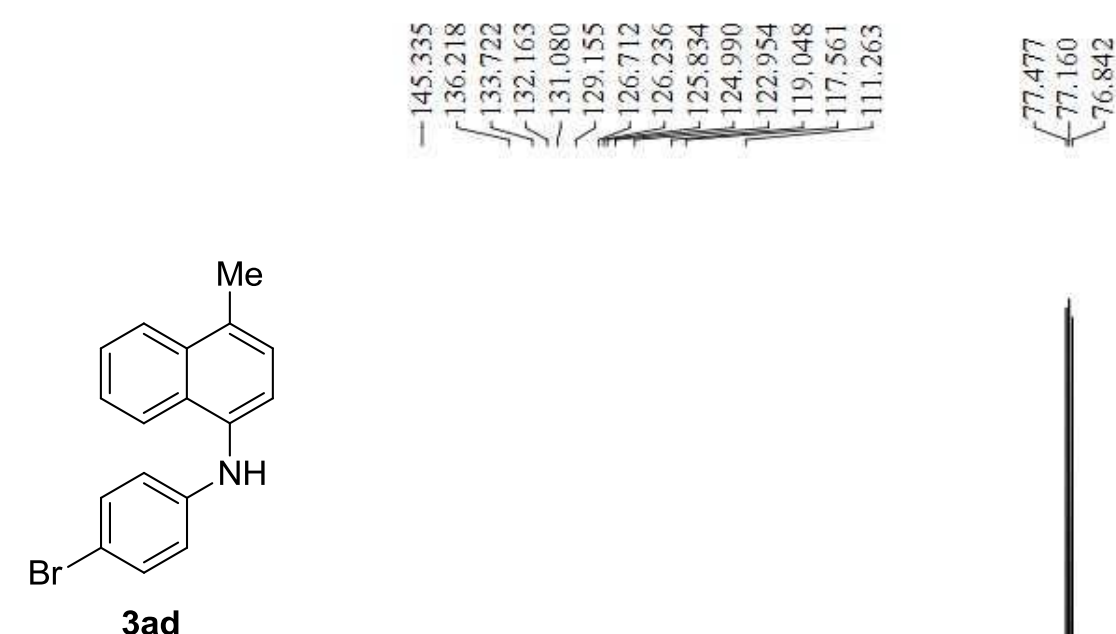

${ }^{13} \mathrm{C}$ NMR $\left(100 \mathrm{MHz}, \mathrm{CDCl}_{3}\right)$

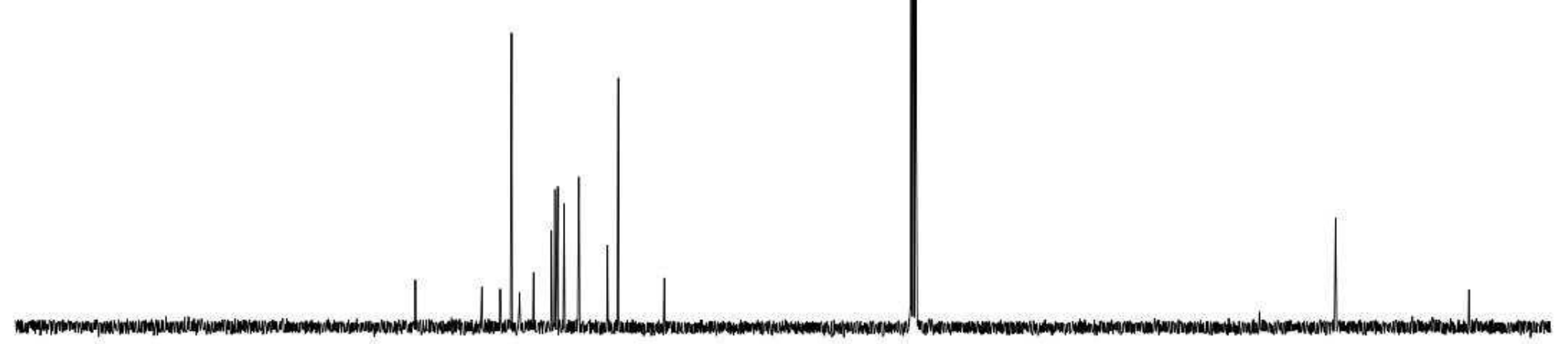

1 $1,1,1,1$

$150 \quad 140$

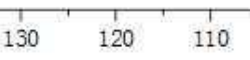

$100 \quad 90$

S149 


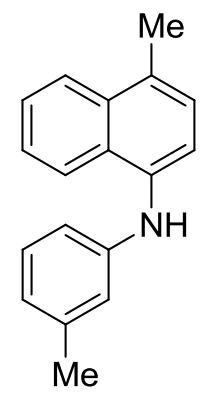

3ae

${ }^{1} \mathrm{H}$ NMR (400 MHz, $\left.\mathrm{CDCl}_{3}\right)$

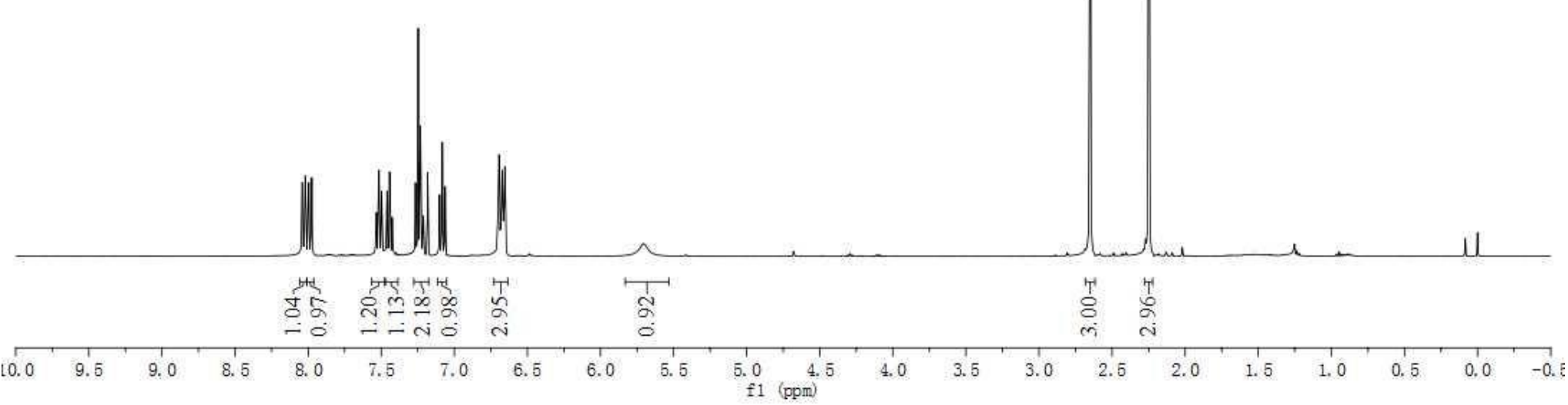




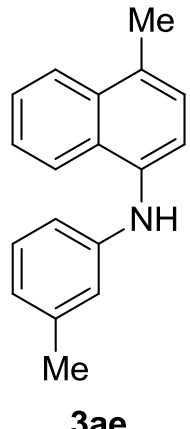

${ }^{13} \mathrm{C} \mathrm{NMR}\left(100 \mathrm{MHz}, \mathrm{CDCl}_{3}\right)$

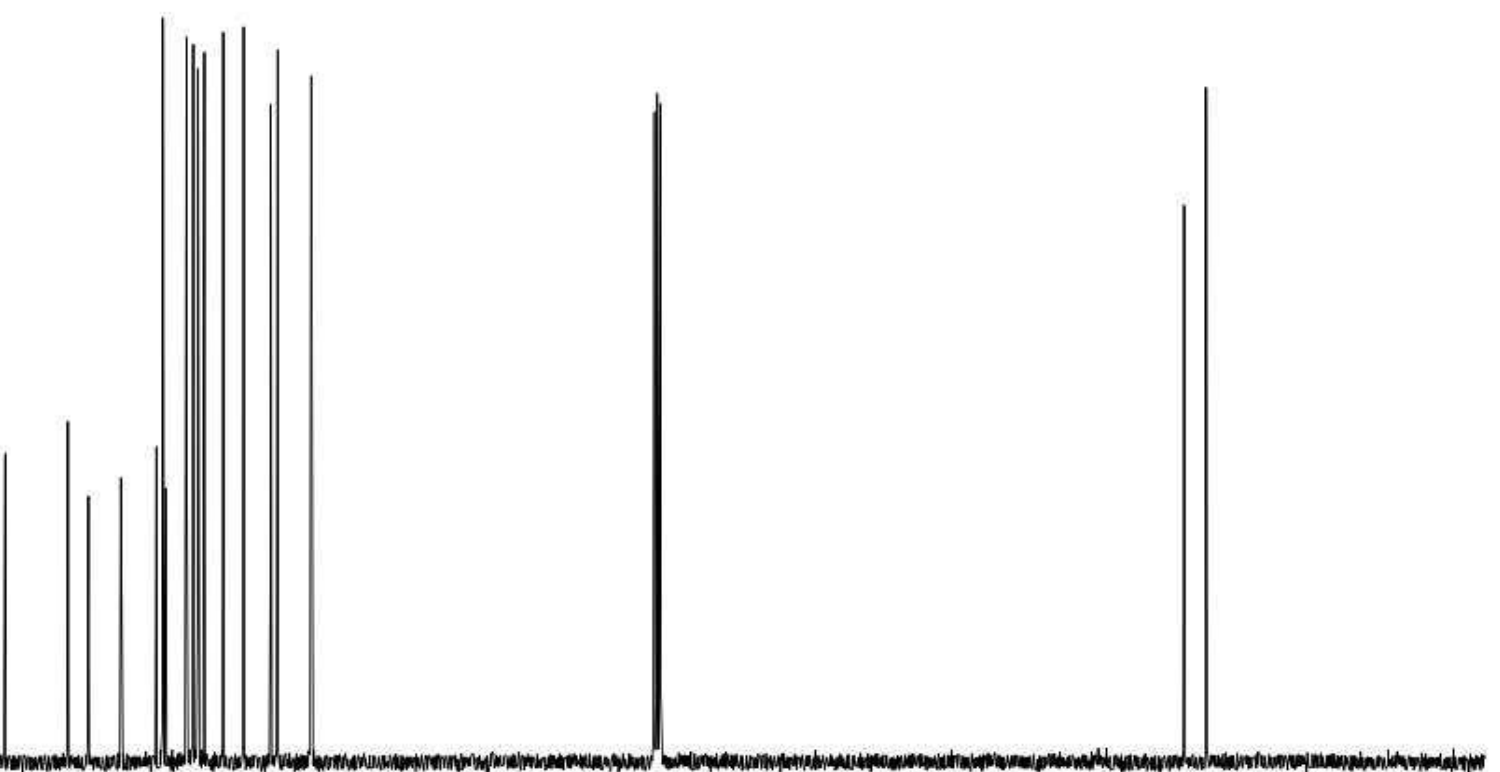

200 $170 \quad 160$ 160 $140 \quad 130$ $120 \quad 110$ 10090 80 
旁解

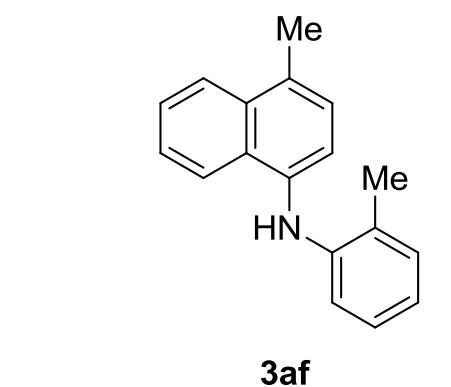

${ }^{1} \mathrm{H} \mathrm{NMR}\left(400 \mathrm{MHz}, \mathrm{CDCl}_{3}\right)$

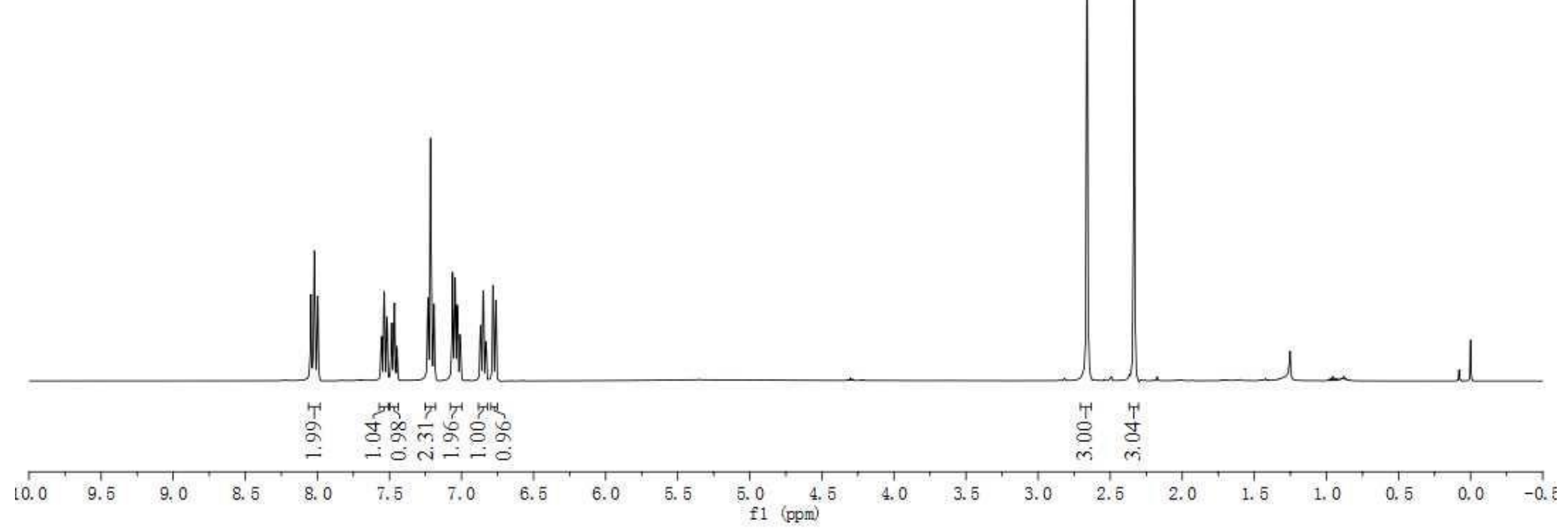

S152 


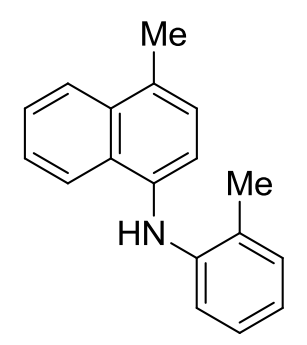

3af

${ }^{13} \mathrm{C}$ NMR $\left(100 \mathrm{MHz}, \mathrm{CDCl}_{3}\right.$ )

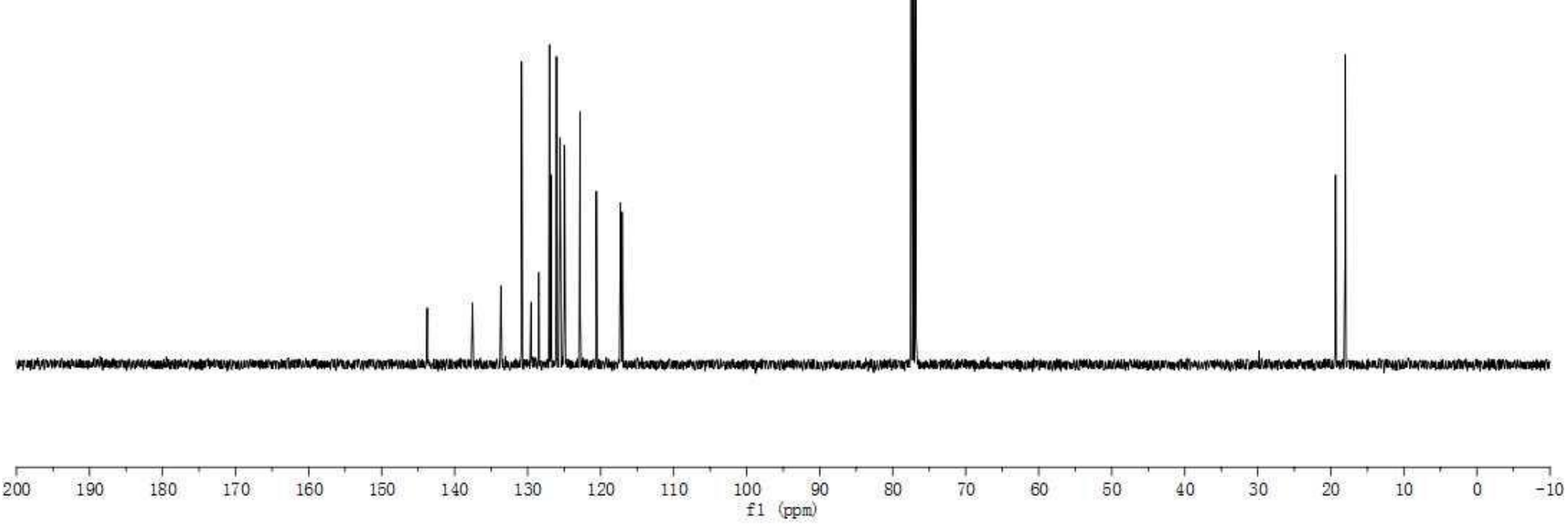

S153 


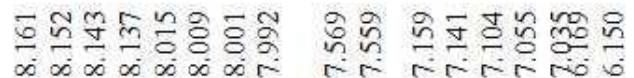

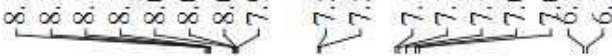

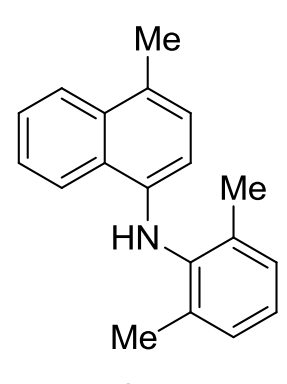

${ }^{1} \mathrm{H}$ NMR $\left(400 \mathrm{MHz}, \mathrm{CDCl}_{3}\right)$

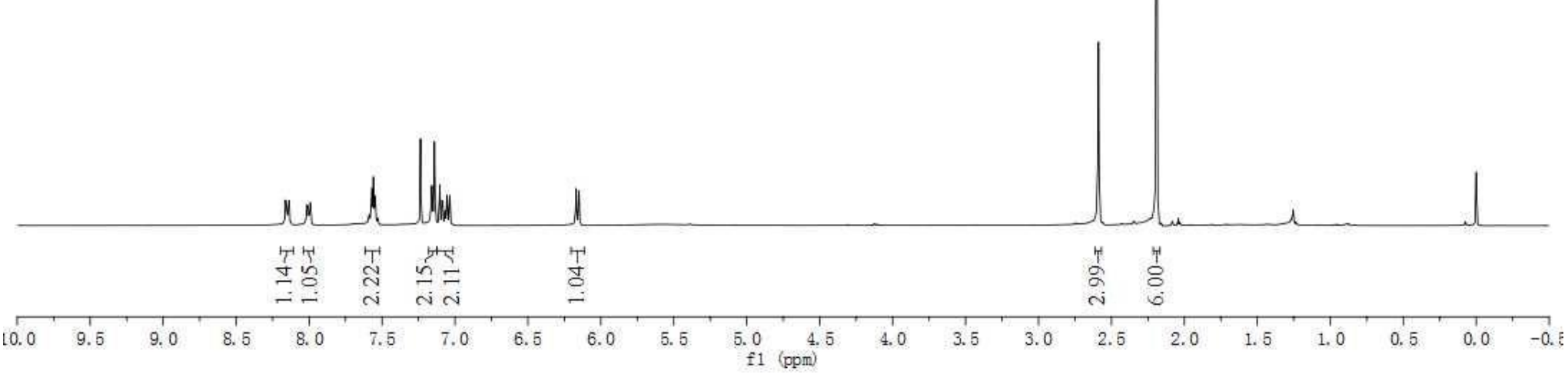

S154 $\overbrace{i}^{\infty} \frac{5}{1}$

\begin{tabular}{l}
8 \\
8 \\
\hline \\
$i$
\end{tabular} 

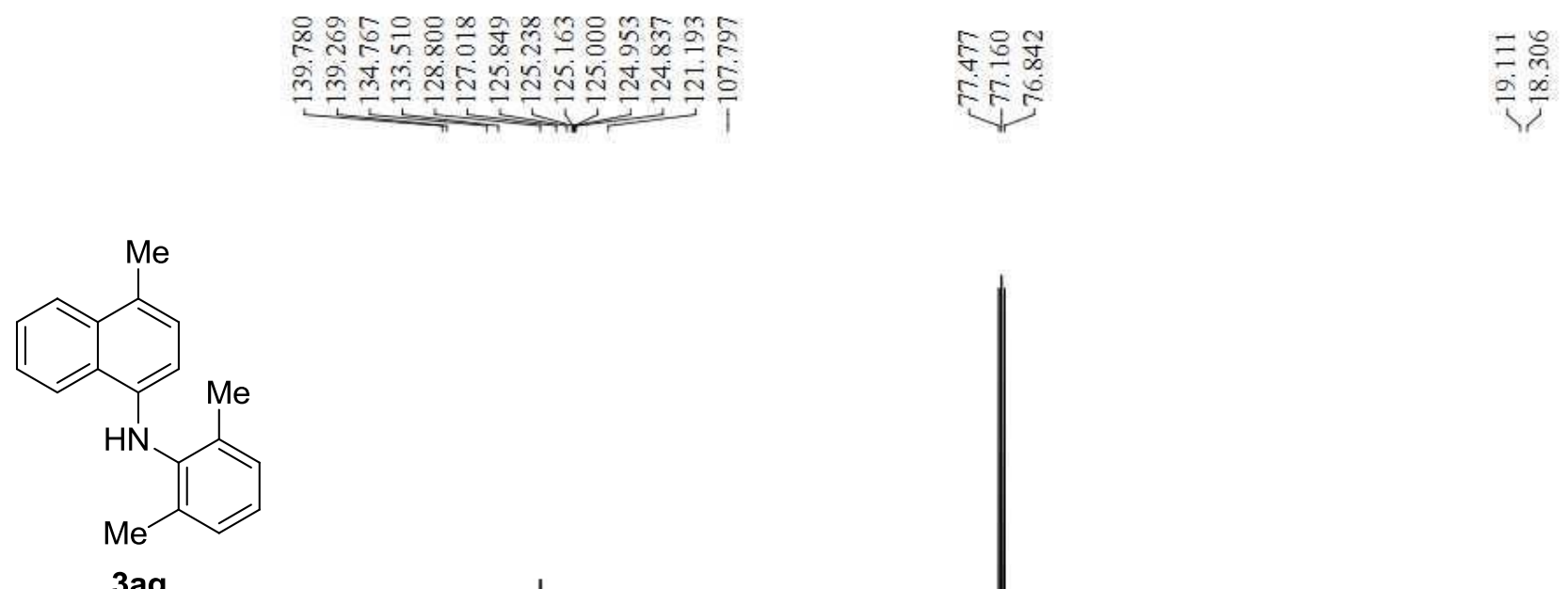

${ }^{13} \mathrm{C} \mathrm{NMR}\left(100 \mathrm{MHz}, \mathrm{CDCl}_{3}\right)$

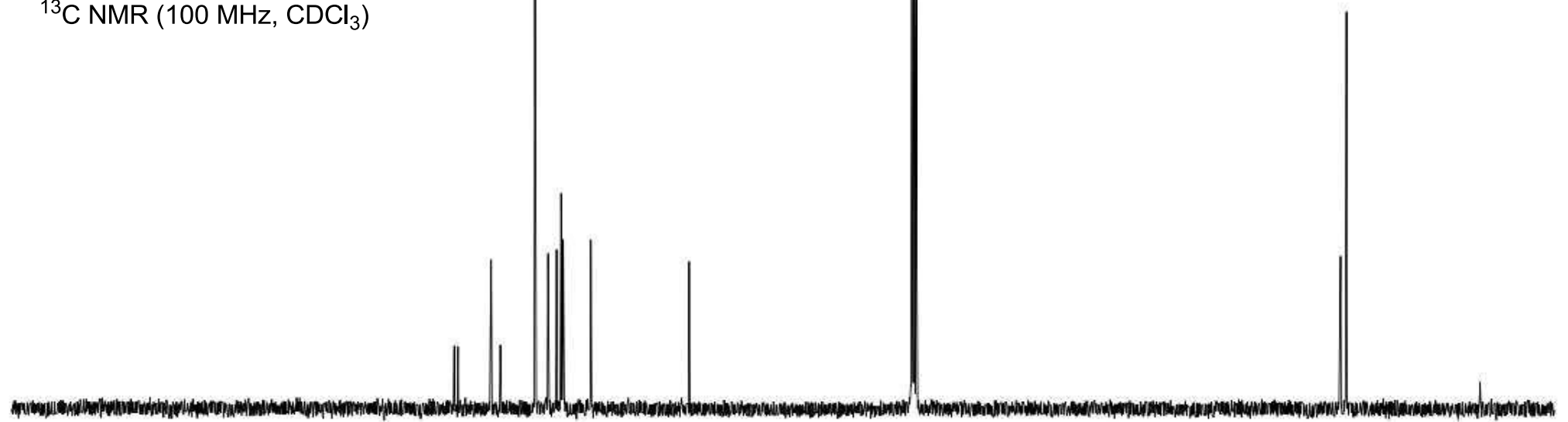

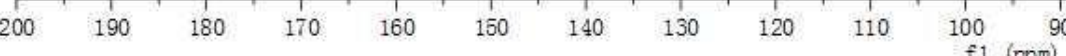




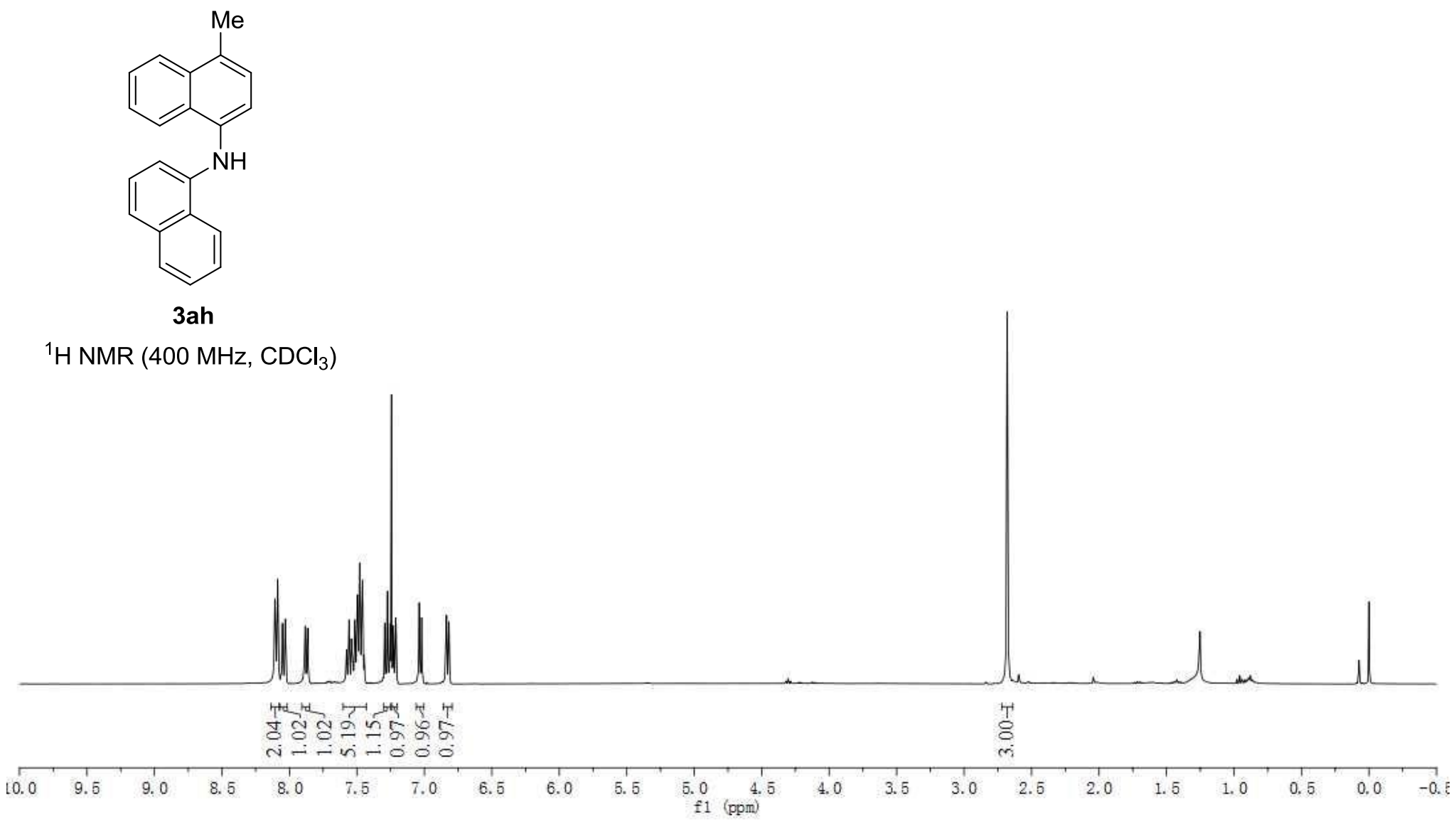




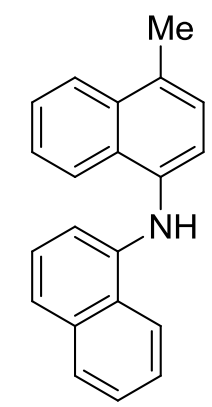

3ah

${ }^{13} \mathrm{C} \mathrm{NMR}\left(100 \mathrm{MHz}, \mathrm{CDCl}_{3}\right)$

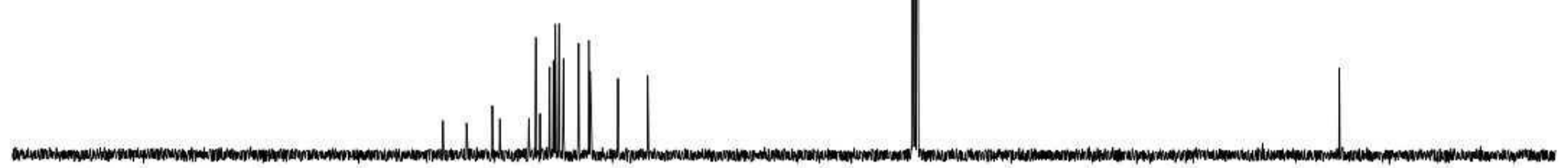

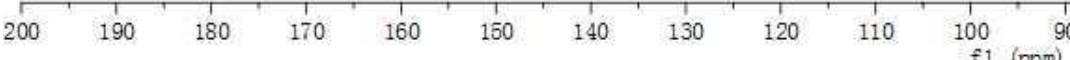




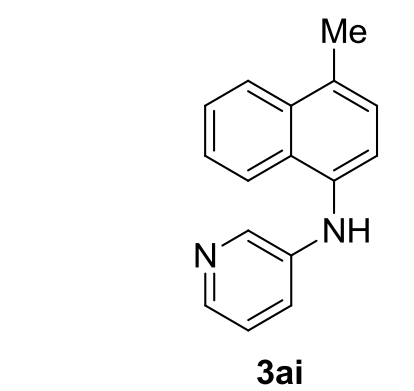

\section{${ }^{1} \mathrm{H}$ NMR $\left(400 \mathrm{MHz}, \mathrm{CDCl}_{3}\right)$}

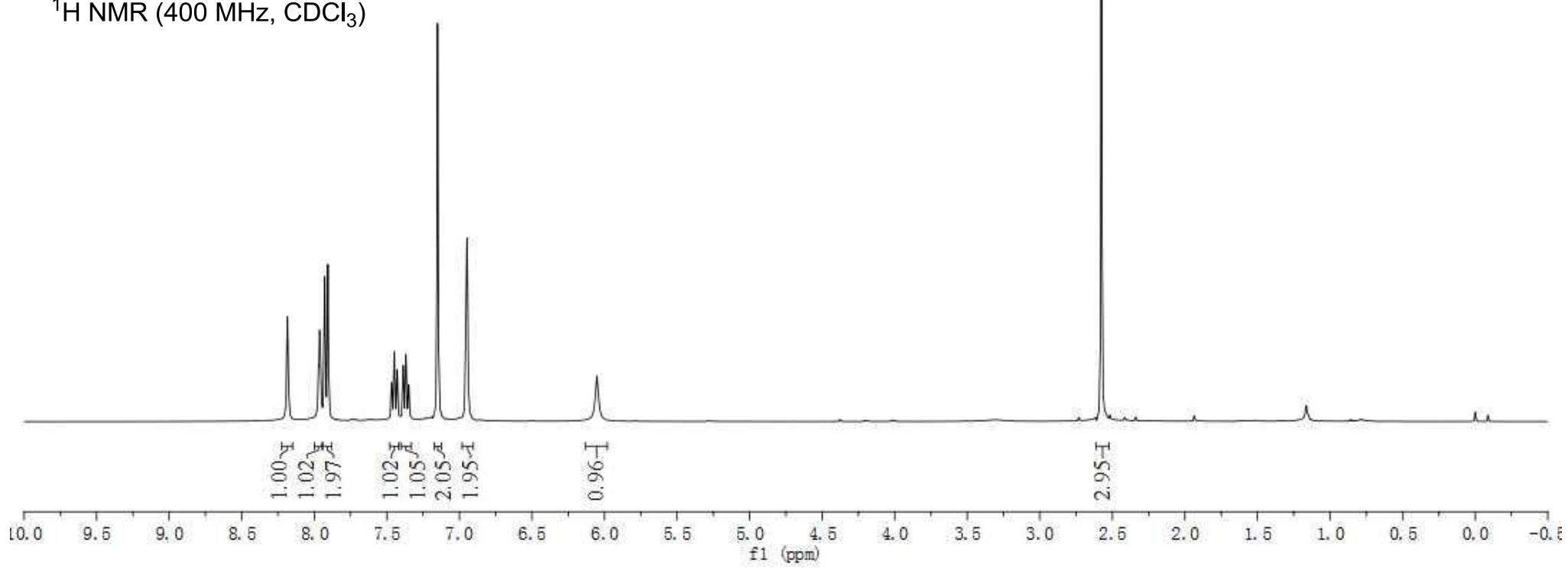




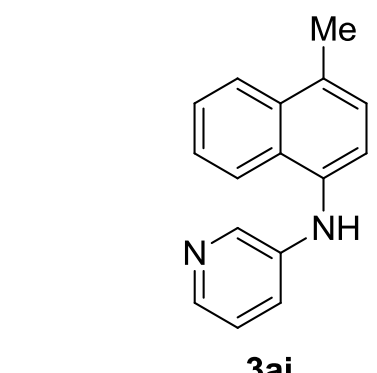

3ai

${ }^{13} \mathrm{C} \mathrm{NMR}\left(100 \mathrm{MHz}, \mathrm{CDCl}_{3}\right)$
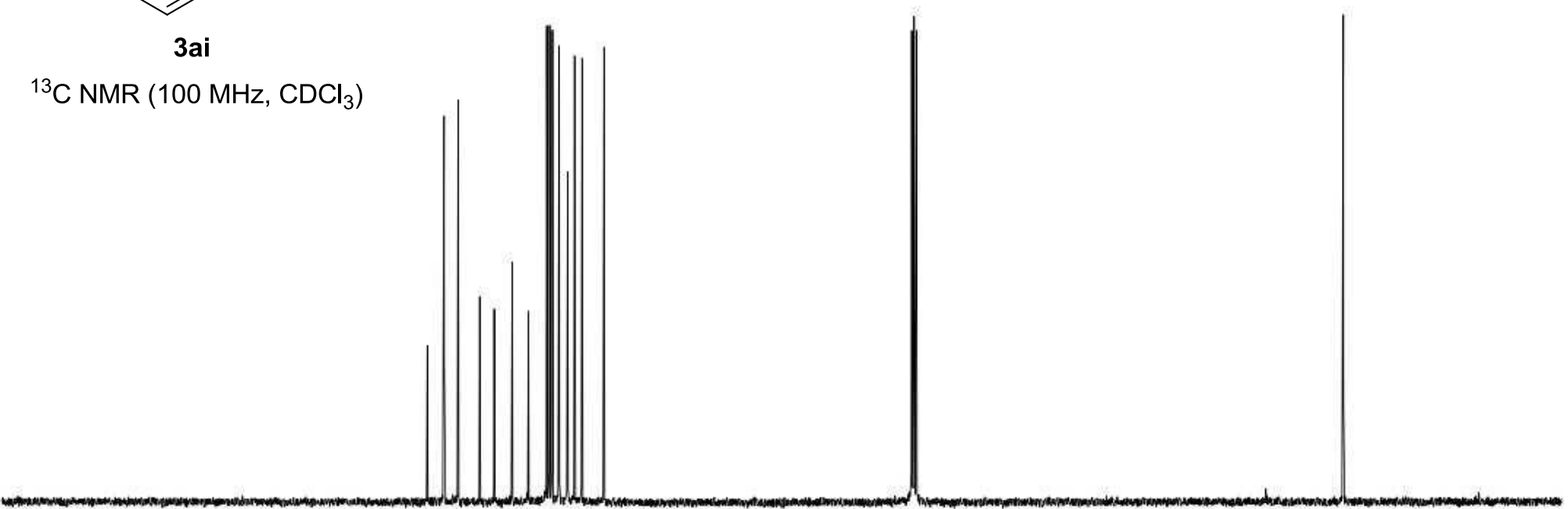

$200 \quad 190 \quad 180$

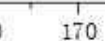

160

$150 \quad 140$

$130 \quad 120$

100,90

80

$1+\frac{1}{1}$

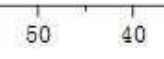

30

S159 


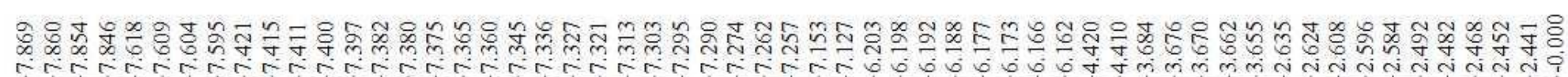

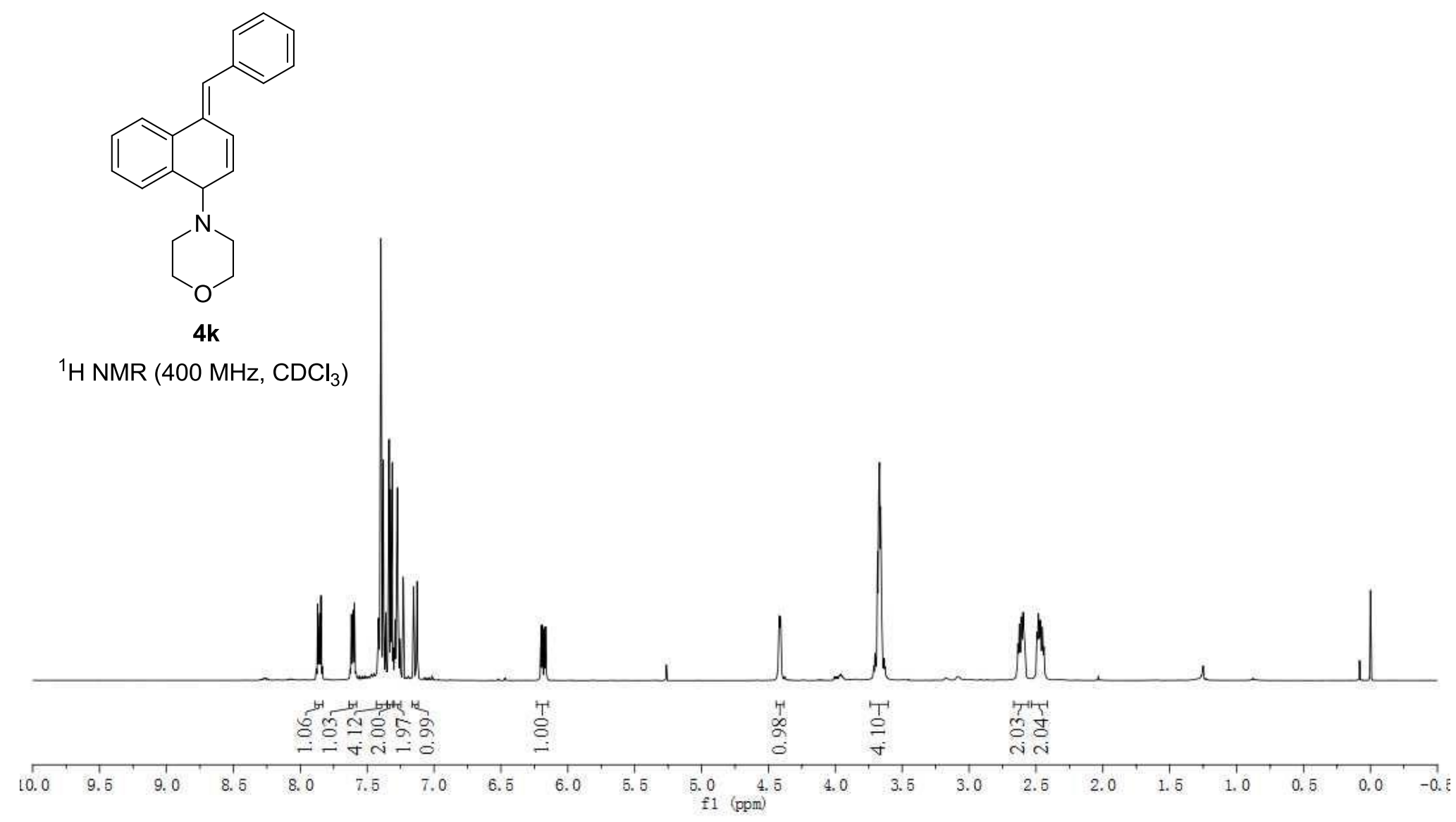

S160 


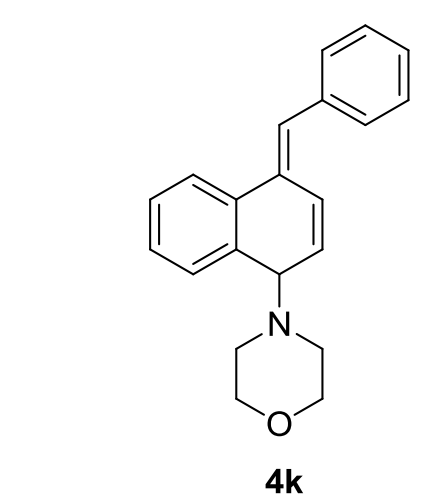

${ }^{13} \mathrm{C}$ NMR $\left(100 \mathrm{MHz}, \mathrm{CDCl}_{3}\right)$

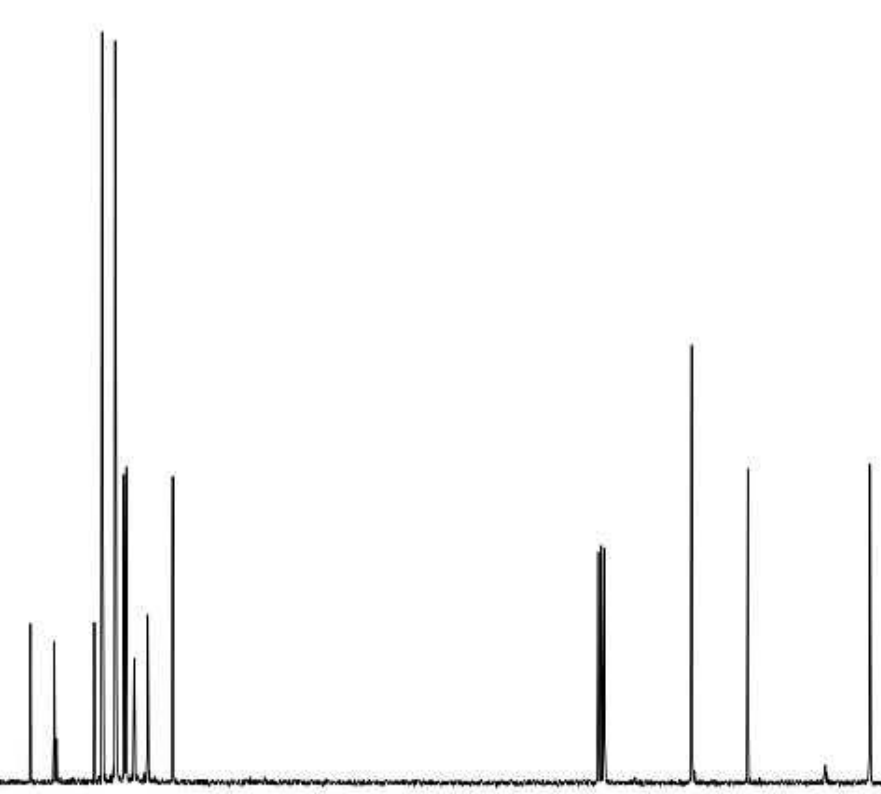

$\begin{array}{lllllllllllll}190 & 180 & 170 & 160 & 150 & 140 & 130 & 120 & 110 & 100 \\ \mathrm{f1}(\mathrm{ppm}) & 90\end{array}$ 


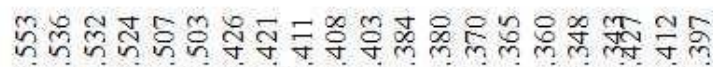

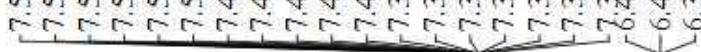

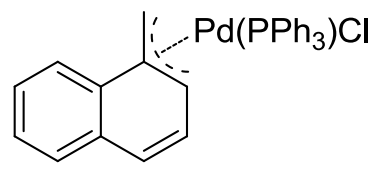

5 aaa

${ }^{1} \mathrm{H}$ NMR $\left(400 \mathrm{MHz}, \mathrm{CDCl}_{3}\right)$

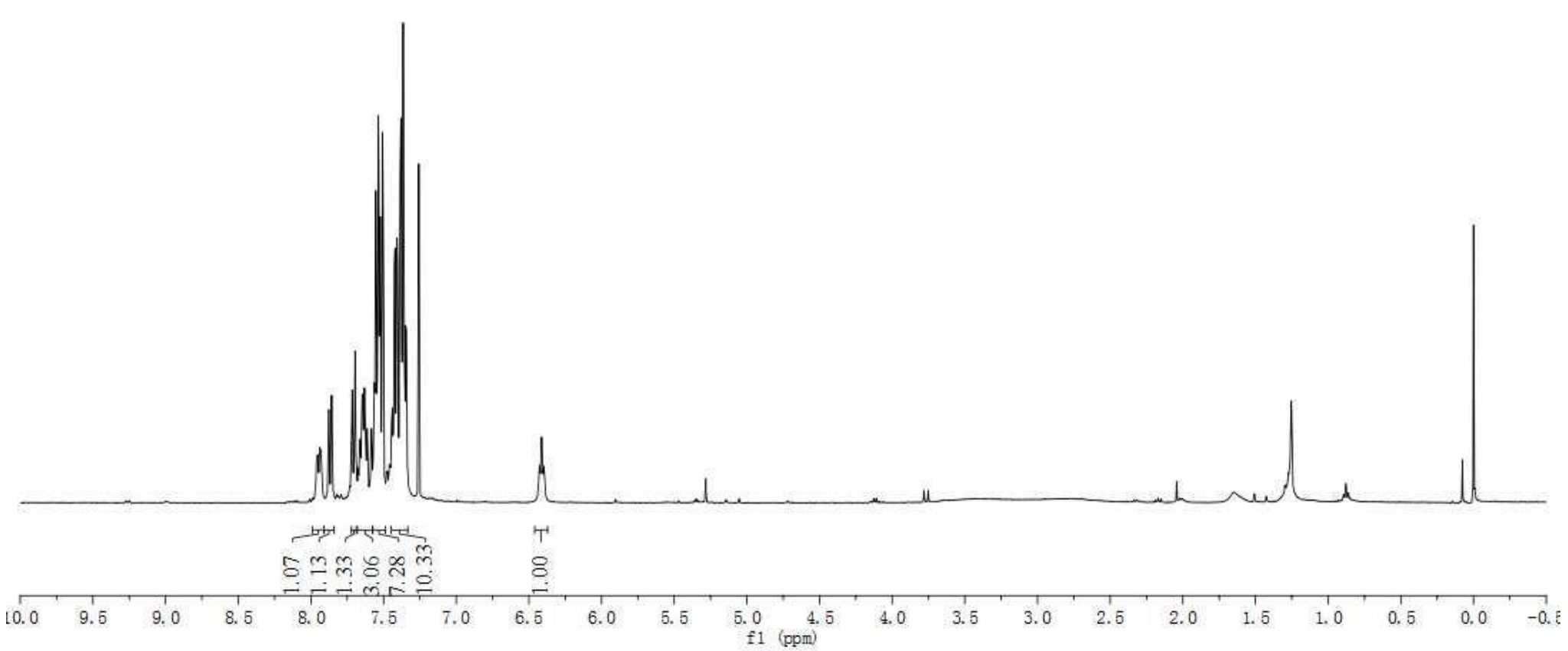

S162 


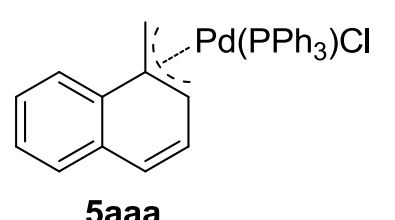

${ }^{13} \mathrm{CNMR}\left(100 \mathrm{MHz}, \mathrm{CDCl}_{3}\right)$

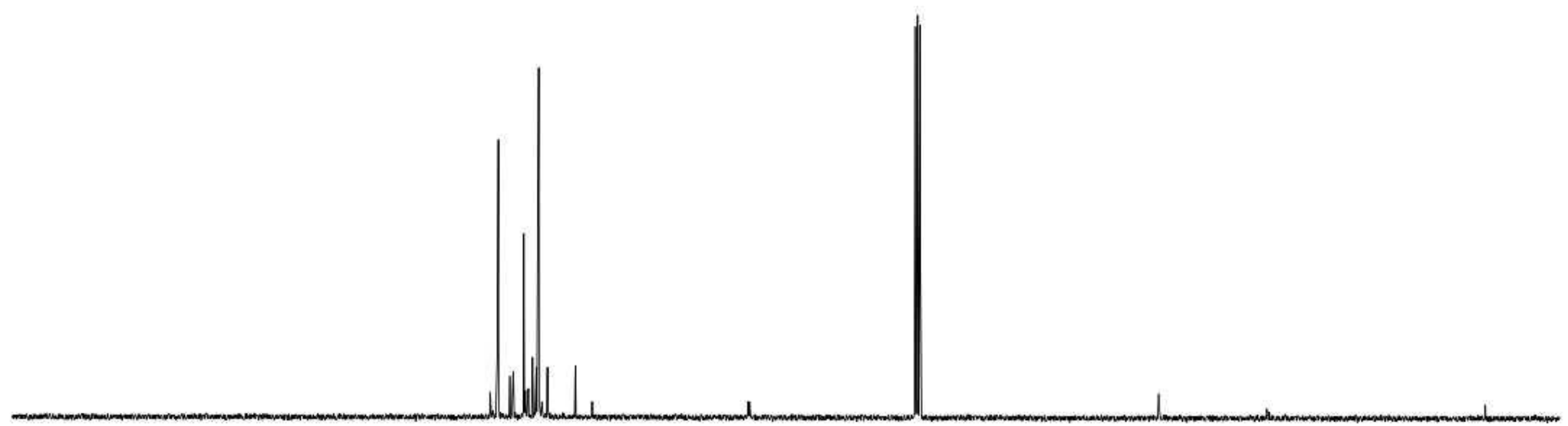

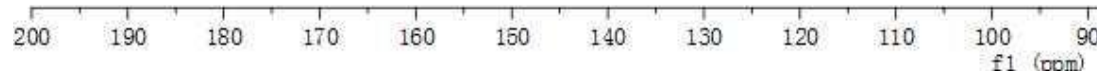




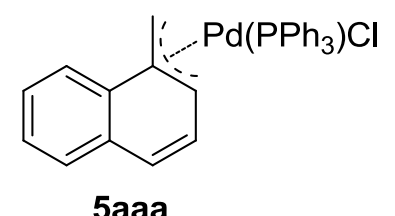

${ }^{31} \mathrm{P}$ NMR $\left(162 \mathrm{MHz}, \mathrm{CDCl}_{3}\right)$

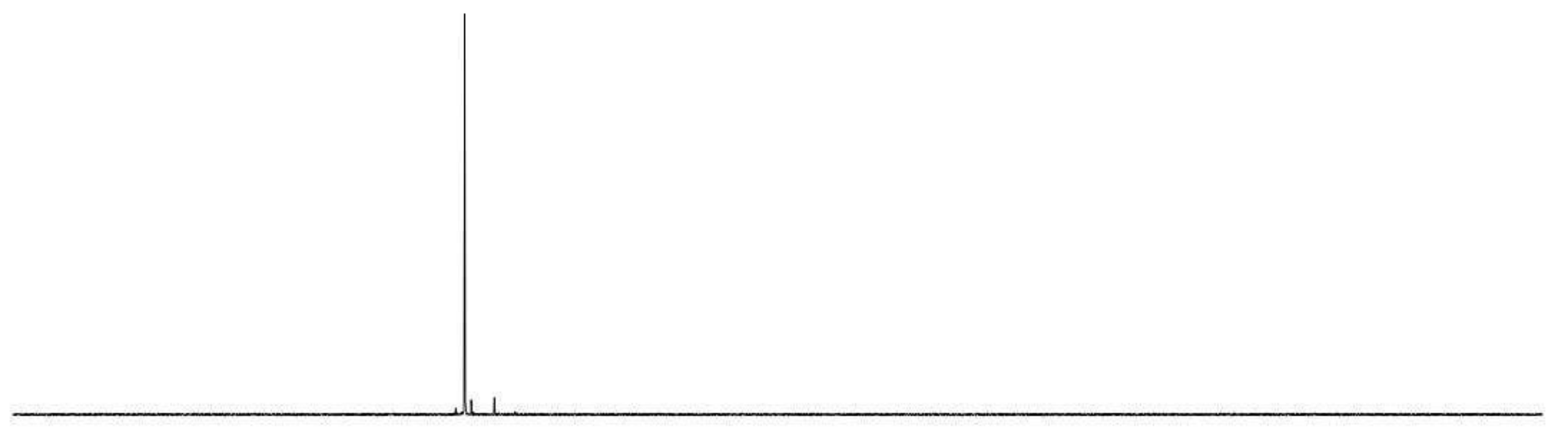

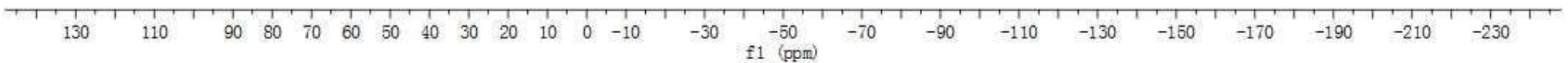




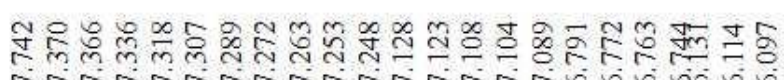

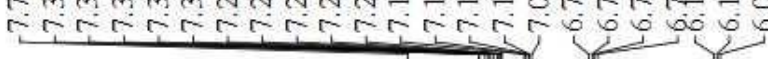

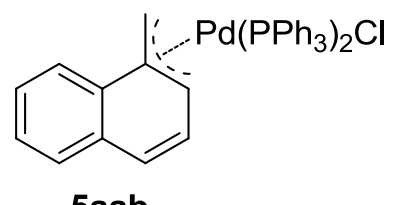

${ }^{1} \mathrm{H} \mathrm{NMR}\left(400 \mathrm{MHz}, \mathrm{CDCl}_{3}\right)$

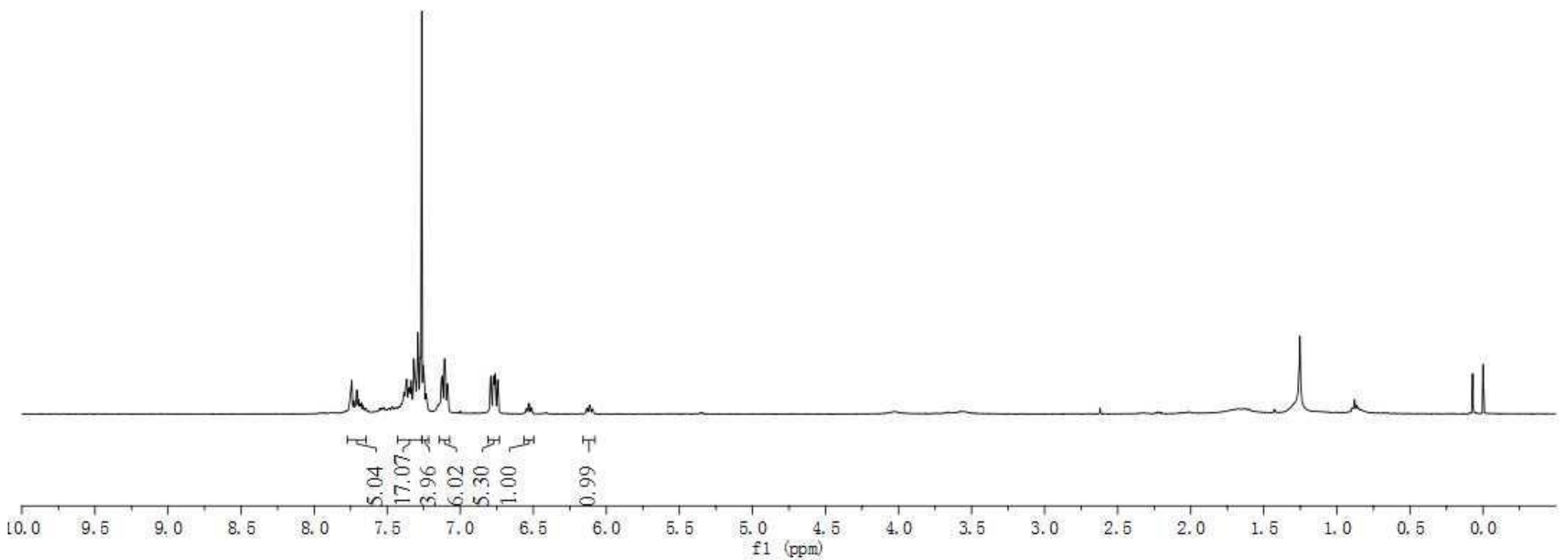




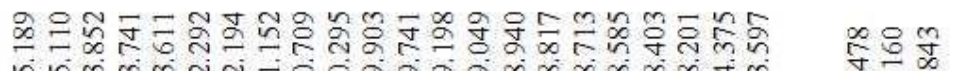

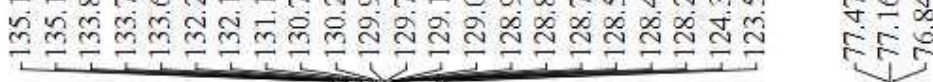

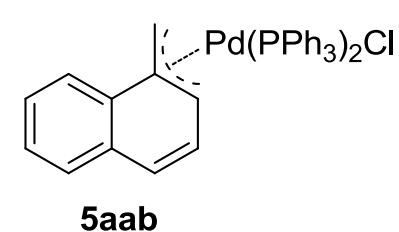

${ }^{13} \mathrm{C}$ NMR $\left(100 \mathrm{MHz}, \mathrm{CDCl}_{3}\right)$

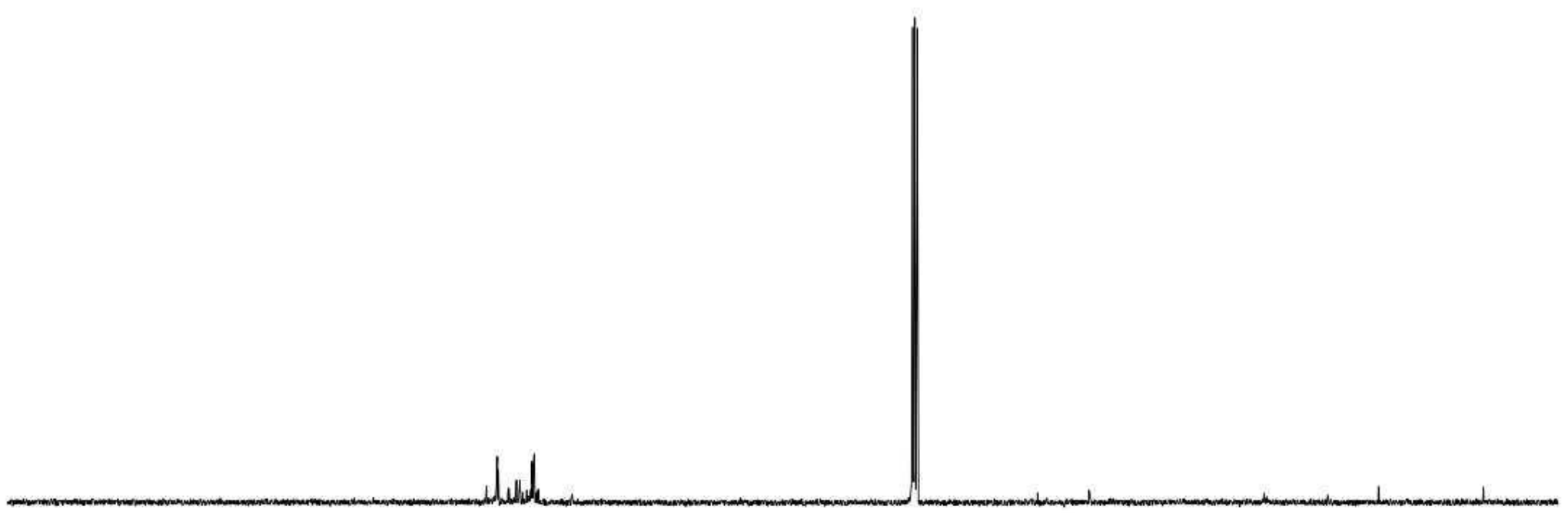

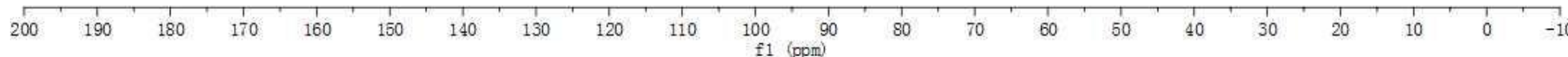

S166 

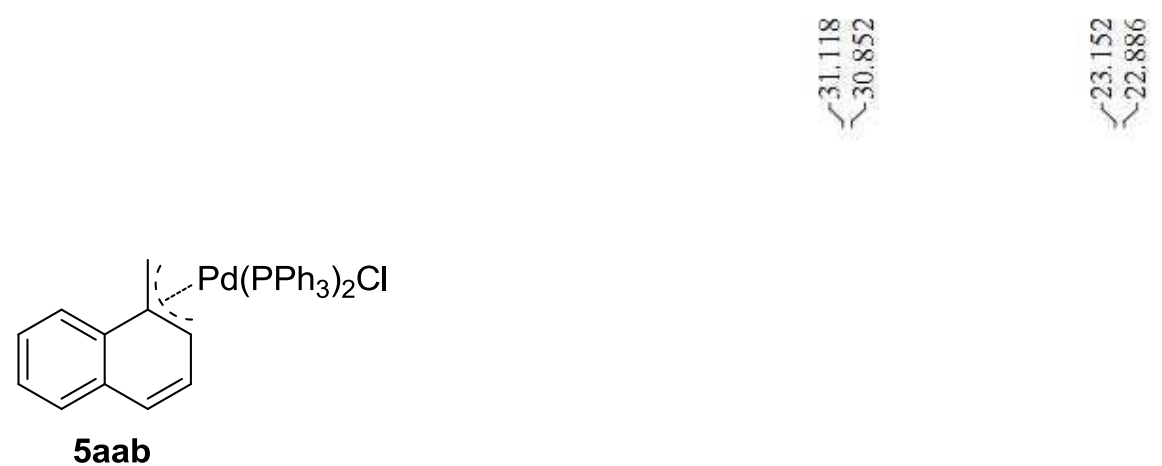

${ }^{31} \mathrm{P}$ NMR $\left(162 \mathrm{MHz}, \mathrm{CDCl}_{3}\right)$

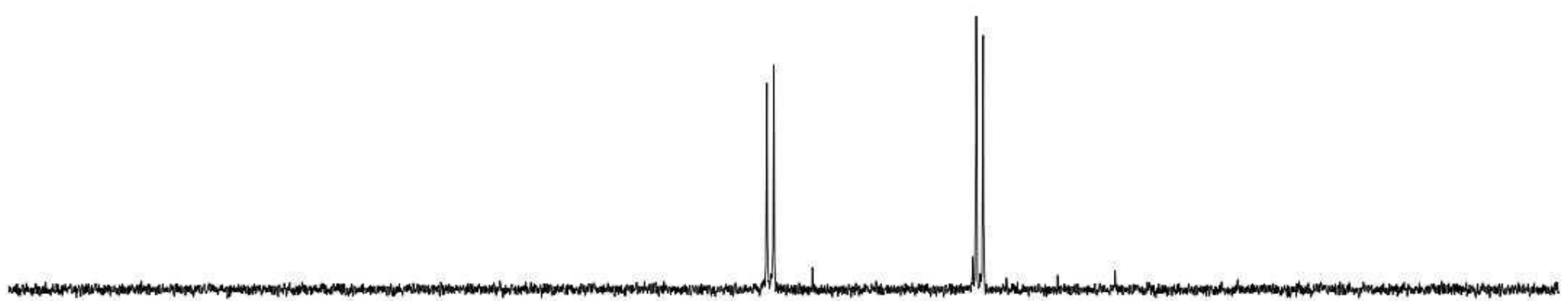

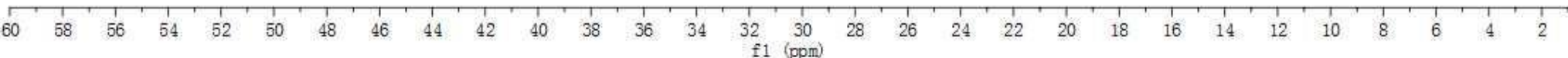

